\title{
Investigation of the Mitochondrial Contact Site and Cristae Organizing System and Its Role in Cristae Formation
}

\author{
Dissertation \\ for the award of the degree \\ „Doctor rerum naturalium” \\ of the Georg-August-Universität Göttingen
}

within the doctoral program

Biology

of the Georg-August University School of Science (GAUSS)

submitted by

Till Stephan

from Celle

Göttingen, 2020 



\section{Members of the Thesis Committee:}

\section{Prof. Dr. Stefan Jakobs}

Clinic of Neurology

University Medical Center, Göttingen and

Research Group Mitochondrial Structure and Dynamics

Max Planck Institute for Biophysical Chemistry, Göttingen

\section{Prof. Dr. Ralf Ficner}

Department of Molecular Structural Biology

Georg-August-University, Göttingen

\section{Members of the Examination Board:}

Prof. Dr. Stefan Jakobs ( $1^{\text {st }}$ Referee $)$

Clinic of Neurology

University Medical Center, Göttingen and

Research Group Mitochondrial Structure and Dynamics

Max Planck Institute for Biophysical Chemistry, Göttingen

Prof. Dr. Ralf Ficner ( $2^{\text {nd }}$ Referee $)$

Department of Molecular Structural Biology

Georg-August-University, Göttingen

Prof. Dr. Dr. h.c. mult. Stefan W. Hell

Department of NanoBiophotonics

Max Planck Institute for Biophysical Chemistry, Göttingen

\section{Prof. Dr. Peter Rehling}

Cellular Biochemistry

University Medical Center, Göttingen

\section{Prof. Dr. Michael Thumm}

Cellular Biochemistry

University Medical Center, Göttingen

\section{PD Dr. Thomas Teichmann}

Department of Plant Cell Biology

Georg-August-University, Göttingen 


\section{Affidavit}

I hereby declare that my dissertation entitled „Investigation of the mitochondrial contact site and cristae organizing system and its role in cristae formation" is my own work that has been written independently and with no other sources and aids than quoted. Where the work was done in collaboration with others, my contributions are clearly indicated throughout the thesis. This thesis, or parts thereof, have not been submitted elsewhere for any academic award or qualification.

Göttingen, April 17, 2020

Till Stephan 


\section{Abstract}

Mitochondria are essential organelles in eukaryotic cells that feature a unique doublemembrane architecture. The mitochondrial inner membrane forms numerous invaginations, named cristae, which can adopt different shapes and sizes. The cristae membrane harbors the protein machinery that facilitates the function of mitochondria as powerhouses of the cell. Small openings, referred to as crista junctions, connect the cristae membrane to the inner boundary membrane, which runs in parallel to the outer membrane. The intricate shape of the inner membrane is determined by a set of membrane-shaping proteins including the dimeric $\mathrm{F}_{1} \mathrm{~F}_{\mathrm{O}}$-ATP synthase, the dynamin-like GTPase optic atrophy 1 (OPA1) and the mitochondrial contact site and cristae organizing system (MICOS), a heterooligomeric protein complex that is crucial for the formation of crista junctions. In mammals, MICOS consists of seven different subunits that are organized within two distinct MICOS subcomplexes. Although several studies demonstrated physical or genetic interactions between the MICOS subcomplexes, OPA1 and the dimeric $\mathrm{F}_{1} \mathrm{~F}_{\mathrm{O}}$-ATP synthase, their precise interplay in cristae formation and maintenance is largely unknown. Consequently, the mechanism of cristae formation is still under debate and a variety of conflicting models describing the formation of cristae have been suggested.

Examination of such models requires the analysis of morphological changes of the inner membrane architecture and the determination of the intramitochondrial localization of the involved membrane-shaping factors. As the diameter of mitochondria is close to the diffraction limit of optical microscopy, visualization of both aspects requires the application of diffractionunlimited fluorescence nanoscopy techniques. However, the lack of adequate labeling strategies for the mitochondrial inner membrane has prohibited the visualization of its dynamics by fluorescence nanoscopy.

The first part of this thesis introduces a reliable labeling approach that enables time-lapse recordings of individual cristae with a resolution of about $50 \mathrm{~nm}$ using stimulated emission depletion (STED) nanoscopy. Live-cell recordings of mitochondria demonstrated that cristae constantly change their appearance on the timescale of seconds and form well-organized groups inside the mitochondrial tubules.

The second part of this thesis investigates the role of the MICOS complex in the organization of cristae and the positioning of crista junctions in mitochondria from Saccharomyces cerevisiae and from different human cell types. STED nanoscopy of Mic60, a core subunit of the MICOS complex, revealed that Mic60 forms spatially coordinated protein clusters, which reflect the distribution of the crista junctions. Frequently, distinct Mic60 clusters are organized in two opposite distribution bands, which run along the mitochondrial tubules. These opposite bands can adopt a helically twisted arrangement, supporting the idea that individual crista junctions are physically coupled along and across the mitochondrial tubules. 3D electron microscopy and STED nanoscopy data indicated that this junction coupling is largely 
independent from the cristae, but is instead an intrinsic feature of the mitochondrial inner membrane.

The central part of this thesis further investigates the mechanisms that control cristae formation in humans. The findings demonstrate that an intricate interplay between the two MICOS subcomplexes, OPA1, and the dimeric $\mathrm{F}_{1} \mathrm{~F}_{\mathrm{O}}$-ATP synthase controls inner membrane remodeling, the formation of cristae, and the coordinated positioning of the crista junctions. HeLa cells were individually depleted of all known MICOS subunits and were analyzed using protein-biochemistry, super-resolution imaging, and electron microscopy. The presented data revealed that the Mic60-subcomplex enables the formation of crista junctions, whereas the Mic10-subcomplex modulates the formation of lamellar cristae. The generation of inducible stable cell lines allowed for the restoration of lamellar cristae upon re-expression of the MICOS complex in MICOS-depleted cells. Reconstitution of the MICOS complex triggered fission of disordered cristae as well as the de novo formation of crista junctions on preexisting cristae. STED recordings further demonstrated that association of the two MICOS subcomplexes, along with the dimeric $\mathrm{F}_{1} \mathrm{~F}_{\mathrm{O}}$-ATP synthase, controls the width of the opposite Mic60 distribution bands and thereby the positioning of crista junctions around the mitochondria. Contradicting previous reports from yeast, the formation of lamellar cristae in humans was found to be largely independent of fusion and fission of mitochondrial tubules as demonstrated by transient depletion of several important fusion and fission factors. Nevertheless, knockdown experiments illustrated that the fusion protein OPA1 stabilizes tubular crista junctions and controls the formation of Mic60 assemblies together with Mic10. Therefore, OPA1, together with the dimeric $\mathrm{F}_{1} \mathrm{~F}_{\mathrm{O}}$-ATP synthase, influences the positioning of the MICOS complex in the inner membrane.

Finally, the findings described in this work allowed for the development of a new model of cristae formation in which the interplay of the MICOS-subcomplexes with OPA1 and with the dimeric $\mathrm{F}_{1} \mathrm{~F}_{\mathrm{O}}$-ATP synthase controls the remodeling of the inner membrane and facilitates the segmentation of unstructured cristae membranes into multiple highly organized lamellar cristae. 


\section{Preface}

This doctoral thesis summarizes my work on the structure and dynamics of the mitochondrial inner membrane and the factors that are necessary for the formation and maintenance of mitochondrial cristae. The underlying experimental work resulted in the following manuscripts and publications:

\section{Research articles included in this thesis:}

Till Stephan, Axel Rösch, Dietmar Riedel, Stefan Jakobs (2019): "Live-cell STED nanoscopy of mitochondrial cristae"

Scientific Reports 9, 12419. DOI: 10.1038/s41598-019-48838-2

Stefan Stoldt, Till Stephan ${ }^{*}$, Daniel C. Jans, Christian Brüser, Felix Lange, Jan KellerFindeisen, Dietmar Riedel, Stefan W. Hell and Stefan Jakobs (2019): "Mic60 exhibits a coordinated clustered distribution along and across yeast and mammalian mitochondria"

PNAS 116 (20), 9853-9858. DOI: 10.1073/pnas.1820364116

Till Stephan ${ }^{*}$, Christian Brüser*, Markus Deckers, Anna M. Steyer, Francisco Balzarotti, Mariam Barbot, Tiana S. Behr, Gudrun Heim, Wolfgang Hübner, Peter Ilgen, Felix Lange, David Pacheu-Grau, Jasmin Pape, Stefan Stoldt, Thomas Huser, Stefan W. Hell, Wiebke Möbius, Peter Rehling, Dietmar Riedel and Stefan Jakobs (2020): "MICOS assembly controls mitochondrial inner membrane remodeling and crista junction redistribution to mediate cristae formation"

The EMBO Journal (revised manuscript under peer review).

*Equal contributions

\section{Other publications related to this thesis:}

Raffaele Faoro, Margherita Bassu, Yara X. Mejia, Till Stephan, Nikunj Dudani, Christian Boeker, Stefan Jakobs and Thomas P. Burg (2018): “Aberration-corrected cryoimmersion light microscopy"

PNAS 115 (6), 1204 - 1209. DOI: 10.1073/pnas.1717282115

Stefan Jakobs, Till Stephan, Peter Ilgen and Christian Brüser (2020): "Light Microscopy of Mitochondria at the Nanoscale"

Annual Review of Biophysics 49:291-310. DOI: 10.1146/annurev-biophys-121219-081550 


\section{Table of Contents}

Abstract.

Preface

III

Table of Contents.

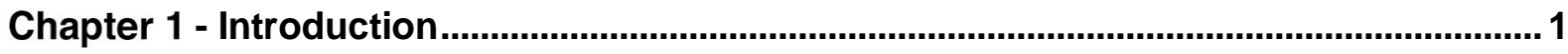

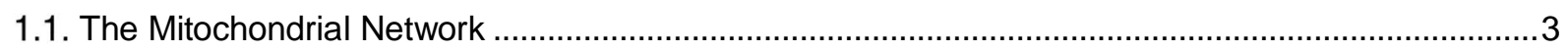

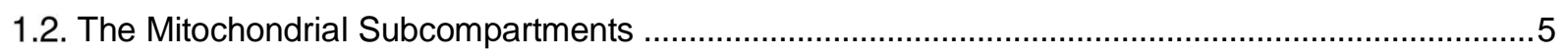

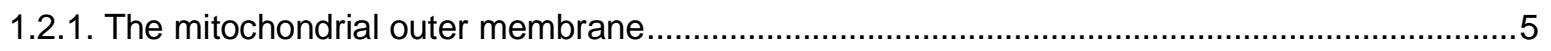

1.2.2. The matrix

1.2.3. The mitochondrial inner membrane .............................................................................. 7

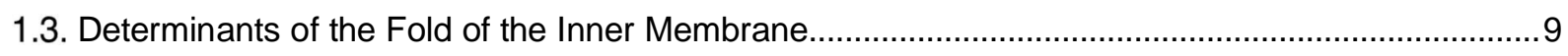

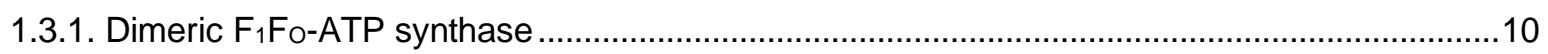

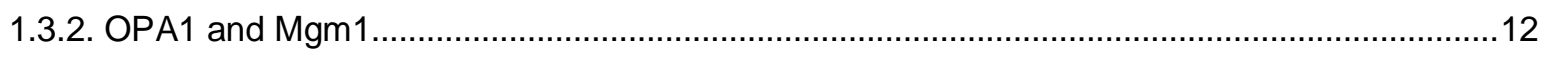

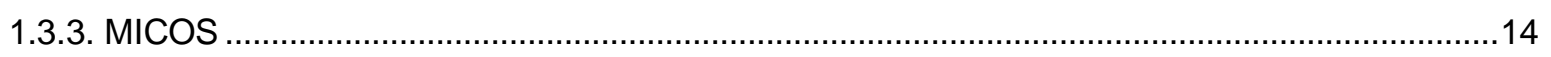

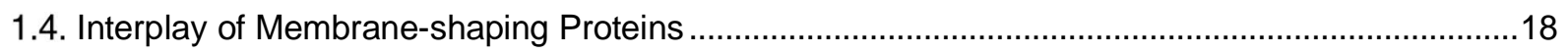

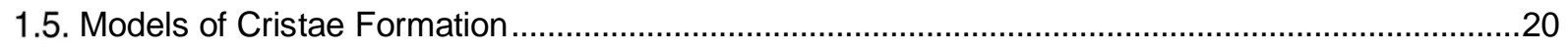

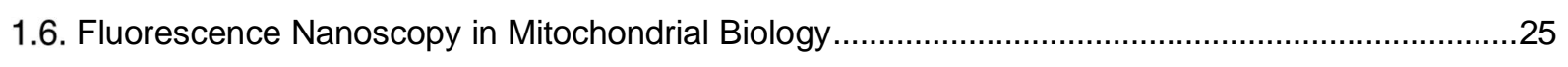

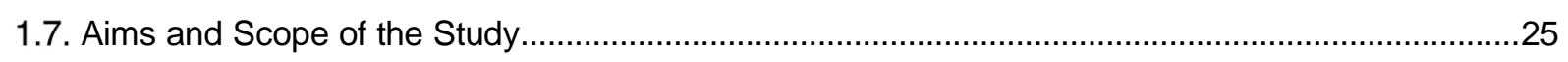

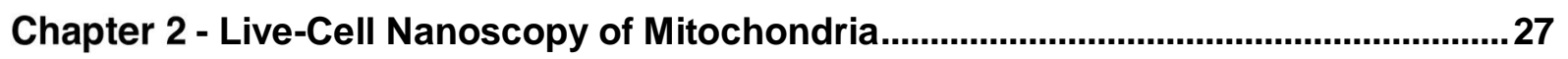

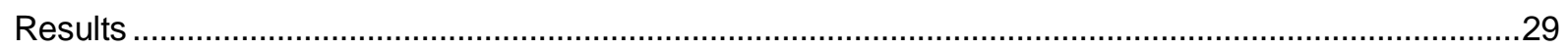

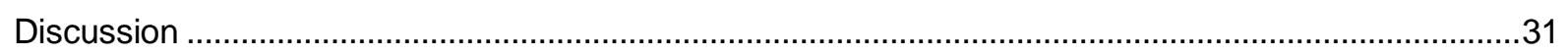

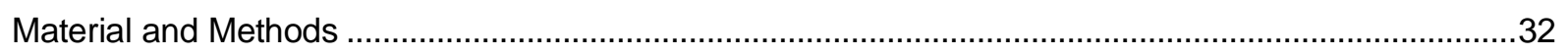

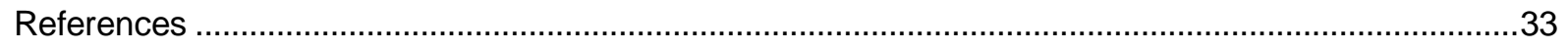

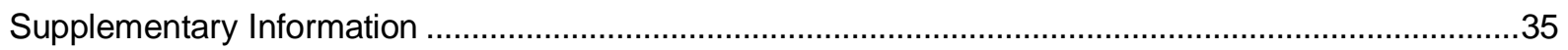

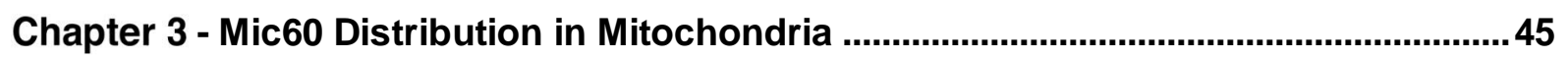

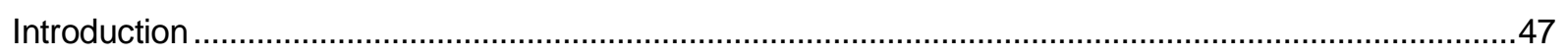

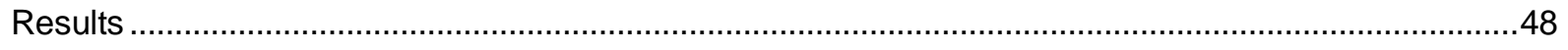

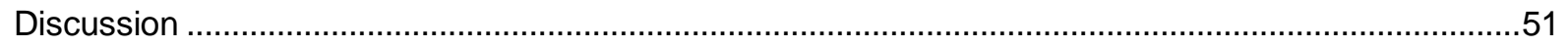

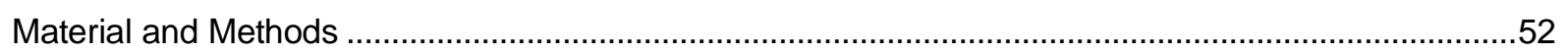

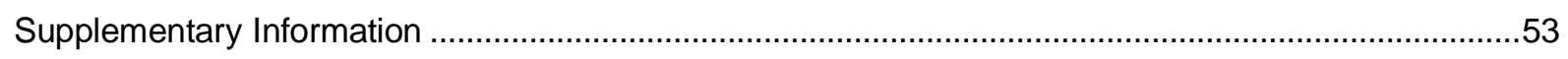


Chapter 4 - Role of MICOS in Cristae Formation .............................................................. 75

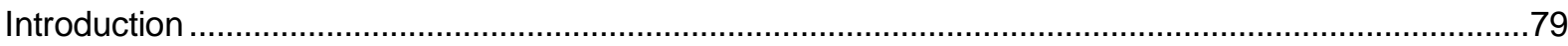

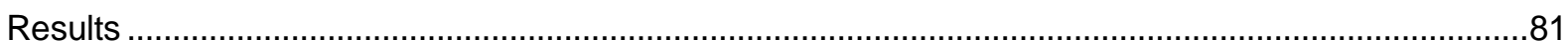

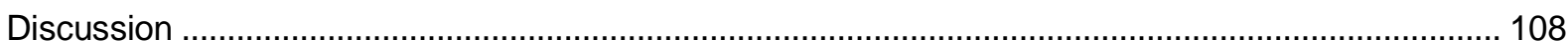

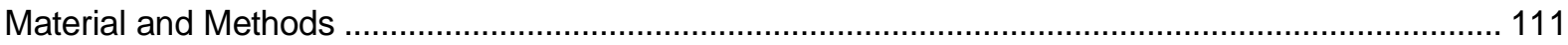

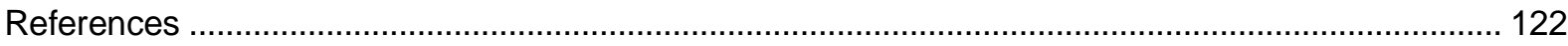

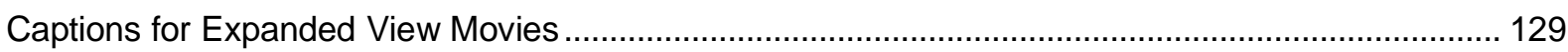

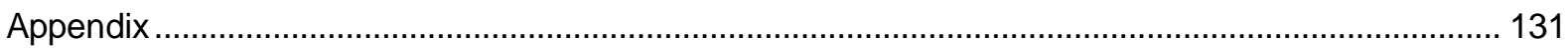

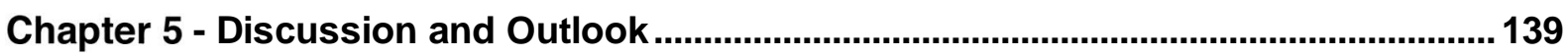

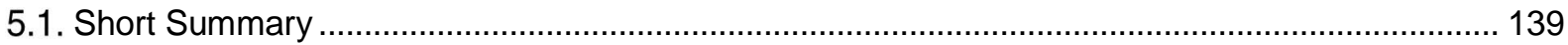

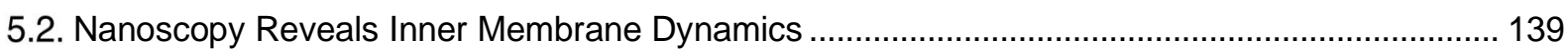

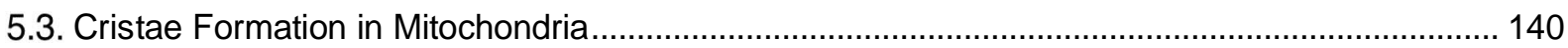

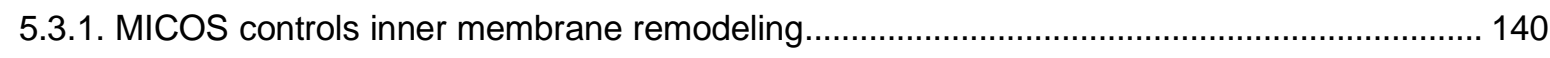

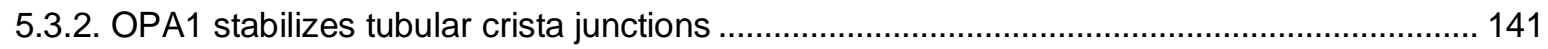

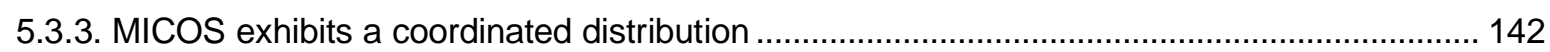

5.3.4. Mic10 regulates the width of the Mic60 distribution bands ............................................... 143

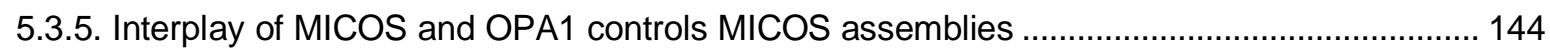

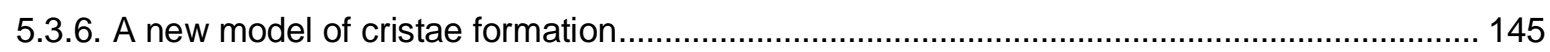

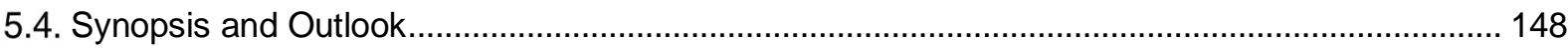

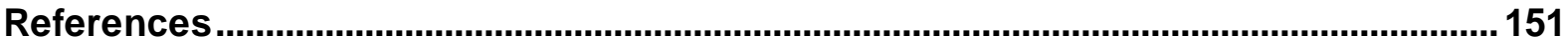

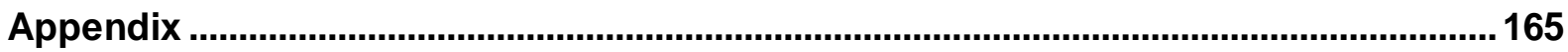

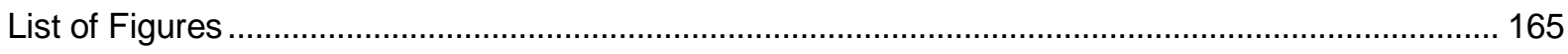

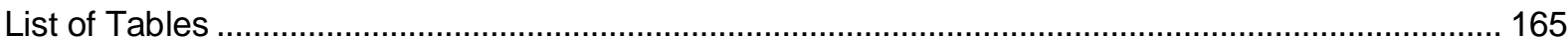

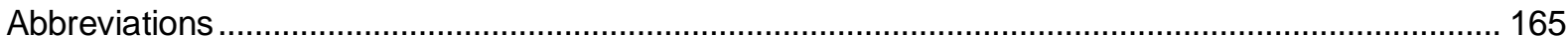

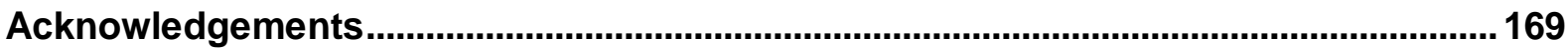




\section{Chapter 1 - Introduction}

Mitochondria were first observed by Albert von Kölliker, who described "granules" that were arranged in the sarcoplasm of striated muscle tissue (Kölliker, 1856). Later, Richard Altmann visualized similar structures also inside other cell types and considered them to be organisms with genetic and metabolic autonomy living inside larger cells (Altmann, 1890). In 1898, Carl Benda finally termed these structures, with regard to their heterogeneous appearance, as mitos (Greek, threads) and chondros (Greek, granules) (Benda, 1898).

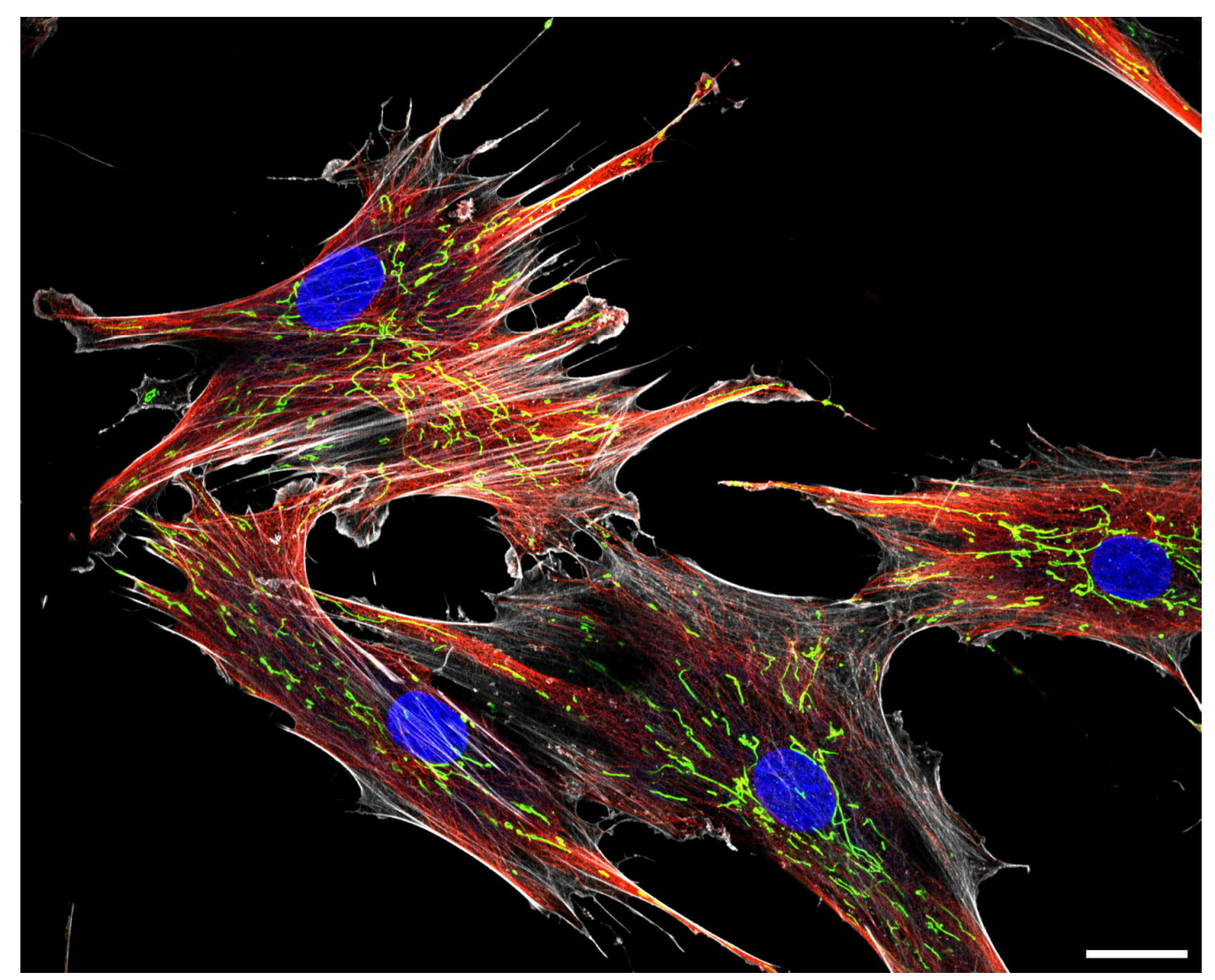

Figure 1: Mitochondria form networks. Human dermal fibroblasts (HDFa) labeled for mitochondria (green), nuclear DNA (blue), tubulin filaments (red) and actin filaments (gray). The mitochondria form extended networks of tubules and granules that pervade the cell. Scale bar: $20 \mu \mathrm{m}$.

Today, we know that mitochondria are essential eukaryotic organelles. The endosymbiotic theory proposes that they evolved from a prokaryote which was engulfed by the progenitor of today's eukaryotic cells (Mereschkowsky, 1910; Sagan, 1967). Mitochondria fulfil a plethora of different functions in eukaryotic cells. Most importantly, they are often referred to as the "powerhouses of the cell", as they generate the majority of adenosine triphosphate (ATP) by oxidative phosphorylation (OXPHOS). They are also pivotal for other pathways and signaling cascades within the cell, including fatty acid oxidation, the citric acid cycle, heme biosynthesis, amino acid biosynthesis, the synthesis of iron-sulfur clusters, and the induction of apoptosis 
(Giacomello et al., 2020; Scheffler, 2007; Wallace, 2005). The functional versatility of mitochondria requires a complex interconnection with many other cellular components. Therefore, mitochondria usually form extended dynamic networks of tubular structures that pervade the cell (Figure 1) and that form contact sites with other organelles. Due to their endosymbiotic origin, mitochondria feature a unique double-membrane architecture (Palade, 1952; Sjöstrand, 1953). The smooth outer membrane separates the mitochondria from the cytosol. The inner membrane exhibits a much larger surface area than the outer membrane and features numerous invaginations called cristae, which point inwards towards the matrix of the organelle (Figure 2). These cristae divide the inner membrane into different functional domains: the inner boundary membrane, which runs parallel to the outer membrane, the cristae membrane, and crista junctions, which are small confined openings that connect the cristae membrane with the inner boundary membrane.
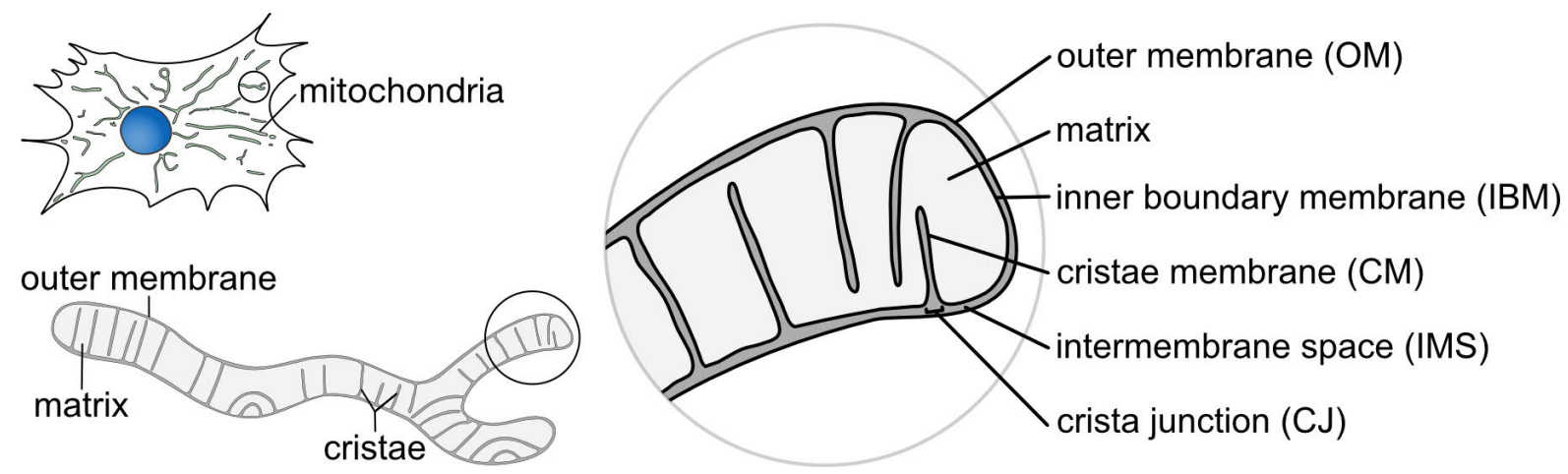

Figure 2: The double-membrane architecture of mitochondria. Most eukaryotic cells contain an extended network of mitochondrial tubules. Mitochondria feature a smooth outer membrane and a highly convoluted inner membrane with numerous invaginations, termed cristae. The cristae point into the interior of the organelle (matrix). The inner membrane can be subdivided into the inner boundary membrane (IBM) and the cristae membrane (CM). Crista junctions (CJs) connect both domains of the inner membrane. The mitochondrial outer and inner membrane are separated by an intermembrane space (IMS).

As another remnant of their proteobacterial origin, mitochondria feature a small separate genome of mitochondrial deoxyribonucleic acid (mtDNA) (Gray et al., 1999; Nass and Nass, 1963). The human mtDNA is a circular molecule with a size of about 16.6 kilo base pairs that contains 37 genes encoding 13 proteins, two ribosomal ribonucleic acids (rRNAs) and 22 different transfer RNAs (Anderson et al., 1981). Packed into nucleoproteins, the mtDNA forms mitochondrial nucleoids. Up to thousands of these nucleoids can be distributed along the mitochondrial tubules in a single cell (Alam et al., 2003).

Due to the organelle's dynamics and sub-compartmentalization, mitochondrial processes take place across very different spatial scales. They can affect the entire mitochondrial network, single mitochondria, or even individual cristae. The following section will highlight the dynamics of the mitochondrial network. 


\subsection{The Mitochondrial Network}

In most cell types mitochondria form extended networks of tubules which constantly undergo fusion and fission (Bereiter-Hahn and Vöth, 1994). Motor proteins facilitate an active transport of mitochondria along the tubulin and actin cytoskeleton throughout the cell (Fehrenbacher et al., 2004; Morris and Hollenbeck, 1995), which allows the mitochondrial network to adapt dynamically to different physiological conditions (Detmer and Chan, 2007; Giacomello et al., 2020). Since mitochondrial fusion and fission are essential for a constant mixture of mitochondrial proteins, lipids and the exchange of the nucleoids, dysfunction of the mitochondrial fusion and fission machinery disturbs lipid homeostasis and can lead to a loss of the mtDNA (Jones and Fangman, 1992; Kojima et al., 2019; Westermann, 2010). As a consequence, several severe human diseases like Charcot-Marie-Tooth disease, optic atrophy, and Behr syndrome are associated with disturbed mitochondrial fusion-fission dynamics (Alexander et al., 2000; Baxter et al., 2001; Giacomello et al., 2020). Under healthy conditions, the mitochondrial network morphology is tightly controlled by several mitochondrial fusion and fission factors, which are found on mitochondria but also the endoplasmic reticulum (ER).

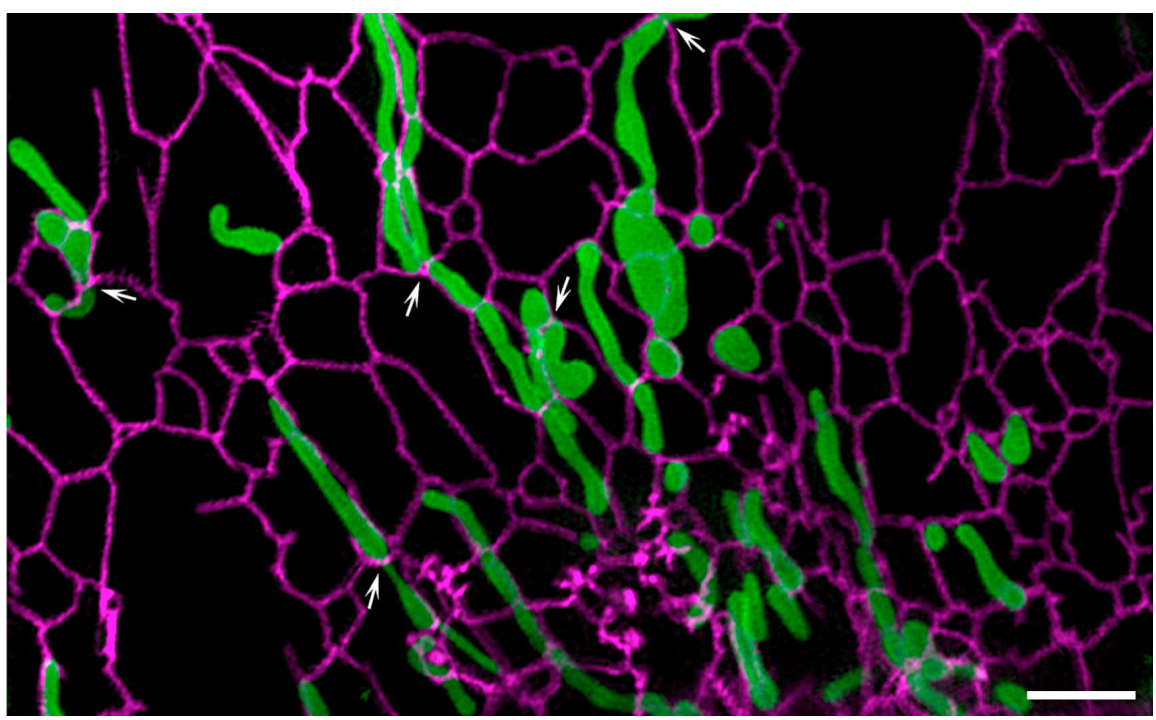

Figure 3: Mitochondria and endoplasmic reticulum. Live-cell recording of mitochondria (green) and endoplasmic reticulum (ER, magenta) in COS7 (CV-1 in origin, carrying SV40) cells. The arrows mark contact sites of mitochondria and the ER. Scale bar: $3 \mu \mathrm{m}$. Adapted with permission from: Guo et al., 2018.

Mitochondria undergo frequent interactions with the ER to facilitate lipid exchange or transmission of calcium signals (Friedman and Nunnari, 2014; Friedman et al., 2018; Giacomello et al., 2020; Rusiñol et al., 1994). Live-cell fluorescence microscopy has demonstrated that both fusion and fission of mitochondrial tubules occur preferentially at ERmitochondria contact sites (Figure 3), as the protein machineries involved in both processes are enriched at such sites (Abrisch et al., 2020; Friedman et al., 2011; Guo et al., 2018; Korobova et al., 2013). 
The role of the ER is well understood in mitochondrial fission. Prior to fission, actin polymerization pre-constricts the mitochondrial tubules at the ER-mitochondria interface and the dynamin-related protein 1 (DPR1; Dnm1 in yeast), a GTPase essential for fission, is recruited from the cytosol to mitochondria (Figure 4). DRP1 oligomerizes, forming large helical assemblies that further constrict the mitochondrial tubules and complete fission (Chakrabarti et al., 2017; Korobova et al., 2013; Manor et al., 2015).
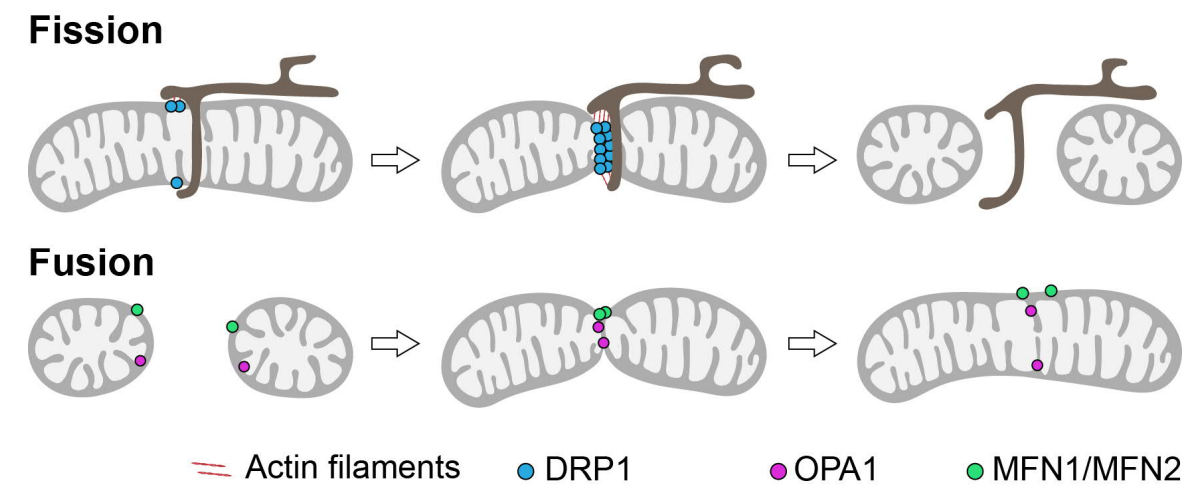

Figure 4: Fusion and fission of mitochondria. Fission of mitochondrial tubules occurs at ERmitochondria contact sites. Following pre-constriction by actin filaments, DRP1 assembles into a helical filament that facilitates the fission of the mitochondrial tubule. Fusion of the mitochondrial outer membrane is mediated by the mitofusins MFN1 and MFN2. OPA1 is required for fusion of the mitochondrial inner membrane.

Outer membrane fusion depends on the GTPases mitofusin 1 (MFN1; in yeast fuzzy onions homolog 1, Fzo1) and mitofusin 2 (MFN2) (Eura et al., 2003; Hermann et al., 1998; Legros et al., 2002; Rapaport et al., 1998; Santel and Fuller, 2001). MFN1 dimerizes in a GTP-dependent manner and thereby controls the membrane contact of two mitochondria (Qi et al., 2016). Despite its high homology to MFN1, different functions were suggested for MFN2 (Ishihara et al., 2004; Rojo et al., 2002). Several studies have suggested that MFN2 functions as a tether between mitochondria and the ER (Brito and Scorrano, 2008; Sugiura et al., 2013). However, also conflicting results have been reported (Filadi et al., 2015) and thus, the precise function of MFN2 is still a highly debated issue (Filadi et al., 2015; Naon et al., 2017).

Following outer membrane fusion, the inner membranes of previously separated mitochondria must also be fused together. The dynamin-like protein optic atrophy 1 (OPA1; in yeast mitochondrial genome maintenance 1, Mgm1) works in concert with MFN1 and is the main factor involved in inner membrane fusion (Lee et al., 2004; Legros et al., 2002; Meeusen et al., 2006; Sesaki et al., 2003). Additionally, OPA1 influences the fold of the inner membrane and controls the diameter of crista junctions in apoptosis (Frezza et al., 2006; Griparic et al., 2004; Meeusen et al., 2006). Therefore, it is considered to be one of the key players that facilitate the formation of cristae in mitochondria (Giacomello et al., 2020). Chapter 1.3.2 further discusses the role of OPA1/Mgm1 in inner membrane shaping. 


\subsection{The Mitochondrial Subcompartments}

The double-membrane architecture of mitochondria allows for a subcompartmentalization of the organelle (Figure 5). Each of the mitochondrial subcompartments serves different purposes. The following section provides a short overview over the different functions of both the mitochondrial inner and outer membrane and also of the matrix of the organelle.

A

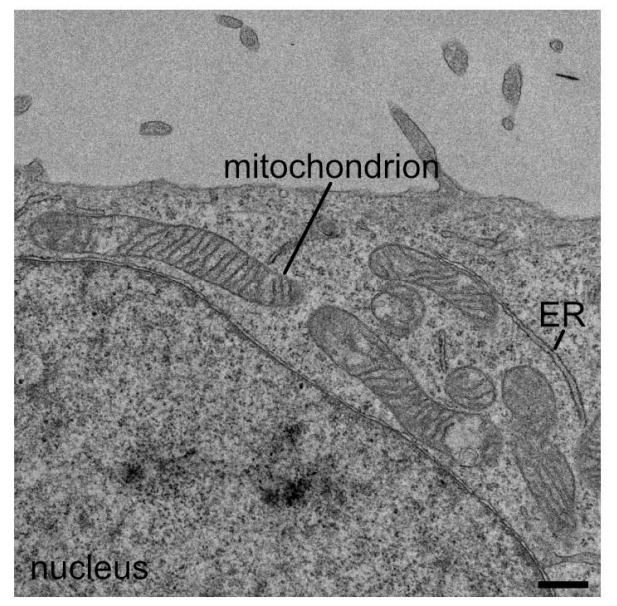

B

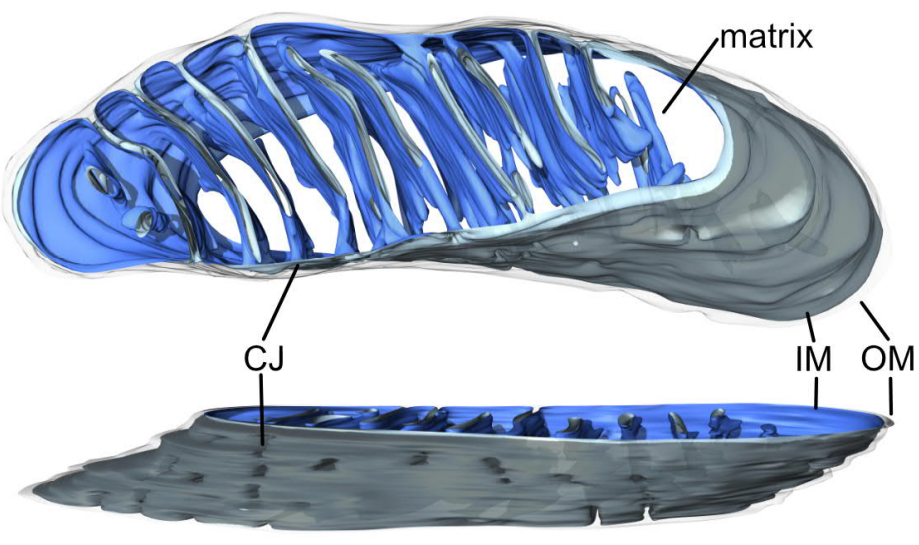

Figure 5: Mitochondrial subcompartmentalization. (A) Transmission electron microscopy (TEM) recording of a HeLa cell shows mitochondria next to the nucleus and ER tubules. (B) Reconstruction of an electron tomography recording of a mitochondrion from a HeLa cell. The outer membrane (OM) is shown in transparent grey and the inner membrane (IM) in blue. The side of the inner membrane that faces the matrix is shown in dark blue and the side that faces the intermembrane space is shown in light blue. A side view (lower panel) provides a view onto the crista junctions (CJs) through the transparent outer membrane. Scale bars: $500 \mathrm{~nm}$.

\subsubsection{The mitochondrial outer membrane}

\subsubsection{The outer membrane is an interaction and signaling platform}

The mitochondrial outer membrane separates the mitochondria from the cytosol and represents the interface for interactions of mitochondria with other cellular organelles and structures like the cytoskeleton. The active transport of mitochondria along microtubules, for example, is realized by the binding of kinesin- 1 to the outer membrane protein mitochondrial Rho 1 (MIRO1) via the adapters trafficking kinesin-binding protein 1 and 2 (TRAK1 and TRAK2) (Brickley and Stephenson, 2011; Glater et al., 2006). Similarly, myosin XIX can interact with MIRO1 and MIRO2 and thus links the outer membrane to actin filaments (Oeding et al., 2018; Quintero et al., 2009). As mentioned before, interactions of mitochondria with the actin cytoskeleton support mitochondrial fission at ER-mitochondria contact sites. To this end, the outer membrane harbors the actin-nucleating protein Spire1C, which activates the ER-bound actin polymerase inverted formin 2 (INF2) to promote the actin-based constriction of mitochondrial tubules prior to fission (Chakrabarti et al., 2017; Ji et al., 2015; Korobova et al., 
2013; Yang and Svitkina, 2019). Besides interactions with the cytoskeleton, interactions of mitochondria were observed with other cellular organelles. Whereas MFN2 presumably mediates contacts between the ER and mitochondria in mammals (Brito and Scorrano, 2008), the yeast mitofusin Fzo1 serves, for example, as a tether between mitochondria and peroxisomes (Shai et al., 2018).

The outer membrane is also a part of extended signaling cascades, such as in apoptosis. Upon induction of apoptosis, the pro-apoptotic Bcl-2-associated X protein (BAX) can translocate from the cytosol to the mitochondrial outer membrane. There it oligomerizes and facilitates the formation of outer membrane pores (Große et al., 2016; Kalkavan and Green, 2017; SalvadorGallego et al., 2016). Rupture of the outer membrane allows for the release of pro-apoptotic factors like cytochrome $\mathrm{c}$ from the intermembrane space into the cytosol, leading to the activation of caspases and finally cell death. Recent studies further suggest that the Bax-induced pores in the mitochondrial outer membrane also allow the herniation of the mitochondrial inner membrane into the cytosol. Rupture of the inner membrane ultimately releases mtDNA, triggering an inflammatory response by the cGAS-STING (cyclic GMP-AMP synthasestimulator of interferon genes) pathway (McArthur et al., 2018; Riley et al., 2018).

\subsubsection{The outer membrane controls import of proteins and metabolites}

As the nuclear DNA encodes nearly all mitochondrial proteins, mitochondria must import the majority of their proteins from the cytosol and must target them to different sub-mitochondrial compartments to fulfill their functions. The mitochondrial outer membrane harbors the machinery that facilitates the efficient trafficking of precursor proteins into the organelle. The translocase of the outer membrane (TOM complex) is the major entry gate for precursor proteins (Bolliger et al., 1995; Moczko et al., 1997; Wiedemann and Pfanner, 2017). The core of the TOM complex is formed by TOM40, a beta-barrel protein that forms a pore (Hill et al., 1998; Mokranjac and Neupert, 2015; Shiota et al., 2015). Proteins that are destined for the matrix space, the intermembrane space or the inner membrane are passed from the TOM complex to different import machineries. The majority of matrix proteins and many inner membrane proteins are imported by the pre-sequence translocase of the inner membrane (TIM23), whereas multispanning hydrophobic carrier proteins of the inner membrane are usually handed over to the carrier translocase of the inner membrane (TIM22). Many proteins that are destined for the intermembrane space, however, are imported using the mitochondrial import and assembly machinery (MIA) (Callegari et al., 2020; Chacinska et al., 2004; Kiebler et al., 1990; Sirrenberg et al., 1996; Wiedemann and Pfanner, 2017). The TOM complex also imports precursors of $\beta$-barrel proteins that are destined for the outer membrane. These precursors are inserted into the outer membrane by the sorting and assembly machinery (SAM) (Klein et al., 2012; Paschen et al., 2003; Wiedemann et al., 2003). In addition to its role in protein import, the SAM complex fulfils other important functions in mitochondria, as well. It is connected to the endoplasmic reticulum-mitochondria encounter structure (ERMES) that stably connects the ER and mitochondria in yeast (Horvath et al., 2015; Kornmann et al., 2009; 
Yamano et al., 2010). Moreover, the SAM complex is involved in the formation of contact sites between the mitochondrial inner and outer membranes, which are further discussed in chapter 1.3.3.1.

The metabolic processes occurring in mitochondria require a constant exchange of molecules, including ions and metabolites. The best-characterized gate for these molecules is the voltagedependent anion channel (VDAC, often called mitochondrial porin) which is the most abundant outer membrane protein in mitochondria. In mammals, it exists in 3 different isoforms and facilitates, amongst other things, the import of ADP and the export of ATP (Benz, 1994; Mannella, 1992).

\subsubsection{The matrix}

The matrix is the innermost compartment of mitochondria which contains about two thirds of all mitochondrial proteins (Alberts et al., 2017). It fulfills a variety of functions, as it contains the enzymes for many critical metabolic pathways. These include the $\beta$-oxidation of fatty acids, the citric acid cycle, transamination, and parts of the urea cycle (Berg et al., 2017; Eaton et al., 1996; Scheffler, 2007). The citric acid cycle, or tricarboxylic acid (TCA) cycle, facilitates the oxidation of acetyl coenzyme A (Acetyl-CoA) to generate the reducing agents nicotinamide adenine dinucleotide (NADH) and flavin adenine dinucleotide $\left(\mathrm{FADH}_{2}\right)$ which are used by the OXPHOS machinery to regenerate ATP from ADP and inorganic phosphate. At the same time, the TCA cycle also produces important precursors for many nonessential amino acids (Scheffler, 2007).

The matrix also contains the mtDNA and complex machinery that is necessary for its replication, transcription, and for the translation of mtDNA-encoded proteins. This includes, amongst others, a mitochondrial DNA polymerase, a mitochondrial RNA polymerase and mitochondrial ribosomes (Fox, 2012; Holt and Reyes, 2012).

\subsubsection{The mitochondrial inner membrane}

Compared with the mitochondrial outer membrane, the mitochondrial inner membrane features a unique lipid composition, as it is strongly enriched with negatively charged lipids. It exhibits 2.5 times more cardiolipin and 1.5 times more phosphatidylinositol but has reduced levels of phosphatidylcholine and phosphatidylethanolamine (Ardail et al., 1990; Simbeni et al., 1991). Cardiolipin is a vital component of the inner membrane that supports its unique architecture (see also chapter 1.3) and the metabolic function of mitochondria (Fry and Green, 1981). The inner membrane is also highly enriched with proteins, including the OXPHOS complexes and a high number of carrier proteins that control the transport of metabolites across the membrane (Arco and Satrústegui, 2005; Giacomello et al., 2020; Moualij et al., 1997).

It is widely accepted that cristae, the invaginations of the inner membrane, can adopt different shapes for several reasons, such as transitions in the metabolic state of the cell (Cogliati et al., 2016; Hackenbrock, 1966). Moreover, the size and shape of the cristae (Figure 6) can differ 
substantially between different organisms and even between different cell types within the same organism (Munn, 1974; Zick et al., 2008). Cristae shapes range from small tubules in mitochondria from the ciliate Paramecium tetraurelia (Mühleip et al., 2016) to large lamellae in mitochondria from chick cerebellum (Frey et al., 2002).
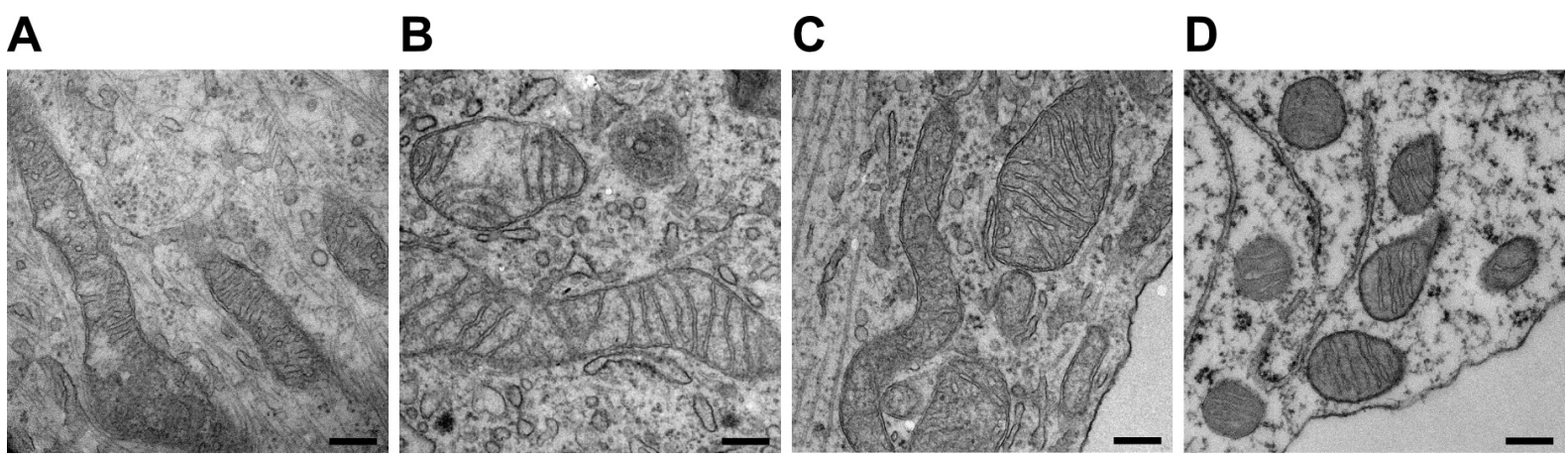

Figure 6: Sizes and shapes of cristae are cell type dependent. Transmission electron microscopy recordings of different cell types. (A) Mitochondria from human dermal fibroblasts. (B) Mitochondria from human HeLa cells. (C) Mitochondria from the axon of a rat neuron. (D) Mitochondria from testis from Drosophila melanogaster. Scale bars: $250 \mathrm{~nm}$. Recordings provided by Felix Lange, Max Planck Institute for Biophysical Chemistry, Göttingen.

In the 1960s, it was common to see cristae as large infolds that exhibit wide openings to the intermembrane space. Daems and Wisse questioned this idea in 1966 and proposed that lamellar cristae are connected to the inner boundary membrane by small tubular structures and named them "pediculi cristae" (Daems and Wisse, 1966). Later, several studies focusing on the fold of the inner membrane using electron tomography confirmed this idea of small openings in the inner boundary membrane, and the "pediculi cristae" were re-named crista junctions (Frey et al., 2002; Lea and Hollenberg, 1989; Mannella et al., 1994; Perkins et al., 1997). Crista junctions usually adopt circular or slightly elongated shapes with a diameter of about $25 \mathrm{~nm}$ (Frey et al., 2002). Data recorded by fluorescence microscopy and immunogold electron microscopy demonstrated that the confined openings function as diffusion barriers and established today's view of cristae as submitochondrial compartments (Vogel et al., 2006; Wolf et al., 2019; Wurm and Jakobs, 2006).

Cristae are key for the metabolic function of mitochondria since the cristae membrane harbors the protein complexes that perform OXPHOS. In mammals, the OXPHOS system consists of the respiratory complexes I-V (complex I does not exist in yeast). Core proteins of each respiratory complex facilitate their catalytic activity, whereas many additional subunits control, for example, the assembly or stability of the complexes (Kadenbach, 2012; Signes and Fernandez-Vizarra, 2018). The assembly of the OXPHOS complexes is an intricate process because most of the subunits are imported from the cytosol, whereas few subunits are mtDNAencoded. The latter are usually hydrophobic proteins whose translation takes place in close proximity to the inner membrane (Mai et al., 2016). The respiratory chain complexes release the energy from $\mathrm{NADH}$ and $\mathrm{FADH}_{2}$ in three steps and transfer protons across the inner 
membrane into the intermembrane space. These three steps are performed by the NADH dehydrogenase (complex I), the cytochrome bc1 complex (complex III) and the cytochrome oxidase (COX, complex IV), which also converts oxygen into water (Kadenbach, 2012). The chemiosmotic hypothesis (Mitchell, 1961) proposes that the translocation of protons generates an electrochemical gradient that can be dissipated by the $\mathrm{F}_{1} \mathrm{~F}_{\mathrm{O}}$-ATP synthase (complex V) for ATP regeneration (Kühlbrandt, 2019; Senior et al., 2002). However, recent studies question the significance of this proton gradient and instead suggest that kinetic coupling of the respiratory chain with the $\mathrm{F}_{1} \mathrm{~F}_{\mathrm{O}}$-ATP synthase drives ATP synthesis (Toth et al., 2020). Nevertheless, the function of the cristae as OXPHOS complex-enriched mitochondrial subcompartments strongly supports the function of the respiratory machinery.

\subsection{Determinants of the Fold of the Inner Membrane}

In 2001, a study observed that in isolated yeast mitochondria, crista junctions would reappear after being ruptured by large-amplitude swelling and recontraction of the matrix (Mannella et al., 2001). The authors suggested that crista junctions could form spontaneously between the inner boundary membrane and the cristae membrane. Subsequently, several theoretical studies asked whether cristae and crista junctions are actively formed, for example by proteins, or if their formation can simply be explained by thermodynamics. One of these studies concluded that the outer membrane, by providing a confined space, supports the invagination of the inner membrane and thereby the formation of cristae and crista junctions (Renken et al., 2002). Nevertheless, calculations suggested that the formation of cristae must be additionally controlled by proteins because the preferred invagination for a protein-free lipid membrane would be a large balloon-like infold that is usually not observable in mitochondria (Ghochani et al., 2010). The same study also predicted that the formation of crista junctions requires membrane bending proteins. Detailed calculations suggested that the formation of the tubular extensions that connect the sheet-like cristae to the inner boundary membranes requires proteinmediated tensile forces of about $20 \mathrm{pN}$ to bend the membranes into their tubular shape (Ghochani et al., 2010).

Historically, also the anionic lipid cardiolipin was considered to shape the inner membrane due to its cone-shaped geometry. In fact, the lipid packing in artificial cardiolipin-containing membranes can be manipulated by $\mathrm{pH}$ modulation, thereby inducing cristae-like invaginations of the membrane (Khalifat et al., 2008, 2011). Vice versa, a study demonstrated that cardiolipin molecules accumulate in curved membrane segments, suggesting that cardiolipin promotes and stabilizes membrane curvature (Beltrán-Heredia et al., 2019). The formation of cristae indeed requires the establishment and maintenance of strong positive and negative membrane curvature (Cogliati et al., 2016). Positively curved membranes (that bulge towards the matrix space, Figure 7), are necessary at the rims of cristae where the cristae membrane performs a $180^{\circ}$ turn. On standard transmission electron micrographs, which show only a thin section of 
mitochondria, the corresponding areas would be the "tips" of cristae (Rabl et al., 2009). The situation is more complicated for the crista junctions as the inner membrane performs a $90^{\circ}$ turn towards the matrix where the inner boundary membrane passes into the cristae membrane. This leads to a strong negative curvature (membrane bulges away from the matrix) of the inner membrane in this area. At the same time, the tubular extensions that connect the membrane pores with the larger, often more sheet-like cristae, exhibit a positive membrane curvature like the rims of the cristae.

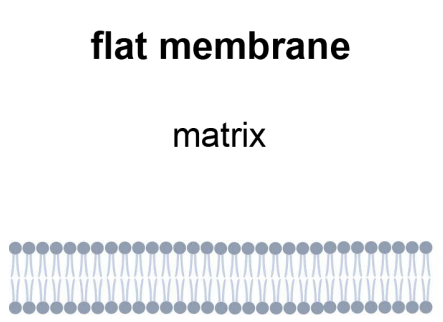

IMS

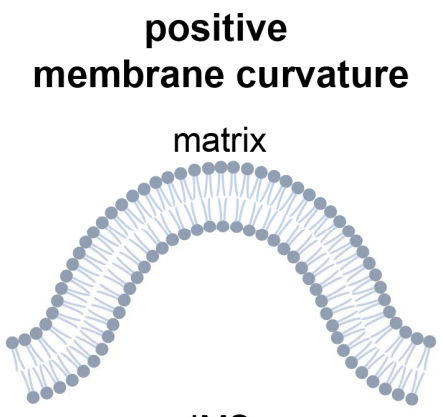

IMS

\section{negative membrane curvature}

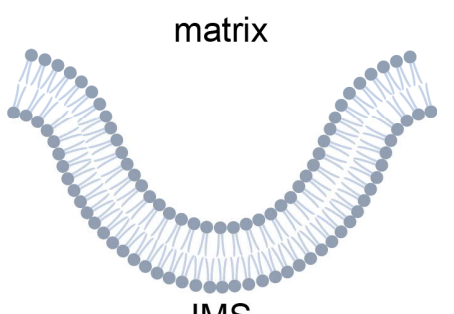

IMS

Figure 7: Membrane curvature in mitochondria. The mitochondrial inner membrane exhibits areas with strong membrane curvature. For the definition of membrane curvature in mitochondria, the matrix space is used as a reference. Positive membrane curvature describes the bulging of the bilayer towards the matrix (the monolayer facing the matrix bends in the direction from the head groups towards the tails). Negative curvature describes the opposite situation when the membrane bulges away from the matrix. IMS: Intermembrane space.

Corroborating the predictions of several theoretical studies, experimental studies have discovered several different membrane-shaping proteins that influence cristae formation (Cogliati et al., 2016; Kondadi et al., 2019; Rabl et al., 2009; Rampelt et al., 2017a). The following section will introduce the most relevant proteins of this class: the dimeric $\mathrm{F}_{1} \mathrm{~F}_{\mathrm{O}}$-ATP synthase, the dynamin-like GTPase OPA1 and the mitochondrial contact site and cristae organizing system (MICOS).

\subsubsection{Dimeric $F_{1} F_{0}-A T P$ synthase}

In recent years, the mitochondrial $\mathrm{F}_{1} \mathrm{~F}_{\mathrm{O}}$-ATP synthase has been identified as one of the protein complexes that strongly influence the shape of the inner membrane. Cryo-electron microscopy (cryo-EM) has confirmed that all known mitochondrial $\mathrm{F}_{1} \mathrm{~F}_{\mathrm{O}}$-ATP synthases form dimers (Kühlbrandt, 2019) which were first observed using blue native polyacrylamide gel electrophoresis (BN-PAGE). Their formation depends on specific subunits that are associated with the membrane-spanning $\mathrm{F}_{\mathrm{O}}$-subunit. In yeast, these subunits are the proteins $\mathrm{Su}$ e, $\mathrm{Su} \mathrm{g}$, and Su k (Arnold et al., 1998). Recent advances in cryo-EM have allowed for the analysis of the precise structures of dimeric mitochondrial $\mathrm{F}_{1} \mathrm{~F}_{\mathrm{O}}$-ATP synthases from different species $(\mathrm{Gu}$ et al., 2019; Hahn et al., 2016; Mühleip et al., 2016; Murphy et al., 2019). One class of dimeric 
$\mathrm{F}_{1} \mathrm{~F}_{\mathrm{O}}$-ATP synthases includes those of fungi like yeast and animals like cows and pigs (Davies et al., 2014; Gu et al., 2019; Kühlbrandt, 2019). The human $\mathrm{F}_{1} \mathrm{~F}_{\mathrm{O}}$-ATP synthase likely also belongs to this class. However, a high-resolution structure of the human protein has not been solved (Davies et al., 2011). The $\mathrm{F}_{1} \mathrm{~F}_{\mathrm{O}}$-ATP synthase dimers of this class generally feature a Vshape and a highly curved membrane-binding domain since the monomers are arranged at an angle of about $90^{\circ}$ (Figure 8 ).

A

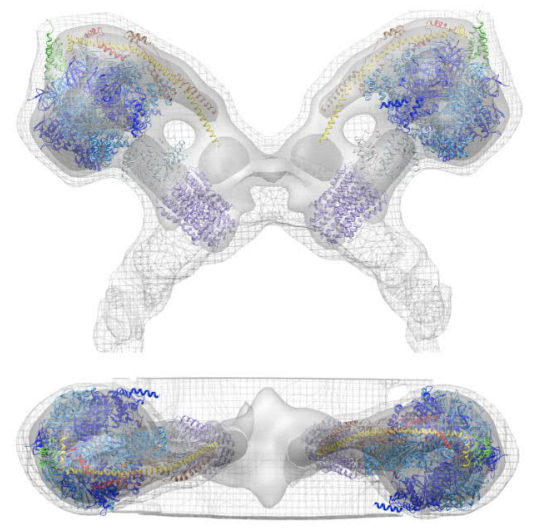

B

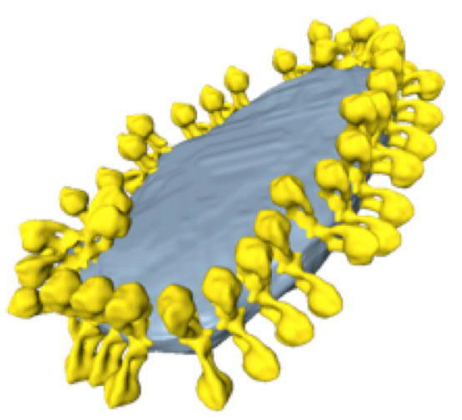

Figure 8: Structure of the dimeric $\mathrm{F}_{1} \mathrm{~F}_{\mathrm{O}}$-ATP synthase from Saccharomyces cerevisiae. (A) Subtomogram average of the ATP synthase dimer from yeast. The $F_{1} F_{O}-A T P$ synthase monomers interact within the membrane and form a V-shaped dimer. (B) Localization of dimers of the $F_{1} F_{O}-A T P$ synthase in isolated mitochondrial membranes. The dimers form elongated rows that stabilize the cristae ridges. Adapted with permission from: Davies et al., 2014.

A second class of $\mathrm{F}_{1} \mathrm{~F}_{\mathrm{O}}$-ATP synthase dimers was found in mitochondria of unicellular green algae like Polytomella sp. (Figure 9). Despite some structural differences, these dimers also exhibit a similar V-shape (Kühlbrandt, 2019). Accordingly, cryo-EM data of both dimer classes demonstrated very similar distributions of the $\mathrm{F}_{1} \mathrm{~F}_{\mathrm{O}}$-ATP synthase dimers on cristae and on membrane vesicles. The dimers form rows or ribbons (Figure 8) that introduce strong positive membrane curvature and thereby stabilize the rims of cristae or vesicles (Blum et al., 2019; Daum et al., 2013; Davies et al., 2012; Dudkina et al., 2005; Strauss et al., 2008).

Mitochondria from the ciliate Paramecium tetraurelia exhibit a third, structurally different type of $\mathrm{F}_{1} \mathrm{~F}_{\mathrm{O}}$-ATP synthase dimers, which instead feature a U-shape. In these dimers, the monomers are positioned nearly in parallel, leading to a less curved membrane-binding domain. Accordingly, the cristae in these ciliate mitochondria form thin tubules that are decorated with helical arrays of the $\mathrm{F}_{1} \mathrm{~F}_{\mathrm{O}}$-ATP synthase dimers (Mühleip et al., 2016).

The formation of dimer rows of the $\mathrm{F}_{1} \mathrm{~F}_{\mathrm{O}}$-ATP synthase is critical for the maintenance of properly shaped cristae. Disassembly of the dimers has been observed during aging and has been associated with the formation of unstructured, balloon-shaped cristae (Daum et al., 2013). In addition, a depletion of the dimerization subunits has strong negative effects on the shape of 
the inner membrane in yeast. Ablation of Su e or Su g induces the loss of cristae "tips" and leads to the formation of septate mitochondria or mitochondria that contain multiple concentric inner membrane layers (“onion-shaped" mitochondria) (Davies et al., 2012; Harner et al., 2016; Paumard et al., 2002; Rabl et al., 2009). The structural changes were found to be accompanied by a drop of the membrane potential and reduced growth (Bornhövd et al., 2006), suggesting that a proper inner membrane architecture is essential for the metabolic function of mitochondria (Davies et al., 2018; Toth et al., 2020).
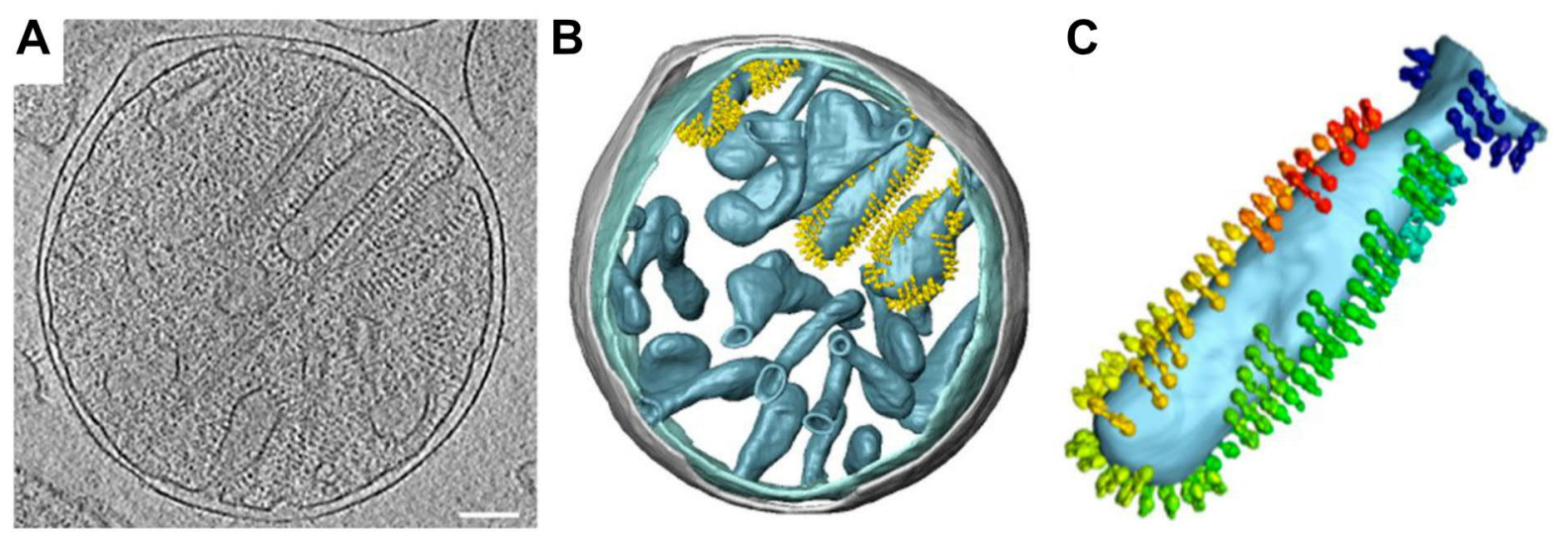

Figure 9: Rows of $F_{1} F_{0}-A T P$ synthase dimers stabilize cristae. (A) Cryo-electron tomography (Cryo-ET) recording of an isolated mitochondrion from Polytomella sp.. (B) 3D reconstruction of the mitochondrion shown in (A). $F_{1} F_{O}-A T P$ synthase dimers (yellow) are shown on 3 individual cristae. The inner membrane is displayed in light blue, the outer membrane is displayed in grey. (C) Individual club-shaped crista from the mitochondrion in $(A)$. The dimers of the $F_{1} F_{O}$-ATP synthase (shown in rainbow color) are located at the cristae edges with positive membrane curvature. Scale bar: $100 \mathrm{~nm}$. Adapted with permission from: Blum et al., 2019.

\subsubsection{OPA1 and Mgm1}

Mitochondrial inner membrane fusion depends on Mgm1 in yeast (Meeusen et al., 2006; Sesaki et al., 2003; Wong et al., 2000) and OPA1 in mammals (Lee et al., 2004; Pernas and Scorrano, 2016; Youle and Bliek, 2012). Both proteins are dynamin-like GTPases that are considered homologs, although their actual homology is only about 33\% (Votruba, 2004). Cooperation of these GTPases with the outer membrane fusion machinery allows for efficient fusion of mitochondrial tubules, although outer membrane fusion is also possible in the absence of Mgm1 (Cipolat et al., 2004; Hales and Fuller, 1997; Hermann et al., 1998; Meeusen et al., 2004, 2006). Depletion of Mgm1 is characterized by severe fragmentation of the mitochondrial network, decreased respiration rates, a loss of mtDNA and disturbed lipid homeostasis (Guan et al., 1993; Jones and Fangman, 1992; Kojima et al., 2019; Meeusen et al., 2006; Shepard and Yaffe, 1999). Mutations in OPA1 are associated with optic atrophy, a condition caused by the degeneration of the optic nerve (Alexander et al., 2000; Delettre et al., 2000). Depletion of OPA1 causes, similar to Mgm1 depletion, massive fragmentation of mitochondrial networks (Cipolat et al., 2004; Ishihara et al., 2006). 
In addition, numerous studies observed a disturbed inner membrane architecture upon ablation of Mgm1/OPA1, supporting a critical role of these proteins in inner membrane shaping (Cogliati et al., 2016; Giacomello et al., 2020; Harner et al., 2016). For both proteins, the ability to shape the inner membrane generally depends on their oligomerization (Frezza et al., 2006; Meglei and McQuibban, 2009). Both Mgm1 and OPA1 exist as membrane-bound, long isoforms (l-isoforms) and soluble, short isoforms (s-isoforms), which are generated by proteolytic cleavage of 1-Mgm1 (Herlan et al., 2004; McQuibban et al., 2003; Sesaki et al., 2003) and l-OPA1, respectively (Griparic et al., 2007; Ishihara et al., 2006; Song et al., 2007). It is widely accepted that the proteolytic cleavage of 1-Mgm1/1-OPA1 regulates the fusion process in yeast (Esser et al., 2002; Herlan et al., 2003) and mammals (Dotto et al., 2018; Duvezin-Caubet et al., 2006; MacVicar and Langer, 2016). Nevertheless, diverging results were published regarding the precise function of the short and long isoforms of Mgm1/OPA1. Both isoforms of Mgm1 are required for fusion, but the GTPase activity is only essential for the function of s-Mgm1, suggesting that 1-Mgm1 can act as a membrane anchor that links the soluble s-Mgm1 to the inner membrane (DeVay et al., 2009; Herlan et al., 2004; Sesaki et al., 2003; Zick et al., 2009).

Different assumptions exist regarding the function of the short and long isoforms of OPA1. It has been reported that s-OPA1 is necessary for maintenance of the cristae morphology but dispensable for fusion (Anand et al., 2014; Lee et al., 2017), suggesting different physiological roles of short and long OPA1 isoforms. However, recent in vitro data suggest that, similar to the findings in yeast, both OPA1 isoforms cooperate to achieve membrane fusion. Whereas 1OPA1 generally enables membrane docking and hemifusion of liposomes, stoichiometric levels of s-OPA1 are required to enhance efficient membrane fusion and pore opening in vitro (Ge et al., 2020).

Recently, crystal structures of s-Mgm1 from Saccharomyces cerevisiae (Yan et al., 2020) and from Chaetomium thermophilum (Faelber et al., 2019) have provided new insights into the function of the proteins. In both organisms, the monomers of s-Mgm1 have a very similar structure to the GTPase dynamin, yet, different oligomeric states of s-Mgm1 were reported in both studies. In yeast, Yan and colleagues discovered a nucleotide-independent trimeric s-Mmg1 complex that was also detectable in aqueous solution during analytical ultracentrifugation. The authors suggested a model in which assemblies of s-Mgm1 trimers induce small inner membrane tips that could finally allow inner membrane fusion (Yan et al., 2020).

In contrast, purified s-Mgm1 from Chaetomium thermophilum crystalized as a dimer and, when incubated with liposomes, assembled into regular filamentous structures that induced the tubulation of the liposomes (Faelber et al., 2019). Cryo-EM revealed that, on the outer leaflet of these membrane tubes, s-Mgm1 tetramers assembled into a 4-start left-handed helix with a radius of about $20 \mathrm{~nm}$ and a pitch of about $50 \mathrm{~nm}$ (Faelber et al., 2019). 
Surprisingly, tubulation could also be caused by s-Mgm1 tetramers assembling into extended patches on the inner surface of such lipid vesicles (Figure 10). Although these assemblies formed nucleotide-independently, Faelber and colleagues reported evidence for a GTPdependent rearrangement of Mgm1 on the surfaces of lipid tubes (Faelber et al., 2019). The authors proposed that Mgm1 forms left-handed helical arrangements inside crista junctions that constrict the junctions in a GTP-dependent manner.

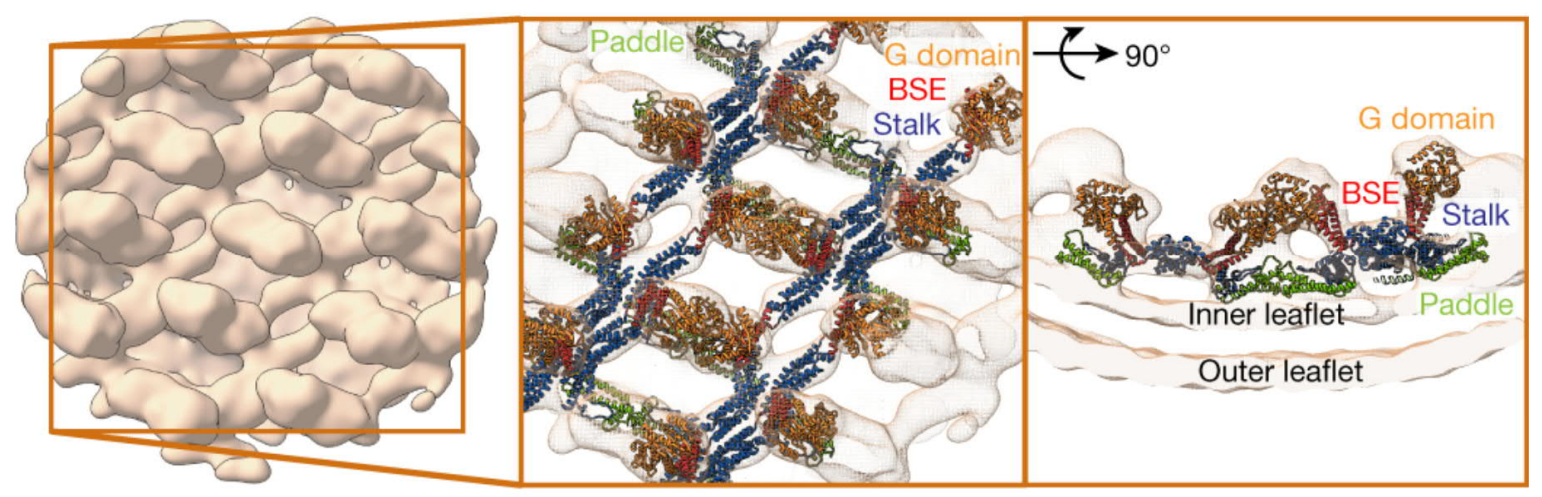

Figure 10: s-Mgm1 decorating the inner surface of lipid tubes. Cryo-electron tomography of Mgm1. Left: The subtomogram average shows regular Mgm1-assemblies on the inner surface of a membrane tube. Middle: Magnification shows the structure of Mgm1 fitted into the cryo-ET volume. Right: Rotated view shows the Mgm1 tetramer decorating the inner leaflet of the membrane tube. Abbreviations: G-domain: GTPase domain, BSE: Bundle signaling element. Adapted with permission from: Faelber et al., 2019.

Purified s-Mgm1 from both yeast and from Chaetomium thermophilum interacts with negatively charged lipids like cardiolipin or phosphatidylserine (Faelber et al., 2019; Yan et al., 2020). Lipid binding of Mgm1 strongly increases the GTPase activity, which is in line with various findings that cardiolipin promotes activity and assembly of Mgm1 and OPA1 (Ban et al., 2010; DeVay et al., 2009; Faelber et al., 2019; Yan et al., 2020). Combined with recent structural data, these findings demonstrate the ability of Mgm1/OPA1 oligomers to modulate the shape of the inner membrane.

\subsubsection{MICOS}

The mitochondrial contact site and cristae organizing system (MICOS; previously also named as mitochondrial inner membrane organizing system (MINOS) or mitochondrial organizing structure (MitOS)) is a large heterooligomeric protein complex that is embedded in the mitochondrial inner membrane at crista junctions (Alkhaja et al., 2011; Harner et al., 2011; Hoppins et al., 2011; Malsburg et al., 2011). MICOS consists of at least 6 different subunits in yeast and 7 different subunits in mammals (Laan et al., 2016; Rampelt et al., 2017a). For simplification, all MICOS proteins have been (re)named according to a uniform nomenclature (Pfanner et al., 2014). In yeast, the MICOS complex comprises Mic10, Mic12, Mic19, Mic26, 
Mic27 and Mic60, whereas in mammals it is formed by Mic10, Mic13, Mic19, Mic25, Mic26, Mic27 and Mic60 (Friedman et al., 2015; Guarani et al., 2015; Laan et al., 2016).

The depletion of several MICOS subunits causes the disruption of crista junctions and an accumulation of detached cristae in the matrix (Alkhaja et al., 2011; Harner et al., 2011; Hoppins et al., 2011; Malsburg et al., 2011; Rabl et al., 2009) (Figure 11). In yeast, this phenotype is particularly strong upon loss of Mic60 or Mic10, which are the core subunits of the MICOS complex (Laan et al., 2016). Both proteins are highly conserved across species and recent studies have shown that Mic60 is an ancient protein with orthologues in $\alpha$-proteobacteria (Huynen et al., 2015; Muñoz-Gómez et al., 2017). Remarkably, these $\alpha$-proteobacterial Mic60 orthologues could be associated with inner membrane invaginations, suggesting an endosymbiotic origin of the mitochondrial cristae (Huynen et al., 2015; Muñoz-Gómez et al., 2015).

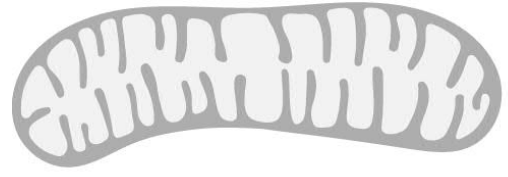

Wildtype

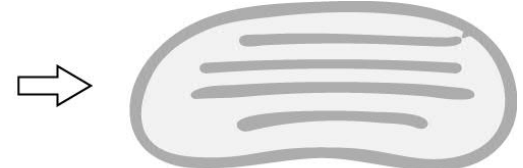

Loss of MICOS

Figure 11: MICOS determines the fold of the inner membrane. Cartoon illustrating the morphological changes of the mitochondrial membrane architecture upon loss of MICOS. MICOSdeficient mitochondria show a strong reduction in the number of crista junctions and an aberrant inner membrane architecture.

Several studies confirmed that MICOS contains two subcomplexes, which were named after Mic60 and Mic10. The constituents of the two subcomplexes vary between lower and higher eukaryotes. In mammals, the Mic60-subcomplex is formed by Mic60, Mic19 and Mic25, whereas the Mic10-subcomplex consists of Mic10, Mic13, Mic26 and Mic27 (Anand et al., 2016; Guarani et al., 2015; Li et al., 2015; Ott et al., 2012; Xie et al., 2007). In yeast, the Mic60subcomplex consists of Mic60 and Mic19 and the Mic10-subcomplex is formed by Mic10, Mic12, Mic26 and Mic27 (Bohnert et al., 2015; Friedman et al., 2015). The precise function of the two subcomplexes is not yet understood, but it became clear that a complicated regulatory interplay between the different subunits in each subcomplex exists (Guarani et al., 2015; Harner et al., 2011; Hoppins et al., 2011; Malsburg et al., 2011).

Since its discovery in 2011, several distinct functions of MICOS have been identified. These functions include the formation of crista junctions, the formation of contact sites between the inner and outer membrane, and the shaping of the inner membrane.

\subsubsection{MICOS regulates contact site formation}

MICOS undergoes numerous interactions with a variety of proteins which are crucial for the maintenance of the mitochondrial architecture and function (Giacomello et al., 2020; Hoppins et al., 2011; Laan et al., 2016). The paralogs Mic19 and Mic25 are peripheral inner membrane 
proteins that are part of the Mic60-subcomplex (Harner et al., 2011; Hoppins et al., 2011; Malsburg et al., 2011; Ott et al., 2012; Xie et al., 2007). In yeast and human mitochondria, Mic19 regulates the assembly of the MICOS complex (Friedman et al., 2015; Sakowska et al., 2015). Moreover, it mediates stable contact sites between Mic60 and the SAM complex in the outer membrane, leading to the formation of a mitochondrial intermembrane space bridging (MIB) complex (Huynen et al., 2015; Ott et al., 2012, 2015; Tang et al., 2018; Xie et al., 2007; Zerbes et al., 2012). The importance of the MIB complex is underscored by the fact that a disruption of the Mic60-Mic19-SAM50 axis, either by SAM50 or Mic19 depletion, causes a loss of crista junctions and a disturbed cristae architecture (Körner et al., 2012; Ott et al., 2012, 2015). These observations suggest that contact site formation and crista junction formation are closely related processes.

In yeast, different experimental approaches verified further interactions of Mic60 with outer membrane proteins like Porin or the TOM complex (Hoppins et al., 2011; Körner et al., 2012; Malsburg et al., 2011; Zerbes et al., 2012). The interaction of Mic60 with the protein import machinery promote efficient protein biogenesis but do not require other MICOS subunits (Bohnert et al., 2012; Höhr et al., 2014), suggesting that Mic60, next to contact site formation, fulfils a diverse set of functions in mitochondria.

\subsubsection{MICOS shapes the inner membrane}

The important role of Mic60 in inner membrane shaping was recognized several years before the discovery of MICOS. Knockdown experiments first demonstrated that Mic60 is essential to maintain both crista junctions and the fold of the inner membrane in mammals (John et al., 2005). Similar results were later also reported in other species (Mun et al., 2010; Rabl et al., 2009; Xie et al., 2007). Rabl and colleagues further described that overexpression of Mic60 induced branching of cristae in yeast, supporting the hypothesis that Mic60 could stabilize negative membrane curvature to support crista junction formation (Rabl et al., 2009). Indeed, recent studies have confirmed that Mic60 proteins from both Saccharomyces cerevisiae and from Chaetomium thermophilum exhibit membrane-shaping abilities. For instance, purified Mic60 from yeast causes the tubulation of lipid vesicles when it is incorporated into artificial membranes (Figure 12). Furthermore, fusion constructs of Mic60 with the maltose binding protein induce the formation of membrane invaginations when expressed in E. coli (Hessenberger et al., 2017; Tarasenko et al., 2017).

Mic10, the core subunit of the Mic10-subcomplex, exhibits very similar membrane-shaping abilities to Mic60 as it causes membrane bending when it is integrated into artificial membranes (Barbot et al., 2015). Likewise, overexpression of Mic10 induces the formation of aberrant cristae and crista junctions in yeast (Bohnert et al., 2015). Membrane bending caused by Mic10 is driven by the oligomerization of the protein (Barbot et al., 2015; Bohnert et al., 2015) which features a hairpin-like structure and encompasses two transmembrane helices that are connected 
by a short loop. Conserved glycine-rich GxGxGxG motifs within the two transmembrane domains of the V-shaped Mic10 monomers can mediate the formation of exceptionally stable membrane-bending oligomers (Barbot et al., 2015; Bohnert et al., 2015).

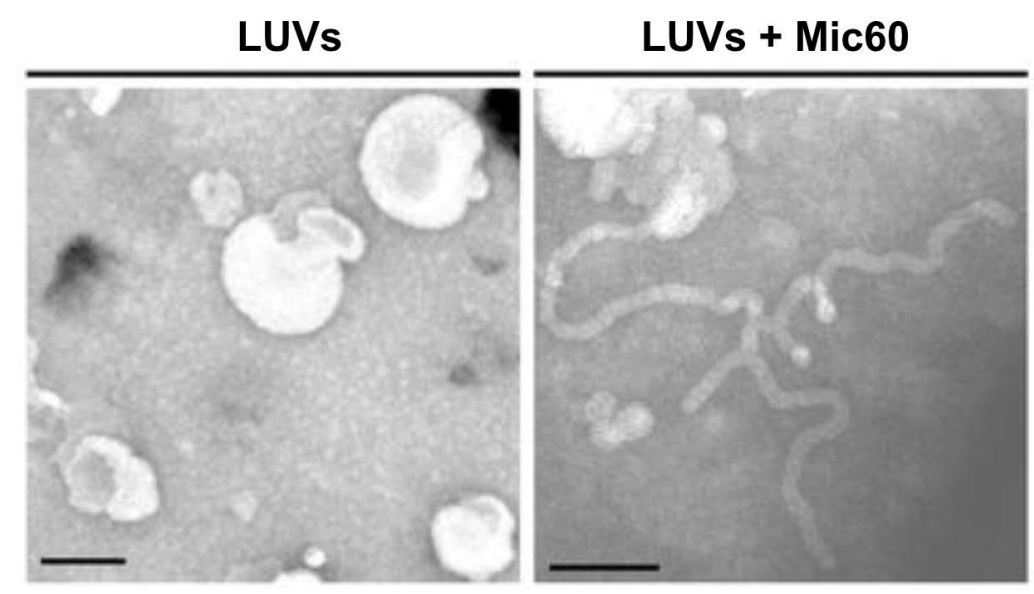

Figure 12: Mic60 shows membrane-bending activity. Mic60 from Saccharomyces cerevisiae was purified from E. coli and incorporated into large unilamellar vesicles (LUVs). Images show electron micrographs of LUVs and LUVs after incorporation of Mic60. Scale bar: $100 \mathrm{~nm}$. Adapted with permission from: Tarasenko et al., 2017.

Mic10 is functionally related to the remaining subunits of the Mic10-subcomplex, namely Mic12/13, Mic26 and Mic27 (Table 1). A complex regulatory interplay exists between these proteins, as they exhibit a coordinated expression that differs between yeast and mammals. In mammals, Mic13 controls the expression levels of Mic10 and has been suggested to be the linker between the two MICOS subcomplexes (Guarani et al., 2015). Similarly, Mic12 links the MICOS subcomplexes in yeast but does not influence the expression levels of Mic10 (Zerbes et al., 2016). Mic10, however, controls the protein levels of Mic12 and Mic27 in yeast (Harner et al., 2011; Hoppins et al., 2011; Malsburg et al., 2011) while Mic27, in turn, stabilizes Mic10 oligomers (Bohnert et al., 2015; Zerbes et al., 2016).

Both, Mic27 and Mic26, belong to the group of Apolipoprotein O like proteins (ApoO) and also exhibit coordinated expression (Koob et al., 2015). Mic27 has the ability to directly bind cardiolipin (Weber et al., 2013), suggesting a functional connection between lipids and the Mic10-subcomplex. Indeed, a study in yeast demonstrated that the Mic10-subcomplex assembles in a cardiolipin- and OXPHOS-dependent manner, whereas the Mic60-subcomplex assembles independently from these factors (Friedman et al., 2015). As cardiolipin can induce or support membrane curvature, it might facilitate the recruitment and assembly of the Mic10subcomplex to intensify and stabilize membrane curvature at crista junctions (Rampelt et al., 2017a). 
Table 1: Overview of MICOS subunits. This table shows the known MICOS subunits, their predicted membrane topology, and their reported functions. The table also provides alternatively used names of the proteins. Adapted from: Rampelt et al., 2017a.

\begin{tabular}{|c|c|c|c|c|}
\hline & Name & $\begin{array}{l}\text { Predicted } \\
\text { topology }\end{array}$ & Reported functions & Alternative names \\
\hline \multirow{3}{*}{ 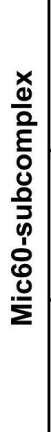 } & Mic60 & & $\begin{array}{l}\text { contact site formation, membrane-shaping, } \\
\text { promotes protein biogenesis and lipid } \\
\text { trafficking }\end{array}$ & $\begin{array}{l}\text { Mitofilin, IMMT, Fcj1, } \\
\text { Aim28, MINOS2 }\end{array}$ \\
\hline & Mic19 & & $\begin{array}{l}\text { regulatory subunit, possibly connecting } \\
\text { MICOS subcomplexes in yeast }\end{array}$ & $\begin{array}{l}\text { CHCHD3, Mcs19, } \\
\text { CHCH-3, MINOS3 }\end{array}$ \\
\hline & Mic25 & & paralog of Mic19 in metazoa & $\mathrm{CHCHD6}$, CHCM1 \\
\hline \multirow{5}{*}{ 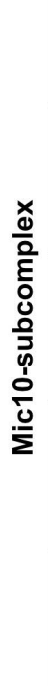 } & Mic10 & & $\begin{array}{l}\text { membrane-shaping, oligomerization, } \\
\text { stabilization of crista junctions }\end{array}$ & $\begin{array}{l}\text { MINOS1, Mio10, } \\
\text { Mcs10, Mos1 }\end{array}$ \\
\hline & Mic12 & & connects MICOS subcomplexes in fungi & Aim5, Fmp51, Mcs12 \\
\hline & Mic13 & & $\begin{array}{l}\text { connects the MICOS subcomplexes in } \\
\text { mammalia, putative Mic12 ortholog in } \\
\text { metazoa }\end{array}$ & QIL1 \\
\hline & Mic26 & & $\begin{array}{l}\text { paralog of Mic27, putative lipid-binding } \\
\text { protein }\end{array}$ & $\begin{array}{l}\text { ApoO, Mio27, Mcs } 29 \\
\text { Mos2, Mic23 in } \\
\text { metazoa }\end{array}$ \\
\hline & Mic27 & $\frac{1 \mathrm{Mix}}{\mathrm{rix}}$ & $\begin{array}{l}\text { lipid-binding protein, stabilizes Mic10 } \\
\text { oligomers, mediates interaction of MICOS } \\
\text { and ATP synthase }\end{array}$ & $\begin{array}{l}\text { APOOL, Aim37, Mcs27, } \\
\text { MOMA-1, Mic28 }\end{array}$ \\
\hline
\end{tabular}

\subsection{Interplay of Membrane-shaping Proteins}

In 2009, Rabl and colleagues proposed that cristae formation depends on an antagonism between Mic60 and the dimeric $\mathrm{F}_{1} \mathrm{~F}_{\mathrm{O}}$-ATP synthase. Their model of cristae biogenesis predicted that Mic60, by stabilizing negative membrane curvature, forms the crista junctions whereas dimers of the $\mathrm{F}_{1} \mathrm{~F}_{\mathrm{O}}$-ATP synthase shape the positively curved cristae rims. This idea was supported by the inner membrane architecture upon depletion of Mic60 or of the ATP synthase subunit $\mathrm{Su}$ e and by the observation that Mic60 could negatively influence the oligomeric state of the $\mathrm{F}_{1} \mathrm{~F}_{\mathrm{O}}$-ATP synthase (Rabl et al., 2009). Later, crosslinking experiments in yeast refined the idea of a functional interplay between the $\mathrm{F}_{1} \mathrm{~F}_{\mathrm{O}}$-ATP synthase and MICOS as they showed a direct physical interaction between the dimeric $\mathrm{F}_{1} \mathrm{~F}_{\mathrm{O}}$-ATP synthase and Mic10 (Eydt et al., 2017; Rampelt et al., 2017b) (Figure 13). In addition, Eydt and colleagues demonstrated that Mic27 can promote the oligomerization of the dimeric $\mathrm{F}_{1} \mathrm{~F}_{\mathrm{O}}$-ATP synthase, suggesting an intricate interplay between the Mic10-subcomplex and the $\mathrm{F}_{1} \mathrm{~F}_{\mathrm{O}}$-ATP synthase in cristae formation (Eydt et al., 2017). 
Fungi

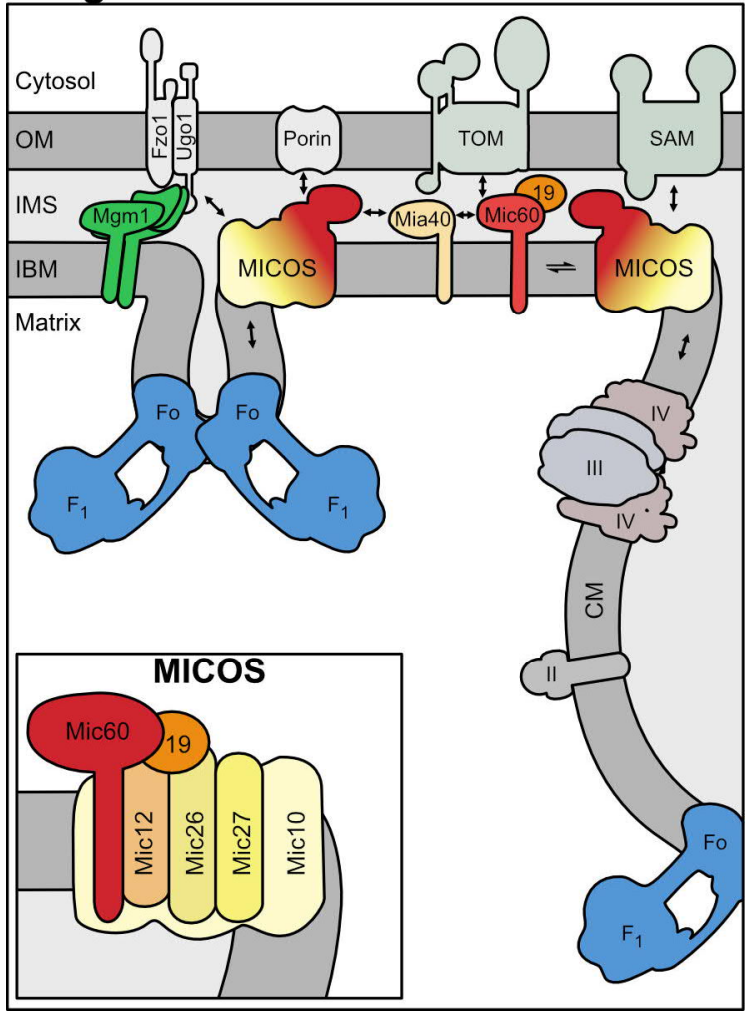

Mammals

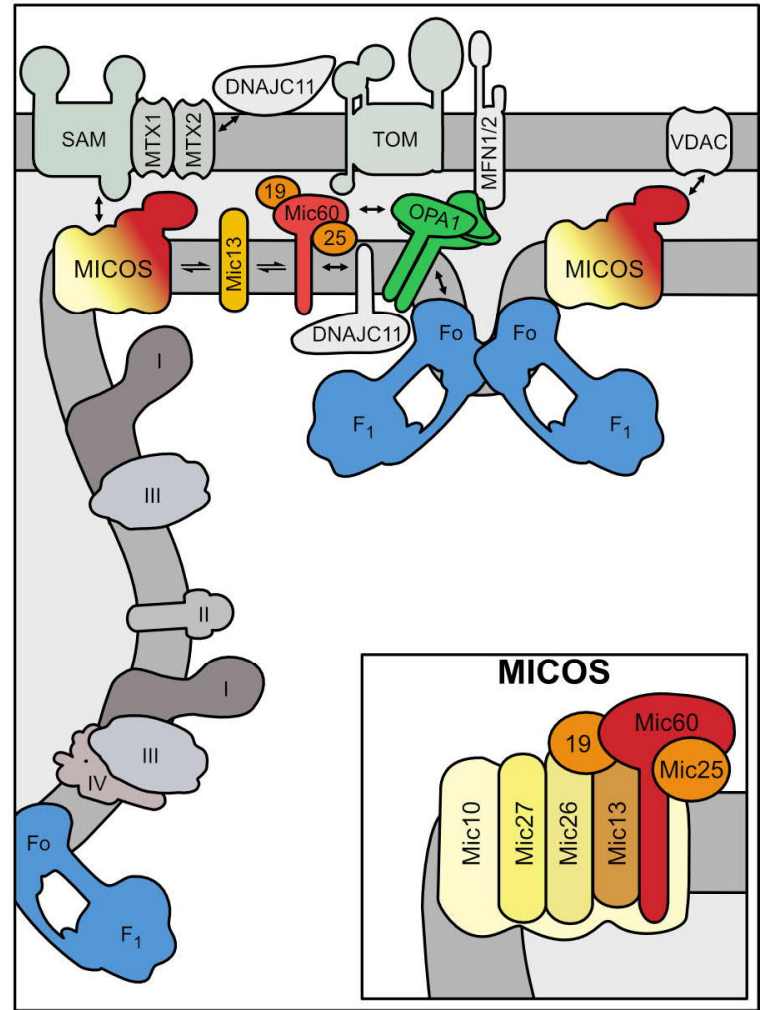

Figure 13: MICOS has a multitude of interaction partners. The illustration summarizes differences between fungi (left) and mammals (right) in terms of protein composition and proteinprotein interactions in the mitochondrial membranes. In fungi, as well as in mammals, MICOS interacts with a multitude of proteins in both mitochondrial membranes. Central key players in cristae formation are highlighted. Blue: $F_{1} F_{O}-A T P$ synthase, green: Mgm1/ OPA1, red: MICOS. Adapted from: Van der Laan et al., 2016.

MICOS also interacts physically with OPA1, as demonstrated by cross-linking and complex immunoprecipitation experiments (Barrera et al., 2016; Glytsou et al., 2016). However, immunogold EM has suggested that OPA1, unlike Mic60, is more enriched in the cristae membrane (Barrera et al., 2016). Inconsistent conclusions have been drawn regarding the role of Mic60 and OPA1 in crista junction formation. Barrera and colleagues observed that OPA1 depletion disturbed the cristae architecture but did not significantly influence the number of crista junctions (Barrera et al., 2016). Accordingly, the authors concluded that OPA1 controls crista morphology but is dispensable for crista junction formation. Another study came to contradicting conclusions as the authors reported that depletion of both Mic60 and OPA1 reduced the number of crista junctions to a similar extent in mouse cells (Glytsou et al., 2016). Moreover, upon simultaneous depletion of both proteins, the number of crista junctions did not further decrease, suggesting that OPA1 and Mic60 could be part of the same pathway regulating crista junctions (Glytsou et al., 2016). The overexpression of both Mic60 and OPA1 increased the number of crista junctions. However, OPA1 overexpression could not rescue the loss of crista junctions induced by Mic60 ablation, supporting the idea of different functions for the proteins. Mic60 overexpression in OPA1 depleted cells increased the number of crista junctions 
only moderately, compared to the overexpression of Mic60 in wild type cells. In cells overexpressing OPA1, concurrent Mic60 overexpression did not further enhance the number of crista junctions (Glytsou et al., 2016). Therefore, the authors concluded that Mic60 and OPA1 are both involved in crista junction formation with OPA1 being upstream of Mic60 in this function (Giacomello et al., 2020; Glytsou et al., 2016). However, for unknown reasons, the reported reduction in the number of crista junctions upon Mic60 depletion in mouse cells was surprisingly low as compared to previous studies using other species (Rabl et al., 2009). This might indicate limited transferability of these findings to other organisms. Generally, the role of OPA1 in crista junction regulation has been a highly debated issue for years (Yamaguchi and Perkins, 2009). Immunogold EM analysis repeatedly confirmed that OPA1 does not localize specifically at crista junctions as demonstrated for MICOS (Barrera et al., 2016; Griparic et al., 2004; Harner et al., 2011; Olichon et al., 2002). Moreover, despite the fact that overexpression of OPA1 delays cytochrome c release during apoptosis, it has modest influence on the diameter of crista junctions (Frezza et al., 2006; Yamaguchi and Perkins, 2009), raising doubts about the importance of OPA1 for crista junction stability.

OPA1 also physically interacts with the $\mathrm{F}_{1} \mathrm{~F}_{\mathrm{O}}$-ATP synthase and influences its oligomeric state (Banerjee and Chinthapalli, 2014; Patten et al., 2014; Quintana-Cabrera et al., 2018). Overexpression of OPA1 supports the formation of $\mathrm{F}_{1} \mathrm{~F}_{\mathrm{O}}$-ATP synthase oligomers and protects mitochondria from losing the electrochemical gradient upon inhibition of respiratory complex III (Quintana-Cabrera et al., 2018). However, when both the $\mathrm{F}_{1} \mathrm{~F}_{\mathrm{O}}$-ATP synthase and OPA1 were incorporated into proteoliposomes, OPA1 did not promote oligomerization of the $\mathrm{F}_{1} \mathrm{~F}_{\mathrm{O}^{-}}$ ATP synthase (Quintana-Cabrera et al., 2018), suggesting that oligomerization of the $\mathrm{F}_{1} \mathrm{~F}_{\mathrm{O}^{-}}$ ATP synthase could be a secondary effect of OPA1 overexpression. Therefore, future studies will be important to understand the functional connection of the two proteins.

\subsection{Models of Cristae Formation}

Dissecting the interplay of the aforementioned membrane-shaping proteins is essential for understanding the mechanism of mitochondrial cristae formation. Targeting this issue is challenging since biochemistry and electron microscopy only provide snapshots of cells, and thus cannot directly target the dynamic processes of cristae formation. Although several different models of cristae biogenesis have been proposed, the mechanism of cristae formation is still highly debated. The different models suggested so far can generally be differentiated into two major groups. The first group (Figure 14) comprises four different models, which propose different types of inner membrane remodeling to generate cristae. These models are the invagination model, the balloon model, the de-novo vesicle germination model, and the cristae fission-fusion model (Davies et al., 2012; Jiang et al., 2019; Mühleip et al., 2016; Rabl et al., 2009; Zick et al., 2008). 
A

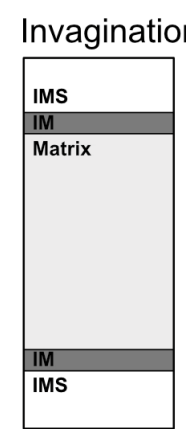

C

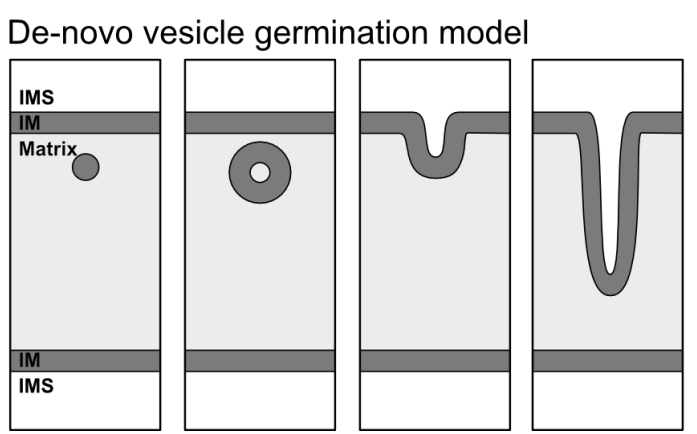

B

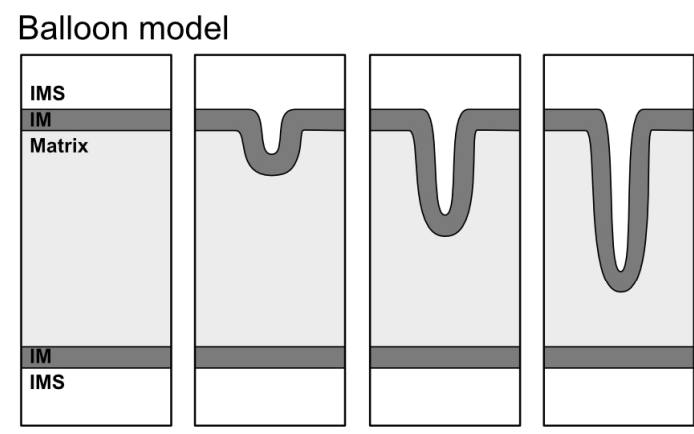

D

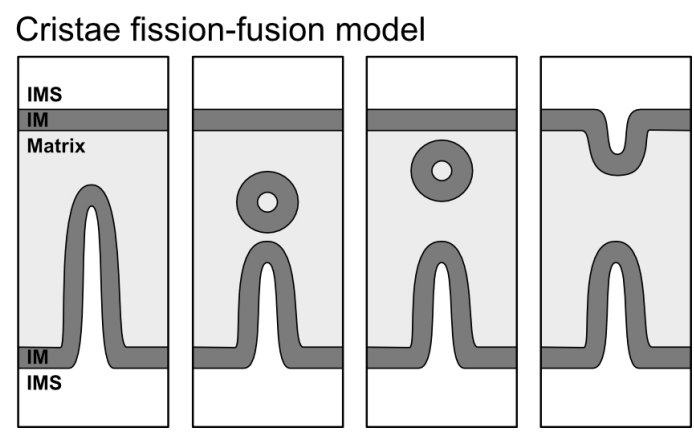

Figure 14: Models of cristae formation. (A-D) Four different models of cristae formation are shown, which predict inner membrane remodeling. (A) In the invagination model, the membrane bends towards the matrix, which is followed by crista junction formation. (B) In the balloon model, crista junction formation precedes the invagination of the membrane. (C) The de-novo vesicle germination model suggests de novo formation of inner membrane vesicles that fuse with the inner boundary membrane. (D) The cristae fission-fusion model predicts fission and fusion of cristae to maintain their structure. The IM is shown in dark gray. The matrix is shown in light grey. Abbreviations: IMS: inner membrane space, IM: inner membrane. Adapted from: Zick et al., 2008.

The invagination model shares high similarity with the balloon model (Figure 14 A and B) as in both models cristae are formed as invaginations of the inner membrane. The models differ in the process of crista junction formation. In the invagination model, crista junction formation follows the membrane invagination and fixes newly formed cristae into position, while in the balloon model the formation of the crista junction precedes the invagination of the inner membrane (Rabl et al., 2009; Zick et al., 2008). The de-novo vesicle germination model (Figure $14 \mathrm{C}$ ) proposes the de novo formation of cristae membrane vesicles in the matrix space that later attach to the inner boundary membrane (Zick et al., 2008). Similarly, the cristae fissionfusion model (Figure 14 D) predicts fission and fusion of the inner membrane to facilitate the formation of the cristae (Kondadi et al., 2020; Zick et al., 2008). A second group of cristae formation models includes the hemifusion and the fusion-remnant models, which suggest that cristae are formed upon mitochondrial tubule fusion (Figure 15). In both models, outer membrane fusion of mitochondrial tubules creates an inner membrane septum that is transformed into a crista by inner membrane remodeling. The membrane remodeling is achieved either by hemifusion or by a zipper-like mechanism which detaches the septum from the inner boundary membrane (Harner et al., 2016; Kojima et al., 2019; Zick et al., 2008). 
A

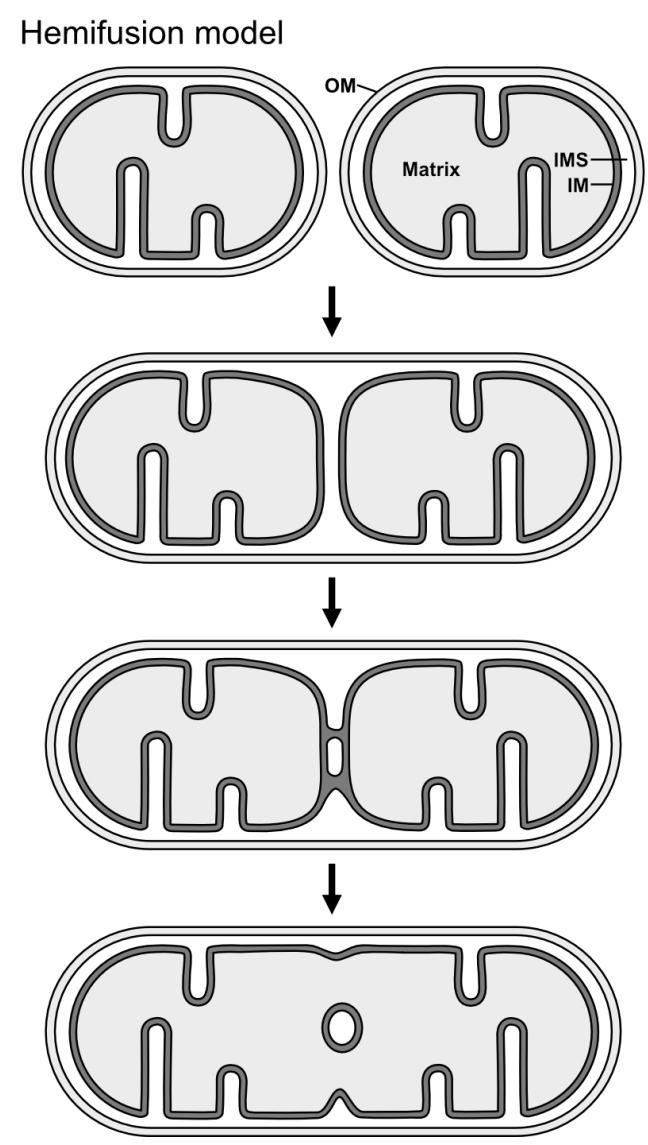

B

Fusion-remnant model
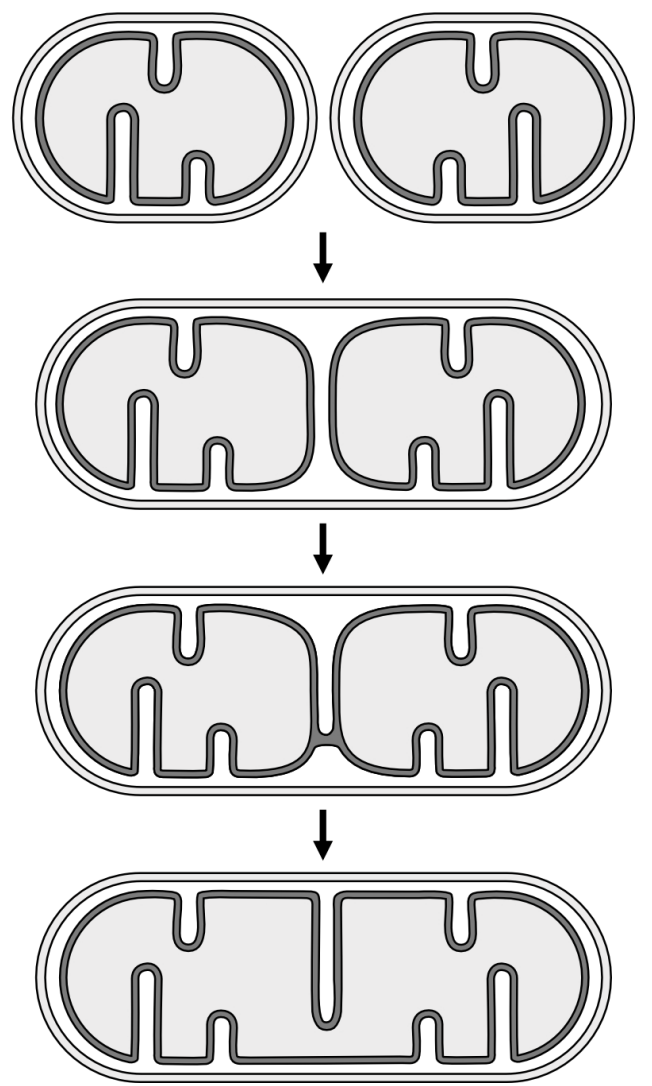

Figure 15: Models of cristae formation following outer membrane fusion. (A-B) Two suggested models of cristae formation are shown, which depend on fusion of mitochondrial tubules. Following outer membrane fusion, a septum is formed by the two juxtapositioned IMs (second panel). (A) In the hemifusion model this septum is transformed into a crista by hemifusion. (B) In the fusion-remnant model, the septum is detached from the IBM and fused to form a lamellar crista. Abbreviations: IM: inner membrane, IMS: inner membrane space, OM: outer membrane. Adapted from: Zick et al., 2008.

To date, none of the aforementioned models of cristae formation is widely accepted as the "general mechanism" of cristae formation. However, different studies have provided data supporting several of the introduced models. Cryo-EM data of dimeric $\mathrm{F}_{1} \mathrm{~F}_{\mathrm{O}}$-ATP synthases that bend membranes by spontaneous row formation greatly support the idea of protein-driven inner membrane invaginations (Blum et al., 2019; Davies et al., 2012; Mühleip et al., 2016). Analysis of mitochondrial maturation in Drosophila melanogaster, in turn, suggested a combination of the balloon model and the de-novo vesicle germination model based on observations of inner membrane structures that are devoid of crista junctions (Jiang et al., 2019). Recently, a study supported the cristae fission-fusion model using 2D super-resolution microscopy (Kondadi et al., 2020). Nevertheless, due to limitations in optical resolution, 2D nanoscopy recordings could not clearly prove fusion or fission of cristae. 
The fact that different experimental data have supported different models of cristae formation might indicate that several of the aforementioned mechanisms have relevance for cristae formation. Remarkably, two studies in yeast provided evidence that at least two independent pathways of cristae formation could exist in parallel (Harner et al., 2016; Kojima et al., 2019). The accordingly developed model of cristae formation (Figure 16) attributes the formation of tubular cristae to an invagination of the mitochondrial inner membrane which is controlled by MICOS and by the assembly of the dimeric $\mathrm{F}_{1} \mathrm{~F}_{\mathrm{O}}$-ATP synthase. Formation of larger lamellar cristae, however, is attributed to the remodeling of an inner membrane septum as described by the fusion-remnant model (Zick et al., 2008). Harner and colleagues suggested that the remodeling of such septa into lamellar cristae is also controlled by MICOS and the dimeric $\mathrm{F}_{1} \mathrm{~F}_{\mathrm{O}}$-ATP synthase, but requires additional interplay with Mgm1 for inner membrane fusion (Harner et al., 2016). Although two independent studies underscored the idea of distinct mechanisms for tubular and lamellar cristae formation in Saccharomyces cerevisiae, this model has not yet been tested in other organisms. Hence, it is unclear if it is also meaningful in higher eukaryotes or if it is specific to Saccharomyces cerevisiae.

Taken together, further experimental data are needed to understand how the mitochondrial inner membrane is shaped. It remains unclear if a general mechanism of cristae formation exists, or if several of the proposed mechanisms exist side-by-side. Furthermore, it remains an open question whether cristae formation is a conserved process across species or if different species exhibit different mechanisms of cristae biogenesis. Therefore, the recently proposed role of outer membrane fusion in cristae formation (Figure 16) must also be validated in mammals. In addition, the precise function and interplay of important inner membrane-shaping proteins remains superficial. It is well known that MICOS is essential for the maintenance of crista junctions and the cristae architecture. However, the function of the two subcomplexes and their interplay in cristae formation is largely unknown. Data from yeast indicated that the Mic10 subcomplex directly interacts with the dimeric $\mathrm{F}_{1} \mathrm{~F}_{\mathrm{O}}$-ATP synthase, but the significance of this interaction for cristae formation remains unclear. As mentioned before, several conflicting conclusions on the function of OPA1 have been published. It is widely accepted that OPA1 is important to maintain the inner membrane architecture but disagreement prevails about the importance of OPA1 for the formation of crista junctions. Thus, it is necessary that the roles of OPA1, especially in its interplay with the MICOS complex, are better characterized. Several studies indicate direct interactions between OPA1, the dimeric $\mathrm{F}_{1} \mathrm{~F}_{\mathrm{O}}$-ATP synthase, and MICOS, suggesting that they cooperate to maintain the inner membrane architecture. Therefore, it is important to dissect the hierarchy of these proteins in cristae formation. 
A

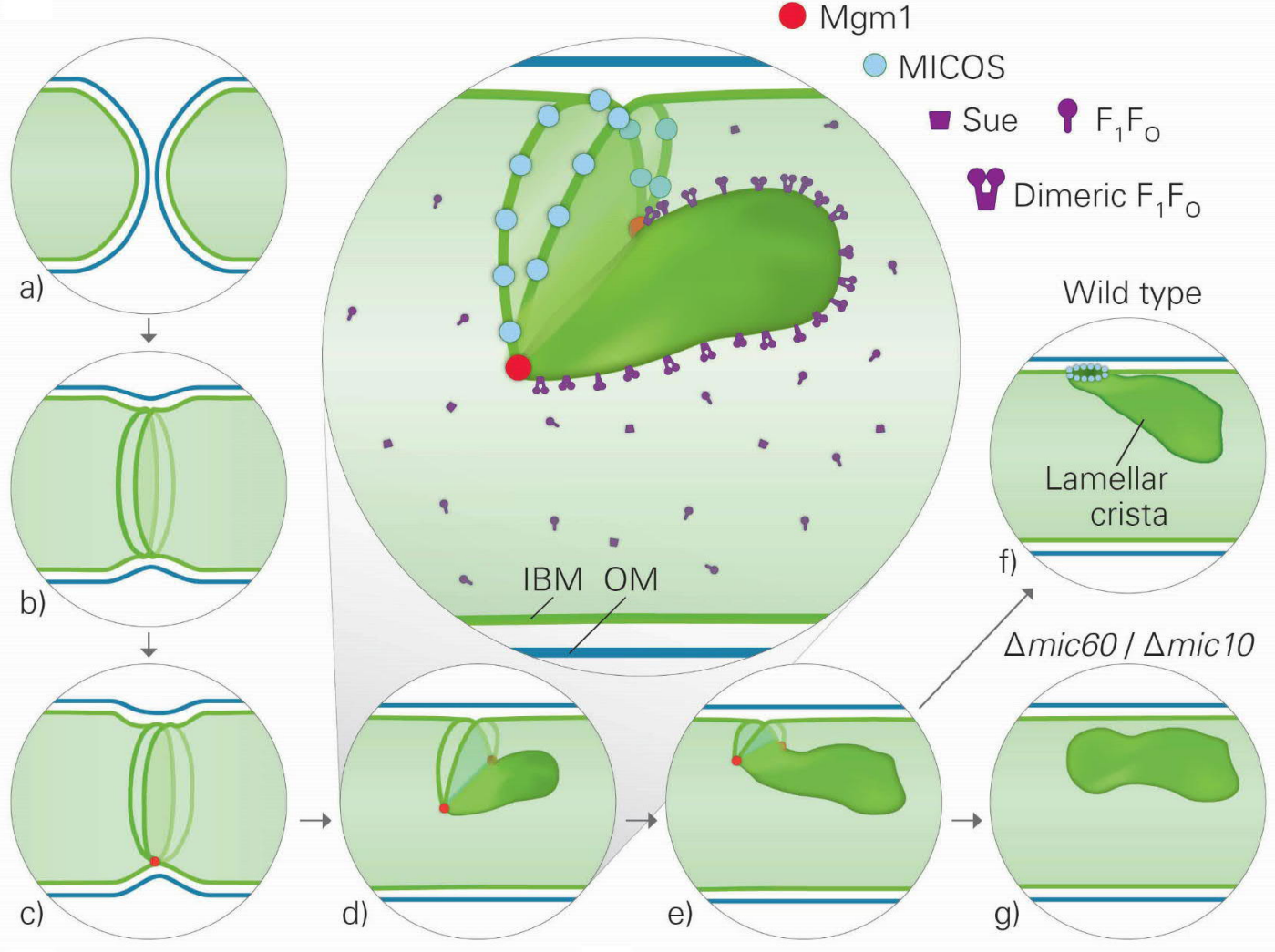

B

C
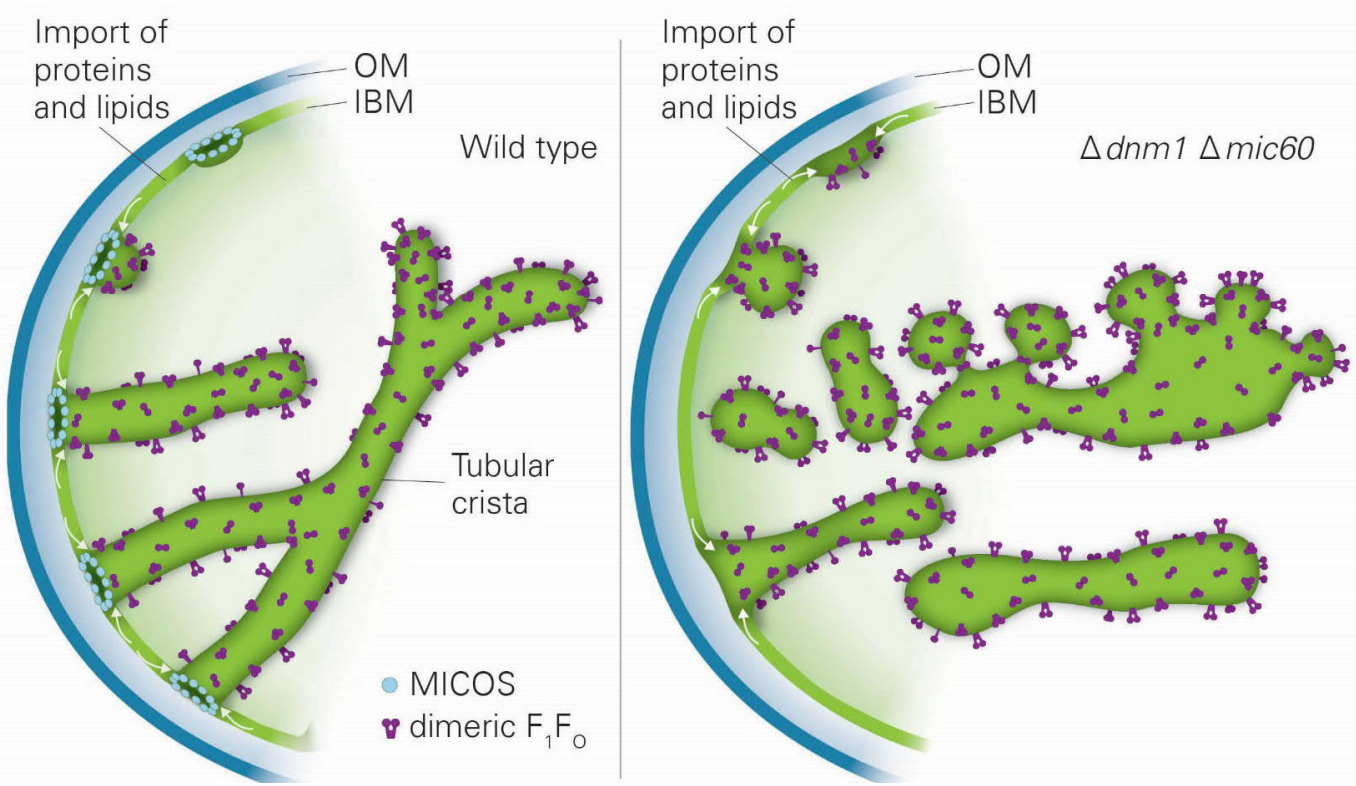

Figure 16: Model of cristae formation in the yeast Saccharomyces cerevisiae. (A-C) The model proposes two distinct pathways of cristae formation. (A) Lamellar cristae are formed by reshaping a fusion-derived septum into a lamellar crista. Membrane remodeling requires MICOS, dimeric $\mathrm{F}_{1} \mathrm{~F}_{\mathrm{O}}$-ATP synthase and Mgm1. Loss of MICOS leads to detachment of lamellae. (B) Tubular cristae are formed in a MICOS-dependent manner and do not require Mgm1. (C) Inhibition of mitochondrial tubule fusion and fission by Dnm1 knockout $(\Delta d n m 1)$ enhances the formation of tubular cristae that detach from the IBM in the absence of the MICOS core subunits Mic10 ( $\triangle$ mic 10 ) or Mic60 ( $\Delta$ mic60). Adapted with permission from: Harner et al., 2016. 


\subsection{Fluorescence Nanoscopy in Mitochondrial Biology}

With the first electron micrographs of mitochondria in the 1950s, electron microscopy has become an essential tool in mitochondrial biology. Due to the development of modern cryoelectron tomography, the method can even provide high-resolution protein structures (Figure 8). However, its restriction to fixed samples prevents the recording of mitochondrial dynamics. In contrast, fluorescence microscopy allows the visualization of mitochondrial dynamics within live cells for prolonged periods. Furthermore, fluorescence microscopy has the advantage of high specificity, as it localizes only specifically labeled targets. Nevertheless, the resolution of conventional fluorescence microscopy is restricted to $\sim 200 \mathrm{~nm}$ due to the diffraction of light. Diffraction-limited fluorescence microscopy therefore does not provide the spatial resolution needed to discern mitochondrial substructures or protein distributions (Jakobs et al., 2020). The development of diffraction-unlimited fluorescence nanoscopy enabled greatly improved resolution down to the nanometer regime even in optical microscopy (Hell and Wichmann, 1994; Klar et al., 2000). In fluorescence nanoscopy, fluorophores are distinguished based on their molecular states to overcome the diffraction barrier. These states are typically a fluorescent "on" state and a non-fluorescent "off" state. Switching between these molecular states allows the majority of fluorophores in a diffraction-limited volume to be rendered dark and for only a subset of molecules to be fluorescent. In coordinate-targeted nanoscopy, a light pattern is used to drive the fluorophores between the two states at defined coordinates in the sample (Sahl et al., 2017). In stimulated emission depletion (STED) nanoscopy, a diffractionlimited excitation beam is coaligned with a second doughnut-shaped laser beam of a longer wavelength and scanned across the specimen. The doughnut-shaped, red-shifted laser beam keeps molecules in the periphery in a non-fluorescent state as it forces their inactivation by stimulated emission. It thereby effectively shrinks the area of fluorescing molecules and improves the spatial resolution (Hell and Wichmann, 1994; Klar et al., 2000). Coordinatestochastic nanoscopy techniques like photo-activated localization microscopy (PALM) or stochastic optical reconstruction microscopy (STORM) (Betzig et al., 2006; Rust et al., 2006), turn on subsets of molecules randomly over time, so that only an individual fluorophore within a distance larger than the diffraction limit fluoresces. By the recording of multiple frames, coordinate-stochastic nanoscopy techniques separate the molecules over extended periods and data reconstruction is necessary to calculate a super-resolved image. STED nanoscopy, however, does not require data reconstruction, which allows relatively fast acquisition of highresolution data and supports its use in live-cell fluorescence nanoscopy (Bottanelli et al., 2016; Sahl et al., 2017).

\subsection{Aims and Scope of the Study}

Mitochondria are dynamic structures that constantly change their morphology (Bereiter-Hahn and Vöth, 1994). It is likely that their inner membrane architecture exhibits similar dynamic 
behavior. However, conventional fluorescence microscopy does not allow for the visualization of cristae. To overcome this hindrance, the first study in this thesis aims to establish STED nanoscopy imaging of cristae in living cells. A major challenge in nanoscopy is the labeling of the target structure as all fluorescence nanoscopy techniques require special labels (Jakobs et al., 2020; Sahl et al., 2017). The combination of self-labeling tags like the SNAP-tag or Halotag (Juillerat et al., 2003) and organic dyes (Lukinavičius et al., 2013) is a promising approach for labeling in live-cell STED nanoscopy. This study aims to adapt this strategy for the analysis of the mitochondrial inner membrane and its dynamics by STED nanoscopy.

The second part of this thesis aims to elucidate the process of cristae formation in human mitochondria. As MICOS is essential for crista junction formation (Laan et al., 2016), the thesis focuses on answering whether the arrangement of MICOS in the inner membrane determines the cristae structure or vice versa. Previous studies in yeast have reported a punctuate distribution of MICOS along mitochondria and have proposed that the MICOS complex determines sites in the inner membrane that facilitate the formation of cristae. However, the respective studies relied on conventional fluorescence microscopy with a resolution close to the diameter of the organelle (Friedman et al., 2015; Hoppins et al., 2011). Therefore, this thesis aims to analyze the submitochondrial localization of the MICOS core subunit Mic60, with respect to the cristae architecture, using diffraction-unlimited STED nanoscopy and 3D electron microscopy.

Various studies have demonstrated that next to MICOS, OPA1 and the dimeric F $F_{1}$-ATP synthase contribute to the formation of proper cristae (Cogliati et al., 2016; Giacomello et al., 2020; Laan et al., 2016). However, many open questions still exist regarding the function of the MICOS subcomplexes and their interplay with OPA1 and the dimeric $\mathrm{F}_{1} \mathrm{~F}_{\mathrm{O}}$-ATP synthase. Moreover, the hierarchy of these proteins in cristae formation is still under debate and thus needs further analysis. As the shape of the cristae differs between organisms and cell lines, data from different cell types are generally difficult to compare, which eventually complicates the analysis of cristae formation (Cogliati et al., 2016; Giacomello et al., 2020; Laan et al., 2016; Rampelt et al., 2017a). To solve this problem, the study aims at a detailed analysis of the function of the aforementioned key players in cristae formation within the same human cell line. To dissect the roles of the individual MICOS subcomplexes, OPA1 and the dimeric $\mathrm{F}_{1} \mathrm{~F}_{\mathrm{O}^{-}}$ ATP synthase, human HeLa cells are depleted of these factors and are analyzed using electron microscopy, super-resolution microscopy, and protein biochemistry. Furthermore, this thesis intends to test the importance of outer membrane fusion and fission for cristae formation in humans. Finally, using the acquired data, the thesis aims to develop a comprehensive model of cristae formation in human mitochondria. 


\section{Chapter 2 - Live-Cell Nanoscopy of Mitochondria}

The following manuscript was published in Scientific Reports

\section{Live-cell STED nanoscopy of mitochondrial cristae}

Till Stephan ${ }^{1,3}$, Axel Rösch ${ }^{1,3}$, Dietmar Riedel ${ }^{2}$ and Stefan Jakobs ${ }^{1,3}$

\section{Author affiliations:}

${ }^{1}$ Department of NanoBiophotonics, Max Planck Institute for Biophysical Chemistry, 37077, Göttingen, Germany. ${ }^{2}$ Laboratory of Electron Microscopy, Max Planck Institute for Biophysical Chemistry, 37077, Göttingen, Germany. ${ }^{3}$ Clinic of Neurology, University Medical Center Göttingen, 37075, Göttingen, Germany.

Correspondence should be addressed to S.J. (email: sjakobs@gwdg.de)

Scientific Reports 9, Article number 12419; DOI: 10.1038/s41598-019-48838-2

Submitted: June 10, 2019; Accepted: August 13, 2019; Published: August 27, 2019 
Chapter 2 - Live-Cell Nanoscopy of Mitochondria

\section{Author contributions:}

T.S. and S.J. conceived the project. T.S. engineered the stable cell line and established live-cell imaging. T.S. and A.R. optimized labeling conditions and recorded STED data. D.R. prepared samples for electron microscopy and recorded electron micrographs. T.S. and A.R., analyzed electron micrographs and STED recordings. T.S. prepared the manuscript and S.J. wrote the paper with comments from all authors. 


\title{
SCIENTIFIC REPORTS
}

natureresearch

\section{Live-cell STED nanoscopy of mitochondrial cristae}

\author{
Till Stephan ${ }^{1,3}$, Axel Roesch ${ }^{1,3}$, Dietmar Riedel ${ }^{2}$ \& Stefan Jakobs $\circledast^{1,3}$ \\ Mitochondria are highly dynamic organelles that exhibit a complex inner architecture. They exhibit \\ a smooth outer membrane and a highly convoluted inner membrane that forms invaginations called \\ cristae. Imaging cristae in living cells poses a formidable challenge for super-resolution light microscopy. \\ Relying on a cell line stably expressing the mitochondrial protein COX8A fused to the SNAP-tag and \\ using STED (stimulated emission depletion) nanoscopy, we demonstrate the visualization of cristae \\ dynamics in cultivated human cells. We show that in human HeLa cells lamellar cristae are often \\ arranged in groups separated by voids that are generally occupied by mitochondrial nucleoids.
}

Mitochondria form tubular and highly dynamic networks in mammalian cells that constantly undergo fusion and fission events ${ }^{1,2}$. They are double-membrane organelles that exhibit a smooth outer membrane and a highly convoluted inner membrane. Cristae are invaginations of the inner membrane that generally adopt tubular or lamellar shapes and project into the matrix space. The cristae architecture adapts to different cellular conditions and is changed upon various stimuli ${ }^{3,4}$. However, the actual cristae dynamics are poorly understood. Electron microscopy (EM) routinely provides the required resolution to visualize cristae, but it is restricted to fixed samples.

The study of cristae in living cells requires light microscopy; a challenge for studying cristae dynamics is the small size of the mitochondria. The diameter of mitochondrial tubules is generally between 200 and $700 \mathrm{~nm}$ and, in many mammalian cell types, the crista-to-crista distance can be below $100 \mathrm{~nm}$. Hence, because of the diffraction limit in optical microscopy ( $250 \mathrm{~nm}$, depending on the wavelength), visualization of individual cristae by light microscopy has been a notorious challenge ${ }^{5}$. The development of various forms of super-resolution microscopy that enable a substantial better or even diffraction-unlimited (nanoscopy) resolution paved the way to overcome this problem. For a detailed discussion of the various techniques see expert reviews ${ }^{6-8}$.

In fixed cells, cristae were first visualized by light microscopy with isoSTED nanoscopy ${ }^{9}$. Using structured illumination microscopy (SIM) providing a resolution of around $100 \mathrm{~nm}$, mitochondrial sub-structures were resolved in living cells ${ }^{10-12}$. However, the discrimination between individual cristae and groups of cristae in human mitochondria remained difficult because of the limited resolution.

Diffraction-unlimited nanoscopy provides a resolution of substantially better than $100 \mathrm{~nm}^{7}$. So far, the overall mitochondrial dynamics and the cristae movements, difficulties in labelling and concerns on light induced photo-toxicity have hampered the visualization of single cristae dynamics using live-cell nanoscopy $7,13,14$

In this study we overcome this problem by using diffraction-unlimited STED super-resolution microscopy (STED nanoscopy) in combination with a genome edited human cell line that enables labeling of the cristae with a silicon rhodamine dye. Thereby, we unequivocally visualize the dynamics of individual cristae in human cells using nanoscopy.

\section{Results}

Visualization of the cristae architecture in living cells. We have generated a human HeLa cell line that stably expresses the full length cytochrome $c$ oxidase subunit 8A (COX8A), an integral protein of the mitochondrial inner membrane, fused to the SNAP-tag ${ }^{15}$. The SNAP-tag is a self-labeling protein that directly reacts with a labeling compound such as a cell permeable fluorophore that is added to the growth medium ${ }^{16}$. Thereby the SNAP-tag provides flexibility in the choice of the fluorophore. As COX8A is a subunit of complex IV of the respiratory chain, it is expected to be preferentially localized in the crista membrane ${ }^{17,18}$. Fusions to COX8A have been used in several studies to highlight mitochondria, without affecting mitochondrial function ${ }^{19}$. The fusion gene was targeted to the chromosomal AAVS1 (adeno-associated virus integration site 1) Safe Harbor Locus

\footnotetext{
${ }^{1}$ Department of NanoBiophotonics, Max Planck Institute for Biophysical Chemistry, 37077, Göttingen, Germany. 'Laboratory of Electron Microscopy, Max Planck Institute for Biophysical Chemistry, 37077, Göttingen, Germany. ${ }^{3}$ Clinic of Neurology, University Medical Center Göttingen, 37075, Göttingen, Germany. Correspondence and requests for materials should be addressed to S.J. (email: sjakobs@gwdg.de)
} 
a

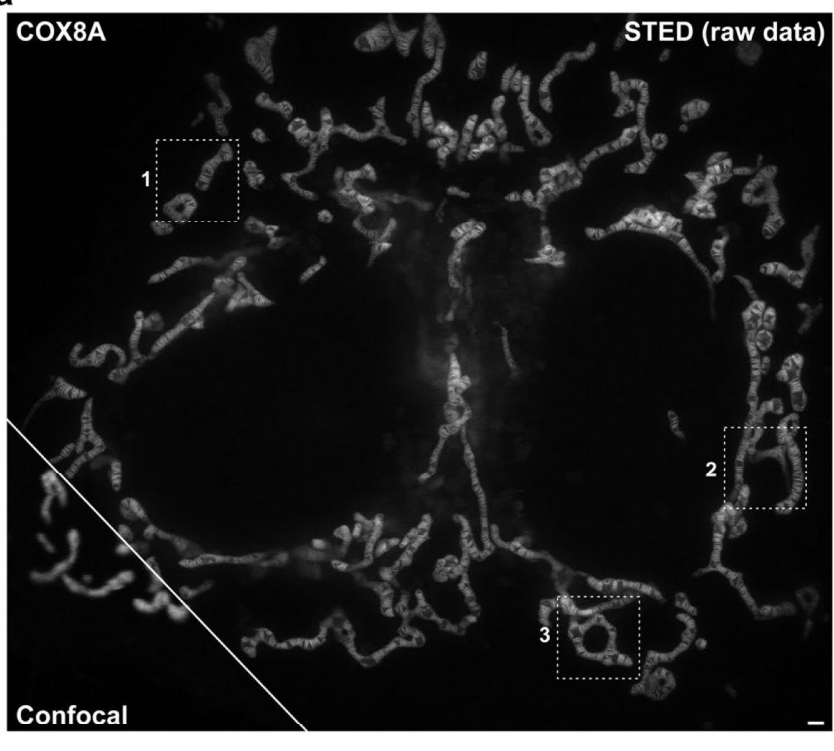

b
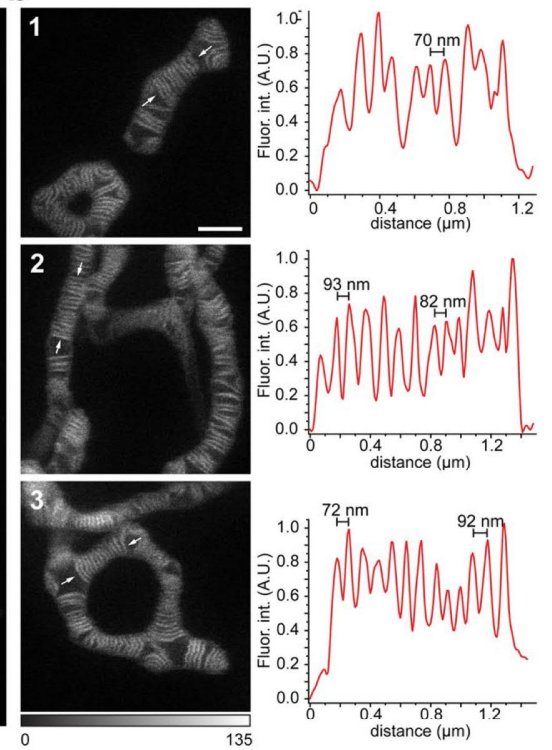

Figure 1. Live-cell Stimulated Emission Depletion (STED) nanoscopy of mitochondrial cristae in HeLa cells. HeLa cells stably expressing COX8A-SNAP fusion proteins were labeled using SNAP-Cell SiR and visualized with STED nanoscopy. (a) Overview of HeLa cells. Shown is a comparison between confocal and STED resolution. (b) Left: Magnifications of the areas indicated in the overview image. Right: Fluorescence intensity line profiles measured as indicated in the magnifications. STED images show unprocessed raw data without background subtraction. Scale bars: $1 \mu \mathrm{m}$.

using a CRISPR/Cas 9 based genome editing strategy, ensuring largely constant expression levels ${ }^{20,21}$. By using this fusion construct we target the SNAP-tag to the mitochondrial inner membrane.

Labeling of living cells by adding the cell-permeant dye SNAP-Cell SiR to the medium ${ }^{22}$ resulted in brightly fluorescent mitochondria (Fig. 1a; Supplementary Fig. S1). As expected, diffraction-limited confocal recordings did not reveal any sub-mitochondrial structures, whereas with STED nanoscopy (excitation: $640 \mathrm{~nm}$, STED: $775 \mathrm{~nm}$ ), we were able to resolve individual cristae in mitochondria of living HeLa cells with $\sim 50 \mathrm{~nm}$ resolution (Fig. 1). We measured the crista-to-crista distances in 15 mitochondria of different living cells, and found a distance of 70 to $100 \mathrm{~nm}$ between closely spaced cristae (Fig. 1b, Supplementary Fig. S2a). Within groups of cristae, the mean crista-to-crista distance was $123 \mathrm{~nm}$ (Supplementary Fig. S2a,c), which is fully in line with the mean crista-to-crista distance of $120 \mathrm{~nm}$ measured on electron micrographs recorded from the same cell line (Fig. 2a, Supplementary Fig. S2b,c). Using live-cell STED nanoscopy, we were able to record entire cells, thereby getting an overview on the cristae architecture of mitochondria with different shapes or with different subcellular positions (Fig. 1).

Mitochondrial nucleoids occupy the voids between groups of cristae. The cristae frequently occurred in groups, separated by voids of several hundred nanometer size. Mitochondrial DNA (mtDNA) is compacted into nucleoprotein complexes termed mitochondrial nucleoids. We used the cell-permeant dye PicoGreen to label the mtDNA and analyzed the location of the mitochondrial nucleoids with respect to the cristae in live mitochondria. Surprisingly, we found that in this cell line most free spaces in the mitochondrial matrix that were devoid of cristae were occupied by mitochondrial nucleoids (Fig. 2b; Supplementary Fig. S3a,b). This finding was fully corroborated by dual-color STED images of chemically fixed HeLa cells labeled with antisera against ATP5I, a subunit of the $\mathrm{F}_{1} \mathrm{~F}_{\mathrm{o}}$-ATP synthase, and against double stranded DNA (Supplementary Fig. S3c).

Time lapse imaging of cristae dynamics. Our labeling strategy allowed us to capture individual cristae and their dynamics across larger fields of view. By this we could, for example, capture the dynamics of cristae apparently moving in groups during the fission of a mitochondrial tubule (Fig. 2c; Supplementary Movie S1). Due to the flexibility of the beam-scanning STED imaging scheme, the size of the recorded field of view can be adapted, facilitating video sequences with frame rates in the second range. Even at this temporal resolution, we observed an unexpected level of cristae movements (Supplementary Movies S2 and S3). When the cells were additionally labeled with the live-cell DNA stain PicoGreen, we could additionally follow the movements of the nucleoids within the mitochondria (Supplementary Movie S4). Due to photobleaching (Supplementary Fig. S3d), the imaging sequences were typically limited to 10-20 frames. We note that after 20 frames the mitochondria generally did not show signs of photostress, which is in line with the observation that STED nanoscopy is live-cell compatible ${ }^{14}$. We envisage that with the development of new dyes that are less prone to photobleaching under live-cell conditions, much longer image sequences should be possible. 
a

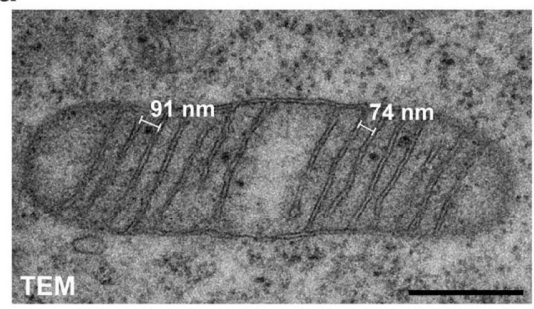

b
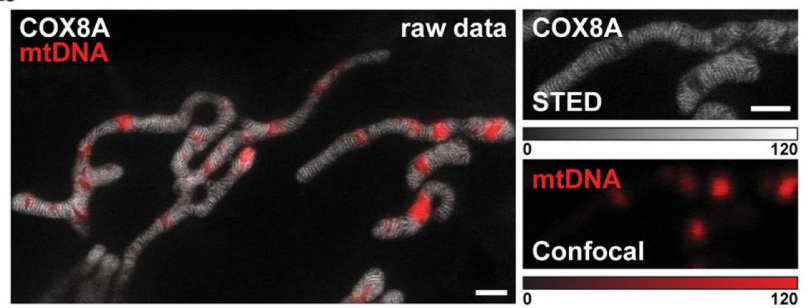

C
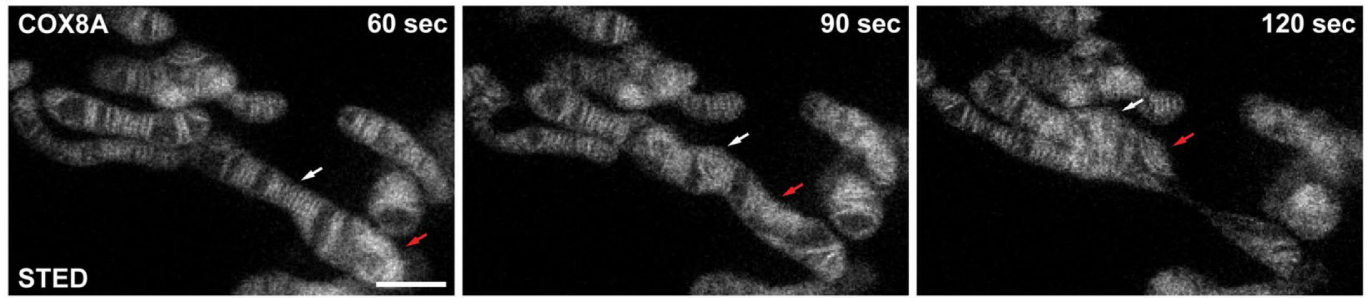

Figure 2. Dynamics of mitochondrial cristae. (a) Transmission electron microscopy (TEM) of mitochondria from HeLa cells. (b) Dual-color live-cell imaging of mitochondria. HeLa cells stably expressing COX8A-SNAP fusion proteins were labeled using SNAP-Cell SiR. In addition, mtDNA was labeled with PicoGreen (red). The cristae were visualized by STED nanoscopy, the nucleoids by confocal scanning light microscopy. (c) Livecell time-lapse STED nanoscopy of mitochondria. Mitochondria were recorded every 15 seconds. Shown are three frames out of ten. Arrows indicate the behavior of two separated groups of cristae during the fission of a mitochondrion. The whole image series is shown in Supplementary Movie S1. (b) Shows unprocessed raw data without background subtraction; in (c) photobleaching was compensated by adapting the color table. Scale bars: a: $0.5 \mu \mathrm{m}$; b,c: $1 \mu \mathrm{m}$.

\section{Discussion}

The intricate mitochondrial membrane architecture is vital for the functioning of mitochondria as cellular powerhouses. It is widely accepted that the mitochondrial cristae are dynamic structures that are remodeled upon various cellular stimuli, but also upon apoptosis and during ageing ${ }^{4,23-25}$. The mechanisms of mitochondrial cristae biogenesis are debated and different mechanisms for this process have been suggested ${ }^{26-28}$. Currently, very little is known about the actual dynamics of mitochondrial cristae in these processes since a clear visualization of the cristae structure in living cells has been a notorious challenge in the past.

To better analyze the formation of cristae and their structure and dynamics under different cellular conditions, a new approach for high-resolution live-cell imaging of mitochondrial cristae is urgently needed. We suggest that the COX8A-SNAP cell line in combination with nanoscopy will be a valuable resource to study cristae dynamics and cristae biogenesis. Our dual-color recordings of cristae and mitochondrial nucleoids revealed that in the cell line we used, nucleoids generally occupy voids between groups of cristae. Mitochondrial nucleoids, because of their low contrast, are usually invisible in conventional EM, although experimentally advanced methods such as electron cryo-tomography ${ }^{29}$ or correlative light-electron microscopy ${ }^{30}$ allowed to visualize nucleoids next to cristae in fixed cells.

We note that our images have been recorded with a commercial STED microscope, which will allow also non-specialized laboratories to record cristae dynamics. STED microscopy might be particularly suitable for the study of cristae dynamics as its scanning approach allows the fast imaging of small fields of view with the possibility to adjust the required optical resolution. When focusing only on a single mitochondrion, the photostress is minimal since the rest of the cell is not illuminated. In this study, we used a STED microscope that improved the resolution in the lateral plane, but was diffraction limited along the $\mathrm{z}$-axis (2D STED nanoscopy). As the HeLa cell line used primarily exhibits lamellar cristae that are oriented perpendicular to the growth surface, this imaging mode was well suited. Other cell types may exhibit tubular or more complex cristae arrangements ${ }^{3,18,28,31}$ and therefore will require 3D nanoscopy in order to provide a full view on the orientation of the cristae in the mitochondria. Clearly, also other nanoscopies including RESOLFT/non-linear SIM ${ }^{32-34}$ are likely to be able to visualize individual cristae dynamics. As diffraction-limited linear SIM, enabling a resolution of $100 \mathrm{~nm}$, has been previously used to visualize cristae or groups of cristae in living cells ${ }^{10-12}$, it will be interesting to compare the different imaging modalities on the same cell type.

While this paper was in review, another study reported on the development of the fluorescent probe MitoPB Yellow, which labels mitochondrial cristae and facilitates their visualization using STED nanoscopy ${ }^{35}$. As MitoPB Yellow binds to membranes, its use does not rely on the expression of fusion proteins, but provides no freedom in the choice of the wavelengths used for imaging. The SNAP-tag that we used to tag COX8A allows to change the fluorophore according to the requirements of the different light microscopy techniques. This versatility will facilitate an immediate comparison of different fluorophores, labelling strategies and imaging modalities on a dynamic and challenging live-cell sample. Moreover, the chemical fixation of the double membraned mitochondria is often difficult and we expect this cell line will be a valuable resource for evaluating and systematically improving fixation conditions for optical microscopy. 


\section{Material and Methods}

Cloning of plasmids. To generate the donor plasmid AAVS1-Blasticidin-CAG-COX8A-SNAP, the plasmid AAVS1-Basticidin-CAG-Flpe-ERT2 was first linearized by using the restriction endonucleases SalI and EcoRV. AAVS1-Blasticidin-CAG-Flpe-ERT2 was a gift from Su-Chun Zhang (Addgene plasmid \#68461; http://n2t.net/ addgene:68461; RRID:Addgene_68461).

COX8A-SNAP was amplified by PCR from pSNAPf-COX8A (New England Biolabs, Ipswich, MA, USA) using the primers given below and subsequently integrated into the linearized plasmid by Gibson assembly.

Primers for donor Plasmid:

\section{COX8A-fwd: TCTCATCATTTTGGCAAAGAATTCGTCGACGCCGCCACCATGTCCGTCCTGACGCCG COX8A-rev: GAGGTTGATTATCGATAAGCTTGATATCTTAATTAACCTCGAGTTTAAACGCGGATC}

The gRNA plasmid PX458-AAVS1 was derived from PX458. pSpCas9(BB)-2A-GFP (PX458) was a gift from Feng Zhang (Addgene plasmid \#48138; http://n2t.net/addgene:48138; RRID:Addgene_48138). In brief, oligonucleotides were annealed by primer annealing and integrated into PX458 after linearization with the BbsI restriction endonuclease.

Oligonucleotides for gRNA plasmid:

\section{AAVS1-gRNA-fw: CACCGTGTCCCTAGTGGCCCCACTG AAVS1-gRNA-rev: AACCAGTGGGGCCACTAGGGACAC}

Cell culture. HeLa cells were cultured in DMEM containing $4.5 \mathrm{~g} / \mathrm{L}$ glucose and GlutaMAX ${ }^{\mathrm{TM}}$ additive (Thermo Fisher Scientific) supplemented with $100 \mathrm{U} / \mathrm{mL}$ penicillin and $100 \mu \mathrm{g} / \mathrm{mL}$ streptomycin (Merck Millipore, Burlington, MA, USA), $1 \mathrm{mM}$ sodium pyruvate (Sigma Aldrich) and $10 \%$ (v/v) fetal bovine serum (Merck Millipore).

Generation of a stable cell line. To generate the stable cell line, HeLa cells were co-transfected with the plasmids AAVS1-Blasticidin-CAG-COX8A-SNAP and PX458-AAVS1 using the jetPRIME transfection reagent (Polyplus, Illkirch, France). Starting two days after transfection, the cells were selected using DMEM containing $10 \mu \mathrm{g} / \mathrm{mL}$ Blasticidin S (Invivogen, Toulouse, France) for 7 days. Two weeks after transfection, the cells were stained with DMEM containing $1 \mu$ M SNAP-Cell SiR (New England Biolabs, Ipswich, MA, USA) for about 10 minutes. After two washing steps with DMEM ( $15 \mathrm{~min})$, the cells were detached. Using fluorescence-activated cell sorting (FACS), single fluorescent cells were transferred into 96 well plates. After about 3 weeks, single cell clones were again stained using the SNAP-cell SiR dye and clonal cell lines with labeled mitochondria were selected. Expression of the COX8A-SNAP fusion protein was verified by Western blotting.

Staining of live cells for STED nanoscopy. Cells were seeded in glass bottom dishes (ibidi GmbH, Martinsried, Germany) one day before the measurements. Cells were stained with DMEM containing $1 \mu M$ SNAP-Cell SiR (New England Biolabs) and optionally 0.1\% (v/v) Quant-IT PicoGreen dsDNA reagent (Thermo Fisher Scientific, Waltham, MA, USA) $\left(15 \mathrm{~min}, 37^{\circ} \mathrm{C}\right)$. After removing the staining solution and two washing steps with DMEM, the cells were left in the incubator in DMEM for 15-30 minutes to remove unbound dye. Prior to imaging, DMEM was replaced with life cell imaging solution (Thermo Fisher Scientific).

Staining of fixed cells for STED nanoscopy. Cells were seeded on coverslips and fixed with pre-warmed $\left(37^{\circ} \mathrm{C}\right) 8 \%$ formaldehyde in phosphate buffered saline for $7 \mathrm{~min}$ (PBS, $137 \mathrm{mM} \mathrm{NaCl}, 2.68 \mathrm{mM} \mathrm{KCl}$ and $10 \mathrm{mM}$ $\mathrm{Na}_{2} \mathrm{HPO}_{4}, \mathrm{pH}$ 7.4). The cells were afterwards permeabilized with $0.5 \%$ Triton X-100 in PBS (5 min) and blocked with a solution of $5 \%(\mathrm{w} / \mathrm{v})$ bovine serum albumin (BSA) in PBS (10 min, RT). Cells were labeled for ATP5I and double-stranded DNA with specific antisera (Proteintech, Rosemont, IL, USA, Abcam, Cambridge, UK). Primary antibodies were diluted in $5 \%$ BSA (w/v) in PBS and added to the samples ( $1 \mathrm{~h}, \mathrm{RT})$. Samples were washed several times with PBS and blocked with BSA solution $(5 \mathrm{~min}, \mathrm{RT})$. The primary antibodies were detected with secondary anti-mouse antibodies labeled with Alexa Fluor 594 (Thermo Fisher Scientific, Waltham, MA, USA) or anti-rabbit antibodies (Jackson Immuno Research Laboratories, West Grove, PA, USA) custom labeled with the dye Abberior STAR RED (Abberior, Göttingen, Germany) ( $1 \mathrm{~h}, \mathrm{RT})$. The samples were washed five times with PBS and mounted in Mowiol mounting medium containing $0.1 \%(\mathrm{w} / \mathrm{v})$ DABCO (Sigma Aldrich, St. Louis, MO, USA).

STED nanoscopy. STED nanoscopy was performed using a quad scanning STED microscope (Abberior Instruments, Göttingen, Germany) equipped with a UPlanSApo 100x/1,40 Oil objective (Olympus, Tokyo, Japan). The pinhole was set to 0.7-1.0 Airy units. A pixel size of $20-25 \mathrm{~nm}$ was used.

For live STED imaging, SNAP-Cell SiR was excited at $640 \mathrm{~nm}$ and STED was performed at $775 \mathrm{~nm}$ wavelength. PicoGreen was excited at $485 \mathrm{~nm}$ wavelength. The fluorescence signal was detected using avalanche photo diodes with bandpass filters. For STED imaging of SNAP-Cell SiR, a gating of $0.75-8 \mathrm{~ns}$ was applied. Dwell times of 7-10 $\mu$ s were used. For STED images, each line was scanned 4 to 8 times and the signal was accumulated. For the confocal images, each line was scanned once.

For dual color STED imaging of fixed cells, STAR RED was excited at $640 \mathrm{~nm}$ and STED was performed at $775 \mathrm{~nm}$ wavelength. AlexaFluor 594 was excited at $561 \mathrm{~nm}$ wavelength. The fluorescence signal was detected using avalanche photo diodes with bandpass filters and a gating of $0.75-8 \mathrm{~ns}$ was applied. Dwell times of $10 \mu \mathrm{s}$ were used. Each line was scanned 3 times and the signal was accumulated. 
Image processing. No deconvolution was used. All main still images (Figs 1a,b and $2 \mathrm{~b}$ ) display unprocessed raw data without background subtraction. For time lapse recordings (Fig. 2c; Supplementary Movies S1-S4), photobleaching was compensated using the Bleach Correction function (histogram matching) in the Fiji software. For dual-color live-cell recordings, background subtraction was performed (5\%-10\% of the maximum signal).

Transmission EM of HeLa cells. HeLa cells were grown on aclar discs (Plano, Wetzlar, Germany) to a confluency of about $60 \%$ and fixed with pre-warmed $2.5 \%$ glutaraldehyde in $0.1 \mathrm{M}$ cacodylate buffer $(\mathrm{pH} 7.4,1 \mathrm{~h}$, RT). Samples were stored in the fixative over night to complete fixation $\left(4^{\circ} \mathrm{C}\right)$. Cells were washed three times with $0.1 \mathrm{M}$ cacodylate buffer and were stained in $1 \%(\mathrm{w} / \mathrm{v})$ osmium tetroxide in $0.1 \mathrm{M}$ cacodylate buffer $(\mathrm{pH} 7.4,1 \mathrm{~h}$, $\mathrm{RT}$ ). The samples were washed with distilled water (three times, each for $5 \mathrm{~min}$ ) and stained en-bloc for $30 \mathrm{~min}$ in aqueous $1 \%(\mathrm{w} / \mathrm{v})$ uranyl acetate (RT, in the dark). Dehydration was performed using an ethanol series of 30, 50,70 and $100 \%$ (three times, each for $5 \mathrm{~min}$ ) with a final dehydration step in propylene oxide $(5 \mathrm{~min})$. Cells were embedded in Agar 100 epoxy resin. Sections of $70 \mathrm{~nm}$ thickness were recorded on a Philips CM120 transmission electron microscope with a TVIPS $2 \mathrm{k} \times 2 \mathrm{k}$ slow-scan CCD camera.

\section{Cell line availability}

The COX8A-SNAP-tag cell line is available from the corresponding author for non-commercial use upon reasonable request.

\section{Data Availability}

All raw data used to create the figures and videos in this paper are available from the corresponding author upon reasonable request.

\section{References}

1. Bereiter-Hahn, J. \& Voth, M. Dynamics of mitochondria in living cells: shape changes, dislocations, fusion, and fission of mitochondria. Micros Resarch \& Tech 27, 198-219 (1994).

2. Friedman, J. R. \& Nunnari, J. Mitochondrial form and function. Nature 505, 335-343 (2014).

3. Scheffler, I. E. Mitochondria, second edition. John Wiley \& Sons, Inc. Hoboken, New Jersey, USA (2008).

4. Cogliati, S., Enriquez, J. A. \& Scorrano, L. Mitochondrial Cristae: Where Beauty Meets Functionality. Trends Biochem Sci 41, 261-273 (2016)

5. Jakobs, S. \& Wurm, C. A. Super-resolution microscopy of mitochondria. Curr Opin Chem Biol 20, 9-15 (2014).

6. Ji, N., Shroff, H., Zhong, H. \& Betzig, E. Advances in the speed and resolution of light microscopy. Curr Opin Neurobiol 18, 605-616 (2008).

7. Sahl, S. J., Hell, S. W. \& Jakobs, S. Fluorescence nanoscopy in cell biology. Nat Rev Mol Cell Biol 18, 685-701 (2017).

8. Sigal, Y. M., Zhou, R. \& Zhuang, X. Visualizing and discovering cellular structures with super-resolution microscopy. Science 361, $880-887$ (2018)

9. Schmidt, R. et al. Mitochondrial Cristae Revealed with Focused Light. Nano Letters 9, 2508-2510 (2009).

10. Shao, L., Kner, P., Rego, E. H. \& Gustafsson, M. G. Super-resolution 3D microscopy of live whole cells using structured illumination. Nat Methods 8, 1044-1046 (2011).

11. Fiolka, R., Shao, L., Rego, E. H., Davidson, M. W. \& Gustafsson, M. G. Time-lapse two-color 3D imaging of live cells with doubled resolution using structured illumination. Proc Natl Acad Sci USA 109, 5311-5315 (2012).

12. Huang, X. et al. Fast, long-term, super-resolution imaging with Hessian structured illumination microscopy. Nat Biotechnol 36, 451-459 (2018).

13. Wäldchen, S., Lehmann, J., Klein, T., van de Linde, S. \& Sauer, M. Light-induced cell damage in live-cell super-resolution microscopy. Sci Rep 5, 15348 (2015).

14. Kilian, N. et al. Assessing photodamage in live-cell STED microscopy. Nat Methods 15, 755-756 (2018).

15. Keppler, A. et al. A general method for the covalent labeling of fusion proteins with small molecules in vivo. Nat Biotechnol. 21, 86-89 (2003).

16. Hinner, M. J. \& Johnsson, K. How to obtain labeled proteins and what to do with them. Curr Opin Biotechnol 21, 766-776 (2010).

17. Gilkerson, R. W., Selker, J. M. L. \& Capaldi, R. A. The cristal membrane of mitochondria is the principal site of oxidative phosphorylation. FEBS Lett 546, 355-358 (2003).

18. Stoldt, S. et al. Spatial orchestration of mitochondrial translation and OXPHOS complex assembly. Nat Cell Biol 20, 528-534 (2018).

19. Liu, X., Yang, L., Long, Q., Weaver, D. \& Hajnoczky, G. Choosing proper fluorescent dyes, proteins, and imaging techniques to study mitochondrial dynamics in mammalian cells. Biophys Rep 3, 64-72 (2017).

20. Hockemeyer, D. et al. Efficient targeting of expressed and silent genes in human ESCs and iPSCs using zinc-finger nucleases. Nat Biotechnol 27, 851-857 (2009).

21. DeKelver, R. C. et al. Functional genomics, proteomics, and regulatory DNA analysis in isogenic settings using zinc finger nucleasedriven transgenesis into a safe harbor locus in the human genome. Genome Res 20, 1133-1142 (2010).

22. Lukinavicius, G. et al. A near-infrared fluorophore for live-cell super-resolution microscopy of cellular proteins. Nat Chem $\mathbf{5}$, 132-139 (2013).

23. Daum, B., Walter, A., Horst, A., Osiewacz, H. D. \& Kuhlbrandt, W. Age-dependent dissociation of ATP synthase dimers and loss of inner-membrane cristae in mitochondria. Proc Natl Acad Sci USA 110, 15301-15306 (2013).

24. Brandt, T. et al. Changes of mitochondrial ultrastructure and function during ageing in mice and Drosophila. Elife 6, https://doi. org/10.7554/eLife.24662 (2017).

25. McArthur, K. et al. BAK/BAX macropores facilitate mitochondrial herniation and mtDNA efflux during apoptosis. Science 359, https://doi.org/10.1126/science.aao6047 (2018).

26. Zick, M., Rabl, R. \& Reichert, A. S. Cristae formation-linking ultrastructure and function of mitochondria. Biochim Biophys Acta 1793, 5-19(2009).

27. Harner, M. E. et al. An evidence based hypothesis on the existence of two pathways of mitochondrial crista formation. Elife 5, https:// doi.org/10.7554/eLife.18853 (2016).

28. Blum, T. B., Hahn, A., Meier, T., Davies, K. M. \& Kuhlbrandt, W. Dimers of mitochondrial ATP synthase induce membrane curvature and self-assemble into rows. Proc Natl Acad Sci USA 116, 4250-4255 (2019).

29. Kukat, C. et al. Cross-strand binding of TFAM to a single mtDNA molecule forms the mitochondrial nucleoid. Proc Natl Acad Sci USA 112, 11288-11293 (2015).

30. Kopek, B. G., Shtengel, G., Xu, C. S., Clayton, D. A. \& Hess, H. F. Correlative 3D superresolution fluorescence and electron microscopy reveal the relationship of mitochondrial nucleoids to membranes. Proc Natl Acad Sci USA 109, 6136-6141 (2012). 
31. Stoldt, S. et al. Mic60 exhibits a coordinated clustered distribution along and across yeast and mammalian mitochondria. Proc Natl Acad Sci USA 116, 9853-9858 (2019).

32. Grotjohann, T. et al. Diffraction-unlimited all-optical imaging and writing with a photochromic GFP. Nature 478, 204-208 (2011).

33. Brakemann, T. et al. A reversibly photoswitchable GFP-like protein with fluorescence excitation decoupled from switching. Nat Biotechnol 29, 942-947 (2011).

34. Li, D. et al. Extended-resolution structured illumination imaging of endocytic and cytoskeletal dynamics. Science 349, https://doi. org/10.1126/science.aab3500 (2015).

35. Wang, C. et al. A photostable fluorescent marker for the superresolution live imaging of the dynamic structure of the mitochondrial cristae. Proc Natl Acad Sci USA 116, 15817-15822 (2019).

\section{Acknowledgements}

We thank Jaydev Jethwa for carefully reading the manuscript and Peter Ilgen for help with graphics. This work was supported by the Deutsche Forschungsgemeinschaft (DFG, German Research Foundation) funded SFB1286 (project A05) and under Germany's Excellence Strategy - EXC 2067/1- 390729940 (both to SJ).

\section{Author Contributions}

T.S. and S.J. conceived the project; T.S., A.R. and D.R. performed research; T.S. and A.R. analyzed data. S.J. wrote the text with comments from all authors.

\section{Additional Information}

Supplementary information accompanies this paper at https://doi.org/10.1038/s41598-019-48838-2.

Competing Interests: The authors declare no competing interests.

Publisher's note: Springer Nature remains neutral with regard to jurisdictional claims in published maps and institutional affiliations.

(c) (i) Open Access This article is licensed under a Creative Commons Attribution 4.0 International License, which permits use, sharing, adaptation, distribution and reproduction in any medium or format, as long as you give appropriate credit to the original author(s) and the source, provide a link to the Creative Commons license, and indicate if changes were made. The images or other third party material in this article are included in the article's Creative Commons license, unless indicated otherwise in a credit line to the material. If material is not included in the article's Creative Commons license and your intended use is not permitted by statutory regulation or exceeds the permitted use, you will need to obtain permission directly from the copyright holder. To view a copy of this license, visit http://creativecommons.org/licenses/by/4.0/.

(c) The Author(s) 2019 


\section{Supplementary Information for}

\section{Live-cell STED nanoscopy of mitochondrial cristae}

Till Stephan ${ }^{\mathrm{a}, \mathrm{c}}$, Axel Roesch $^{\mathrm{a}, \mathrm{c}}$, Dietmar Riedel ${ }^{\mathrm{b}}$, Stefan Jakobs ${ }^{\mathrm{a}, \mathrm{c}}$

${ }^{a}$ Department of NanoBiophotonics, Max Planck Institute for Biophysical Chemistry, 37077

Göttingen, Germany; ${ }^{b}$ Laboratory of Electron Microscopy, Max Planck Institute for Biophysical Chemistry, 37077 Göttingen, Germany; ${ }^{\mathrm{c}}$ Clinic of Neurology, University Medical Center Göttingen, 37075 Göttingen, Germany

Correspondence to SJ (sjakobs@gwdg.de)

This PDF file includes:

Supplementary Figures S1-S3

Supplementary Figure Legends

Captions for Supplementary Movies S1-S4

Other supplementary materials for this manuscript include the following:

Supplementary Movies S1-S4 


\section{Supplementary Figure S1}
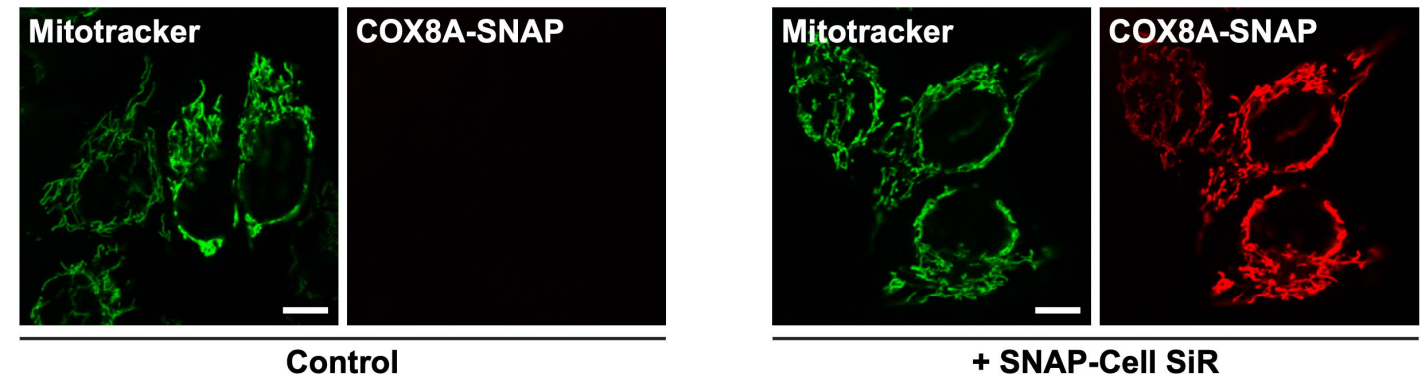


\section{Supplementary Figure S1}

Specificity of SNAP-labeling. HeLa cells stably expressing COX8A-SNAP fusion proteins were imaged without (Control, left) and after addition of the SNAP-Cell SiR dye to the growth medium (right). The mitochondria were highlighted with the mitochondria-specific dye Mitotracker Green. Scale bar: $10 \mu \mathrm{m}$. 
a
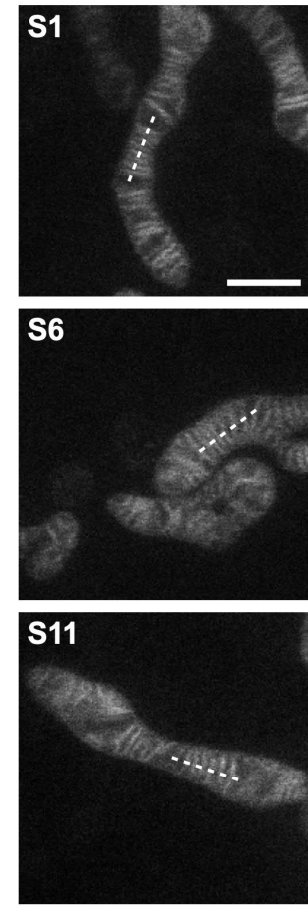

b
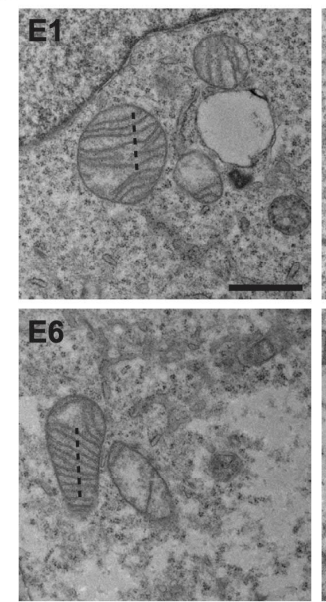

C

\begin{tabular}{|c|c|c|c|c|c|c|c|}
\hline $\begin{array}{l}\text { STED } \\
\text { image }\end{array}$ & $\begin{array}{l}\text { mean crista } \\
\text { to crista } \\
\text { distance } \\
\text { in } \mathrm{nm}\end{array}$ & & SD & $\begin{array}{c}\text { EM } \\
\text { image }\end{array}$ & $\begin{array}{l}\text { mean crista } \\
\text { to crista } \\
\text { distance } \\
\text { in nm }\end{array}$ & & SD \\
\hline S1 & 100 & \pm & 22 & E1 & 139 & \pm & 18 \\
\hline S2 & 111 & \pm & 60 & E2 & 108 & \pm & 21 \\
\hline S3 & 155 & \pm & 72 & E3 & 127 & \pm & 40 \\
\hline S4 & 156 & \pm & 71 & E4 & 118 & \pm & 18 \\
\hline S5 & 113 & \pm & 28 & E5 & 113 & \pm & 23 \\
\hline S6 & 134 & \pm & 49 & E6 & 118 & \pm & 20 \\
\hline s7 & 110 & \pm & 32 & E7 & 110 & \pm & 26 \\
\hline & 100 & $\begin{array}{l} \pm \\
\pm\end{array}$ & 20 & E8 & 152 & $\begin{array}{l} \pm \\
\pm\end{array}$ & 33 \\
\hline s9 & 147 & \pm & 70 & E9 & 132 & \pm & 32 \\
\hline s10 & 107 & \pm & 16 & E10 & 87 & & 17 \\
\hline s11 & 124 & \pm & 38 & & & & \\
\hline s12 & 140 & \pm & 59 & & & & \\
\hline S13 & 134 & \pm & 50 & & & & \\
\hline S14 & $\begin{array}{l}104 \\
123\end{array}$ & \pm & 20 & & & & \\
\hline S15 & 88 & \pm & 17 & & & & \\
\hline mean & $123 \mathrm{~nm}$ & & & mean & $120 \mathrm{~nm}$ & & \\
\hline
\end{tabular}
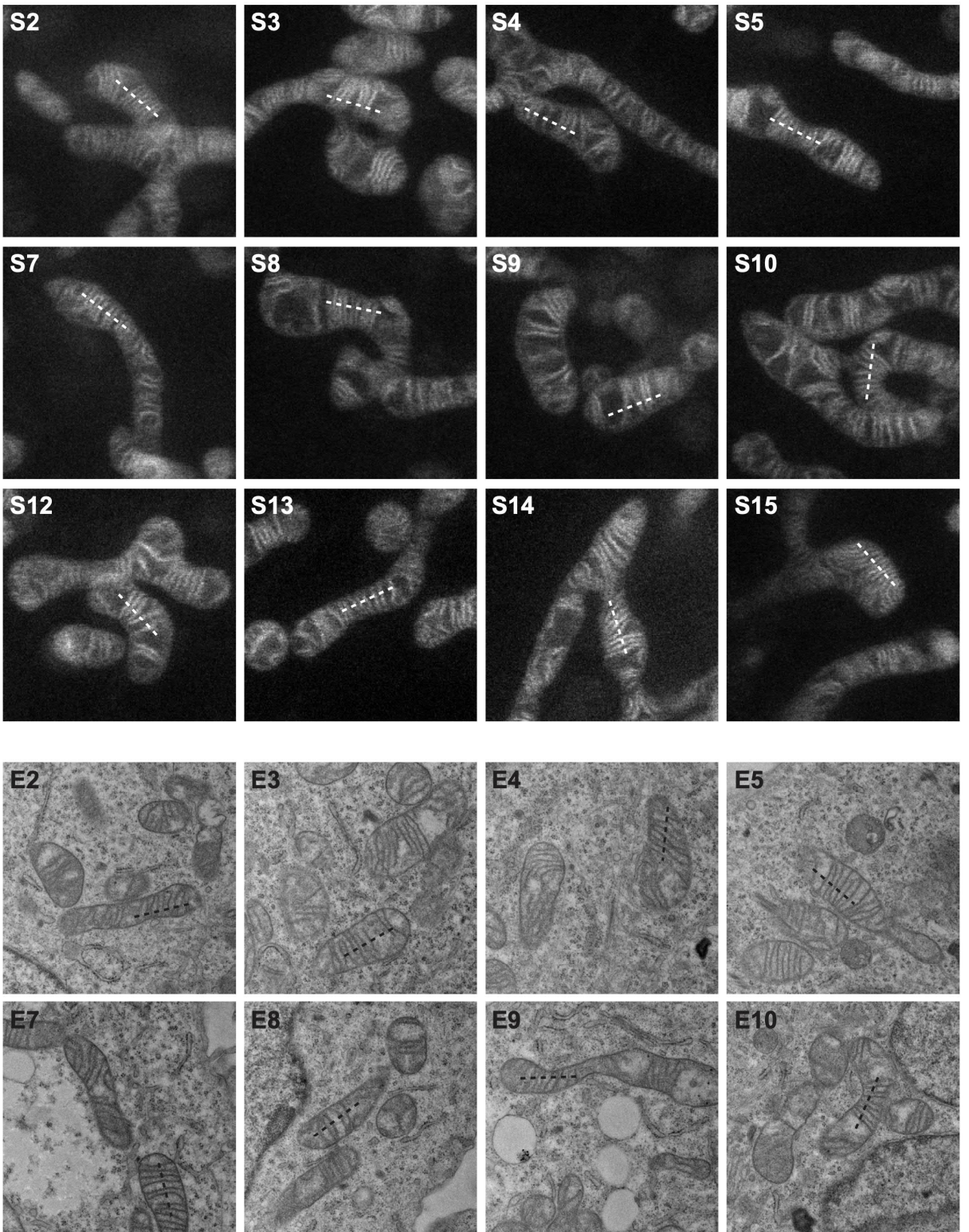

$120 \mathrm{~nm}$

\section{Supplementary Figure S2}




\section{Supplementary Figure S2}

Quantification of crista-to-crista distances in mitochondria. a. Determination of crista-to-crista distances on live-cell STED images. HeLa cells stably expressing COX8A-SNAP were recorded by STED nanoscopy. On 15 manually selected mitochondria from nine different cells the fluorescence intensity was measured by line profiles (indicated by dashed lines, each line profile is $1 \mu \mathrm{m}$ long). The crista-to-crista distances within the respective crista groups were estimated based on these measurements. b. Determination of crista-to-crista distances on transmission electron microscopy images. On manually selected mitochondria the crista-to-crista distances were estimated by line profiles (indicated by dashed lines, each line profile is $1 \mu \mathrm{m}$ long). Electron micrographs were randomly selected from a total number of 93 images randomly taken from at least 10 different cells. c. Table of mean crista-to-crista distances on mitochondria determined on STED nanoscopy and EM images. The table shows the mean crista-to-crista distance for every line profile shown in (a) and (b). SD: Standard deviation. Scale bars: $1 \mu \mathrm{m}$. 


\section{Supplementary Figure S3}

a

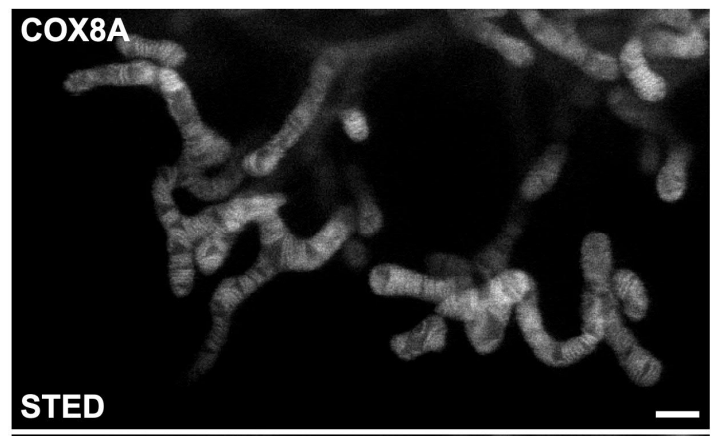

mtDNA

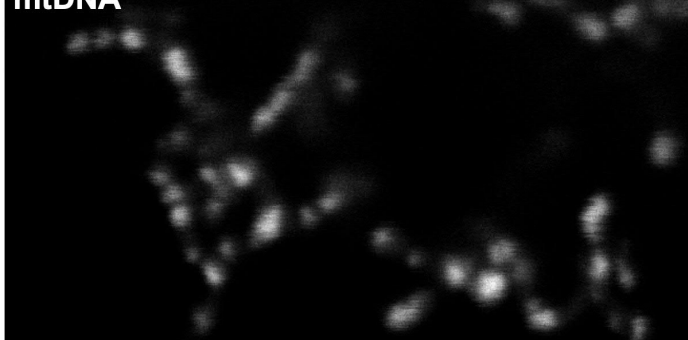

confocal

merged

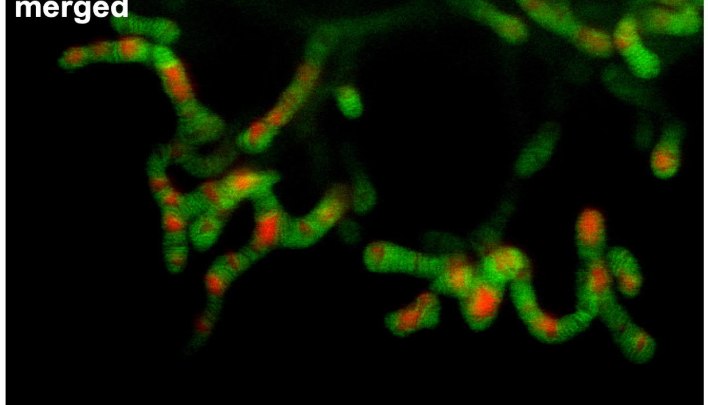

C

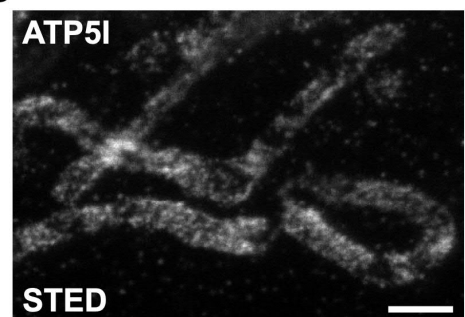

d

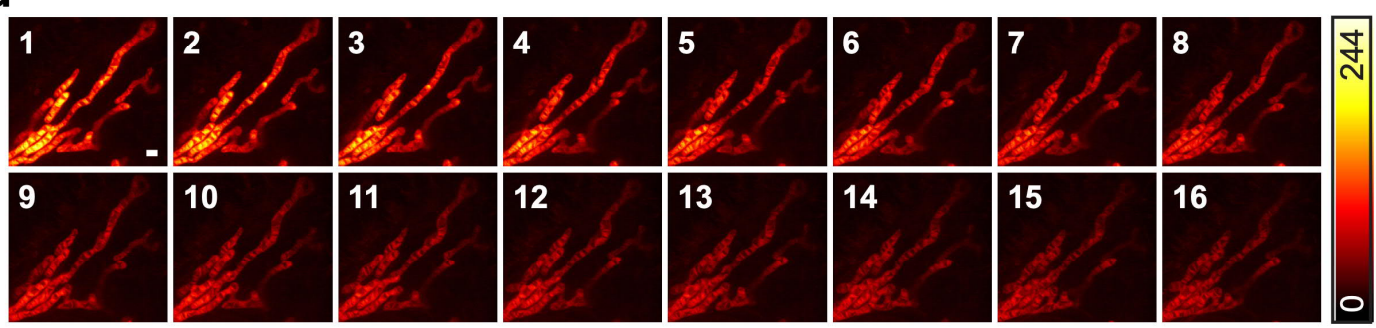

b
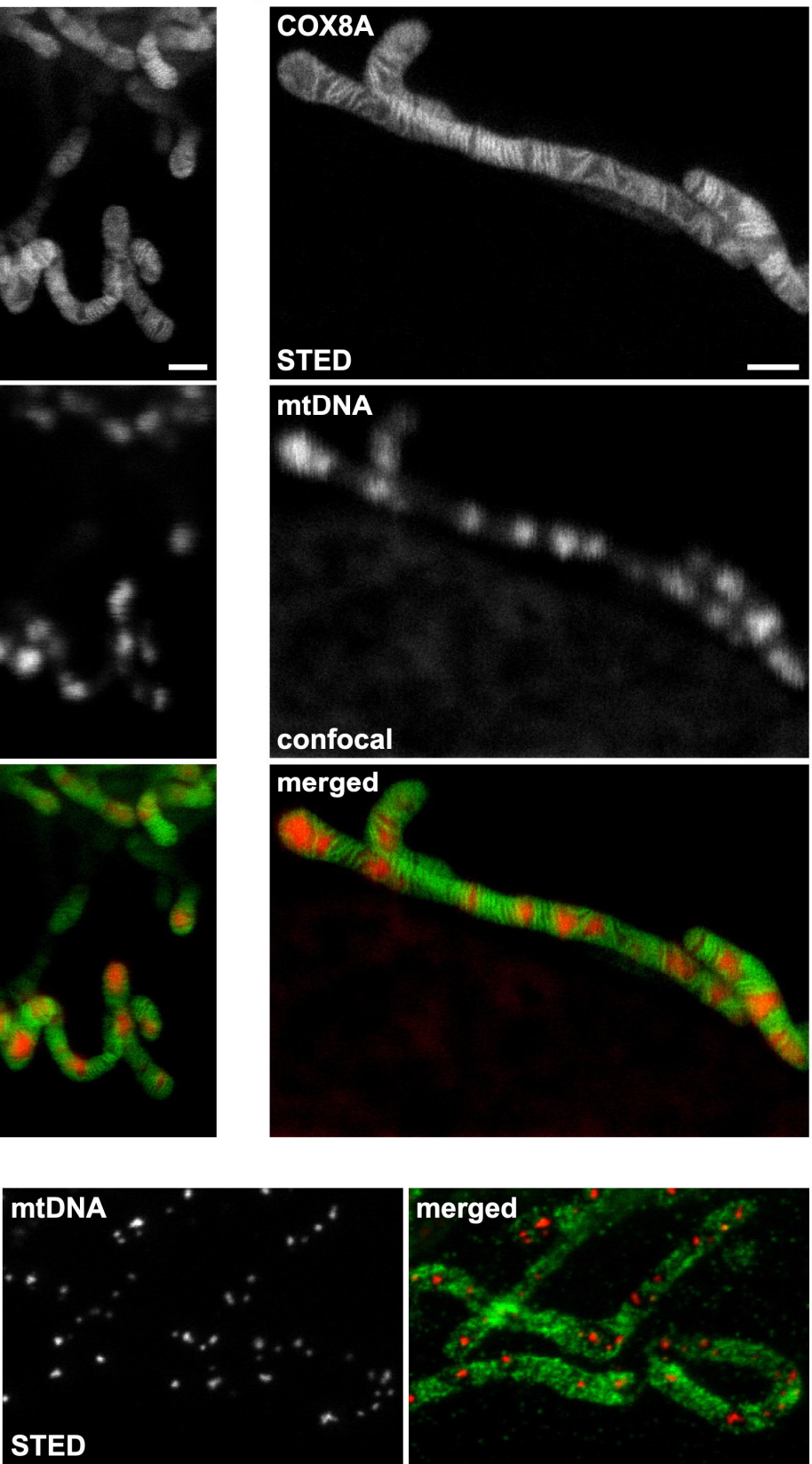


\section{Supplementary Figure S3}

STED nanoscopy. a, b. Live-cell dual-color recordings of the mitochondrial inner membrane and mtDNA. HeLa cells stably expressing COX8A-SNAP were labeled with SNAP-Cell SiR to highlight the mitochondrial inner membrane and with PicoGreen to label the mtDNA. SNAP-Cell SiR was recorded by STED nanoscopy and PicoGreen in the confocal mode. Shown are representative images from two different cells. c. STED nanoscopy of mtDNA and ATP5I in chemically fixed HeLa cells. HeLa cells were immunolabeled with antisera specific for double stranded DNA and against ATP5I, a subunit of the $\mathrm{F}_{1} \mathrm{~F}_{0}$-ATP synthase. Cells were recorded with dual-color STED nanoscopy. d. Photobleaching in live-cell STED nanoscopy. HeLa cells stably expressing COX8A-SNAP were labeled with SNAP-Cell SiR and recorded by STED nanoscopy. Shown are the raw data images from an image series of 16 consecutive live-cell STED recordings. Recording time for one frame: 15 seconds. The same color coding is used for all images to demonstrate the amount of photobleaching. The same image data are shown in Supplementary Movie S4, but with an adjustment of the color coding to correct for photobleaching. In a-c, but not in $\mathrm{d}$, we performed a background subtraction (5\%-10\% of the maximum signal). Scale bars: $1 \mu \mathrm{m}$. 


\section{Supplementary Movie S1 to S3}

Live-cell time-lapse STED nanoscopy of mitochondria. HeLa cells stably expressing COX8ASNAP fusion proteins were labeled using SNAP-Cell SiR and visualized with STED nanoscopy. Mitochondria were recorded every 15 (Movie S1), 10 (Movie S2) or 5 (Movie S3) seconds. Scale bar: $1 \mu \mathrm{m}$. The movies show raw data; photobleaching was compensated by adapting the color table. Scale bar: $1 \mu \mathrm{m}$.

\section{Supplementary Movie S4}

Dual-color live-cell microscopy of mitochondria. Hela cells stably expressing COX8A-SNAP fusion proteins were labeled with SNAP-Cell SiR and PicoGreen (to visualize mtDNA). Mitochondria were recorded every 15 seconds. COX8A-SNAP (grey) was recorded in STED mode, PicoGreen (red) in the confocal mode. Photobleaching was compensated by adapting the color table; to remove background, we subtracted $5 \%$ of the maximal signal. Scale bar: $1 \mu \mathrm{m}$. 
Chapter 2 - Live-Cell Nanoscopy of Mitochondria

The supplementary movies included in this manuscript can be accessed on the following websites:

Supplementary Movie S1

Supplementary Movie S2

Supplementary Movie S3

Supplementary Movie S4 


\section{Chapter 3 - Mic60 Distribution in Mitochondria}

The following manuscript was published in Proceedings of the National Academy of Sciences of the United States of America (PNAS)

\section{Mic60 exhibits a coordinated clustered distribution along and across yeast and mammalian mitochondria}

Stefan Stoldt ${ }^{1, *}$, Till Stephan ${ }^{1,}$, Daniel C. Jans ${ }^{2,3}$, Christian Brüser ${ }^{1}$, Felix Lange ${ }^{1}$, Jan KellerFindeisen ${ }^{1}$, Dietmar Riedel ${ }^{4}$, Stefan W. Hell ${ }^{1}$ and Stefan Jakobs ${ }^{1,2}$

\section{Author affiliations:}

${ }^{1}$ Department of NanoBiophotonics, Max Planck Institute for Biophysical Chemistry, 37077 Göttingen, Germany. ${ }^{2}$ Clinic of Neurology, University Medical Center Göttingen, 37075 Göttingen, Germany. ${ }^{3}$ Science for Life Laboratory, Department of Applied Physics, Royal Institute of Technology, 17165 Stockholm, Sweden. ${ }^{4}$ Laboratory of Electron Microscopy, Max Planck Institute for Biophysical Chemistry, 37077 Göttingen, Germany.

*S.S. and T.S. contributed equally to this work.

Correspondence should be addressed to S.W.H. or S.J (email: stefan.hell@mpibpc.mpg.de; sjakobs@gwdg.de).

PNAS 116 (20), 9853-9858. DOI: 10.1073/pnas.1820364116

Submitted: November 29, 2018; Accepted: March 14, 2019; Published: April 26, 2019 


\section{Author contributions}

S.S., T.S and S.J. conceived the project and designed the experiments. S.S. produced yeast strains and prepared yeast samples for STED nanoscopy. S.S. and T.S. optimized immunolabeling for STED nanoscopy. S.S., T.S. and C.B. performed 2D STED nanoscopy of yeast cells and human cells. D.C.J. recorded 3D STED nanoscopy data. D.R., F.L., S.S. and T.S. prepared samples for electron microscopy. F.L. recorded TEM data. S.S., T.S., C.B., J.K.F., S.J. and S.W.H. analyzed STED data. T.S. analyzed TEM data. S.S. and T.S. analyzed and segmented FIB-SEM data. T.S. created 3D models of FIB-SEM data. S.S. and T.S. prepared the manuscript and wrote the paper together with S.J. 


\title{
Mic60 exhibits a coordinated clustered distribution along and across yeast and mammalian mitochondria
}

\author{
Stefan Stoldt ${ }^{\mathrm{a}, 1}$, Till Stephan ${ }^{\mathrm{a}, 1}$, Daniel C. Jans ${ }^{\mathrm{b}, \mathrm{c}}$, Christian Brüser ${ }^{\mathrm{a}}$, Felix Lange ${ }^{\mathrm{a}}$, Jan Keller-Findeisen ${ }^{\mathrm{a}}$, Dietmar Riedel ${ }^{\mathrm{d}}$,
} Stefan W. Hell ${ }^{a, 2}$, and Stefan Jakobs ${ }^{a, b, 2}$

aDepartment of NanoBiophotonics, Max Planck Institute for Biophysical Chemistry, 37077 Göttingen, Germany; ${ }^{b}$ Clinic of Neurology, University Medical Center Göttingen, 37075 Göttingen, Germany; 'Science for Life Laboratory, Department of Applied Physics, Royal Institute of Technology, 17165 Stockholm, Sweden; and Laboratory of Electron Microscopy, Max Planck Institute for Biophysical Chemistry, 37077 Göttingen, Germany

Contributed by Stefan W. Hell, March 14, 2019 (sent for review November 29, 2018; reviewed by Bo Huang and Nikolaus Pfanner)

Mitochondria are tubular double-membrane organelles essential for eukaryotic life. They form extended networks and exhibit an intricate inner membrane architecture. The MICOS (mitochondrial contact site and cristae organizing system) complex, crucial for proper architecture of the mitochondrial inner membrane, is localized primarily at crista junctions. Harnessing superresolution fluorescence microscopy, we demonstrate that Mic60, a subunit of the MICOS complex, as well as several of its interaction partners are arranged into intricate patterns in human and yeast mitochondria, suggesting an ordered distribution of the crista junctions. We show that Mic60 forms clusters that are preferentially localized in the inner membrane at two opposing sides of the mitochondrial tubules so that they form extended opposing distribution bands. These Mic60 distribution bands can be twisted, resulting in a helical arrangement. Focused ion beam milling-scanning electron microscopy showed that in yeast the twisting of the opposing distribution bands is echoed by the folding of the inner membrane. We show that establishment of the Mic60 distribution bands is largely independent of the cristae morphology. We suggest that Mic60 is part of an extended multiprotein interaction network that scaffolds mitochondria.

mitochondria | MICOS | mitoskeleton | FIB-SEM | nanoscopy

M ochondria, the "powerhouses" of the cell, are doublemembrane organelles that often form highly dynamic loose networks of tubules that frequently undergo fusion and fission, thereby continually changing the appearance of the network (1-3). Mitochondria communicate with the nucleus (4) and are physically connected to other organelles by means of interorganellar tethering $(5,6)$. The highly convoluted mitochondrial inner membrane is surrounded by the smooth outer membrane and has three contiguous domains: the inner boundary membrane that parallels the outer membrane; crista membranes, invaginations of the inner membrane that differ in shape depending on the cell type and physiological conditions; and crista junctions, narrow tubules that connect the inner boundary membrane with the cristae membrane.

Mitochondria arose from the engulfment of a proteobacterium by a precursor of the modern eukaryotic cell (7). Most, if not all, bacterial cells contain at least one skeletal protein system, often referred to as the "prokaryotic cytoskeleton" $(8,9)$. These prokaryotic cytoskeletons are vital for several functions, including cell shape determination and cell division. However, while in eukaryotic cells the cytoskeleton is defined by constitutive components, such as actin, tubulin, and intermediate filaments, the term prokaryotic cytoskeleton is generally used in a broader sense, encompassing a variety of systems that form superstructures within the bacterial cells $(8,9)$. Surprisingly, given the intricate architecture of the mitochondria, a large scaffolding structure, or "mitoskeleton," has not yet been demonstrated.

The mitochondrial contact site and cristae organizing system (MICOS; previously also known as MINOS, MitOs, or MIB) (10) is a conserved ancient hetero-oligomeric protein complex localized primarily at the crista junctions. The depletion of MICOS subunits results in a disturbed inner membrane morphology and a decreased number of crista junctions (11-15). The MICOS core component, Mic60, interacts physically with numerous proteins in the mitochondrial inner and outer membrane, thereby forming contact sites $(12,13,15-20)$.

An earlier study insightfully proposed that MICOS forms a filamentous structure within the inner membrane of budding yeast mitochondria (13). This study relied on genetic interactions and diffraction-limited fluorescence microscopy, providing an optical resolution in the size regimen of the organelle diameter. Here, using stimulated emission depletion (STED) nanoscopy and focused ion beam milling combined with scanning electron microscopy (FIB-SEM), we analyzed the localization of the MICOS complex in relation to the inner membrane architecture. We show that subunits of the yeast and human MICOS complex form distinct clusters that are intricately arranged within the mitochondrial inner membrane, often localized in opposing distribution bands that can adopt a helical arrangement. Our data support the idea that MICOS is part of a multiprotein interaction network that encompasses MICOS as well as additional proteins in the mitochondrial membranes and thereby scaffolds the mitochondrion. We suggest that this scaffold is the elusive mitoskeleton.

\section{Significance}

The intricate folding of the mitochondrial inner membrane is essential for the functioning of mitochondria as cellular powerhouses. The mitochondrial contact site and cristae organizing system (MICOS) complex is localized at the crista junctions and is key for the establishment of proper architecture of the mitochondrial inner membrane. Relying on optical nanoscopy and focused ion beam-scanning electron microscopy, we demonstrate that Mic60, a subunit of the MICOS complex, forms clusters distributed in two opposing distribution bands, which can be twisted to produce a helical arrangement of the cristae. Our findings suggest that the Mic60 clusters are physically coupled along and across the mitochondrial tubules. The visualization of these distributions bands opens the door to a microscopic investigation of the proteins that scaffold mitochondria.

Author contributions: S.S., T.S., and S.J. designed research; S.S., T.S., D.C.J., C.B., F.L., and D.R. performed research; J.K.-F. contributed new reagents/analytic tools; S.S., T.S., D.C.J., C.B., J.K.-F., S.W.H., and S.J. analyzed data; and S.S., T.S., and S.J. wrote the paper.

Reviewers: B.H., University of California, San Francisco; and N.P., University of Freiburg. The authors declare no conflict of interest.

This open access article is distributed under Creative Commons Attribution-NonCommercialNoDerivatives License 4.0 (CC BY-NC-ND).

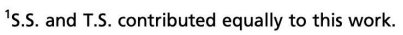

${ }^{2}$ To whom correspondence may be addressed. Email: stefan.hell@mpibpc.mpg.de or sjakobs@gwdg.de.

This article contains supporting information online at www.pnas.org/lookup/suppl/doi:10. 1073/pnas.1820364116/-/DCSupplemental.

Published online April 26, 2019 


\section{Results}

Crista Junctions Are Frequently Arranged in a Helical Pattern in Budding Yeast Mitochondria. The MICOS core component Mic60 is an integral protein of the mitochondrial inner membrane required for the proper folding of this membrane $(10,11)$. To reevaluate the nanoscale distribution of Mic60 in the budding yeast Saccharomyces cerevisiae, we genomically tagged Mic60 with GFP and immunolabeled the cells with a GFP antibody, thereby carefully optimizing the labeling conditions. Because of the small diameter of the mitochondria, diffraction-limited confocal microscopy was insufficient to reveal details of the submitochondrial distribution of Mic60 (Fig. 1A). Using STED nanoscopy $(21,22)$, we found that Mic60 was frequently located in distinct clusters on two opposing sides of a mitochondrion, so that they appeared to be enriched in two opposing distribution bands that were running along the mitochondrial tubules (Fig. $1 A$ and $B$ and SI Appendix, Fig. S1 $A$ ). This clustered distribution could not be attributed to a fixation or imaging artifact, as STED recordings of yeast cells expressing matrix-targeted GFP showed a homogenous distribution of the fluorescence signal across the mitochondria with no indications of clusters (SI Appendix, Fig. S1B). In more than $45 \%$ of all cells $(n=303)$, sections of the Mic60 distribution bands appeared to be twisted, so that the arrangement of the Mic60 clusters was reminiscent of a helix

As Mic60 is enriched at crista junctions $(11,12,23)$, such a helical arrangement suggests that the crista junctions and also the cristae are preferentially arranged in a spiral pattern. To test this hypothesis, we performed FIB-SEM and created 3D reconstructions of the inner membrane architecture of the mitochondrial network of wild-type yeast cells (Fig. 1C, SI Appendix, Fig. S1 $C$ and $D$, and Movie $\mathrm{S} 1$ ). The $3 \mathrm{D}$ reconstructions revealed that most cristae were rather sheet-like, and that almost none of them spanned the mitochondria from one side to the other. The generally rather short cristae were found mostly at two opposing sides of the mitochondrial tubule (SI Appendix, Fig. S1 $E-G$ ). In large parts of the mitochondria, a spiral-like arrangement of the cristae was visible (Fig. $1 C$ and $D, S I$ Appendix, Fig. S $1 E-G$, and Movie S1). Intriguingly, the animated display of virtual consecutive transversal sections along a mitochondrion resulted in the impression of a propeller-like rotation of the cristae (Fig. $1 D$ and $E$ and Movie S1). Even without 3D reconstructions, this rotation of the cristae was immediately visible in the majority $(>80 \%)$ of all yeast cells within a FIB-SEM stack (Fig. $1 F$ ). Thus, the STED data and FIB-SEM recordings point to an ordered arrangement of Mic60, the cristae, and the crista junctions in yeast mitochondria.

Although we observed helical Mic60 patterns in approximately $45 \%(n=303)$ of the yeast cells, a detailed quantification beyond the repeated observation of the helical arrangement of Mic60 clusters (SI Appendix, Fig. S1A) is very challenging in yeast, because this analysis is inevitably hampered by the strong bending of the mitochondrial tubules within the small spherical cells. Furthermore, immunolabeling of yeast cells for superresolution
A

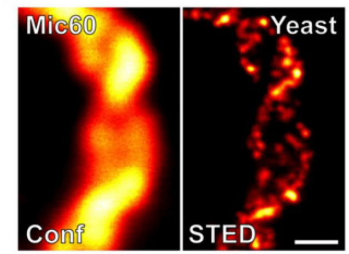

B

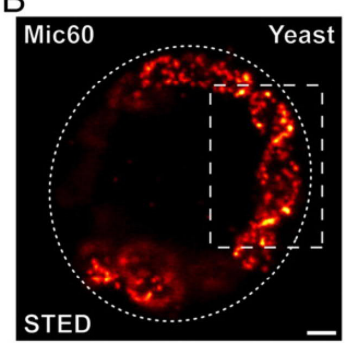

section 2
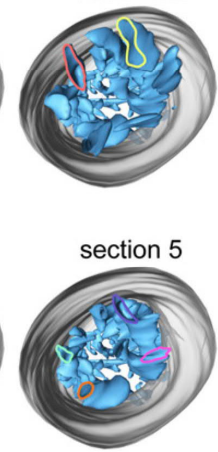

C
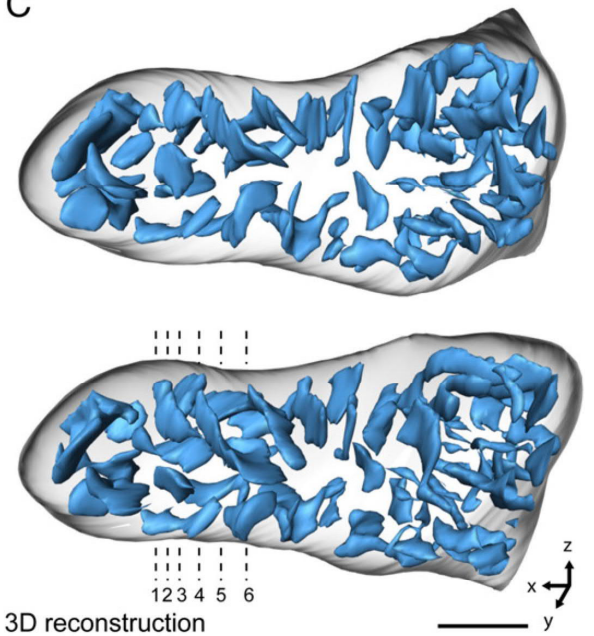

E sections 1-6
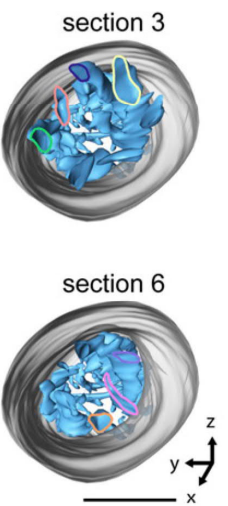

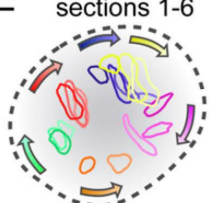

projection

$\mathrm{F}$

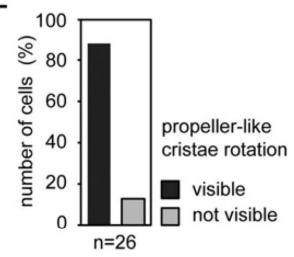

Fig. 1. Mic60 and the cristae are often arranged in a helical pattern in budding yeast mitochondria. $(A$ and $B$ ) Submitochondrial localization of Mic60-GFP in budding yeast grown in galactose medium. Cells were labeled with a GFP-specific antiserum. $(A)$ The comparison between confocal microscopy and STED nanoscopy demonstrates the resolution improvement achieved by STED nanoscopy. (B) 2D STED image of an entire yeast cell; the image shows the same cell as magnified in $A$, as indicated by a rectangle. (C) FIBSEM recording of a budding yeast cell grown in galactose medium. Part of a reconstructed mitochondrion is shown. The cristae are rendered in blue; the outer membrane, in gray. Shown are two different views of the same mitochondrion. (D) Consecutive transversal cross-sections through the reconstructed mitochondrion at sites indicated in C. The contours of six cristae are highlighted with different colors to visualize the orientation of the cristae along the longitudinal axis of the mitochondrion. $(E)$ Projections of gitudinal axis of the mitochondrion. $(E)$ Projections of
the cristae contours shown in $C$. Colors fade with ascending section numbers. Arrows indicate the apparent rotation of the cristae. $(F)$ Quantification of yeast cells showing twisted cristae arrangements, based on FIB-SEM recordings. (Scale bars: $400 \mathrm{~nm}$ in $A$ and $B$; $150 \mathrm{~nm}$ in $C$ and $D$.) 
A

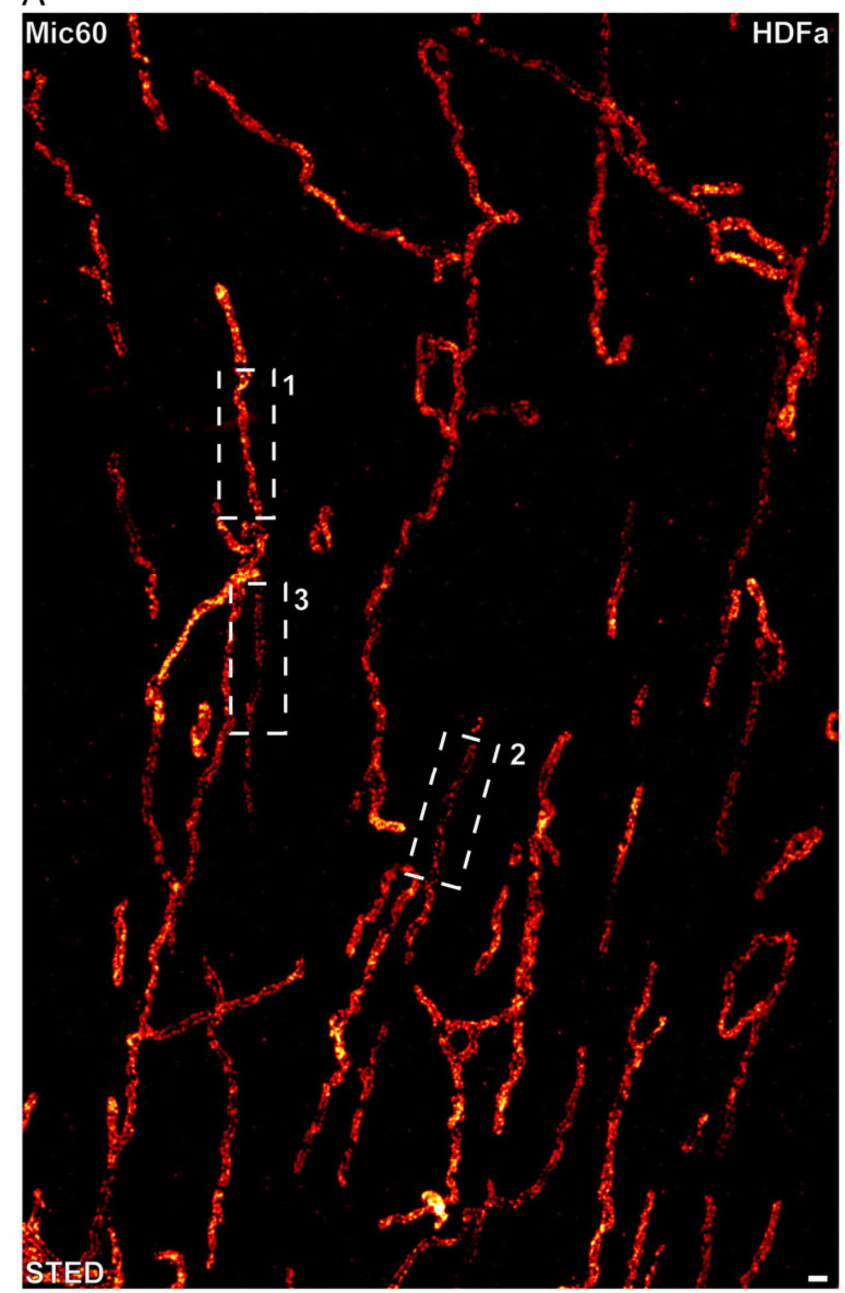

$\mathrm{C}$

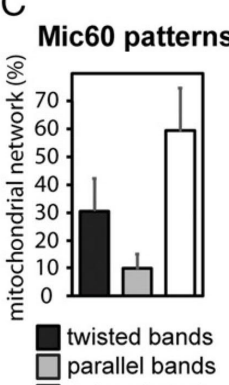

D

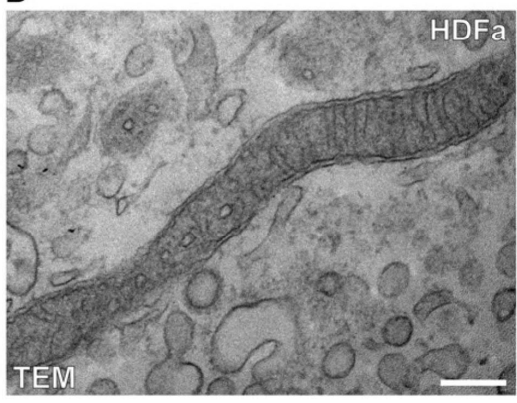

B
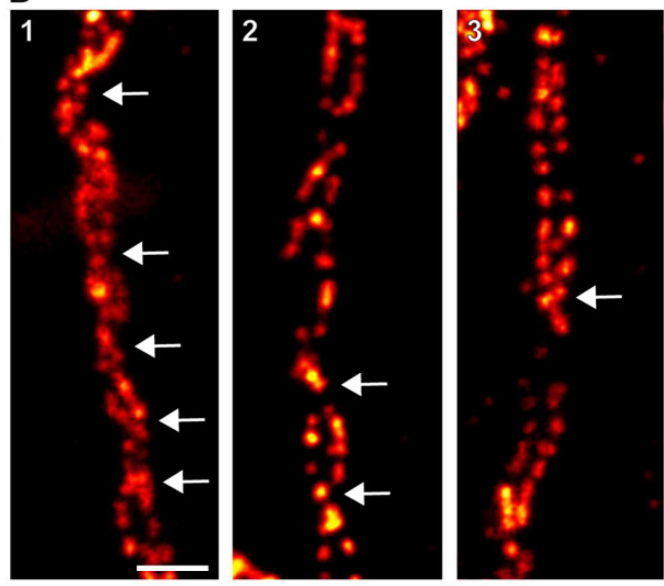

E
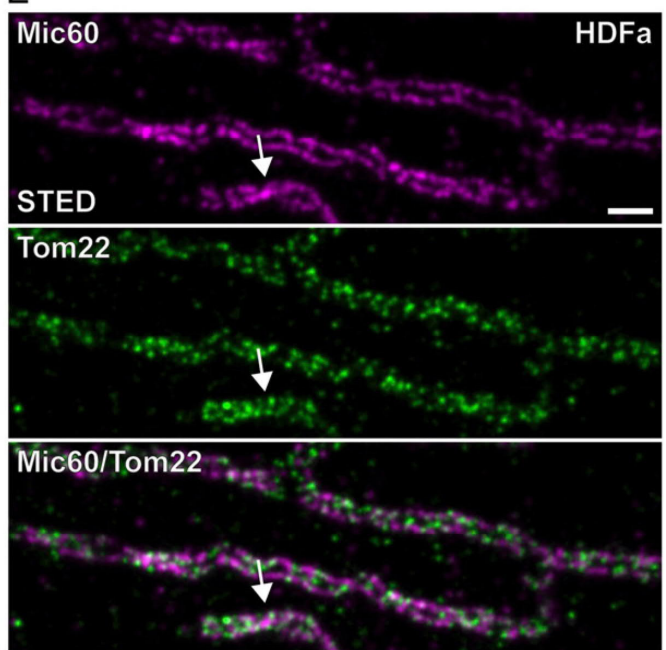

$\mathrm{F}$

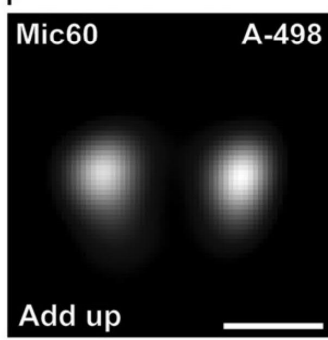

A-498

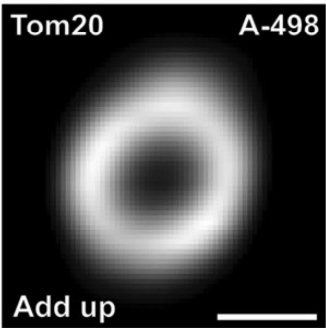

Fig. 2. Nanoscale distribution of Mic60 in human mitochondria. (A) STED nanoscopy of Mic60 in primary HDFa cells. (B) Magnifications of the areas indicated in A. Arrows indicate twisting sites. (C) Quantification of the frequency of occurrences of twisted or parallel Mic60 distribution bands in HDFa cells. For the analysis, parts of the mitochondrial networks of $18 \mathrm{HDFa}$ cells, corresponding to $\sim 14-\mathrm{mm}$ mitochondrial tubules, were manually assigned by three test persons to the different categories. Error bars represent SDs of the three independent analyses. (D) TEM image of a mitochondrion of an HDFa cell. (E) Dual-color STED image of an HDFa cell decorated for Mic60 and Tom22. The arrow points to a twisting site of the Mic60 distribution bands, whereas the outer membrane labeled by Tom 22 is not constricted. $(F)$ Longitudinal add ups of Mic60 and Tom 20 fluorescence signals in mitochondria from human A-498 cells. The add ups cover 1.5- $\mu \mathrm{m}$ sections of the mitochondrial tubule. For Mic60, a tubule section with a parallel distribution of Mic60 clusters was selected. Shown are exemplary images; statistical analysis data are provided in SI Appendix, Fig. S3. For all images, Mic60, Tom22, and Tom20 were decorated with specific antisera. (Scale bars: $500 \mathrm{~nm}$ in $A, B$, and $E ; 200 \mathrm{~nm}$ in $C$ and $F$.) 
microscopy is technically demanding, resulting in numerous damaged cells, and thus requires a manual selection of the imaged cells

A Helical Arrangement of Mic60 Clusters Is Also Frequent in Human Mitochondria. We next investigated whether the helical arrangement of the Mic60 clusters is a peculiarity of the highly curved yeast mitochondria or whether it can also be found in human mitochondria. We decorated adult human dermal fibroblasts (HDFa) with an antiserum against Mic60 and recorded them with 2D STED nanoscopy (Fig. $2 A$ and $B$ and SI Appendix, Fig. $\mathrm{S} 2 A$ ). As reported previously (23), the MICOS clusters were spaced regularly along the longitudinal axis of mitochondria and often appeared to be localized in two parallel distribution bands in the mitochondrial tubule. Manual assignment of large areas of the mitochondrial network revealed that in HDFa cells, these bands of clusters were parallel in approximately $10 \%$ of the mitochondrial network, while twists of the Mic60 distribution bands were observed in approximately $30 \%$ of the mitochondrial network (Fig. $2 C$ and SI Appendix, Fig. S2A). The remaining $\sim 60 \%$ of the network was not clearly assignable to one type of pattern, because of either partially out-of-focus mitochondria or an excessively high density of Mic60 clusters. Possibly the distribution bands were also wider in these cases, so that they were no longer clearly discernible. Parallel and helical arrangements of Mic60 distribution bands were found in the same cell, often in immediate proximity (Fig. $2 A$ and $B$ and SI Appendix, Fig. S2A), demonstrating that the twisted arrangement is not restricted to curved mitochondria.

To investigate the mean distance between the individual Mic60 clusters, we used a computer algorithm to straighten in silico 91 mitochondrial tubules from 13 fibroblasts (SI Appendix, Fig. S2B). The mean distance between neighboring clusters within one distribution band along the longitudinal axis of the mitochondria was $135 \pm 72 \mathrm{~nm}$ (SI Appendix, Fig. S2 B and C). The clusters in one distribution band seemed to be spatially correlated with the clusters in the opposing band. To quantify this observation, we determined the correlation of the Mic60 signal of directly opposing sides in the straightened mitochondria. The Pearson correlation coefficient was 0.39 between the two bands. When one of the distribution bands was shifted in silico with respect to the other by $>500 \mathrm{~nm}$, the Pearson correlation coefficient was almost 0 , which indicates no correlation. From this, we conclude that compared with a random scenario, that Mic60 clusters often appear in opposing pairs.

Using transmission EM (TEM), we next analyzed the cristae architecture in these primary fibroblasts. The cristae exhibited heterogeneous forms, ranging from tubular to lamellar (Fig. $2 D$ and SI Appendix, Fig. S2D). The mean distance between neighboring crista junctions as measured on TEM sections from 10 independent fibroblasts was $100 \pm 34 \mathrm{~nm}$. This spacing is within the same range as seen for the distance of Mic60 clusters (SI Appendix, Fig. S2 $C$ and $E$ ).

Dual-color STED images of mitochondria labeled for the outer membrane protein Tom 22 and Mic60 demonstrated that the twisted arrangement of Mic60 can occur within nonconstricted tubules, disproving the hypothesis that the twisted arrangements of Mic60 are limited to mitochondrial constriction sites (Fig. 2E). At the same time, these recordings demonstrate that the Mic60 band patterns are not a simple projection artifact, as such band patterns do not appear in the Tom22 channel. Tom 22 is part of the translocase of the outer membrane (TOM) complex, which has been shown to interact with MICOS in yeast mitochondria $(14,16)$. However, discernible twisted band-like distribution patterns were found only for Mic60 clusters.

To further investigate the distribution of Mic60 in human cells, we immunolabeled human A-498 kidney carcinoma cells for Mic60 or Tom 20 and recorded them with 3D STED nanoscopy (Fig. 2F, SI Appendix, Figs. S2F and S3, and Movie S2). After straightening the mitochondrial tubules in silico, the fluorescence signals of $1.5-\mu \mathrm{m}$-long mitochondrial segments were summed along the longitudinal mitochondrial axis. We analyzed 552 mitochondrial segments and found that in the majority of all segments, the Tom 20 clusters were homogenously distributed without angular preference (Fig. $2 F$ and SI Appendix, Fig. S3 $C-$ $E)$. The added fluorescence signals of segments decorated for Mic60 were clearly different (Fig. $2 F$ and SI Appendix, Fig. S3) In approximately $17 \%$ of all segments, two clear opposing signals were visible (Fig. $2 F$ and SI Appendix, Fig. S $3 F$ ), corresponding to two parallel untwisted bands spanning the entire $1.5-\mu \mathrm{m}$ segments. The remaining fluorescence signal distributions were fully in line with the view of Mic60 arranged in two twisted distribution bands, as the twisting is expected to smear out the summed fluorescence signal into rings with gaps, which was observed in $37 \%$ of all summed segments. This finding of twisted Mic60 bands is further supported by an animated view of consecutive virtual sections rectangular to the longitudinal axis of the mitochondria suggesting that the double-helical patterns originate from the twisting of the two distribution bands (Movie S2). Taken together, these data demonstrate that in different human cell types, Mic60 forms clusters that are enriched in opposing distribution bands, which can adopt a helical arrangement.

The Opposing Distribution Bands of Mic60 Are Largely Independent of the Cristae Morphology. We observed an enrichment of Mic60 in opposing distribution bands in yeast cells (Fig. $1 A$ and $B$ and $S I$ Appendix, Fig. S1 $A$ ), primary HDFas (Fig. $2 A$ and $B$ and $S I$ Appendix, Fig. S2A), and human A-498 kidney carcinoma cells (Fig. $2 F$, and SI Appendix, Fig. S2F, and Movie S2). Under the growth conditions used here (i.e., logarithmically growing cells with galactose as carbon source), the cristae of the yeast cells generally did not fully span the interior of the mitochondria (Fig. $1 C$ and $D$ and SI Appendix, Fig. S1 $E-G$ ), while the fibroblasts featured heterogeneous, tubular, or sheet-like cristae (Fig. $2 D$ and SI Appendix, Fig. S2D). Thus, because the cristae do not necessarily span the matrix space, it appears likely that the opposing arrangement of Mic60 is not determined primarily by the cristae structure.

To further investigate the influence of cristae morphology on Mic60 distribution, we determined the distribution of Mic60 in mitochondria of $\Delta$ mic10 yeast cells, which exhibit aberrant cristae. In $\Delta$ mic10 mitochondria, the cristae membranes are largely detached from the inner boundary membrane, as the number of crista junctions is reduced $(12,15,24,25)$. Thus, if the cristae determine the localization of the Mic 60 clusters, the distribution of these clusters would be predicted to be significantly changed in $\Delta$ mic 10 yeast cells.

Confocal recordings revealed bulbous mitochondria in $\sim 27 \%$ of the $\Delta$ mic10 cells (compared with $\sim 4 \%$ in wild-type cells), but the majority of the cells still displayed tubular mitochondria as well (Fig. $3 A$ and SI Appendix, Fig. S4A), which we used for further analysis. When recorded with STED nanoscopy, Mic60 clusters arranged in opposing distribution bands were visible in a large fraction of the mitochondrial tubules (Fig. 3B). The fraction of cells exhibiting at least one occurrence of twisted Mic60 distribution bands in a 2D STED image was slightly reduced to $30 \%$ in $\Delta m i c 10$ cells compared with $48 \%$ in wild-type cells; in addition, we observed longer parallel Mic60 arrangements without twisting in the $\Delta$ mic10 cells (Fig. $3 B$ and $C$ ). Taken together, these data suggest that the arrangement of the Mic60 clusters at opposing sides of the mitochondria is largely independent of the cristae morphology.

Mic60-Interacting Proteins Also Localize in Distinct Clusters. The MICOS complex is composed of two subcomplexes: the Mic60 subcomplex, which encompasses Mic60 and Mic19, and the Mic10 subcomplex, which includes Mic10, Mic12, Mic26, and Mic27 in yeast $(18,26)$. To investigate whether the Mic10 subcomplexes also exhibit an ordered arrangement within mitochondria, we investigated the nanoscale distributions of Mic10 as well as of GFP-tagged Mic12 and Mic27 in yeast. As seen for Mic60, we found that these three proteins also form distinct clusters that are frequently arranged in patterns similar to those 
A

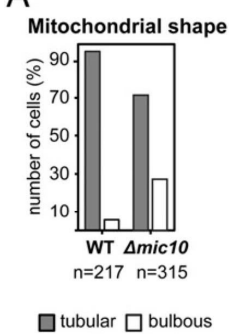

C

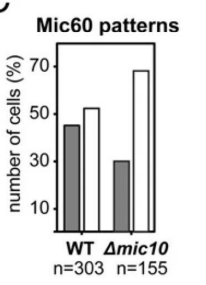

$\square$ twisted $\square$ other

B

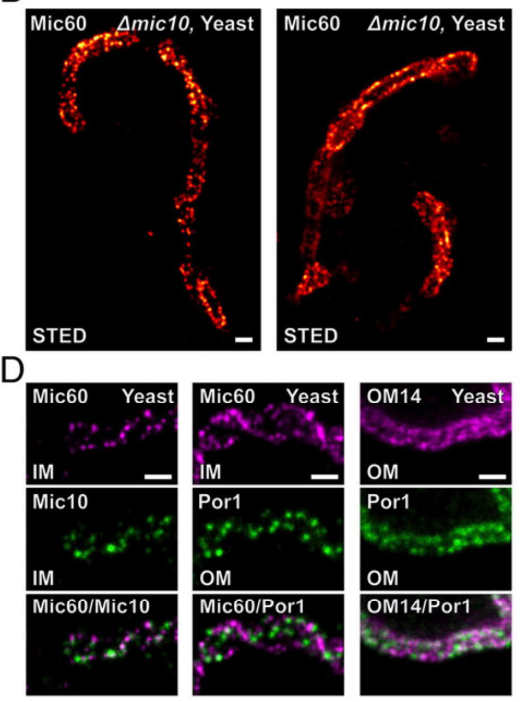

Fig. 3. Nanoscale distribution of yeast mitochondrial inner and outer membrane proteins. (A-C) Mitochondrial shape and Mic60-GFP distribution patterns in WT and $\Delta$ mic10 cells labeled with a GFP-specific antiserum. (A) Quantification of cells with tubular and bulbous mitochondria. (B) STED images of entire $\Delta$ mic10 yeast cells. (C) Quantification of twisted Mic60 distribution patterns in WT and $\Delta m i c 10$ cells. (D) Dual-color STED nanoscopy of mitochondrial membrane proteins, as indicated. Mic10 and Por 1 were labeled with specific antisera. Mic60 and OM14 were expressed from the respective native genomic loci as GFP fusion proteins and decorated with a GFP-specific antiserum. (Scale bars: $400 \mathrm{~nm}$ in $B$ and $D$ ).

seen for Mic60 (Fig. 3D and SI Appendix, Fig. S4B). However, these patterns seemed to be less distinct than those that were recorded when the yeast cells were labeled for Mic60.

Because MICOS has been shown to engage in numerous physical interactions with proteins of the outer membrane in yeast $(12-14,16,20)$, we next analyzed the submitochondrial distributions of several other of these interacting proteins, including OM14, a mitochondrial outer membrane receptor for cytosolic ribosomes; Tom 20 and Tom40, subunits of the translocase of the outer membrane; and Por1, a voltage-dependent anion channel (mitochondrial porin). Intriguingly, we found that all these integral outer membrane proteins were enriched in clusters that appeared to be inhomogeneously distributed, suggesting an ordered arrangement of these proteins as well. However, the heterogeneity of the observed distribution patterns and the uncertainty of the degree of labeling due to the use of polyclonal antibodies with different binding affinities preclude a statistically robust quantitative analysis of the level of molecular interactions. Qualitatively, the distribution patterns of these proteins did not clearly match the helical pattern observed for Mic10 and Mic60 (Fig. $3 D$ and $S I$ Appendix, Fig. S4C). We conclude that the frequent qualitative observations of patterned mitochondrial membrane protein distributions, most notably in the case of Mic60, suggest a large-scale distributional organization of numerous proteins in the mitochondrial inner and outer membranes.

\section{Discussion}

This study shows that MICOS clusters in yeast and in different human cell lines are often distributed on opposing sides of a mitochondrion within distribution bands (Fig. 4). We find that these distribution bands can be twisted, resulting in a helical arrangement of the MICOS clusters (Figs. $1 A$ and $B$ and $2 A$ ). Whereas we primarily observed such helical arrangements in the curved and highly dynamic networks of yeast mitochondria, extended untwisted arrangements are also visible in mammalian cells (Fig. $2 A-C$ ). The latter has been reported previously (23), and here we demonstrate that an untwisted arrangement of the distribution bands can be contiguous with a helical arrangement.

In yeast mitochondria, our FIB-SEM data reveal short lamellar cristae that exhibit a twisted spatial organization. It was recently shown that the cristae of Drosophila indirect flight muscle mitochondria can also adopt a helical arrangement (27). Thus, such helical crista arrangements might be a common architectural feature across several cell types. Nevertheless, the occurrence of such twisted arrangements of cristae and crista junctions seems to be largely independent of the crista shape, as yeast mitochondria have relatively small and short lamellar cristae, while HDFa mitochondria have longer, tubular, or sheetlike cristae, and the helical cristae of Drosophila mitochondria are large lamellar structures. The wide spectrum of crista shapes associated with helical orientations argues against the idea of membrane physics being exclusively responsible for the intricate orientation of the cristae and the distribution of crista junctions.

Mic60 is an ancient protein $(28,29)$ that is enriched at crista junctions and has been demonstrated to bend membranes in vitro $(11,12,23,30,31)$. The rather constant spacing of the crista junctions and localization of the Mic60 clusters in distribution bands that run along the length of the mitochondrial tubules argue for a connection of these clusters along the tubules. Indeed, several findings suggest that the Mic60 clusters are coupled not only along, but also across the mitochondrial tubules, independent of the cristae. First, in yeast cells, the short lamellar cristae do not span the mitochondrial tubules. However, an arrangement of protein clusters in opposing distributions bands is difficult to explain without proposing some kind of physical connection between the clusters across the mitochondrial tubules. Furthermore, the opposing distribution of Mic60 is maintained in $\Delta$ mic10 yeast cells that have aberrant cristae, also arguing for a physical connection of the Mic 60 clusters across the mitochondrial tubules by a structure

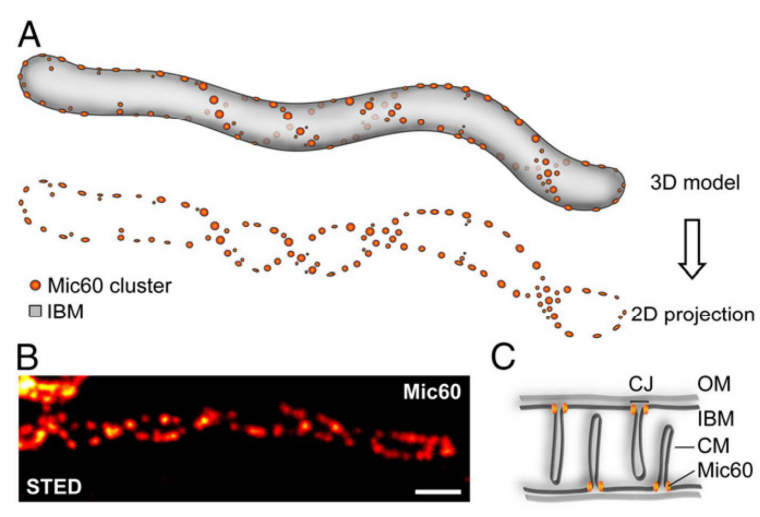

Fig. 4. Mic60 clusters are distributed in two opposing distribution bands of varying widths. (A) Cartoon showing the postulated distribution of Mic60 (Mic60 clusters are depicted as orange spheres) on a mitochondrial tubule. The inner boundary membrane is in gray. Note that the clusters are in opposing distribution bands of varying widths. The twisting of these bands varies, so that both helical and parallel arrangements are observed. Shown is a 3D cartoon (Top) and the projection of the Mic60 clusters into one plane. The latter corresponds to the situation in 2D STED nanoscopy, which, because of its diffraction-limited $z$ resolution, projects the signals from an entire mitochondrion into one plane. (B) For comparison with the cartoon, a 2D STED recording of a moderately twisted Mic60 pattern (same image as in Fig. $2 B$ ) is shown. (C) Sketch of the mitochondrial membrane architecture. Mic60 is preferentially localized at the crista junctions (Cls). CM, cristae membrane; IBM, inner boundary membrane; OM, outer membrane. (Scale bar: $500 \mathrm{~nm}$.) 
within the mitochondrial membranes. Moreover, previous studies have shown that in yeast mitochondria devoid of crista membranes, the punctate labeling pattern of MICOS is not significantly changed $(13,18)$. Finally, our STED data suggest that in mammalian cells, the Mic60 clusters in one distribution band are spatially correlated with the clusters in the opposing band. Taken together, these findings suggest that the MICOS clusters are physically coupled along and across the mitochondrial tubules, presumably by proteins in the mitochondrial inner membrane.

We did not observe a continuous filamentous structure for any of the investigated MICOS subunits in yeast or mammalian cells. Thus, our data give rise to the intriguing idea that Mic60, and some of its interacting proteins, together form a large heterogeneous membrane-embedded structure that scaffolds the organelle.

In line with the idea of MICOS being a part of a scaffolding structure, deletions of the core subunits of the MICOS complex have drastic effects on the architecture of the mitochondrial inner membrane (11-13), as well as on the overall mitochondrial morphology $(13,32,33)$. Mitochondria frequently undergo fusion and fission, thereby changing the network morphology $(1$, 3 ). Thus, any scaffolding structure would be expected to be flexible and heterogeneous. This fits with our finding that even the Mic60 localizations were highly variable, although they provided the clearest indication of the existence of a patterned distribution; therefore, the proposed structure (13) is expected to be flexible and heterogeneous. We predict that it is much less rigid than, for example, the periodic lattice of actin rings evenly spaced by spectrin tetramers found in the plasma membrane of neuronal axons and dendrites $(34,35)$.

1. Bereiter-Hahn J, Vöth M (1994) Dynamics of mitochondria in living cells: Shape changes, dislocations, fusion, and fission of mitochondria. Microsc Res Tech 27:198-219.

2. Sesaki H, Jensen RE (1999) Division versus fusion: Dnm1p and Fzo1p antagonistically regulate mitochondrial shape. J Cell Biol 147:699-706.

3. Shaw JM, Nunnari J (2002) Mitochondrial dynamics and division in budding yeast. Trends Cell Biol 12:178-184.

4. Wasilewski M, Chojnacka K, Chacinska A (2017) Protein trafficking at the crossroads to mitochondria. Biochim Biophys Acta Mol Cell Res 1864:125-137.

5. Merkwirth C, Langer T (2008) Mitofusin 2 builds a bridge between ER and mitochondria. Cell 135:1165-1167.

6. Eisenberg-Bord M, Shai N, Schuldiner M, Bohnert M (2016) A tether is a tether is a 6. Eisenberg-Bord M, Shai N, Schuldiner M, Bohnert M (2016) A tether
tether: Tethering at membrane contact sites. Dev Cell 39:395-409.

tether: Tethering at membrane contact sites. Dev Cell 39:395-409.
7. Martijn J, Vosseberg J, Guy L, Offre P, Ettema TJG (2018) Deep mitochondrial origin 7. Martijn J, Vosseberg J, Guy L, Offre P, Ettema TJG (2018) Deep m
outside the sampled alphaproteobacteria. Nature 557:101-105.

outside the sampled alphaproteobacteria. Nature 557:101-105.
8. Pilhofer M, Jensen GJ (2013) The bacterial cytoskeleton: More than twisted filaments. 8. Pilhofer M, Jensen GJ (2013) The
Curr Opin Cell Biol 25:125-133.

9. Wagstaff J, Löwe J (2018) Prokaryotic cytoskeletons: Protein filaments organizing small cells. Nat Rev Microbiol 16:187-201.

10. Pfanner N, et al. (2014) Uniform nomenclature for the mitochondrial contact site and cristae organizing system. J Cell Biol 204:1083-1086.

11. Rabl R, et al. (2009) Formation of cristae and crista junctions in mitochondria depends on antagonism between Fcj1 and Su e/g. J Cell Biol 185:1047-1063.

12. Harner M, et al. (2011) The mitochondrial contact site complex, a determinant of
on a 2. Harner M, et al. (2011) The mitochondrial contact
mitochondrial architecture. EMBO J 30:4356-4370.

13. Hoppins $\mathrm{S}$, et al. (2011) A mitochondrial-focused genetic interaction map reveals a 3. Hoppins $\mathrm{S}$, et al. (2011) A mitochondrial-focused genetic interaction map reveals a
scaffold-like complex required for inner membrane organization in mitochondria. scaffold-like complex requi
$\mathrm{J}$ Cell Biol 195:323-340.

J Cell Biol 195:323-340.
14. von der Malsburg K, et al. (2011) Dual role of mitofilin in mitochondrial membrane organization and protein biogenesis. Dev Cell 21:694-707.

15. Alkhaja AK, et al. (2012) MINOS1 is a conserved component of mitofilin complexes and required for mitochondrial function and cristae organization. Mol Biol Cell 23:247-257.

16. Bohnert $M$, et al. (2012) Role of mitochondrial inner membrane organizing system in protein biogenesis of the mitochondrial outer membrane. Mol Biol Cell 23:3948-3956.

17. Ott C, et al. (2012) Sam50 functions in mitochondrial intermembrane space bridging and biogenesis of respiratory complexes. Mol Cell Biol 32:1173-1188.

18. Friedman JR, Mourier A, Yamada J, McCaffery JM, Nunnari J (2015) MICOS coordinates with respiratory complexes and lipids to establish mitochondrial inner membrane architecture. elife 4:e07739.

19. Harner ME, et al. (2016) An evidence-based hypothesis on the existence of two pathways of mitochondrial crista formation. eLife 5:e18853.
Remarkably, yeast mitochondria devoid of both crista membranes and the MICOS complex have a tubular shape (18), suggesting that additional factors independent of MICOS contribute to the structural rigidity of the organelle. Indeed, MICOS interacts with a multitude of proteins in both mitochondrial membranes, which might be part of this structure (12, $13,15-20)$. Thus, we suggest the existence of a structurally variable multiprotein structure in the mitochondrial inner membrane and potentially the outer membrane that scaffolds the organelle. We propose that this structure is the elusive mitoskeleton.

\section{Materials and Methods}

A detailed description of the methodology of this study, including the generation of yeast strains, immunofluorescent labeling of yeast cells, culturing of mammalian cell lines, 2D and 3D STED nanoscopy, statistical analysis, FIB-SEM of yeast cells, and TEM of HDFa cells, is provided in SI Appendix, Materials and Methods.

ACKNOWLEDGMENTS. We thank Anna Kremer and Saskia Lippens (VIB Biolmaging Core) for acquisition of the FIB-SEM data; Peter llgen for help with $3 \mathrm{D}$ animations: with 3D animations; Rita Schmitz-Salue and Gudrun Heim for excellent
technical assistance; Peter Rehling for antisera specific to Tom40 and Mic10; technical assistance; Peter Rehling for antisera specific to Tom40 and Mic10; Valentina Carannante for the A-498 cells and cell culture support; Hans Blom
for STED support; Benedikt Westermann for the plasmid pVT100U-mt GFP; and Jaydev Jethwa for a careful reading of the manuscript. Infrastructure development support was provided by the Swedish Foundation for Strategic Research (RIF14-0091, to D.C.J.). This work was supported by the German Research Foundation-funded SFB1190 (project P01, to S.J.).

20. Rampelt $H$, Zerbes RM, van der Laan M, Pfanner N (2017) Role of the mitochondrial contact site and cristae organizing system in membrane architecture and dynamics. Biochim Biophys Acta Mol Cell Res 1864:737-746.

21. Huang $B$, Babcock $H$, Zhuang $X$ (2010) Breaking the diffraction barrier: Superresolution imaging of cells. Cell 143:1047-1058.

22. Sahl SJ, Hell SW, Jakobs S (2017) Fluorescence nanoscopy in cell biology. Nat Rev Mol Cell Biol 18:685-701.

23. Jans DC, et al. (2013) STED super-resolution microscopy reveals an array of MINOS clusters along human mitochondria. Proc Natl Acad Sci USA 110:8936-8941.

24. Barbot M, et al. (2015) Mic10 oligomerizes to bend mitochondrial inner membranes at cristae junctions. Cell Metab 21:756-763.

25. Bohnert M, et al. (2015) Central role of Mic10 in the mitochondrial contact site and cristae organizing system. Cell Metab 21:747-755.

26. Guarani V, et al. (2015) QIL1 is a novel mitochondrial protein required for MICOS

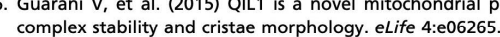

27. Jiang YF, et al. (2017) Electron tomographic analysis reveals ultrastructural features of mitochondrial cristae architecture which reflect energetic state and aging. Sci Rep 7: 45474.

28. Muñoz-Gómez SA, et al. (2015) Ancient homology of the mitochondrial contact site and cristae organizing system points to an endosymbiotic origin of mitochondria cristae. Curr Biol 25:1489-1495.

29. Huynen MA, Mühlmeister M, Gotthardt K, Guerrero-Castillo S, Brandt U (2016) Evolution and structural organization of the mitochondrial contact site (MICOS) complex and the mitochondrial intermembrane space bridging (MIB) complex. Biochim Biophys Acta 1863:91-101.

30. Tarasenko D, et al. (2017) The MICOS component Mic60 displays a conserved membrane-bending activity that is necessary for normal cristae morphology. $J \mathrm{Ce} / \mathrm{I}$ Biol 216:889-899.

31. Hessenberger $M$, et al. (2017) Regulated membrane remodeling by Mic60 controls formation of mitochondrial crista junctions. Nat Commun 8:15258.

32. John GB, et al. (2005) The mitochondrial inner membrane protein mitofilin controls cristae morphology. Mol Biol Cell 16:1543-1554.

33. Xie J, Marusich MF, Souda P, Whitelegge J, Capaldi RA (2007) The mitochondrial inner membrane protein mitofilin exists as a complex with SAM50, metaxins 1 and 2 , coiledcoil-helix coiled-coil-helix domain-containing protein 3 and 6 and DnaJC11. FEBS Lett 581:3545-3549

34. Xu K, Zhong G, Zhuang $X$ (2013) Actin, spectrin, and associated proteins form a periodic cytoskeletal structure in axons. Science 339:452-456.

35. D'Este E, Kamin D, Göttfert F, El-Hady A, Hell SW (2015) STED nanoscopy reveals the ubiquity of subcortical cytoskeleton periodicity in living neurons. Cell Rep 10 1246-1251. 


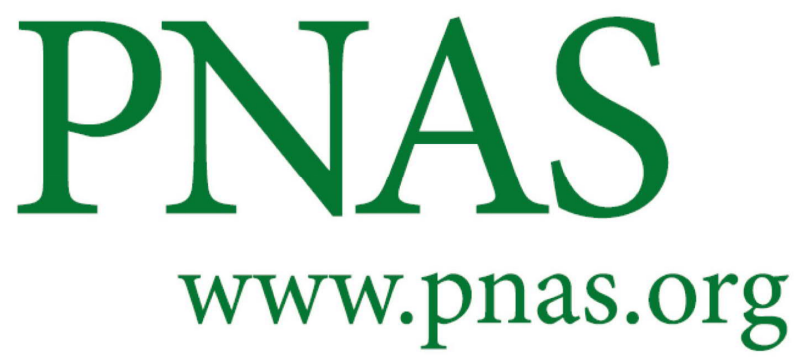

\section{Supplementary Information for}

\section{Mic60 exhibits a coordinated clustered distribution along and across yeast and mammalian mitochondria}

Stefan Stoldt ${ }^{\mathrm{a}, 1}$, Till Stephan ${ }^{\mathrm{a}, 1}$, Daniel C. Jans ${ }^{\mathrm{b}, \mathrm{c}}$, Christian Brüser ${ }^{\mathrm{a}}$, Felix Lange ${ }^{\mathrm{a}}$, Jan Keller-Findeisen ${ }^{\mathrm{a}}$, Dietmar Riedel ${ }^{\mathrm{d}}$, Stefan W. Hell ${ }^{\mathrm{a}}$, and Stefan Jakobs ${ }^{\mathrm{a}, \mathrm{b}, 2}$

${ }^{1}$ S.S. and T.S. contributed equally to this work

${ }^{2}$ To whom correspondence may be adressed. Email: sjakobs@gwdg.de

This PDF file includes:

SI Materials and Methods

Figs. S1 to S4

Tables S1 to S4

Captions for movies S1 and S2

References for SI reference citations

Other supplementary materials for this manuscript include the following:

Movies S1 and S2 


\section{SI Materials and Methods}

\section{Yeast strains, genomic tagging and growth conditions}

For the analysis of sub-mitochondrial protein distributions in the yeast Saccharomyces cerevisiae either GFP fusion strains from the Yeast GFP Clone Collection (BY4741; Cat. No.: 95702, Invitrogen, Carlsbad, CA, USA) or newly tagged strains isogenic to the wild type strain W303 were utilized. Genomic tagging to generate GFP fusions of the proteins of interest was achieved by directed homologous recombination of a PCR product using a HIS3 cassette for positive selection as described previously (36) (Table S1 and S2). A TEV protease specific cleavage site was introduced between the linker and the EGFP sequence of pYM28 (37) to generate the pYM28-spacer-TEV-GFP template plasmid for genomic tagging. All epitope tagged strains were verified by PCR. Only yeast strains expressing full-length GFP fusion proteins and showing normal growth on a non-fermentable carbon source were used for this study. For targeted gene disruption of MIC10 a PCR fragment was generated using genomic DNA isolated from a $\triangle$ mic 10::kanMX4 strain (Table $\mathrm{S} 1$ and S3). The gene disruption was performed as described previously (36). Cultivation of yeast cells was performed according to standard protocols on agar plates or in liquid medium containing $2 \%(\mathrm{w} / \mathrm{v})$ glucose or $2 \%(\mathrm{w} / \mathrm{v})$ galactose, respectively.

\section{Immunofluorescence labelling of yeast cells}

Yeast cells were grown to the early exponential growth phase $\left(\mathrm{OD}_{600 \mathrm{~nm}}=0.4-0.7\right)$ in liquid medium containing $2 \%$ galactose as the sole carbon source. The cells were fixed with $3.7 \%$ formaldehyde in the growth medium at room temperature (RT, $20 \mathrm{~min}$ ), spun down (800 x $\mathrm{g}, 3 \mathrm{~min})$ and washed with phosphate buffered saline with sorbitol (PBS/sorbitol) (137 mM NaCl; 3 mM KCl; $8 \mathrm{mM} \mathrm{Na}_{2} \mathrm{HPO}_{4} ; 1.5 \mathrm{mM} \mathrm{KH}_{2} \mathrm{PO}_{4} ; 10 \%$ (w/v) sorbitol; $\mathrm{pH} 7$ ). Afterwards, cell walls were partially removed by zymolyase treatment $\left(15 \mathrm{~min}, 30^{\circ} \mathrm{C}\right)$. Subsequently, the cells were washed with PBS/sorbitol. Cells were bound to the surface of poly-L-lysine coated cover slips (RT, $30 \mathrm{~min}$ ) and blocked with a blocking solution containing $2 \%(\mathrm{w} / \mathrm{v}$ ) bovine serum albumin (BSA), 0,4\% (w/v) SDS, $0.1 \%$ (v/v) Tween20 in PBS/sorbitol (RT, $60 \mathrm{~min}$ ). Detection of specific epitopes was achieved by incubation of the samples with primary antibodies $\left(4^{\circ} \mathrm{C}\right.$, overnight; Table S4). Following 
two further washing steps with blocking solution, cells were decorated with secondary antibodies custom-labeled with Abberior STAR RED (Abberior, Göttingen, Germany) or Alexa Fluor 594 (Thermo Fisher Scientific, Waltham, MA, USA) (RT, 90 min). After several additional washing steps in blocking solution and PBS/sorbitol, the samples were mounted in Mowiol containing 1,4-Diazabicyclo[2.2.2]octan (DABCO). For all antibodies see Table S4.

\section{Cell culture and immunofluorescence staining of human primary dermal fibroblasts and human kidney carcinoma cells}

Human kidney carcinoma cells (A-498) were cultured in DMEM, high glucose, pyruvate (Thermo Fisher Scientific) supplemented with Penicillin-Streptomycin (Sigma Aldrich, St. Louis, MO, USA) and $10 \%$ (v/v) fetal bovine serum (Thermo Fisher Scientific).

Human primary dermal fibroblasts (HDFa, ATCC, Manassas, VA, USA) were cultured in DMEM containing 4,5 g/L Glucose and GlutaMAX ${ }^{\mathrm{TM}}$ additive (Thermo Fisher Scientific) supplemented with $100 \mathrm{U} / \mathrm{ml}$ penicillin and $100 \mu \mathrm{g} / \mathrm{mL}$ streptomycin (Merck Millipore, Burlington, MA, USA), $1 \mathrm{mM}$ sodium pyruvate (Sigma Aldrich) and $10 \%(\mathrm{v} / \mathrm{v})$ fetal bovine serum (Merck Millipore).

Cells were seeded on coverslips and cultured for 1-3 days $\left(37{ }^{\circ} \mathrm{C}, 5 \% \mathrm{CO}_{2}\right)$. For immunolabeling, cells were fixed with a pre-warmed solution of $4 \%$ formaldehyde in PBS (137 mM NaCl, $2.68 \mathrm{mM} \mathrm{KCl}$ and $10 \mathrm{mM} \mathrm{Na}_{2} \mathrm{HPO}_{4}, \mathrm{pH}$ 7.4) (37 $\left.{ }^{\circ} \mathrm{C}, 5 \mathrm{~min}\right)$.

Cells were extracted with $0.5 \%(\mathrm{v} / \mathrm{v})$ Triton X-100 in PBS and afterwards blocked with $5 \%$ (w/v) BSA in PBS/Glycine (0.1 M) (RT, $10 \mathrm{~min})$. Mic60 was labeled using primary antibodies specific for Mic60/IMMT (Proteintech, Rosemont, IL, USA). HDFas were labeled for Tom22 with specific primary antibodies (Miltenyi Biotec, Bergisch Gladbach, Germany). A-498 cells were labeled for Tom20 using a mixture of different primary antibodies against Tom20 (Santa Cruz Biotechnology, Dallas, TX, USA and Abcam, Cambridge, UK). The primary antibodies were diluted in $5 \%$ BSA (w/v) in PBS/Glycine $(0.1 \mathrm{M})$ and applied to the samples (RT, 1h). After five washing steps with PBS and blocking with $5 \%$ BSA in PBS/Glycine $(0.1 \mathrm{M})$, the primary antibodies were detected with secondary goat anti-rabbit or sheep anti-mouse antibodies (Jackson Immuno Research Laboratories, West Grove, PA, USA) custom-labeled with Abberior STAR RED 
(Abberior) or Alexa Fluor 594 (Thermo Fisher Scientific) or secondary goat antibodies labeled with Alexa Fluor 555 (Abcam) (RT, 1h). The samples were washed five times with PBS and mounted in Mowiol mounting medium containing $0.1 \%$ (w/v) DABCO (Sigma Aldrich). For all antibodies see Table S4.

\section{D STED nanoscopy}

2D STED nanoscopy was performed using a $775 \mathrm{~nm}$ quad scanning STED microscope (Abberior Instruments, Göttingen, Germany) equipped with a UPlanSApo 100x/1,40 Oil objective (Olympus, Tokyo, Japan) and a Katana-08 HP laser (Onefive GmbH, Regensdorf, Switzerland) utilizing a pixel size of $15 \mathrm{~nm}$. The fluorophore Alexa Fluor 594 was excited at $594 \mathrm{~nm}$, Abberior STAR RED was excited at $640 \mathrm{~nm}$ and STED was performed at $775 \mathrm{~nm}$ wavelength. 2D STED data were linearly deconvolved using the Imspector software (Abberior Instruments).

\section{D STED nanoscopy}

3D STED nanoscopy was performed on a Leica TCS SP8 STED 3X (Leica Microsystems, Wetzlar, Germany) equipped with a HC PL APO 100X/1.40 Oil STED WHITE objective. The fluorophore Alexa Fluor 555 was excited at $521 \mathrm{~nm}$ and STED was performed at $660 \mathrm{~nm}$. The 3D STED data were deconvolved using Huygens deconvolution software (Scientific Volume Imaging, Hilversum, Netherlands) and rendered (volume rendition) and animated using Imaris (Bitplane, Zurich, Switzerland).

\section{Quantification of twisted and parallel distribution bands}

To quantify the occurrence of different Mic60 distribution patterns in HDFa cells, STED recordings were manually analyzed. Three categories for the analysis were pre-defined: 'twisted bands', 'parallel bands' and 'not assigned'. On the STED images, all mitochondrial tubules were independently assigned by three test persons to one of the three categories. Contiguous mitochondrial tubules could consist out of sections assigned to different categories. No specifications were given for the length of the mitochondrial sections that were assigned. The total length of mitochondrial tubules assigned to the 
different categories were analyzed by a computer program and the relative amount of the different categories with respect to the length of the entire network were determined.

\section{Averaging of 3D sections of straightened mitochondria imaged with 3D STED microscopy}

Recorded 3D STED data stacks with a pixel size of $22.5 \mathrm{~nm}$ x $22.5 \mathrm{~nm}$ x $40 \mathrm{~nm}$ were deconvolved with a 3D Gaussian peak function of $150 \mathrm{~nm}$ FWHM in all directions applying the Richardson-Lucy algorithm for 20 iterations. Then they were visually inspected and single, straight sections of the mitochondrial network were cut out. Further processing was performed with a custom written script in Matlab (Mathworks, Natick, USA). First, the present section of the mitochondrial network was rotated to be parallel with the $\mathrm{x}$-axis of the coordinate system by visually determining a rotation angle in the $\mathrm{xy}$ plane. Correcting the mitochondrial tube for a shift along the z-axis was also possible, but the chosen rotation angles in the yz plane were typically $<1^{\circ}$. A linear $3 \mathrm{D}$ interpolation on a rotated grid of cubic pixels with a size of $10 \mathrm{~nm}$ resulted in the mitochondrial section being parallel with the $\mathrm{x}$-axis and only slightly bended. The section was further straightened by binning slices 10 times along the $\mathrm{x}$ direction and correlating the binned slices with each other. The maximum of this $2 \mathrm{D}$ correlation gave rise to a shift in the $\mathrm{yz}$ plane that could be applied inversely to stack the mitochondrial slices along the $\mathrm{x}$ direction without any residual systematic bend. A desired length of the straightened 3D section of mitochondria could then be added up.

For Fig. S3, the straightened tubules were automatically segmented into sections of 1.5 $\mu \mathrm{m}$, each overlapping with the next by $750 \mathrm{~nm}$. Subsequently, the fluorescence signal of each section along the longitudinal mitochondrial axis (over $1.5 \mu \mathrm{m}$ ) was added up. All added-up sections were manually assigned to different categories.

\section{Cluster and correlation analysis of 2D sections of straightened mitochondria imaged with 2D STED microscopy}

The recorded 2D STED data had a pixel size of $15 \mathrm{~nm}$ x $15 \mathrm{~nm}$. Data analysis was performed using custom written Matlab scripts. 
First, rectangular regions of interest (ROI) were determined by visually inspecting the 2D images. The ROI rectangle could be rotated and the rotation was chosen so that the interesting section of the mitochondrial network was mostly parallel to one border of the rectangle.

In a next step, the centerline of the section of mitochondria in each ROI was determined. This was performed by smoothing the image with an asymmetrical 2D Gaussian peak function of $500 \mathrm{~nm}$ FWHM longitudinally along the mitochondria and $100 \mathrm{~nm}$ FWHM across. A line detection algorithm, detecting lines of local maxima could reliably detect the centerline of mitochondria on the smoothed data. The centerline itself was also quite smooth.

A perpendicular grid was placed around that centerline by calculating lines vertical to the center line in regular distances $(10 \mathrm{~nm})$ and placing grid points in regular distances $(10 \mathrm{~nm})$ on these vertical lines. A linear 2D interpolation of the original 2D STED data on the grid around the centerlines of the mitochondria in the ROIs resulted in straightened mitochondria sections. These straightened sections were placed in a continuous row by adjusting the vertical position of each section so that their centers of mass in the vertical direction are equal.

This also allowed separating the mitochondria sections unambiguously into two sides. If they are aligned in a row that would be an upper and a lower side. Adding up the upper and the lower side in the direction across the mitochondria resulted in two intensity profiles for the two sides of all obtained sections combined. The Pearson correlation coefficient between those two profiles could be computed. An error for the correlation coefficient could be computed by bootstrapping. As a control, the correlation between the two sides was computed with one side shifted along the mitochondria for a small distance $(\sim 500 \mathrm{~nm})$ and resulted in no apparent correlation.

The combined, straightened sections of mitochondria predominantly showed a chain of single clusters on each side of the mitochondria. In order to estimate the average distance along the mitochondria between two neighbored clusters for each side of the mitochondria, the following procedure was applied. First, the combined sections were deconvolved with a 2D Gaussian peak of $50 \mathrm{~nm}$ FWHM using 10 iterations of the Richardson-Lucy algorithm. Then the clusters were localized by identifying local maxima. The two sides of 
the mitochondria were separated and only the position of the local maxima along the mitochondria was noted. The distances between consecutive positions gave the distances between neighbored clusters, where only consecutive positions within the same original section were regarded and the results of both sides of the mitochondria were aggregated. Please note that because of the finite resolution the estimated distance between neighbored clusters is limited to a distance of approximately $>50 \mathrm{~nm}$.

\section{Potassium permanganate fixation of yeast cells, Focused Ion Beam milling combined with Scanning Electron Microscopy (FIB-SEM) and subsequent 3D reconstruction of the cristae morphology}

$S$. cerevisiae cells were grown to the early exponential growth phase $\left(\mathrm{OD}_{600 \mathrm{~nm}}=0.4-0.7\right)$ in liquid medium containing galactose as the sole carbon source and harvested by centrifugation. Cells were fixed utilizing $2 \%$ glutaraldehyde in $0.1 \mathrm{M}$ sodium cacodylate buffer at pH 7.4 (RT, $30 \mathrm{~min}$ ). Subsequently, the cells were pelleted and postfixed with $1.5 \%$ potassium permanganate (RT, $2 \mathrm{~h}$ ). After that the cells were washed with distilled water (3x) and resuspended in $2 \%$ agarose. The resulting agarose block was carefully partitioned, stained with $1 \%$ uranyl acetate, dehydrated consecutively by increasing ethanol concentrations and embedded in Agar 100 resin (Agar Scientific, Essex, UK).

In preparation for FIB-SEM imaging, the embedded cells were mounted on aluminium SEM stubs (diameter $12 \mathrm{~mm}$ ) and coated with $\sim 8 \mathrm{~nm}$ of Platinum (Quorum Q150T ES, Lewes, UK). The Zeiss Crossbeam 540 system in combination with the Atlas5 software (Carl Zeiss, Oberkochen, Germany) was utilized for FIB-SEM imaging. Consecutive sections of $5 \mathrm{~nm}$ were removed by FIB milling (via propelling Gallium ions at the sample surface; probe current: $700 \mathrm{pA}$ ). Image acquisition was performed at $1.5 \mathrm{kV}$ and $600 \mathrm{pA}$ using an ESB (back-scattered electron) detector, with the ESB grid set at $-1200 \mathrm{~V}$. The resulting data set was segmented semiautomatically and reconstructed using the software package IMOD (http://bio3d.colorado.edu/imod/) and Amira for Life Sciences (Thermo Fisher Scientific) software. 


\section{Transmission electron microscopy of human fibroblasts}

For transmission electron microscopy, HDFa cells were grown on Aclar discs (Plano, Wetzlar, Germany) to a confluence of $\sim 50 \%$ and fixed with prewarmed $2.5 \%$ glutaraldehyde in $0.1 \mathrm{M}$ cacodylate buffer ( $\mathrm{pH}$ 7.4) (RT, 1h). Fixation was completed overnight $\left(4{ }^{\circ} \mathrm{C}\right)$. After washing three times with $0.1 \mathrm{M}$ cacodylate buffer the cells were stained in $1 \%(\mathrm{w} / \mathrm{v})$ osmium tetroxide in $0.1 \mathrm{M}$ cacodylate buffer (RT, $1 \mathrm{~h})$.

The cells were washed with distilled water ( 3 times each for $5 \mathrm{~min}$ ) and stained en-bloc in aqueous $1 \%(\mathrm{w} / \mathrm{v})$ uranyl acetate in the dark (RT, $30 \mathrm{~min})$. After dehydration in an ethanol series of 30, 50, 70 and $100 \%$ (three times each for $5 \mathrm{~min}$ ) with a final dehydration step in propylene oxide (5 min) the cells were embedded in Agar 100 epoxy resin. Sections of 50 $\mathrm{nm}$ thickness were recorded on a Philips CM120 transmission electron microscope at $15000 \times$ magnification with a TVIPS $2 \mathrm{k} \times 2 \mathrm{k}$ slow-scan CCD camera. 
Figure S1
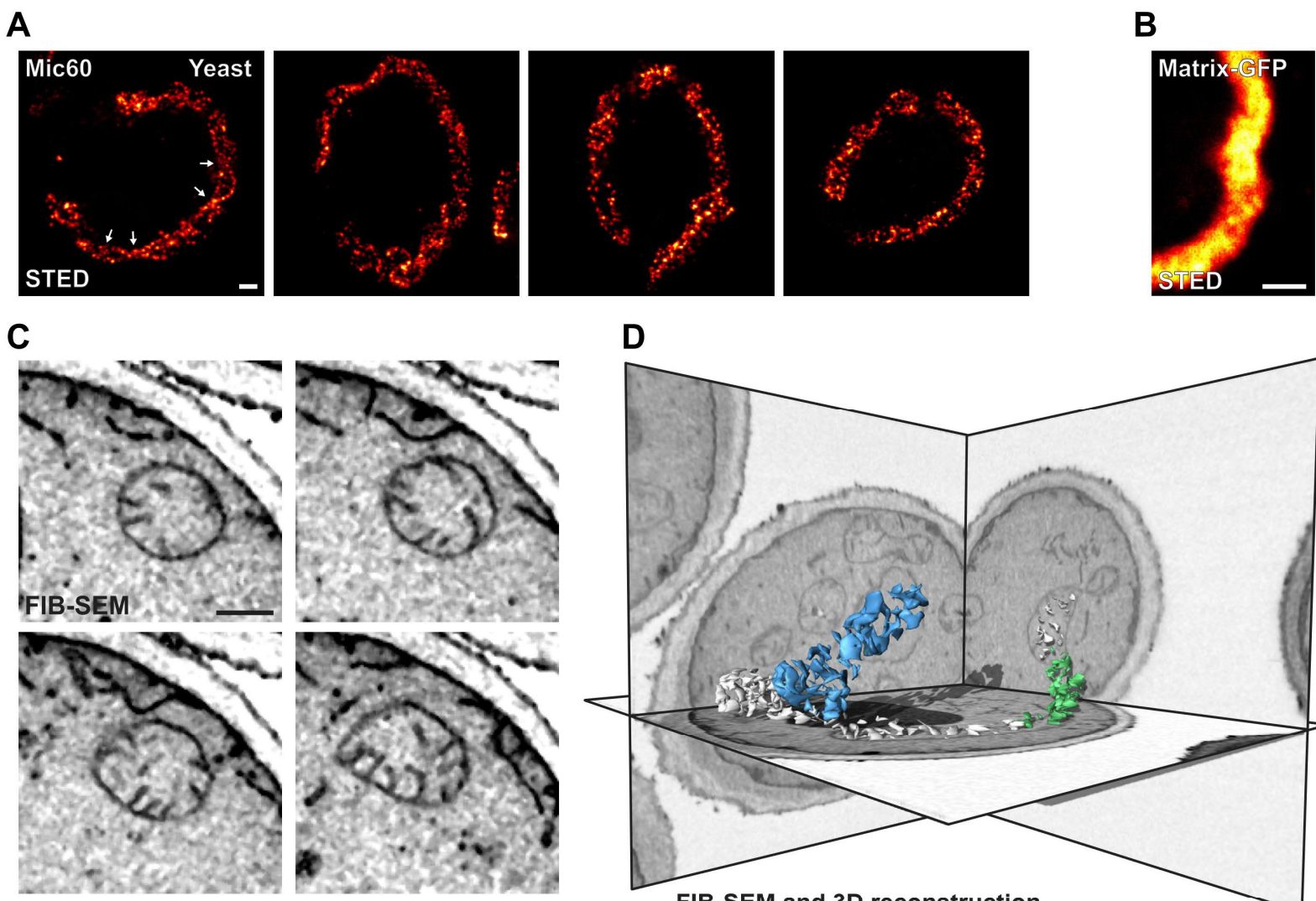

E
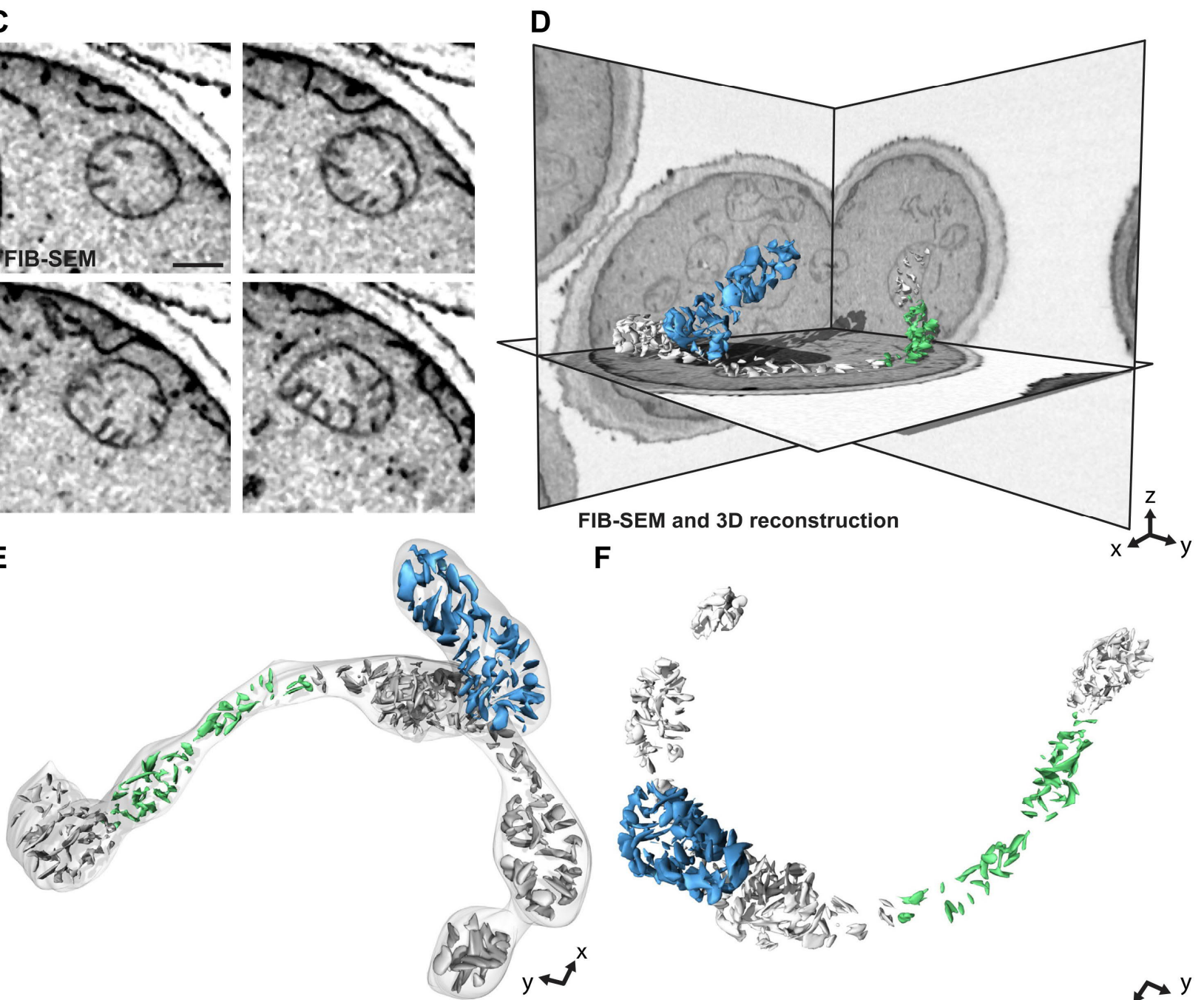

ans

G
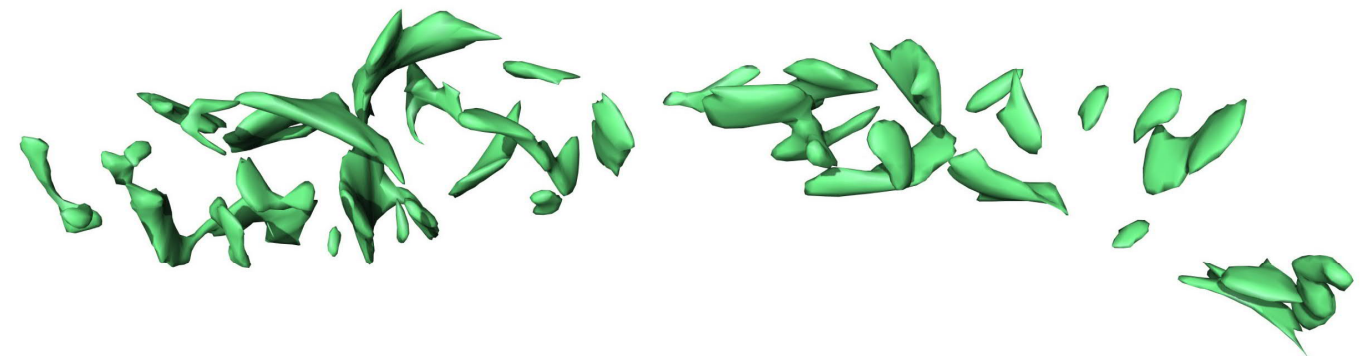
Fig. S1. Mic60 and the cristae are often arranged in a helical pattern in budding yeast mitochondria.

A. STED nanoscopy of entire yeast cells expressing Mic60-GFP fusion proteins. Cells were labeled with a GFP-specific antiserum.

B. STED nanoscopy of matrix targeted GFP in the mitochondria of $S$. cerevisiae. Cells were labeled with a GFP-specific antiserum.

C. Slices of a mitochondrion from wild type budding yeast recorded by FIB-SEM. S. cerevisiae cells grown with galactose as the sole carbon source to the early logarithmical phase at $30^{\circ} \mathrm{C}$ were chemically fixed and analyzed by FIB-SEM. Shown are four slices of the mitochondrion which is depicted in Figs. 1 C,D.

D. Ortho slice view and part of the reconstructed cristae of the cell shown in C. Images show a FIB-SEM stack with one yeast cell at its center and highlight the cellular position of the mitochondrion depicted in Figs. 1 C,D.

E-G. Different views on parts of the reconstructed cristae of the cell shown in C. The part used for the rendition shown in Figs. 1 C,D is depicted in blue.

With the exception of contrast stretching no further image processing was applied to the STED images. Scale bars: $400 \mathrm{~nm}$ (A, B), $150 \mathrm{~nm}(\mathrm{C}, \mathrm{G})$. 
Figure S2

A

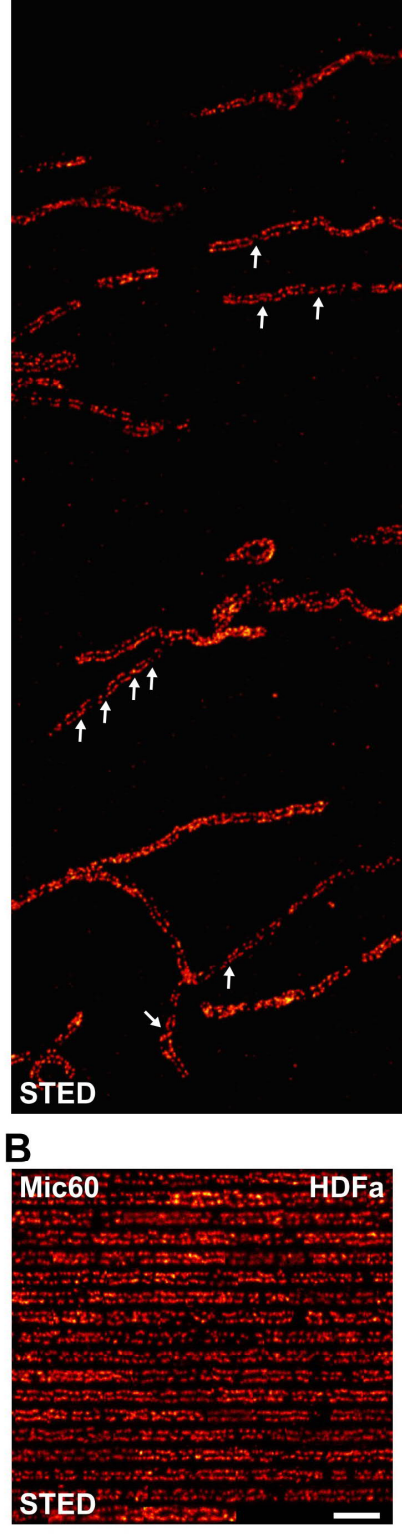

D

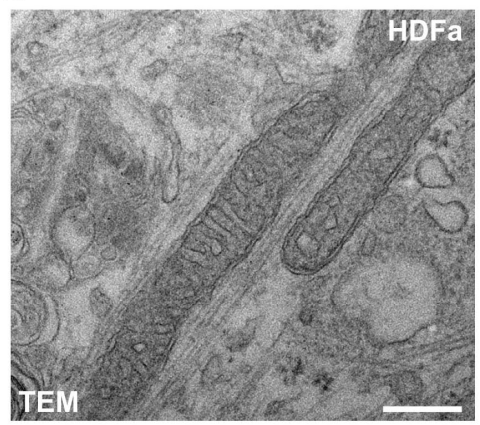

C

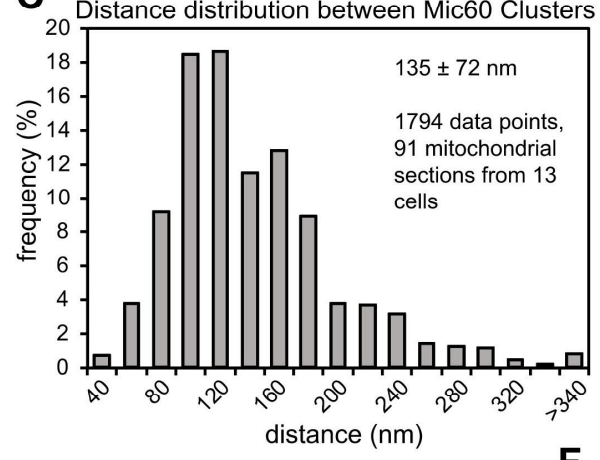

$F$

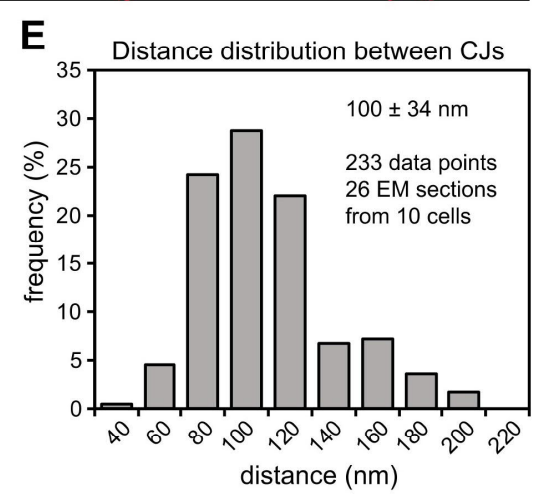

E

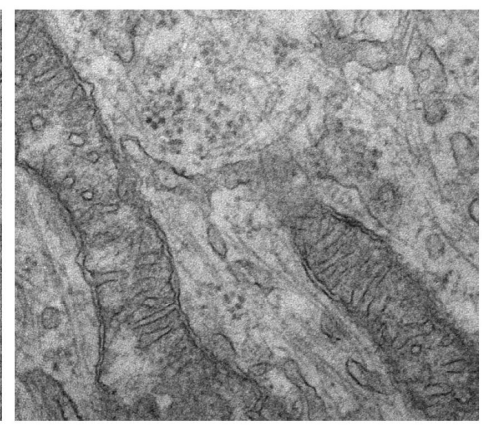

Mic60

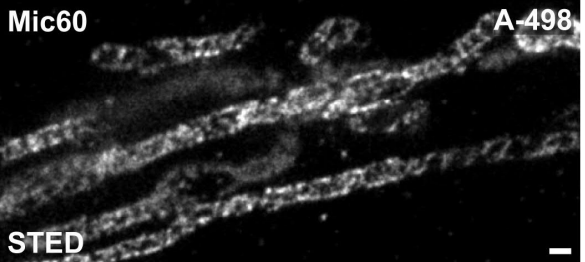


Fig. S2. Mic60 exhibits a scaffold-like distribution in the mitochondria of mammalian cells

A. STED nanoscopy of Mic60 in HDFas. Cells were labeled with a Mic60 antiserum and recorded by STED nanoscopy. Mitochondrial segments showing twisted Mic60 patterns are marked by arrows.

B-C. HDFa cells were labeled with a Mic60 antiserum and recorded by STED nanoscopy. The labeled mitochondria were automatically identified and straightened in silico (B). Subsequently, the distance distribution between Mic60 clusters along one side of the mitochondrial tubules were determined (C).

D-E. Measurement of the distance distribution of cristae junctions along one side of the mitochondrial tubules in HDFa cells (E) based TEM images (D).

F. Spatial distribution of Mic60 in mitochondria from A-498 cells. Human A-498 cells were labeled with a specific antiserum against Mic60 and recorded by STED nanoscopy. Shown are raw data.

With the exception of contrast stretching no further image processing was applied. Scale bars: $1 \mu \mathrm{m}(\mathrm{A}, \mathrm{B}) ; 200 \mathrm{~nm}(\mathrm{D}, \mathrm{F})$. 


\section{Figure S3}
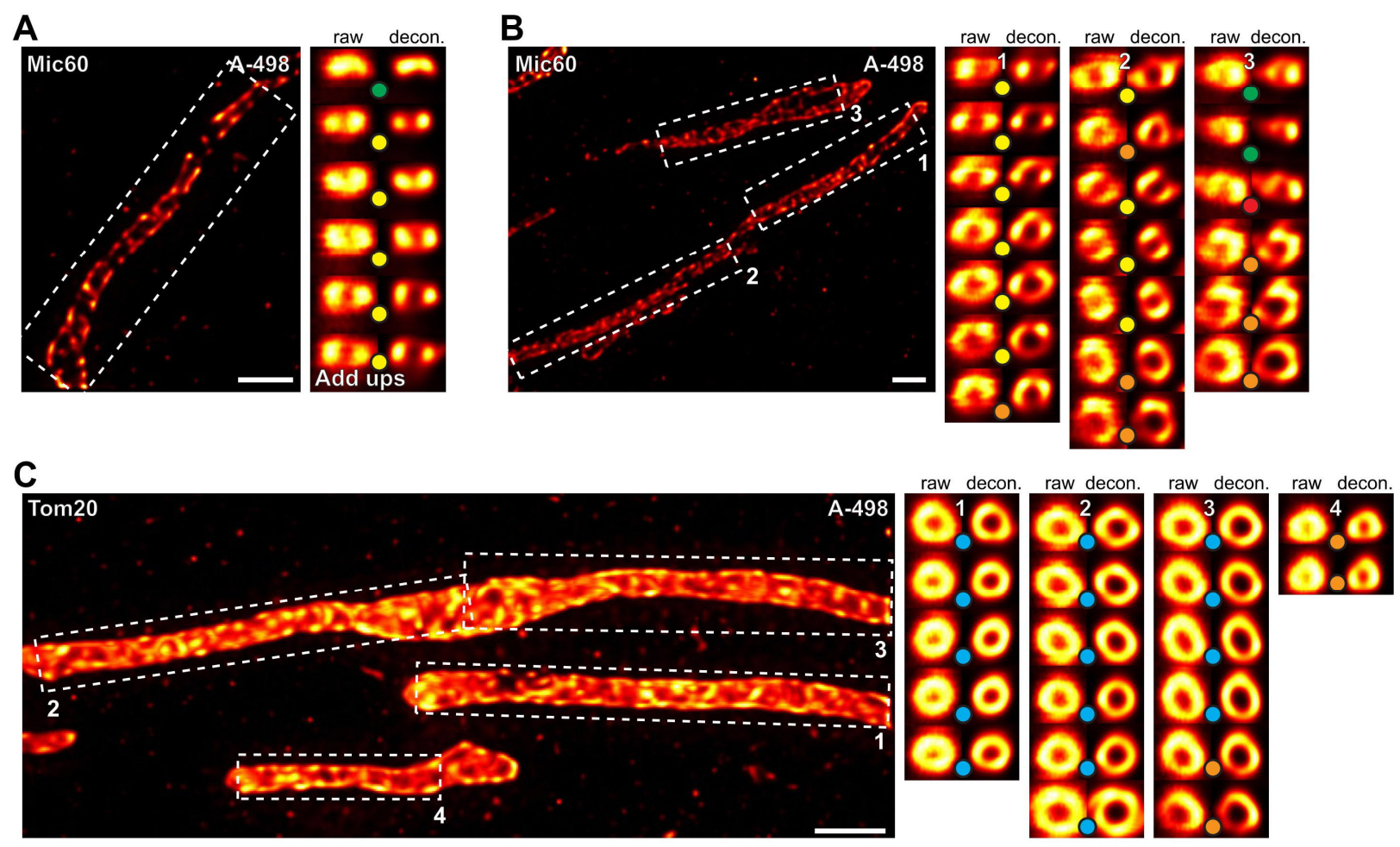

D

Protein distribution in add ups

Protein distribution shapes

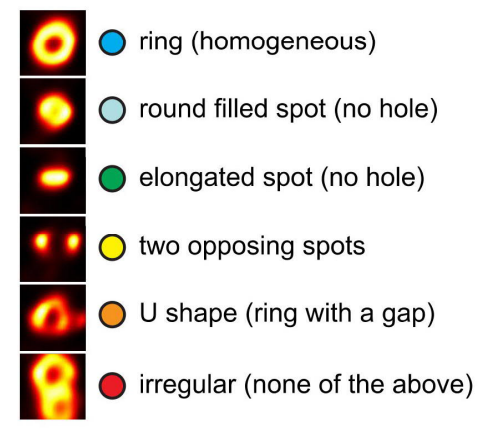

E

\begin{tabular}{|c|}
\hline Tom20 \\
{$[\%]$} \\
55.7 \\
10.4 \\
8.0 \\
2.0 \\
19.9 \\
4.0 \\
$n=201$ \\
\hline
\end{tabular}

F

\begin{tabular}{|c|}
\hline Mic60 \\
{$[\%]$} \\
6.6 \\
15.1 \\
18.8 \\
17.1 \\
37.0 \\
5.7 \\
$n=351$ \\
\hline
\end{tabular}


Fig. S3. Mic60 is localized in opposing distribution bands in human mitochondria A-B. 3D STED nanoscopy of Mic60 in A-498 cells. Left: One optical section of a 3D STED image of mitochondria of A-498 cells decorated for Mic60. Right: Longitudinally added Mic60 fluorescence signals from the mitochondrial segments as indicated in the corresponding STED image. Each segment covers $1.5 \mu \mathrm{m}$. To add the fluorescence intensities, the straightened tubules were automatically segmented into parts of $1.5 \mu \mathrm{m}$, each overlapping with the next by $750 \mathrm{~nm}$. Subsequently, the fluorescence signal of each segment along the longitudinal mitochondrial axis (over $1.5 \mu \mathrm{m}$ ) was added up. Segments were manually assigned to different categories. The assigned categories are indicated by the color-coded spheres.

C. 3D STED nanoscopy of Tom 20 in A-498 cells. The images were processed and analyzed as detailed for A-B.

D. Explanation of the color coding used for assignment. Shown are representative images for each category.

E-F. Statistical evaluation of segments with added up fluorescence signals. Analyzed were 201 Tom20, and 351 Mic60 segments. Scale bars: $1 \mu \mathrm{m}$ (A-C). 
Figure S4
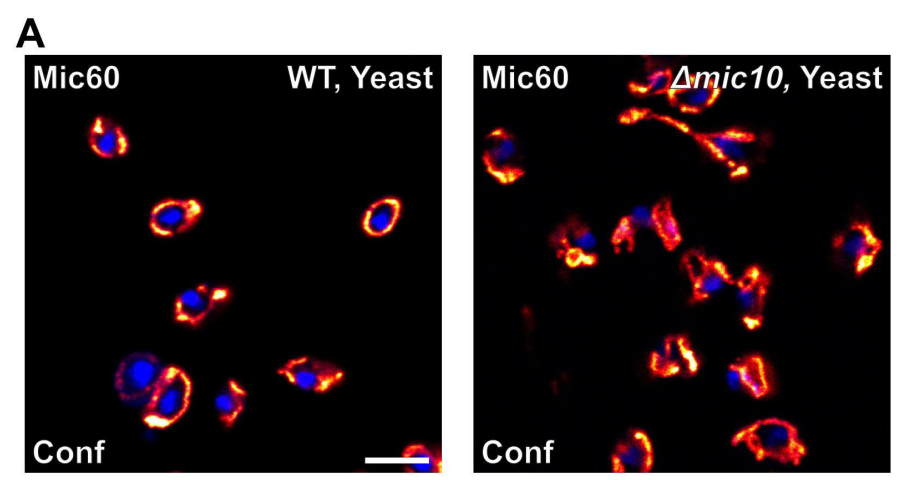

B
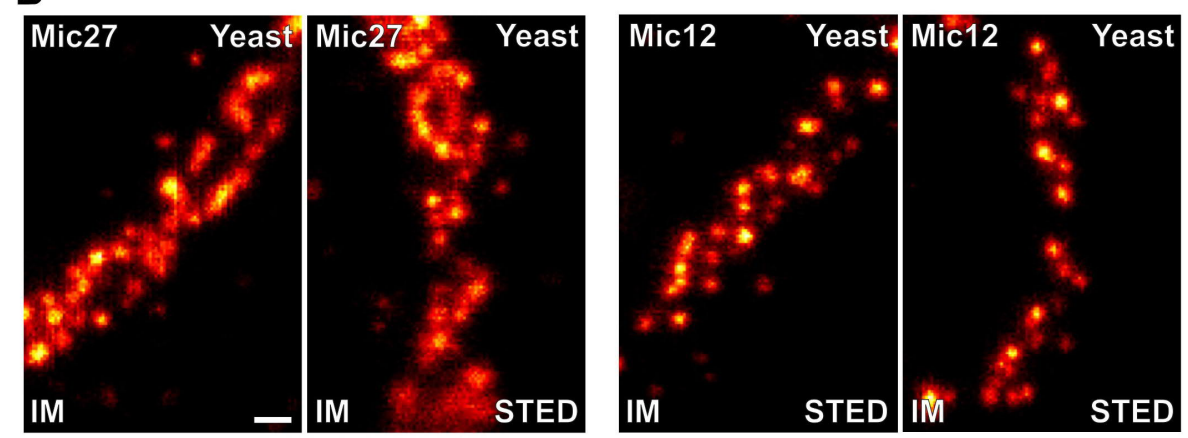

C
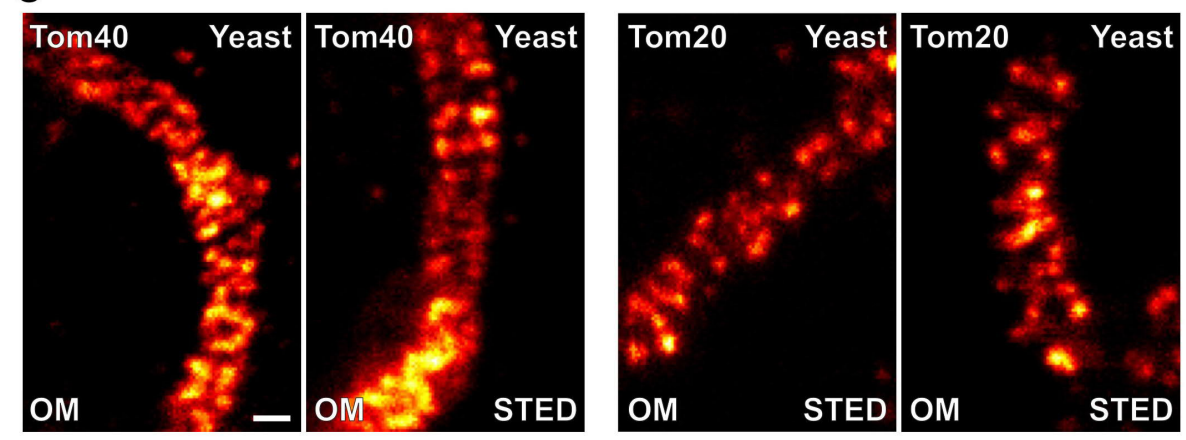
Fig. S4. Nanoscale distribution of yeast mitochondrial inner and outer membrane proteins.

A. Confocal overview images of Mic60-GFP in wild type and $\Delta m i c 10$ cells labeled with a GFP-specific antiserum.

B. Localization of integral membrane proteins of the mitochondrial inner membrane (IM) recorded by single-color STED nanoscopy. Yeast strains expressing GFP-tagged versions of the indicated proteins from their native loci were labeled using a GFP-specific antiserum. Shown are exemplary images.

C. Localization of integral membrane proteins of the mitochondrial outer membrane (OM) recorded by single-color STED nanoscopy. Tom 40 was labeled using a specific antiserum. Yeast strains expressing GFP-tagged versions of the indicated proteins from their native loci were labeled using a GFP-specific antiserum. Shown are exemplary images.

With the exception of contrast stretching no further image processing was applied. Scale bars: $5 \mu \mathrm{m}$ (A), $200 \mathrm{~nm}$ (B, C). 
Table S1. Yeast strains generated for this study

\begin{tabular}{|c|c|c|}
\hline Strain & $\begin{array}{l}\text { Tagged } \\
\text { Protein }\end{array}$ & Genotype \\
\hline Mic60-GFP (W303) & Mic60-GFP & $\begin{array}{l}\text { MATa; ura3-1; trp1A 2; leu2-3, 112; his3-11, 15; ade2-1; } \\
\text { can1-100; MIC60GFP::HIS3 }\end{array}$ \\
\hline $\begin{array}{l}\text { Mic60-GFP; } \Delta \text { mic10 } \\
\text { (W303) }\end{array}$ & $\begin{array}{l}\text { Mic10-K.O.; } \\
\text { Mic60-GFP }\end{array}$ & $\begin{array}{l}\text { MATa; ura3-1; trp1D 2; leu2-3, 112; his3-11, 15; ade2-1; } \\
\text { can1-100; MIC60GFP::HIS3; mic10::kanMX }\end{array}$ \\
\hline Mic27-GFP (W303) & Mic27-GFP & $\begin{array}{l}\text { MATa; ura3-1; trp1D 2; leu2-3, 112; his3-11, 15; ade2-1; } \\
\text { can1-100; MIC27GFP::HIS3 }\end{array}$ \\
\hline Mic12-GFP (W303) & Mic12_GFP & $\begin{array}{l}\text { MATa; ura3-1; trp1D 2; leu2-3, 112; his3-11, 15; ade2-1; } \\
\text { can1-100; MIC60GFP::HIS3 }\end{array}$ \\
\hline Tom20-GFP (W303) & Tom20-GFP & $\begin{array}{l}\text { MATa; ura3-1; trp1D 2; leu2-3, 112; his3-11, 15; ade2-1; } \\
\text { can1-100; TOM2OGFP::HIS3 }\end{array}$ \\
\hline Om14-GFP (W303) & Om14-GFP & $\begin{array}{l}\text { MATa; ura3-1; trp1D 2; leu2-3, 112; his3-11, 15; ade2-1; } \\
\text { can1-100; OM14GFP::HIS3 }\end{array}$ \\
\hline
\end{tabular}


Table S2. Primer for genomic tagging

\begin{tabular}{|c|c|c|}
\hline $\begin{array}{l}\text { Gene/ } \\
\text { ORF }\end{array}$ & $\begin{array}{l}\text { Primer } \\
\text { name }\end{array}$ & Primer sequence \\
\hline MIC60 & Mic60_S3 & $\begin{array}{l}\text { GAAGTCCAGCGCCTGGTAGAGATTCTTGACTGTGAAATAAGGACGTTGCGTACGC } \\
\text { TGCAGGTCGAC }\end{array}$ \\
\hline MIC60 & Mic60_S2 & $\begin{array}{l}\text { GGAAATTGAGGTGTAATGACGTACATCTCTTITCTCTTTGTATTATTCTTTCAATCG } \\
\text { ATGAATTCGAGCTCG }\end{array}$ \\
\hline MIC27 & Mic27_S3 & $\begin{array}{l}\text { CAAAAAAATATAAGGTATGCAAGGGAACAGCTTTATGAAAAGTTGGAGCAAGCA } \\
\text { CGTACGCTGCAGGTCGAC }\end{array}$ \\
\hline MIC27 & Mic27_S2 & $\begin{array}{l}\text { CTTACTATGGACATGATAATGAACAAAAAAAGATATCCGCTTGATATCAATCGATG } \\
\text { AATTCGAGCTCG }\end{array}$ \\
\hline MIC12 & Mic12_S3 & $\begin{array}{l}\text { GAACAGATAAGAAACTCTGTCGATTGGATCTACTCCTGGGGTAAGAATCGTACGC } \\
\text { TGCAGGTCGAC }\end{array}$ \\
\hline MIC12 & Mic12_S2 & $\begin{array}{l}\text { GGTTATATACATGAGGATGTTCGTTACAGTAGGAGAAATAGAAAGCTCGCTAATC } \\
\text { GATGAATTCGAGCTCG }\end{array}$ \\
\hline TOM20 & Tom20_S3 & $\begin{array}{l}\text { GCAAGGCCGAATCTGATGCGGTTGCTGAAGCTAACGATATCGATGACCGTACGCT } \\
\text { GCAGGTCGAC }\end{array}$ \\
\hline TOM20 & Tom20_S2 & $\begin{array}{l}\text { GAGTAAAAGAAACAAAAACGGAGAAAAAAAGCAAGCAAAATGTTACTCTCAATC } \\
\text { GATGAATTCGAGCTCG }\end{array}$ \\
\hline OM14 & Om14_S3 & $\begin{array}{l}\text { CTGGACGGTATAATTTCAAAGAAATACTACTCCAGATACGACAAGAAACGTACGC } \\
\text { TGCAGGTCGAC }\end{array}$ \\
\hline OM14 & Om14_S2 & $\begin{array}{l}\text { GTGAGTGTGTGAGTGTGTGAAAGGATGTTATTAAATAGTATGTTATTAATCGATG } \\
\text { AATTCGAGCTCG }\end{array}$ \\
\hline
\end{tabular}

Table S3. Primer for targeted gene disruption

\begin{tabular}{|l|l|l|}
\hline $\begin{array}{l}\text { Gene/ } \\
\text { ORF }\end{array}$ & $\begin{array}{l}\text { Primer } \\
\text { name }\end{array}$ & Primer sequence \\
\hline MIC10 & Mic10_A & AGCTAGGTGACATGTATACTGGAGC \\
\hline MIC10 & Mic10_D & GGAAGAGAACAAAGTTACAAGTGGA \\
\hline
\end{tabular}


Table S4. Primary antibodies used in this study

\begin{tabular}{|c|c|c|c|c|}
\hline Antibody & $\begin{array}{l}\text { Host } \\
\text { species }\end{array}$ & Depositor/ Supplier & \begin{tabular}{|l|} 
Catalog \\
number
\end{tabular} & Lot number \\
\hline Anti-GFP & Rabbit & Abcam & $a b 6556$ & GR292567-1 \\
\hline $\begin{array}{l}\text { Anti-Porin } \\
\text { [16G9E6BC4] }\end{array}$ & Mouse & MitoSciences (Abcam) & $\begin{array}{l}\text { MSA08 } \\
\text { (ab110326) }\end{array}$ & FKASHK \\
\hline Anti-Mic10 & Rabbit & $\begin{array}{l}\text { Peter Rehling, Georg- } \\
\text { August-University } \\
\text { Göttingen, Germany }\end{array}$ & - & - \\
\hline Anti-Tom40 & Rabbit & $\begin{array}{l}\text { Peter Rehling, Georg- } \\
\text { August-University } \\
\text { Göttingen, Germany }\end{array}$ & - & - \\
\hline Anti-Mic60 & Rabbit & Proteintech & 10179-1-AP & 2 \\
\hline Anti-Tom20 & Rabbit & $\begin{array}{l}\text { Santa Cruz } \\
\text { Biotechnology }\end{array}$ & sc-11415 & G0811 \\
\hline $\begin{array}{l}\text { Anti-Tom20 } \\
\text { [EPR15581] }\end{array}$ & Rabbit & Abcam & ab186734 & $\begin{array}{l}\text { GR219533- } \\
17\end{array}$ \\
\hline Anti-Tom22 & Mouse & $\begin{array}{l}\text { Miltenyi Biotec, Bergisch } \\
\text { Gladbach, Germany }\end{array}$ & - & - \\
\hline
\end{tabular}


Movie S1. Cristae are often arranged in a helical pattern in budding yeast mitochondria. FIB-SEM recording of a budding yeast cell grown in galactose containing medium. Displayed is an orthoslice moving through the data stack recorded by FIB-SEM. The cristae are rendered in blue, green and white, the outer membrane is displayed in gray.

Movie S2. 3D distribution of Mic60 in human mitochondria. Visualization of a 3D STED recording of Mic60 in human A-498 cells. Mic60 was labeled with a specific antiserum against Mic60. 3D STED data were deconvolved, contrast stretched and visualized as a $3 \mathrm{D}$ volume rendition. 


\section{References for SI reference citations}

1. Schauss AC, Bewersdorf J, \& Jakobs S (2006) Fis 1p and Caf4p, but not Mdv1p, determine the polar localization of Dnm1p clusters on the mitochondrial surface. Journal of Cell Science 119(15):3098-3106.

2. Janke C, et al. (2004) A versatile toolbox for PCR-based tagging of yeast genes: new fluorescent proteins, more markers and promoter substitution cassettes. Yeast 21(11):947962. 
Chapter 3 - Mic60 Distribution in Mitochondria

The supplementary movies included in this manuscript can be accessed on the following websites:

Movie S1

Movie S2 


\section{Chapter 4 - Role of MICOS in Cristae Formation}

The following manuscript is under review at The EMBO Journal.

\section{MICOS assembly controls mitochondrial inner membrane remodeling and crista junction redistribution to mediate cristae formation}

Till Stephan ${ }^{1,2, a}$, Christian Brüser ${ }^{1,2, a}$, Markus Deckers ${ }^{3}$, Anna M. Steyer ${ }^{4}$, Francisco Balzarotti ${ }^{1}$, Mariam Barbot ${ }^{1,2}$, Tiana S. Behr ${ }^{1,2}$, Gudrun Heim ${ }^{5}$, Wolfgang Hübner ${ }^{6}$, Peter Ilgen ${ }^{1,2}$, Felix Lange $^{1,2}$, David Pacheu-Grau ${ }^{3}$, Jasmin Pape ${ }^{1}$, Stefan Stoldt ${ }^{1,2}$, Thomas Huser ${ }^{6}$, Stefan W. Hell ${ }^{1}$, Wiebke Möbius ${ }^{4}$, Peter Rehling ${ }^{3}$, Dietmar Riedel ${ }^{5}$ and Stefan Jakobs ${ }^{1,2, b}$

\section{Author affiliations:}

${ }^{1}$ Department of NanoBiophotonics, Max Planck Institute for Biophysical Chemistry, 37077 Göttingen, Germany. ${ }^{2}$ Clinic of Neurology, University Medical Center Göttingen, 37075 Göttingen, Germany. ${ }^{3}$ Department of Cellular Biochemistry, University Medical Center Göttingen, 37073 Göttingen, Germany. ${ }^{4}$ Department of Neurogenetics, Electron Microscopy Core Unit, Max Planck Institute of Experimental Medicine, Göttingen, Germany. ${ }^{5}$ Laboratory of Electron Microscopy, Max Planck Institute for Biophysical Chemistry, 37077 Göttingen, Germany. ${ }^{6}$ Department of Physics, University Bielefeld, 33615 Bielefeld, Germany.

${ }^{\mathrm{a}}$ T.S. and C.B. contributed equally.

${ }^{\mathrm{b}}$ Correspondence should be addressed to S.J. (email: sjakobs@gwdg.de).

Submitted: December 26, 2019; Revised: March 18, 2020 


\section{Author contributions:}

S.J. and T.S. conceived the project. T.S., C.B., M.D., P.R., D.R. and S.J. designed experiments. T.S. and C.B. generated knockout cell lines. T.S. generated stable tetracycline-inducible cell lines and performed knockdown experiments. T.S. and C.B. recorded STED images of knockout mutants. T.S. performed STED nanoscopy of live cells and knockdown cells. J.P. recorded MINFLUX nanoscopy data. W.H., T.S. and S.S. recorded SIM images. D.R., T.S.B, G.H. and F.L. recorded transmission electron microscopy data. A.M.S. recorded FIB-SEM data sets. M.D. and D.P-G. performed respiration measurements. M.D., M.B. and T.S. performed protein biochemistry experiments. T.S., C.B. and S.J. analyzed STED data. J.P., F.B. and S.W.H. analyzed MINFLUX data. W.H. and T.H. analyzed SIM data. T.S. and D.R. analyzed TEM recordings and A.M.S., T.S. and W.M. analyzed FIB-SEM data. M.D., D.P.-G. and P.R. analyzed respiration measurements and protein biochemistry data. T.S. and P.I. segmented EM data sets and created 3D animations. T.S. prepared the manuscript and wrote the paper with S.J.. 


\section{MICOS assembly controls mitochondrial inner membrane remodeling and crista junction redistribution to mediate cristae formation}

Till Stephan ${ }^{1,2, a}$, Christian Brüser ${ }^{1,2, a}$, Markus Deckers ${ }^{3}$, Anna M. Steyer ${ }^{4}$, Francisco Balzarotti ${ }^{1}$, Mariam Barbot ${ }^{1,2}$, Tiana S. Behr ${ }^{1,2}$, Gudrun Heim ${ }^{5}$, Wolfgang Hübner ${ }^{6}$, Peter Ilgen ${ }^{1,2}$, Felix Lange $^{1,2}$, David Pacheu-Grau ${ }^{3}$, Jasmin Pape ${ }^{1}$, Stefan Stoldt ${ }^{1,2}$, Thomas Huser ${ }^{6}$, Stefan W. Hell $^{1,7}$, Wiebke Möbius ${ }^{4}$, Peter Rehling ${ }^{3,7}$, Dietmar Riedel ${ }^{5}$ and Stefan Jakobs ${ }^{1,2,7, b}$

${ }^{1}$ Department of NanoBiophotonics, Max Planck Institute for Biophysical Chemistry, 37077 Göttingen, Germany.

${ }^{2}$ Clinic of Neurology, University Medical Center Göttingen, 37075 Göttingen, Germany.

3 Department of Cellular Biochemistry, University Medical Center Göttingen, 37073 Göttingen, Germany.

${ }^{4}$ Department of Neurogenetics, Electron Microscopy Core Unit, Max Planck Institute of Experimental Medicine, Göttingen, Germany

${ }^{5}$ Laboratory of Electron Microscopy, Max Planck Institute for Biophysical Chemistry, 37077 Göttingen, Germany.

${ }^{6}$ Department of Physics, University Bielefeld, 33615 Bielefeld, Germany

${ }^{7}$ Cluster of Excellence "Multiscale Bioimaging: from Molecular Machines to Networks of Excitable Cells" (MBExC), University of Goettingen, Germany

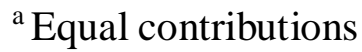

${ }^{\text {b }}$ To whom correspondence should be addressed (email: sjakobs@gwdg.de)
}

Keywords:

Cristae biogenesis; cristae maintenance; electron microscopy; nanoscopy; super-resolution microscopy 


\section{Summary}

Mitochondrial function is critically dependent on the folding of the mitochondrial inner membrane into cristae; indeed numerous human diseases are associated with aberrant cristae morphologies. With the MICOS complex, OPA1 and the $\mathrm{F}_{1} \mathrm{~F}_{\mathrm{o}}$-ATP synthase, key players of cristae biogenesis have been identified, yet their interplay is poorly understood. Harnessing super-resolution light and 3D electron microscopy, we dissect the roles of these proteins in the formation of cristae in human mitochondria. We individually disrupted the genes of all seven MICOS subunits in human cells and re-expressed Mic10 or Mic60 in the respective knockout cell line. We demonstrate that assembly of the MICOS complex triggers remodeling of preexisting unstructured cristae and de novo formation of crista junctions (CJs) on existing cristae. We show that the Mic60-subcomplex is sufficient for $\mathrm{CJ}$ formation, whereas the Mic10subcomplex controls lamellar cristae biogenesis. OPA1 stabilizes tubular CJs, and, along with the $\mathrm{F}_{1} \mathrm{~F}_{\mathrm{o}}$-ATP synthase, fine-tunes the positioning of the MICOS complex and of the CJs. We propose a new model of cristae formation, involving the coordinated remodeling of an unstructured crista precursor into multiple lamellar cristae. 


\section{Introduction}

Mitochondria are essential organelles of eukaryotic cells that perform a multitude of functions. Most notably, they are the powerhouses of the cell that generate ATP through oxidative phosphorylation. Mitochondria feature two membranes, the smooth outer membrane (OM) and the highly convoluted inner membrane (IM). The latter is functionally and structurally divided into two domains, namely the inner boundary membrane (IBM) that parallels the OM and crista membranes (CMs), infoldings of the IM pointing toward the interior of the organelle. The CMs are connected to the IBM by small circular to slit-like openings which are called crista junctions (CJs). The proper folding of the IM is closely related to the function of the organelle and numerous devastating diseases, including cardiomyopathies, neurodegenerative disorders, metabolic diseases, and cancers are associated with aberrant CM folding (Chan, 2012, Friedman \& Nunnari, 2014, Nunnari \& Suomalainen, 2012, Pernas \& Scorrano, 2016, Wai \& Langer, 2016).

In mammalian cells, regularly spaced lamellar cristae appear to be the dominant IM morphology, while also other cristae shapes exist (Cogliati, Enriquez et al., 2016, Hackenbrock, 1966, Scheffler, 2008). Although the basic membrane architecture of mitochondria was discovered almost seven decades ago (Palade, 1952, Sjöstrand, 1953), the molecular mechanisms underlying the formation of cristae are still poorly understood and several models of cristae biogenesis and maintenance have been suggested (Harner, Unger et al., 2016, Muhleip, Joos et al., 2016, Rabl, Soubannier et al., 2009, Zick, Rabl et al., 2009). Recent experimental evidence supports the hypothesis that in budding yeast, lamellar cristae are generated by the conversion of two IBM sheets following a mitochondrial fusion event (Harner et al., 2016). In this study, we show that in human cells, lamellar cristae generation does not require mitochondrial fusion, suggesting fundamental difference in the cristae biogenesis pathways of budding yeast and higher eukaryotes.

Three membrane-shaping factors, namely the dimeric $\mathrm{F}_{1} \mathrm{~F}_{\mathrm{o}}$-ATP synthase, the MItochondrial contact site and Cristae Organizing System (MICOS), and the large GTPase optic atrophy 1 (OPA1, Mgm1 in yeast), exhibit crucial, yet different roles in cristae biogenesis and maintenance. The $\mathrm{F}_{1} \mathrm{~F}_{\mathrm{o}}$-ATP synthase associates into elongated rows of dimers that introduce positive curvature into membranes and stabilize the rims of cristae. Thereby, these dimer rows are important determinants of cristae morphology (Blum, Hahn et al., 2019, Davies, Anselmi et al., 2012, Muhleip et al., 2016, Strauss, Hofhaus et al., 2008). In addition, remodeling of the IM is critically influenced by OPA1 (Alexander, Votruba et al., 2000, Delettre, Lenaers et al., 2000, MacVicar \& Langer, 2016, Varanita, Soriano et al., 2015, Zanna, Ghelli et al., 2008). OPA1 regulates IM fusion (Cipolat, Martins de Brito et al., 2004, Ishihara, Fujita et al., 2006) and presumably fission (Anand, Wai et al., 2014). It also influences the overall cristae architecture and controls the CJ diameter in apoptosis (Frezza, Cipolat et al., 2006, Meeusen, DeVay et al., 2006). Recently, it was shown that Mgm1 from Chaetomium thermophilum can assemble into a helical filament on positively and negatively curved membranes, leading to the 
proposal that Mgm1 might form a helical filament inside of CJs (Faelber, Dietrich et al., 2019). MICOS is a large, hetero-oligomeric protein complex that is primarily located at CJs (Alkhaja, Jans et al., 2012, Harner, Korner et al., 2011, Hoppins, Collins et al., 2011, Rampelt, Zerbes et al., 2017b, von der Malsburg, Muller et al., 2011). In humans, seven MICOS subunits have been identified so far, namely Mic60 (Mitofilin), Mic27 (APOOL), Mic26 (APOO), Mic25 (CHCHD6), Mic19 (CHCHD3), Mic13 (QIL1) and Mic10 (MINOS1) (Pfanner, van der Laan et al., 2014, van der Laan, Horvath et al., 2016). The holo-MICOS complex consists of two distinct subcomplexes (Mic10/13/26/27 and Mic60/19/25) that are named according to the core components Mic10 and Mic60, respectively (Anand, Strecker et al., 2016, Friedman, Mourier et al., 2015, Guarani, McNeill et al., 2015). Both, Mic10 and Mic60 show membrane-shaping activity (Barbot, Jans et al., 2015, Bohnert, Zerbes et al., 2015, Hessenberger, Zerbes et al., 2017, Tarasenko, Barbot et al., 2017). Nevertheless, the exact functions of the two MICOS subcomplexes are unknown. The depletion of several MICOS subunits causes a depletion of CJs and the formation of detached CMs in mitochondria (Harner et al., 2011, Hoppins et al., 2011, von der Malsburg et al., 2011).

Genetic and physical interactions between the $\mathrm{F}_{1} \mathrm{~F}_{\mathrm{o}}$-ATP synthase, OPA1 and MICOS have been demonstrated (Darshi, Mendiola et al., 2011, Eydt, Davies et al., 2017, Janer, Prudent et al., 2016, Quintana-Cabrera, Quirin et al., 2018, Rabl et al., 2009, Rampelt, Bohnert et al., 2017a), but the functional interplay of these three major players involved in cristae development remained largely elusive.

In this study, we used CRISPR/Cas9 genome editing to disrupt individually the genes of all seven MICOS subunits. We demonstrate that the Mic60-subcomplex controls the formation of CJs whereas the Mic10-subcomplex is crucial for the formation of lamellar cristae. Inducible stable cell lines allowed us to follow the restoration of lamellar cristae upon re-expression of MICOS proteins. We found that re-formation of the holo-MICOS complex caused extensive remodeling of preexisting aberrant cristae, including also the formation of secondary CJs. We further demonstrate that OPA1, next to stabilizing tubular CJs, influences along with the $\mathrm{F}_{1} \mathrm{~F}_{\mathrm{o}^{-}}$ ATP synthase the positioning of the MICOS complex. Our findings suggest a new model of cristae formation, based on coordinated membrane remodeling of unstructured CMs into highly ordered cristae. 


\section{Results}

\section{HeLa cells feature primarily lamellar cristae}

Live-cell 2D STED nanoscopy of HeLa cells stably expressing cytochrome $c$ oxidase subunit 8A (COX8A) C-terminally fused with a SNAP-tag revealed that these cells predominantly exhibit groups of lamellar cristae spaced by voids that are occupied by mitochondrial nucleoids (Fig 1A) (Stephan, Roesch et al., 2019). To further investigate the folding of the IM in three dimensions we performed focused ion beam milling combined with scanning electron microscopy (FIB-SEM) (Fig 1B; Appendix Fig S1, Movie EV1). Reconstructions of the FIB-SEM data revealed a substantial level of structural heterogeneity of the architecture of the IM. Groups of stacked cristae were often rotated with respect to each other. Occasionally, we observed single cristae oriented perpendicular to the longitudinal axis of the mitochondrion and also a twisted arrangement of the cristae as previously described in mitochondria from yeast (Stoldt, Stephan et al., 2019) and flies (Jiang, Lin et al., 2017) (Movie EV1).

Mic60 has been demonstrated to form clusters that are enriched at CJs (Alkhaja et al., 2012, Harner et al., 2011, Jans, Wurm et al., 2013). In HeLa cells, these clusters often resembled a stripe pattern perpendicular to the longitudinal axis of the mitochondria (Fig EV1A).

A

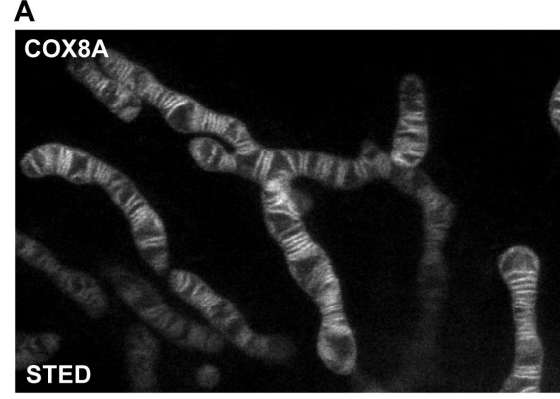

C
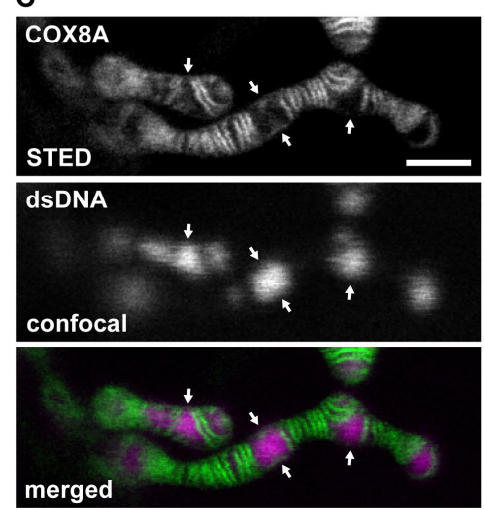

B

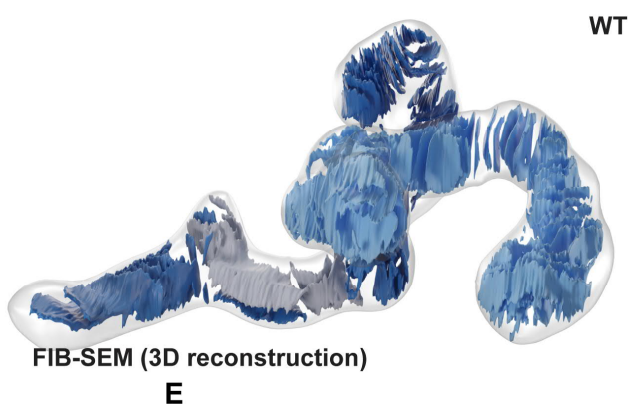

D
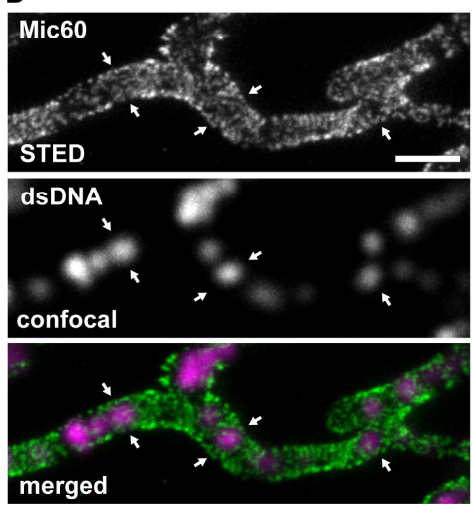

E

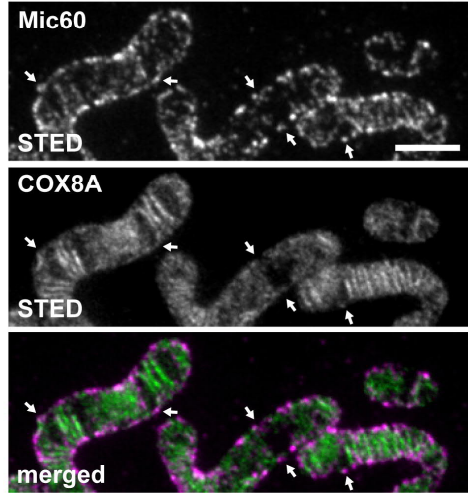

Fig 1. Inner membrane architecture and MICOS distribution in HeLa cells. (A) Live-cell nanoscopy of HeLa cells. COX8A-SNAP was labeled with SNAP-cell SiR and imaged with STED nanoscopy. (B) 3D architecture of cristae in HeLa cells visualized by FIB-SEM. The OM and the IBM are together shown as a transparent surface. Cristae of two mitochondria are depicted in light and dark blue. A twisted crista is shown in grey. (C) Live-cell recording as in (A). In addition, mitochondrial DNA was stained with PicoGreen and imaged by confocal laserscanning microscopy. Arrows mark sites where nucleoids but no lamellar cristae are present. (D) STED nanoscopy of mitochondria immunolabeled for Mic60 and dsDNA. Arrows mark sites where nucleoids are present. (E) Dualcolor STED nanoscopy of cells labeled for COX8A-SNAP and immunolabeled for Mic60. Arrows mark Mic60 clusters in the absence of fully developed lamellar cristae. Scale bars: $1 \mu \mathrm{m}$. 
A
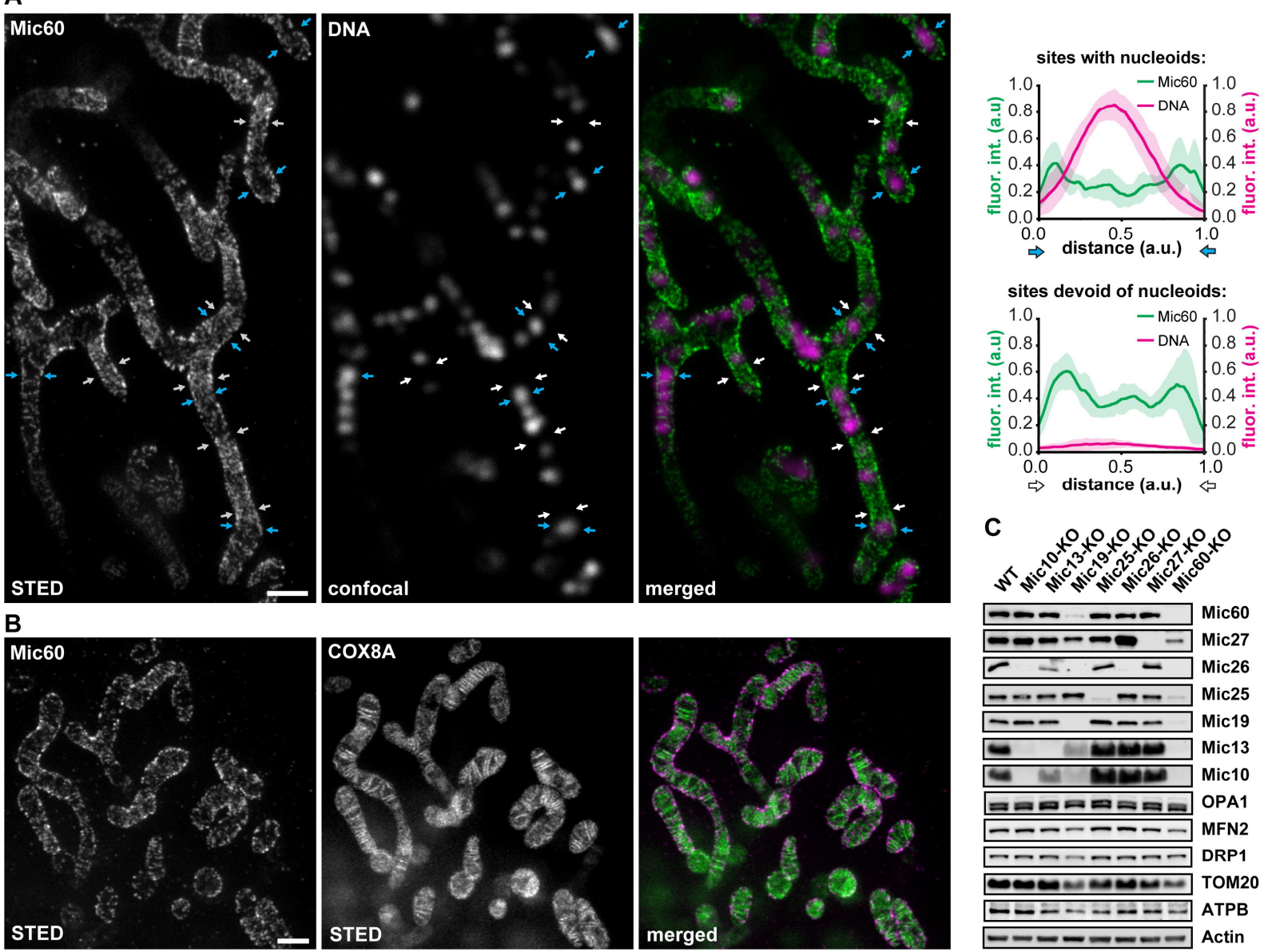

D
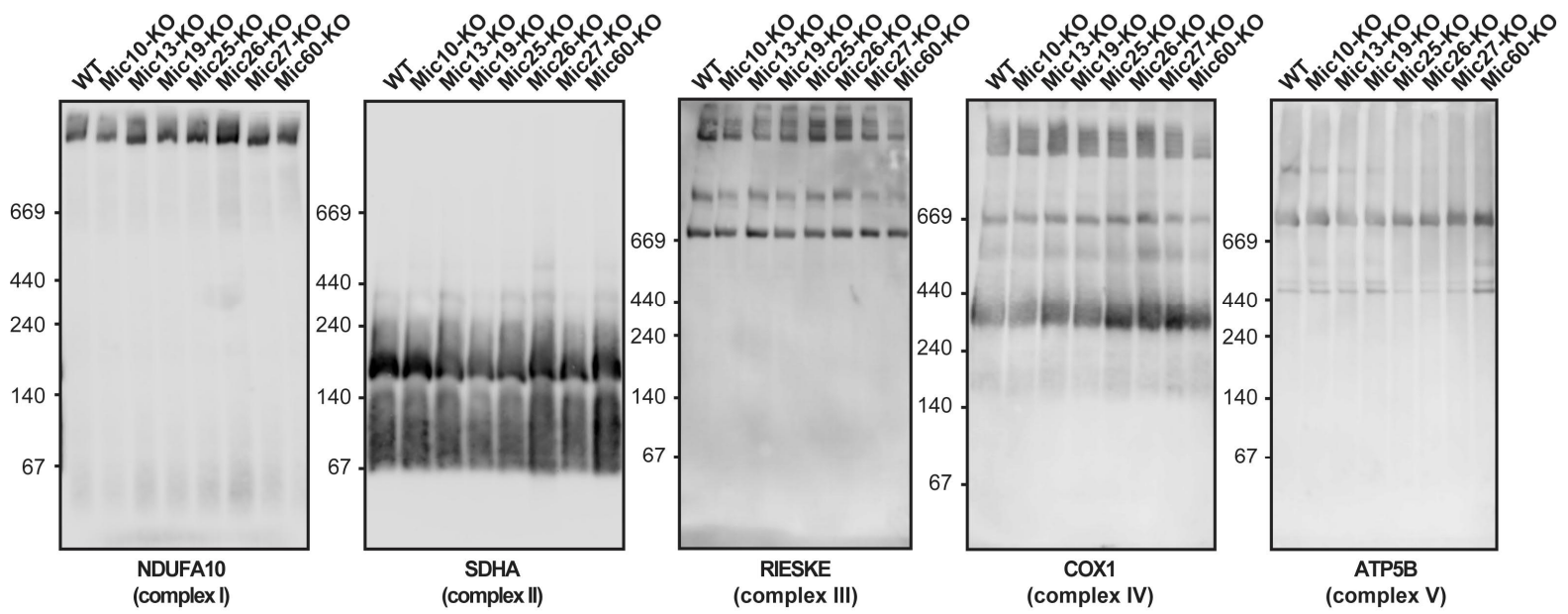

Fig EV1. MICOS distribution in HeLa cells and characterization of MICOS knockout cell lines. (A) Left: STED nanoscopy of mitochondria immunolabeled for Mic60 and dsDNA. A part of the image is also shown in Fig 1D. Right: Line profiles of the fluorescence intensities were measured at six sites exhibiting mtDNA (blue arrows) and at six sites devoid of mtDNA (white arrows), as indicated. The six line profiles of each condition were normalized and averaged, respectively. (B) Dual-color STED nanoscopy of cells labeled for COX8A-SNAP and immunolabeled for Mic60. A part of the image is also shown in Fig 1E. (C) Protein levels of mitochondrial proteins in cell lysates from WT cells and MICOS mutants. Cell lysates from the indicated cell lines were analyzed by Western blotting. (D) BN-PAGE of isolated mitochondria. Indicated cell lines were analyzed by BN-PAGE and Western blotting. Scale bars: $1 \mu \mathrm{m}$. 
Live-cell STED nanoscopy recordings of HeLa cells expressing Mic10-SNAP further verified an arrangement of MICOS clusters in a perpendicular stripe pattern, suggesting that single lamellar cristae can exhibit multiple CJs around a mitochondrion (Movie EV2). Unexpectedly, Mic60 clusters also appeared at sites marked by the mitochondrial nucleoids that are usually free of cristae lamellae (Fig 1C and D, Fig EV1A) (Stephan et al., 2019). In order to verify the occurrence of Mic60 clusters in the absence of proper lamellar cristae, we first labeled cells for COX8A-SNAP and subsequently chemically fixed and immunolabeled them for Mic60. Dualcolor STED recordings confirmed, that while the majority of Mic60 is present at the cristae lamellae, Mic60 clusters also appear in areas devoid of lamellar cristae (Fig 1E, Fig EV1B). 


\section{Characterization of MICOS-KO cell lines}

Depletion of Mic60, but not of Mic10, results in the absence of all MICOS subunits

To dissect the role of the individual MICOS subunits, we disrupted the genes of the seven MICOS proteins (MIC10, MIC13, MIC19, MIC25, MIC26, MIC27 and MIC60) individually in HeLa cells utilizing CRISPR/Cas9 genome editing (for details on the experimental strategy see Appendix Table S1). We analyzed the protein levels of all MICOS subunits in cell lysates and isolated mitochondria from the MICOS-knockout (-KO) cell lines (Fig 2A; Fig EV1C). Deletion of Mic60 was associated with an almost complete loss of Mic10, Mic13, Mic19 and Mic26 and a strong reduction of the Mic25 and Mic27 protein levels (Fig 2A). In Mic19-KO cells, we observed a similar reduction of all MICOS subunits. In contrast, the deletion of Mic10 was associated with a strong reduction of Mic13 and Mic26, whereas the levels of Mic19, Mic25, Mic27 and Mic60 were unaltered. Similarly, the depletion of Mic13 was associated with a decrease in Mic10 and Mic26 levels (Fig 2A). In cell lysates, the levels of other mitochondrial proteins including TOM20 suggested a slightly reduced amount of mitochondria in Mic60-KO and Mic19-KO cells (Fig EV1C), whereas the other KO cell lines were unaffected.

A

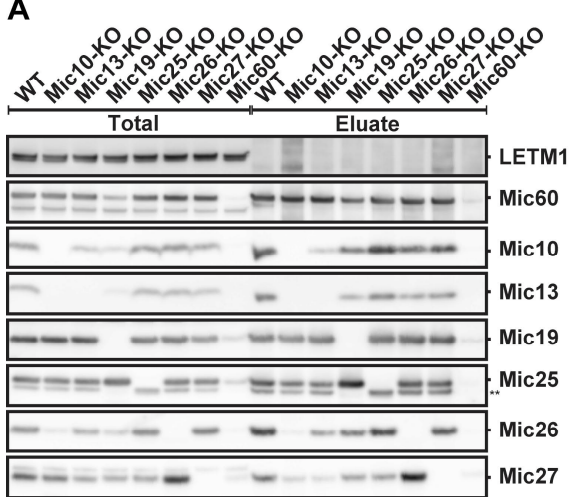

B

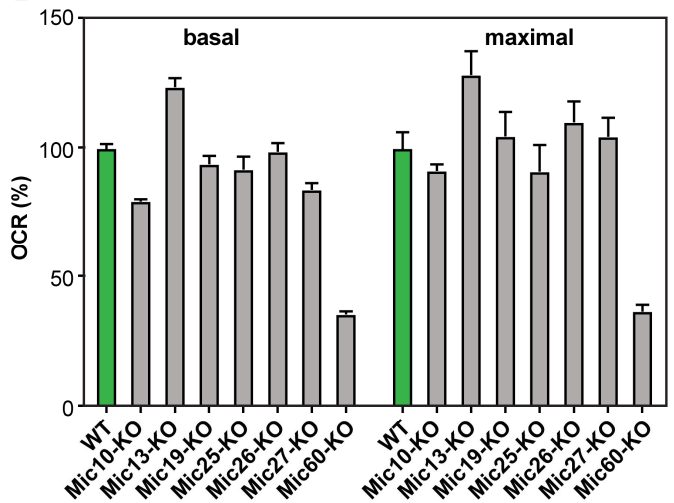

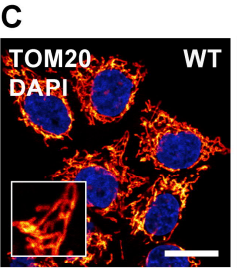
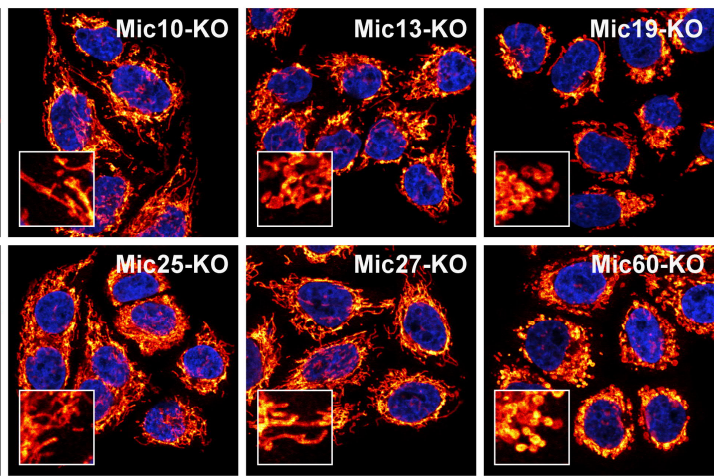

D

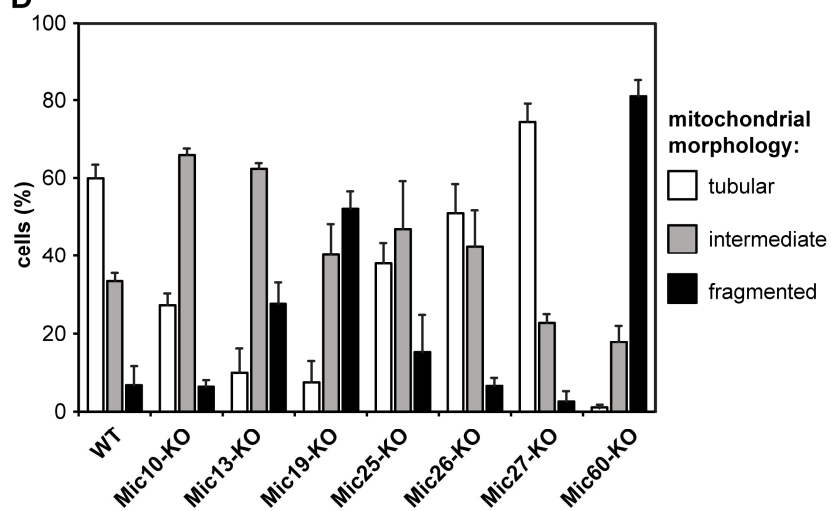

Fig 2. Characterization of MICOS knockout cell lines. (A) Composition of MICOS in WT cells and MICOS mutants. Mic60-specific antibodies were used to pull-down Mic60 and interacting proteins from insolated mitochondria. ${ }^{* *}$ Unspecific band, due to the cross reaction of the anti-Mic25 antibody with Mic19. (B) Oxygen consumption rate (OCR) of WT cells and MICOS mutants as analyzed by Seahorse Analyzer. Basal and maximal OCR are shown relative to the WT. Error bars: SEM, $n=6$. (C, D) Mitochondrial networks of WT and MICOS mutant cells. Cells were immunolabeled for TOM20 and visualized by confocal microscopy. The inset shows a magnification of the respective overview image. Quantification of the mitochondrial networks as shown in (D). Average and SD of 3 independent biological replicates are shown (>170 cells per sample and replicate). Scale bar: $10 \mu \mathrm{m}$. 
As Mic60 controlled the abundance of all other MICOS proteins (Fig 2A, Fig EV1C), we next performed complex-immunoprecipitation (Co-IP) experiments from isolated mitochondria using Mic60 antibodies to investigate whether Mic60 interacts with the remaining MICOS subunits when specific subunits are deleted. In wild-type (WT) cells we isolated Mic10, Mic13, Mic19, Mic25, Mic26 and Mic27, demonstrating a fully assembled holo-MICOS complex (Fig 2A). For the different KO cell lines, the amounts of isolated proteins largely coincided with the steady state levels, suggesting that none of the subunits is essential for the binding of the remaining subunits to Mic60 (Fig 2A). As an exception, we found that in the absence of Mic10 little Mic27 was co-isolated with Mic60, although Mic27 was still present at relatively high levels in these cells.

In line with sequencing data, immunoblotting did not reveal any Mic60 either in whole cell extracts or in isolated mitochondria from Mic60-KO cells (Fig 2A, Fig EV1C, Appendix Table S1). Unexpectedly, after immunoprecipitation with Mic60 antiserum, we detected on Western blots a faint signal for Mic60 and Mic19 in the Mic60-KO cells (Fig 2A). Possibly, an unknown isoform or alternative splicing might account for the residual amount of Mic60 in these cells. As we could not identify any cell line without this residual expression of Mic60, it is currently unclear, if these trace amounts of Mic60 are functionally relevant.

Altogether, we conclude that the depletion of Mic60 leads to an almost entire loss of MICOS. In contrast, the lack of Mic10 causes specifically a depletion of only the Mic10-subcomplex in human cells.

\section{Assembly of OXPHOS is largely unaffected in MICOS-KO cells}

In order to analyze the influence of the loss of MICOS subunits on the assembly of the supercomplexes of the oxidative phosphorylation (OXPHOS) system, we performed blue native polyacrylamide gel electrophoresis (BN-PAGE) of mitochondria isolated from the MICOS-KOs (Fig EV1D). BN-PAGE showed only slight differences between the individual MICOS-KOs and the WT. Even in the absence of Mic60, virtually resulting in the absence of MICOS, the assembly of complexes I, II, and V was only somewhat and the assembly of complexes III and IV was only slightly decreased (Fig EV1D). In Mic60-KO cells, the oxygen consumption rate was reduced, but not abolished; all other MICOS-KO cell lines exhibited oxygen consumption rates close to the WT (Fig 2B). We conclude that the deletion of MICOS subunits has only modest influence on OXPHOS assembly.

Morphology of the mitochondrial network primarily depends on the Mic60-subcomplex

Next, we analyzed the effects of the depletion of the MICOS subunits on the overall morphology of the mitochondrial network (Fig 2C and D). Mic19-KO and Mic60-KO cells showed the most severe phenotype with highly fragmented mitochondrial networks and large spherical mitochondria. Cells lacking Mic10, Mic13, Mic25 and Mic26 displayed on average only moderately fragmented mitochondrial networks, whereas the Mic27-KO cells exhibited slightly hyperfused mitochondrial networks (Fig 2 C and D). These findings demonstrate that the 
Mic60-subcomplex, but not the Mic10-subcomplex, is crucial to maintain the mitochondrial network structure.

\section{Loss of Mic10, Mic13, Mic19 and Mic60 disturbs cristae architecture}

To analyze the cristae morphology of the seven MICOS-KO cell lines, we first performed transmission electron microscopy (TEM) (Fig 3A, Appendix Fig S2A). For an initial evaluation, we classified the structures of the cristae as 'wild type' (ordered, lamellar cristae) or 'aberrant' (disordered cristae) (Fig 3A and B). In Mic60-KO cells, virtually all mitochondria had an aberrant cristae morphology. Similarly, the Mic10-, 13- and Mic19-KO cells showed a severe phenotype, as more than $75 \%$ of the mitochondria had aberrant cristae. The mitochondria of the Mic25-, Mic26- and Mic27-KO cells exhibited only a mild phenotype with more than $75 \%$ of the mitochondria showing ordered lamellar cristae (Fig 3A and B). Additionally, RNA interference (RNAi) experiments independently confirmed these phenotypes (Appendix Fig S2B and C). To further characterize the cristae shapes in the Mic10-, Mic13-, Mic19-, and Mic60$\mathrm{KO}$ cells, we assigned the cristae phenotypes to more detailed categories (Fig 3C). In Mic10 and Mic13-KO cells, we mostly observed a large single CM that paralleled the IBM (Fig 3 A and C). This phenotype was less frequent in mitochondria from Mic60- or Mic19-KO cells, which instead regularly showed multiple layers of CMs arranged in stacks or onion-like arrangements

(Fig 3A and C, Appendix Fig S2A).

\section{Tubular CJs exist in Mic10-KO cells but not in Mic60-KO cells}

We estimated the number of CJs in each of the seven KO strains on several hundred TEM cross sections through mitochondrial tubules. For a comparison of the different cell lines, the number of CJs was normalized to the length of the OM (Fig 3D). Compared to WT cells, the occurrence of CJs was reduced by about $25 \%$ in Mic26-KO cells und by more than $70 \%$ in Mic10-, Mic13and Mic19-KO cells. In Mic60-KO cells, the number of CJs was close to zero. We note, however, that in Mic60-KO cells, we occasionally observed connections of the IBM with the CM (Fig 3D). WT cells, as well as Mic13-, Mic19-, Mic25-, Mic26- and Mic27-KO cells had CJs with an average diameter of about $20 \mathrm{~nm}$ (Fig 3E). In Mic10-KO cells the average diameter was enlarged to about $28 \mathrm{~nm}(\mathrm{n}=96)$ and for the few unusually shaped connections found in Mic60-KO cells ( $\mathrm{n}=26)$ the average diameter was about $32 \mathrm{~nm}$ (Fig 3E).

Compared to previous findings in yeast (Barbot et al., 2015, Bohnert et al., 2015, Harner et al., 2011), the presence of a substantial number of CJs (about $20 \%$ of the WT) in Mic10-KO cells was unexpected. To further analyze this finding, we performed electron tomography (ET) of WT, Mic10-KO and Mic60-KO cells (Fig 3F). Recordings of tilt series thereby allowed us to analyze and reconstruct the CMs and CJs within mitochondrial sections of approximately $200 \mathrm{~nm}$ thickness. 
A
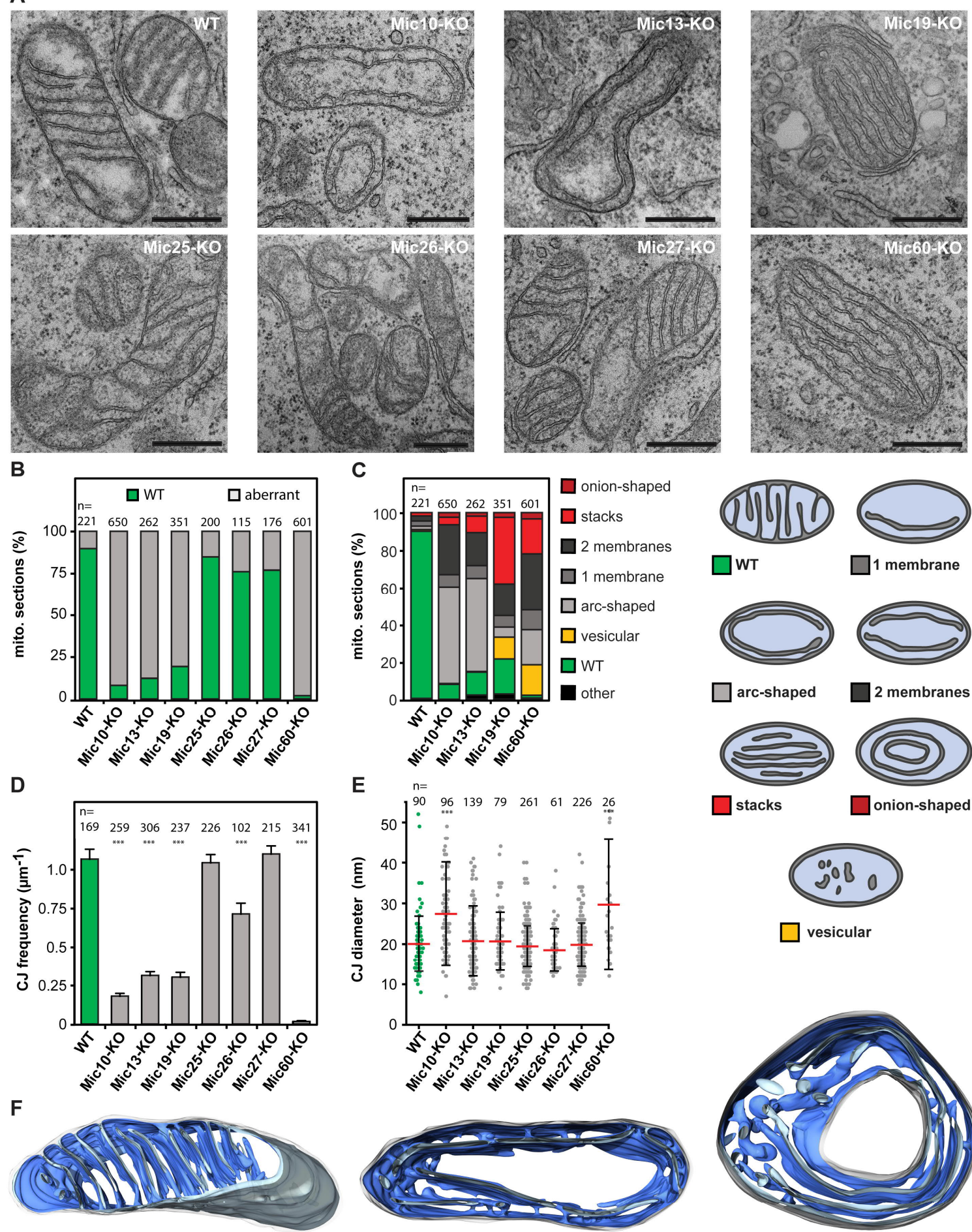

$\square$ wT

1 membrane
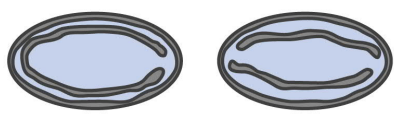

$\square$ arc-shaped

2 membranes
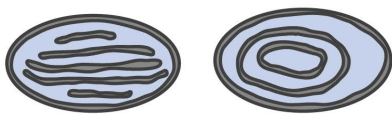

stacks

onion-shaped

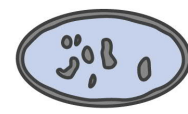

$\square$ vesicula
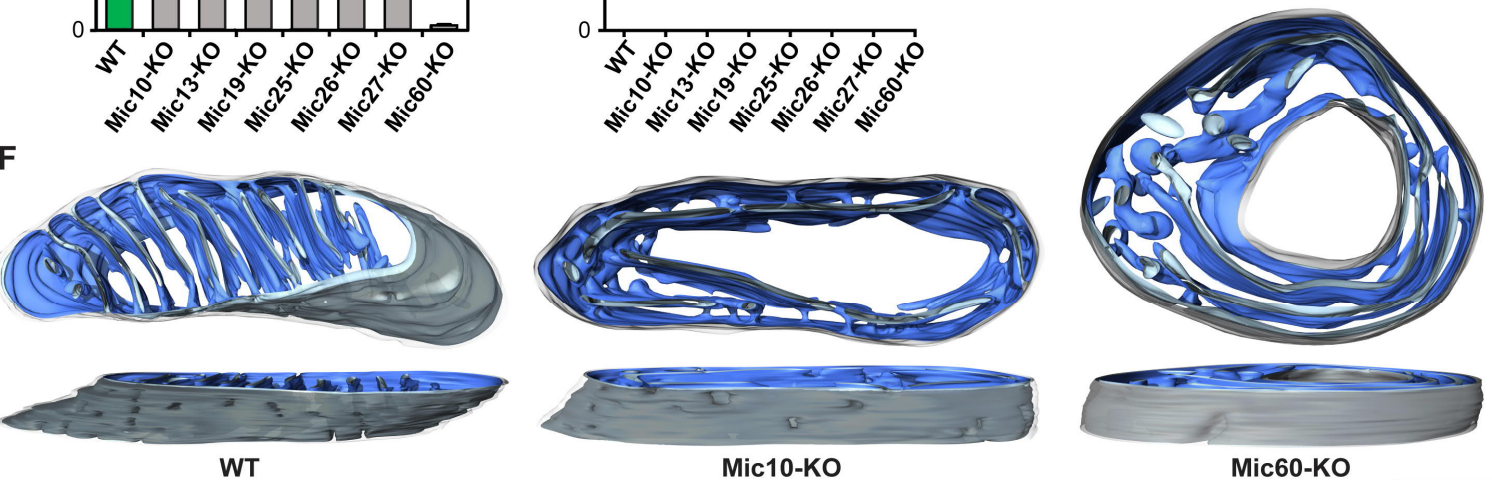

Fig 3. Depletion of MICOS subunits affects the formation of lamellar cristae. (A) Representative TEM recordings of WT cells and MICOS-KO mutants, as indicated. (B) Quantification of the overall cristae morphology on TEM recordings. (C) Quantification of detailed morphology of CMs in MICOS mutants (left). TEM recordings were assigned to 8 classes (right). (D) CJ frequency on TEM recordings. The number of CJs was manually determined and normalized to the length of the OM. Samples were compared to WT by a One-way ANOVA test. Error bars: SEM. (E) Diameter of CJs. CJs were manually measured on TEM recordings. Red line: Average. Error bars: SD. Samples were compared to WT by a One-way ANOVA test. (F) Representative ET reconstructions of mitochondria from a WT cell (left), a Mic10-KO cell (center) and a Mic60-KO cell (right). The OM is displayed in clear grey, the side of the IM that faces the matrix is shown in dark blue. The IM side that faces the IMS is shown in light blue. $n$ : number of mitochondrial sections $(B, C, E)$ or number of CJs (D). ${ }^{* * *}: p \leq 0.001$. Scale bars: $500 \mathrm{~nm}$ (A), $250 \mathrm{~nm}(\mathrm{~F})$. 
Tomograms of Mic60-KO mitochondria confirmed that the CMs frequently formed multilayered, very long sheets, resulting in an onion-like architecture. As expected from the TEM recordings of ultrathin sections, we found only very few CJs in the tomograms (Fig $\mathbf{3 F}$, Movie EV3 and EV4). Vesicular structures in the Mic60-KO mitochondria usually proved to be tubular extensions of large sheet-like cristae (Fig 3F, Movie EV3). The tomograms also confirmed that the lamellar cristae of WT cells exhibited numerous circular or slit-like CJs (Fig 3F; Movie EV5). CJs connected to the same single crista were often close to each other and thereby they formed a line pattern perpendicular to the longitudinal axis of the mitochondrial tubule (Fig $\mathbf{3 F}$; Movie EV5). As Mic60 is enriched at CJs (Jans et al., 2013), the distribution of the CJs explains the perpendicular stripe pattern seen in STED images of Mi60 and Mic10 in these cells (Fig 1D, Fig EV1A). The tomograms showed that in mitochondria of Mic10-KO cells, the cristae usually formed single-layered large sheets with circular, stalk-like tubular CJs (Fig 3F, Movie EV6). In the rare Mic10-KO mitochondria with onion-shaped IM morphology (Movie EV7), these CJs connected the outermost crista membrane with the IBM (Fig 3F, Movie EV7). The stalk-like CJs found in Mic10-KO cells were structurally different from those observed in WT cells, which were circular or slit-like and immediately continuous with the lamellar cristae. Furthermore, in Mic10-KO cells, the CJs appeared to be irregularly distributed and, compared to the WT, often confined to small areas.

Taken together, Mic10-, Mic13-, Mic19- and Mic60-KO cells show a strongly altered cristae architecture. Remarkably, human mitochondria depleted of Mic10 still exhibit numerous tubular, stalk-like, slightly enlarged CJs, whereas CJs are nearly absent in Mic60-KO cells. As Mic10-KO cells contain the Mic60-subcomplex, we conclude that the Mic60-subcomplex, but not the Mic10-subcomplex, is necessary for the formation of CJs. 


\section{Mic10 controls the distribution of Mic60 and the formation of Mic60 assemblies}

The irregular distribution of CJs in Mic10-KO cells suggested that in Mic10-KO cells also the Mic60-subcomplexes have an aberrant distribution. To test this, we performed 2D STED nanoscopy. In HeLa WT cells the Mic60 clusters were seemingly distributed across the mitochondria, often resembling a stripe pattern perpendicular to the longitudinal axis of the mitochondria (Fig 4A, Fig EV1A). In mitochondria of Mic10-KO cells we found that Mic60 and Mic19 clusters localized in clearly discernibly opposite distribution bands, i.e. they exhibited a two-sided distribution on the mitochondrial tubules (Fig 4A, Fig EV2A). This opposite distribution of Mic60 clusters was also observed in Mic13-KO cells (Fig EV2B), showing that the Mic10subcomplex influences the distribution of the Mic60-subcomplexes. Unexpectedly, also the $\mathrm{F}_{1} \mathrm{~F}_{\mathrm{o}}$-ATP synthase subunit $\mathrm{b}$ (ATPB) exhibited a similar two-sided distribution in Mic10-KO cells as visualized by 2D and 3D STED nanoscopy (Fig 4A and B). To further analyze the detailed localization of Mic60 in Mic10-KO cells, we visualized Mic60 with 3D MINFLUX nanoscopy (Gwosch, Pape et al., 2020). Thereby, we were able to localize immunolabeled Mic60 with an isotropic localization precision of about $5 \mathrm{~nm}$ in a $\sim 300 \mathrm{~nm}$ thick volume. MINFLUX recordings confirmed the distribution of Mic60 clusters in two narrow opposite distribution bands along the mitochondrion of a Mic10-KO cell (Fig 4C), which is different to their stripelike distribution in WT cells (Fig EV2C).

This raises the question, if the highly ordered distribution of the Mic60-subcomplex and of the $\mathrm{F}_{1} \mathrm{~F}_{\mathrm{o}}$-ATP is just a consequence of the aberrant cristae morphology. Our ET data strongly suggested that in the absence of Mic10, the CMs form a hollow tube. However, our ET data do not provide an ultimate proof for such a hollow tube, as the tomograms do not encompass a full mitochondrial tubule (Fig 3F). We therefore aimed to visualize the fold of the IM in the entire mitochondrial volume using live-cell fluorescence microscopy as well as FIB-SEM. We labeled the cells with Mitotracker Green and imaged the IM using 3D structured illumination microscopy (3D SIM). 3D SIM of Mic10-KO cells fully supported the view that mitochondria devoid of Mic10 contained tube-like cristae that line the interior of the mitochondrion (Fig 4D, Fig EV2D). Live-cell STED recordings of Mic10-KO cells expressing COX8A-SNAP further confirmed that the mitochondria in Mic10-KO cells contain mobile, large tube-like cristae (Movie EV8 and 9). Finally, FIB-SEM of Mic10-KO cells unequivocally demonstrated the presence of tube-like cristae which were partly perforated (Fig $4 \mathrm{E}$ ). Because the edges of the perforations have a positive membrane curvature, we speculate that the $\mathrm{F}_{1} \mathrm{~F}_{\mathrm{O}}$-ATP synthases might decorate the perforations and therefore are located at the rims of the mitochondria.

The ET, FIB-SEM and super-resolution data conclusively demonstrated that in Mic10-KO cells the CMs form generally single-layered hollow tubes. It is difficult to reconcile the formation of the opposite Mic60 distribution bands with a large tube-like CM that evenly lines the mitochondrial tubule. We concluded that the distribution of the $\mathrm{F}_{1} \mathrm{~F}_{\mathrm{o}}$-ATP synthase and of the Mic60 clusters in opposite bands is not primarily determined by the shape of the tube-like CM. Instead, we assumed that that the abundance of Mic10 controls the distribution of Mic60. 
A

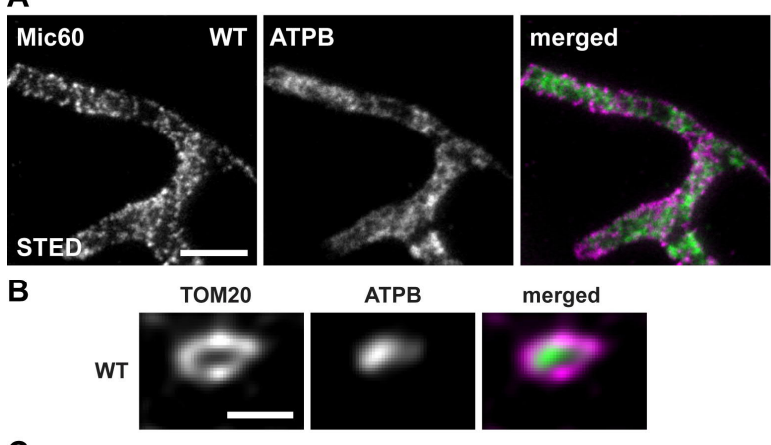

C
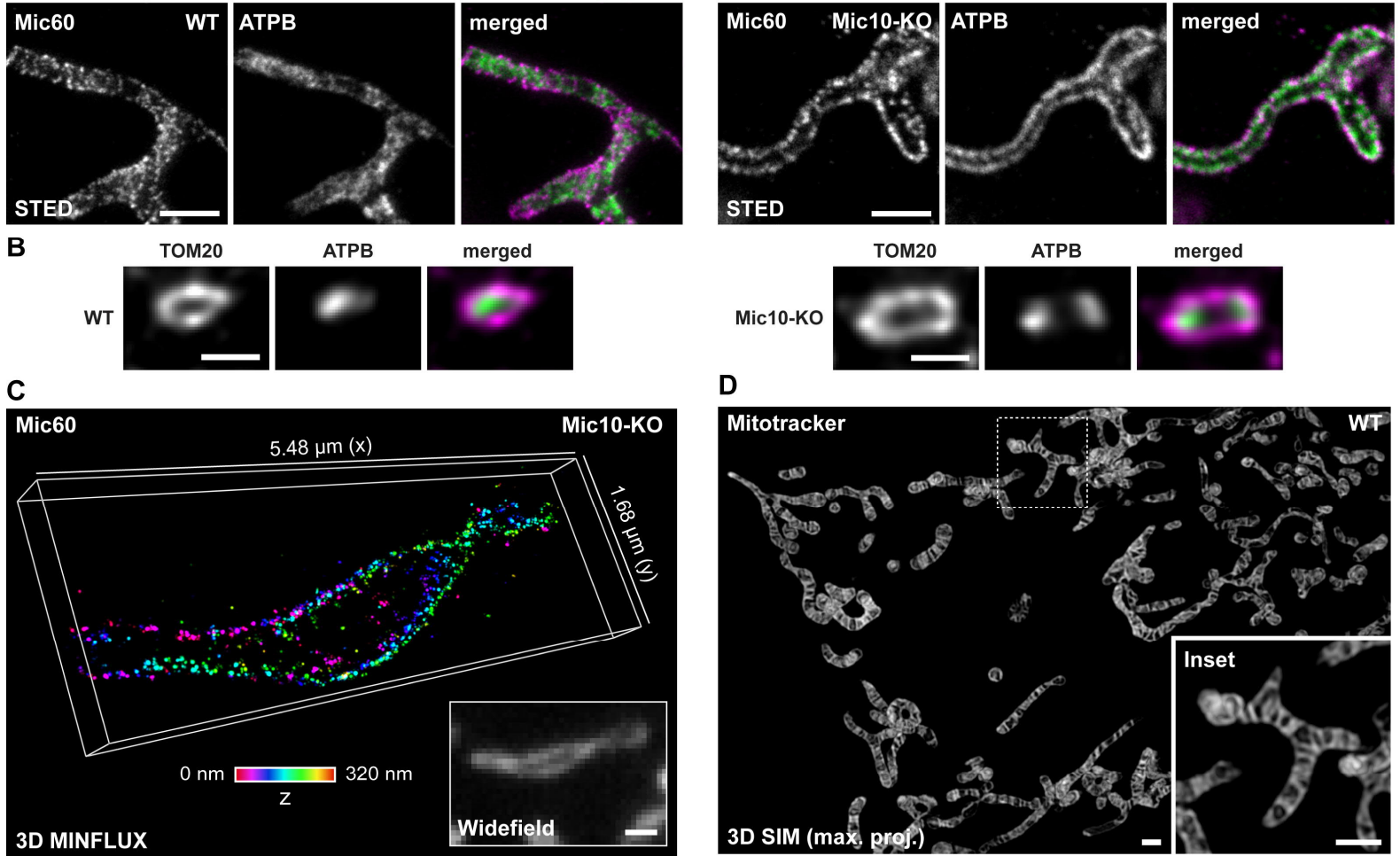

TOM20

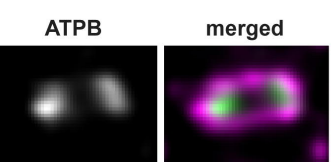

D

E
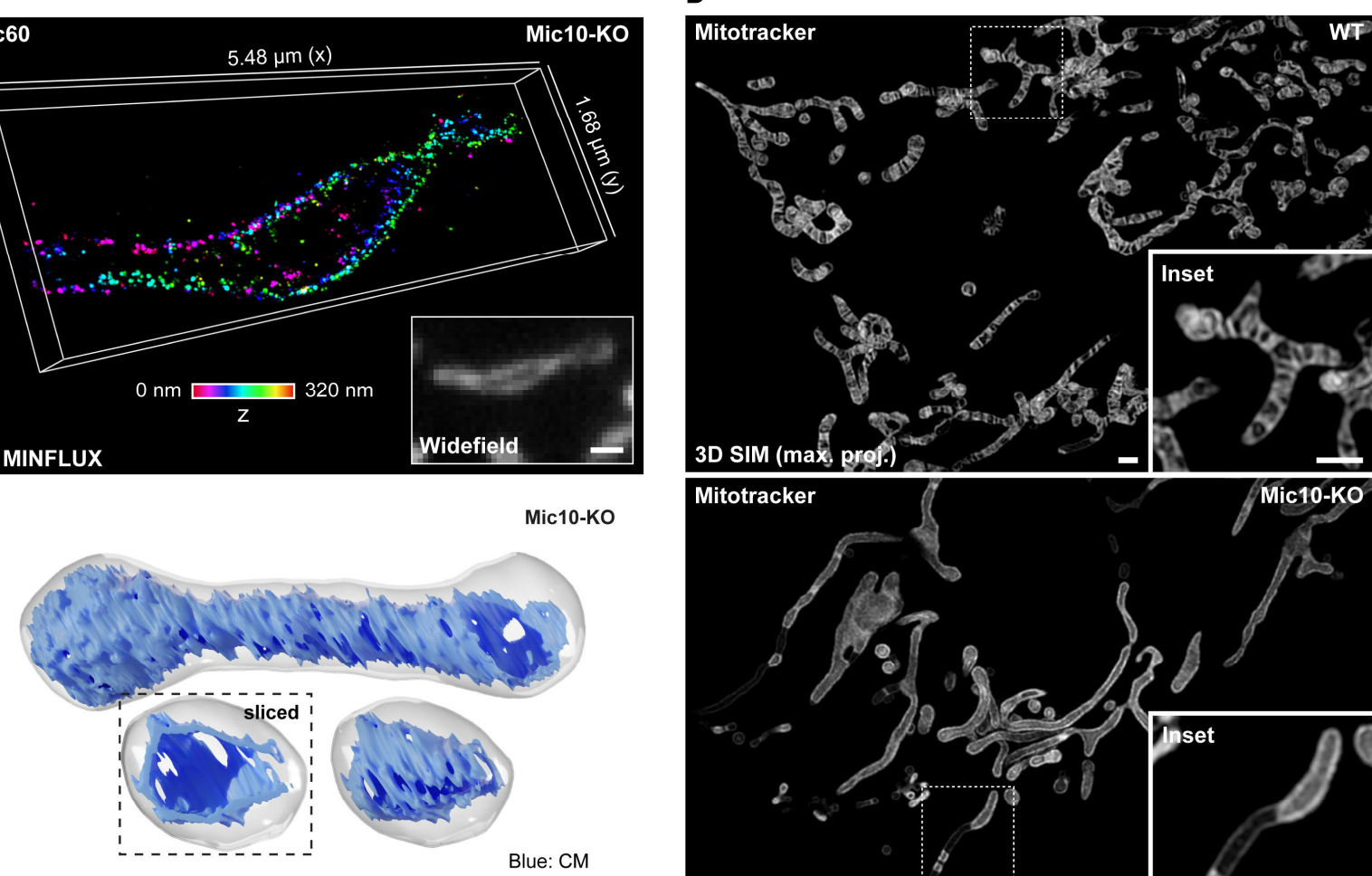

FIB-SEM (3D reconstr.)

Clear: IBM/ OM
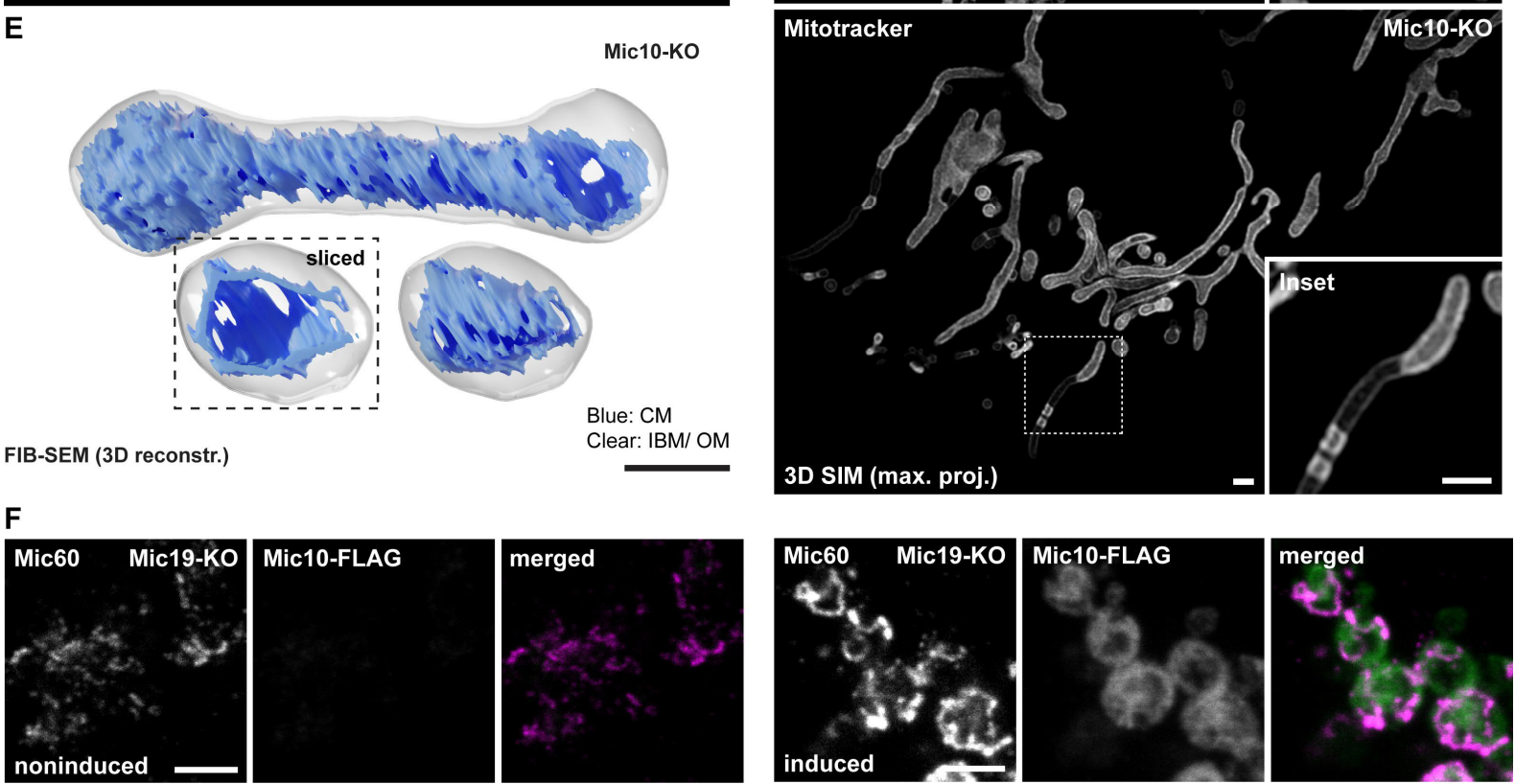

Fig 4. Depletion of Mic10 causes formation of large tube-like cristae and opposite distribution bands of Mic60 and ATPB. (A,B) Nanoscopy of mitochondria of WT and Mic10-KO cells, as indicated. (A) Mitochondria were immunolabeled for Mic60 and ATPB and visualized by 2D STED nanoscopy. (B) Mitochondria were immunolabeled for TOM20 and ATPB and recorded with 3D STED nanoscopy. Image data were deconvolved. Depicted are crosssections of a mitochondrion (side view). (C) 3D MINFLUX nanoscopy. Mic10-KO cells were immunolabeled for Mic60 using a directly labeled antibody. Colors encode depth information. (D) 3D SIM of living WT and Mic10-KO cells. Cells were labeled with Mitotracker Green. Images show maximum intensity projections. (E) FIB-SEM of Mic10-KO cells. Shown is a representative reconstructed mitochondrion. Dashed box: Orthoslice view on the smaller mitochondrion. The OM and IBM are displayed together in clear grey, the CM in is shown in blue. $(F) 2 D$ STED nanoscopy of mitochondria. Mic19-KO cells were transfected with a plasmid for Mic10-FLAG expression under the control of a tetracycline promoter. Left panel: Mitochondria from noninduced cell. Right: Mitochondria from induced cell expressing Mic10-FLAG. Scale bars: $1 \mu \mathrm{m}(A, C, D, F), 500 \mathrm{~nm}(B, E)$. 
A

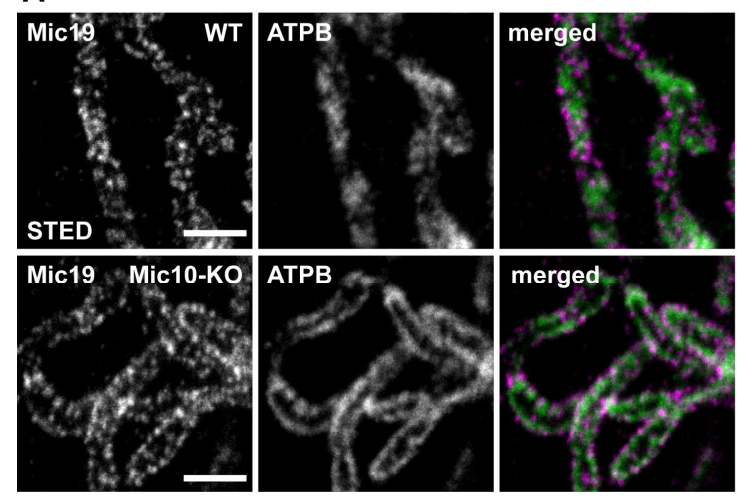

C

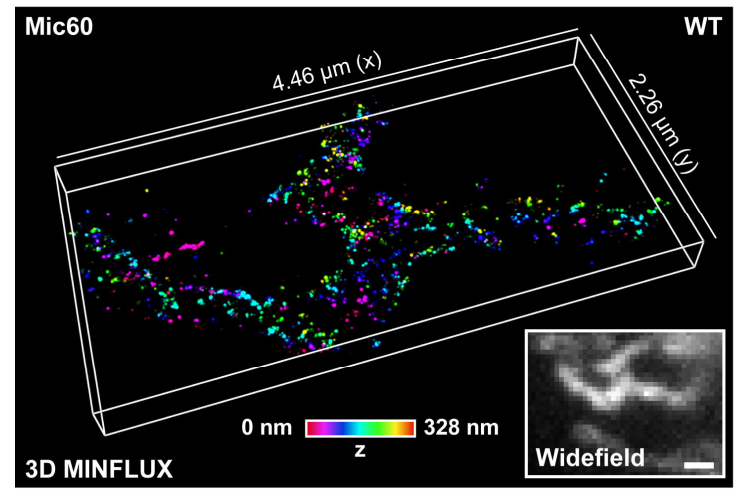

D

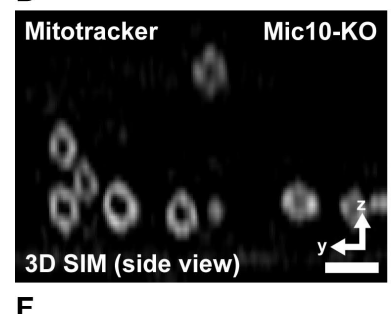

$\mathbf{F}$
B

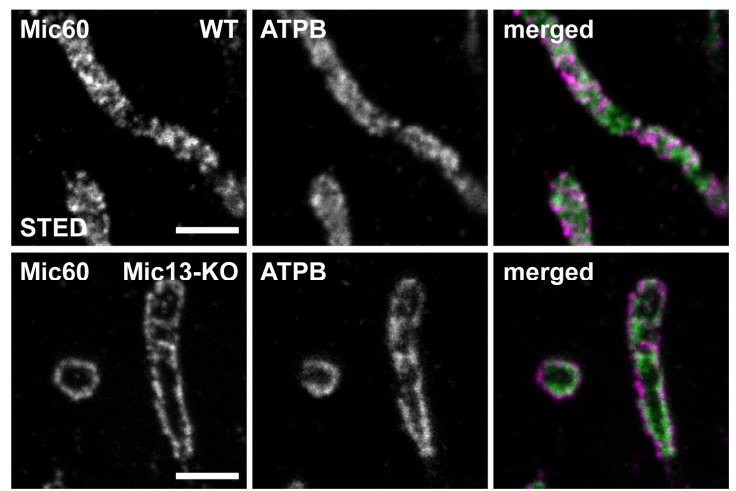

E
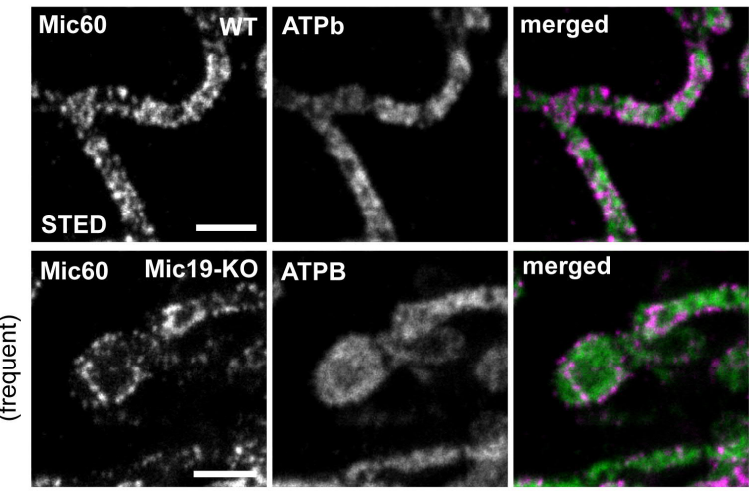

merged

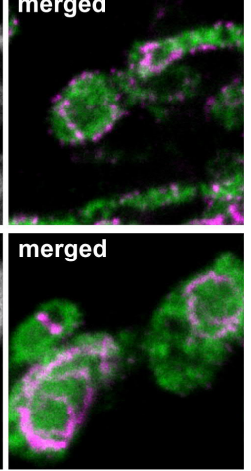

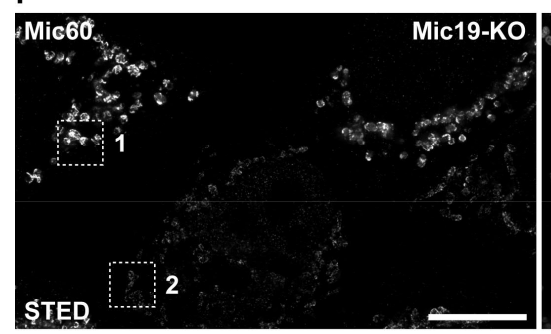
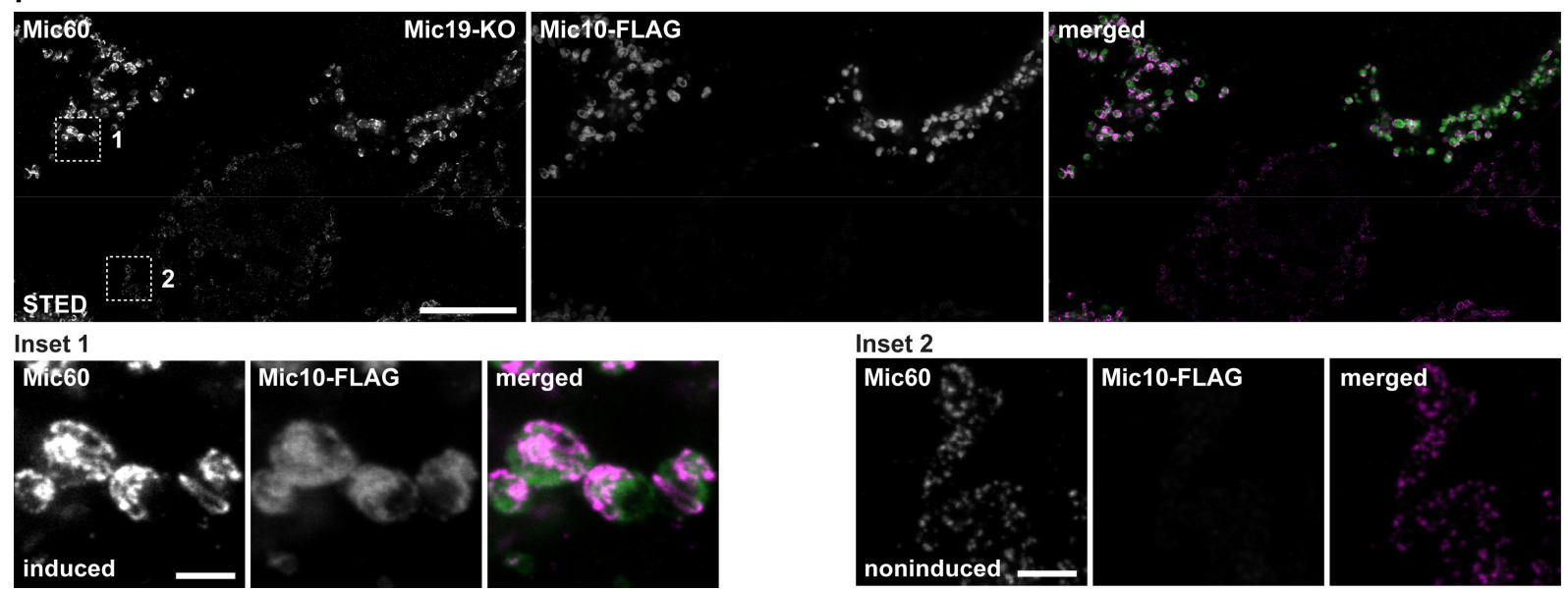

Fig EV2. Depletion of Mic10 causes the formation of large tube-like cristae and opposite distribution bands of Mic60, Mic19 and ATPB. (A-C) Nanoscopy of mitochondria. (A) WT and Mic10-KO cells were immunolabeled for Mic19 and ATPB and visualized by 2D STED nanoscopy. (B) WT and Mic13-KO cells were immunolabeled for Mic60 and ATPB and recorded with 2D STED nanoscopy. (C) 3D MINFLUX nanoscopy of mitochondria. WT cells were immunolabeled for Mic60. Colors encode depth information. (D) 3D SIM of live cells. Mic10-KO cells were labeled with Mitotracker Green. Images show a cross section (side view) through several mitochondria in a 3D SIM stack. (E) STED nanoscopy of mitochondria. WT and Mic19-KO cells were immunolabeled for Mic60 and ATPB and analyzed by STED nanoscopy. (F) Nanoscopy of mitochondria. Mic19-KO cells were transfected with a plasmid for Mic10-FLAG expression under the control of a tetracycline promoter and induced with doxycycline for $24 \mathrm{~h}$. Inset 1: Mitochondria from a cell strongly expressing Mic10-FLAG. Inset 2: Mitochondria from a cell expressing virtually no Mic10-FLAG. Scale bars: $1 \mu \mathrm{m}$ (A-E, and F, lower panel), $10 \mu \mathrm{m}(\mathrm{F}$, upper panel). 
To test this idea, we analyzed the Mic60 distribution in Mic19-KO cells which exhibit reduced Mic60 levels and a strongly aberrant cristae architecture (Fig 3A and B), but remain residual levels of the Mic10-subcomplex (Fig 2A). In these cells, Mic60 was often found in randomly scattered clusters instead of opposite bands (Fig EV2E). In addition, we occasionally observed mitochondria that exhibited continuous ring- or arc-like Mic60 structures (Fig EV2E). The formation of such continuous Mic60 assemblies was strongly increased when we overexpressed Mic10-FLAG in Mic19-KO cells (Fig 4F, Fig EV2F). In addition, Mic10-FLAG overexpression also raised the expression level of Mic60 in Mic19-KOs (Fig EV2F).

Altogether, these data demonstrate that the expression level of Mic10 influences the distribution of Mic60 and also of the $\mathrm{F}_{1} \mathrm{~F}_{\mathrm{o}}$-ATP synthase. In the absence of Mic10, Mic60 is found in clusters localized in opposite distribution bands, whereas at elevated Mic10 levels, Mic60 forms extended assemblies. 


\section{Re-expression of Mic60 in Mic60-KO cells stabilizes MICOS and induces the formation of secondary CJs}

To better understand the role of MICOS in cristae formation, we next analyzed how Mic60deficient mitochondria respond to a rescue of the cellular Mic60 levels. To this end, we inserted the coding sequence of Mic60 under the transcriptional control of a tetracycline-/doxycyclineinducible (TetOn) promoter into the genome of Mic60-KO cells, thereby generating a Mic60TO cell line. After initiation of the re-expression of Mic60 by adding doxycycline, we investigated the effects of increasing Mic60 levels over time (Fig 5). We titrated the concentration of doxycycline to reach approximately endogenous Mic60 levels after $48 \mathrm{~h}$.

A
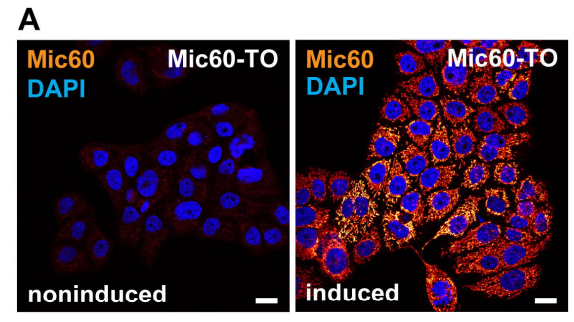

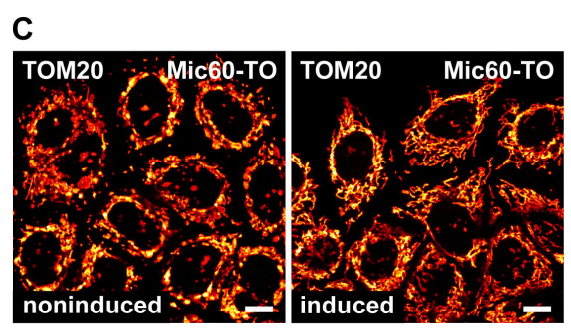

D
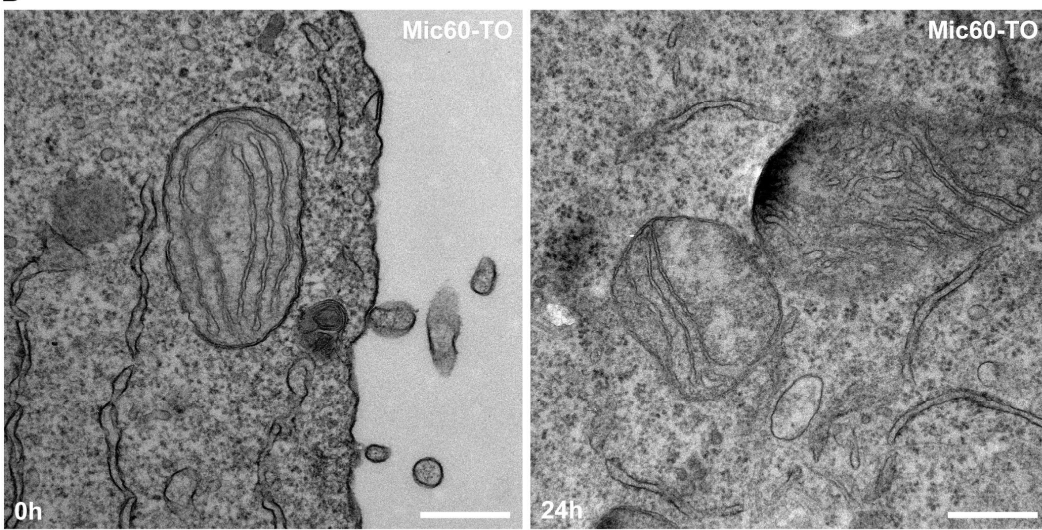

E
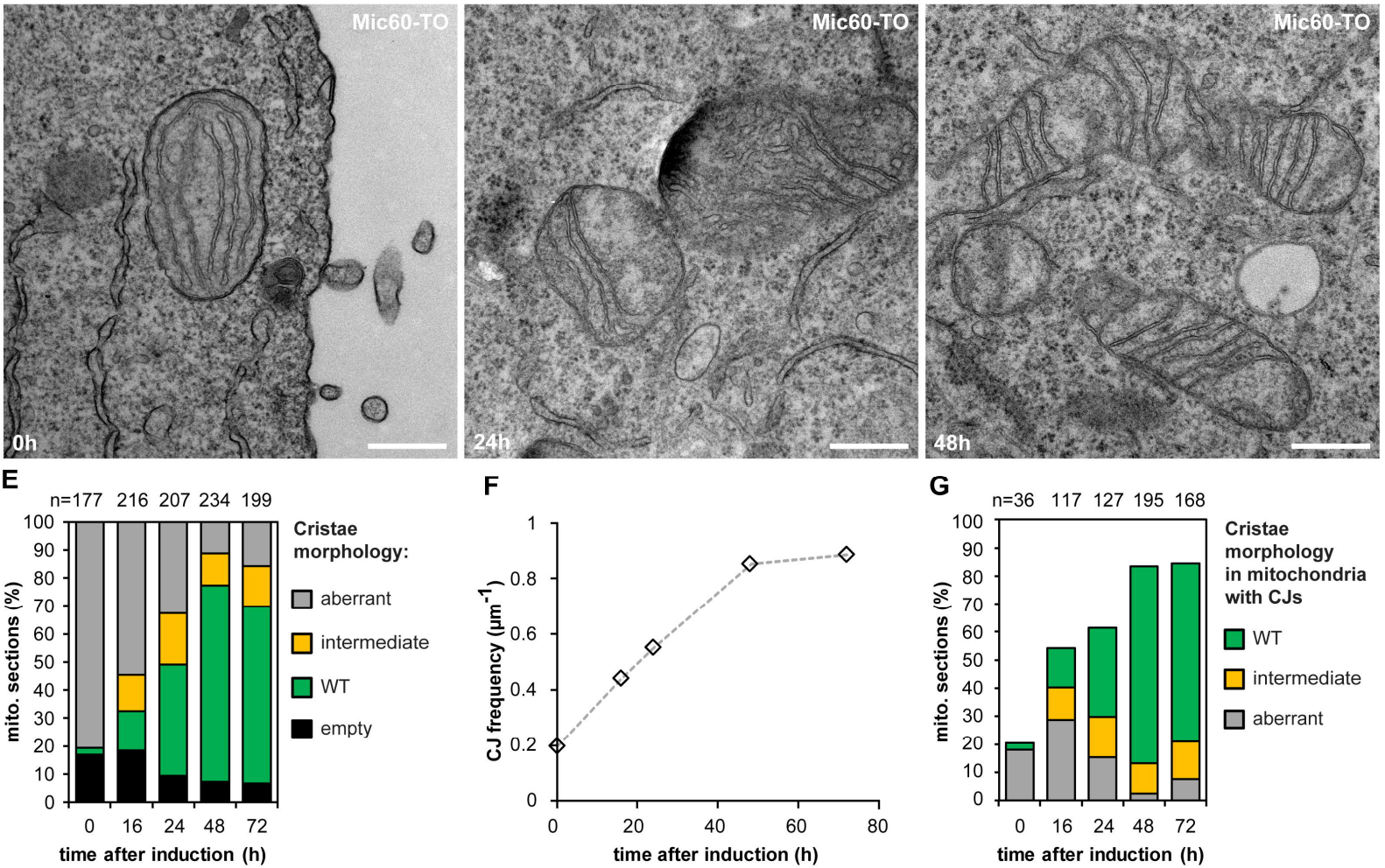

G

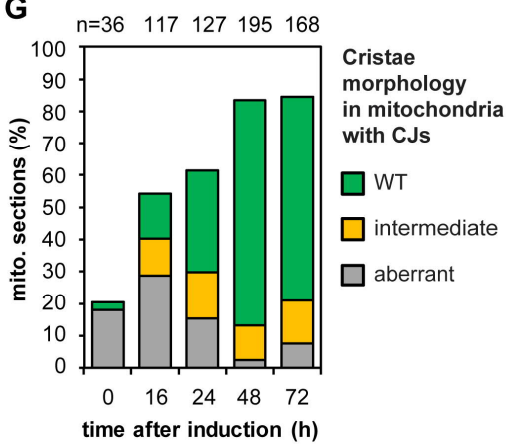

Fig 5. Mic60 controls MICOS protein levels, CJ frequency and inner membrane morphology. Rescue of Mic60 expression in Mic60-TO cells upon induction with doxycycline. (A) Cells were immunolabeled for Mic60 and DNA and visualized by confocal fluorescence microscopy before and after expression of Mic60 for $48 \mathrm{~h}$. (B) Protein levels of MICOS proteins after Mic60 induction. Cell lysates were analyzed by Western blotting at the indicated time points. (C) Recovery of mitochondrial networks upon Mic60 re-expression. Cells were induced with doxycycline for $72 \mathrm{~h}$, immunolabeled for TOM20 and visualized by confocal microscopy. (D-G) TEM of Mic60-TO cells before and after induction. (D) TEM recordings of Mic60-TO cells at the indicated time points. (E) Quantification of the cristae morphology. (F) CJ frequency. The number of CJs on TEM recordings was normalized to the length of the OM. At least 90 mitochondrial sections were analyzed for each sample. (G) Quantification of the cristae morphology of Mic60-TO cells. Only mitochondria exhibiting at least one CJ were analyzed. n: Number of mitochondrial sections. Scale bars: $20 \mu \mathrm{m}(\mathrm{A}), 10 \mu \mathrm{m}(\mathrm{B}), 500 \mathrm{~nm}(\mathrm{C})$. 
After this time, virtually all cells expressed Mic60 (Fig 5A and B, Appendix Fig S3). Mic60 reexpression rescued the cellular protein levels of all MICOS subunits (Fig 5B) and caused the reformation of tubular mitochondrial networks (Fig 5C, Appendix Fig S3B).

Next, we asked whether the aberrant cristae in the Mic60-KO cells are converted to the WT morphology, or whether they are replaced by newly formed cristae. To address this question, we analyzed the morphology changes upon MICOS re-expression over time by analysis of numerous TEM recordings and classified the CM morphologies (Fig 5D and E). We observed a considerable number of mitochondria with intermediately shaped cristae, i.e. IM segments that partly showed a lamellar morphology and partly had an aberrant appearance (Fig 5D and E, Appendix Fig S4). These contiguous IM segments strongly point to a conversion of aberrant cristae. Furthermore, we did not observe an increased number of small cristae that would hint to a strong increase of cristae biogenesis. With rising Mic60 levels, the number of CJs increased steadily over time and reached WT levels after about 48 h (Fig 5F). Importantly, we found that after $16 \mathrm{~h}$ of induction of Mic60 expression, only a fraction of the new CJs were connected to cristae with WT morphology (Fig 5G, Appendix Fig S4). Instead, a substantial part of the CJs induced by Mic60 expression was found on aberrant or intermediately shaped cristae. We conclude that they formed on already existing cristae and denote these CJs as secondary CJs. 


\section{Formation of the holo-MICOS complex results in crista membrane remodeling and a redistribution of the CJs}

The conversion of the cristae in the Mic60-TO cells is difficult to analyze because multiple layers of $\mathrm{CM}$ are involved. In comparison, the Mic10-KO has a less complex IM architecture, as the mitochondria usually exhibit only a single-layered tube-like CM, which is already connected to the IBM by CJs (Fig 3A and F). Therefore, we next aimed to analyze the cristae conversion process upon re-expression of Mic10 in a Mic10-deficient cell line. To this end, we integrated a TetOn version of Mic10 C-terminally fused with a FLAG-T2A-EGFP tag into the genome of Mic10-KO cells. The self-cleaving T2A-peptide (Ryan, King et al., 1991) causes the release of cytosolic EGFP as an expression reporter, whereas the FLAG-tagged Mic10 is transported into the mitochondria (Fig 6A, Fig EV3A). To test whether the re-expression of Mic10FLAG induces the formation of the holo-MICOS complex, we performed Co-IPs from cell lysates using anti-FLAG and anti-Mic60 antibodies (Fig EV3B, Appendix Fig S5A). Using Mic10FLAG as a bait, we pulled down Mic60, Mic26, Mic19 and Mic13 at levels mirroring the increasing Mic10 levels. When we used Mic60 as a bait, we isolated Mic10, Mic13 and Mic26 at levels corresponding to the expression levels of Mic10, whereas Mic19 was isolated irrespective from the Mic10 expression levels (Fig EV3B). These findings demonstrate that reexpression of Mic10-Flag rescues the expression levels of Mic13 and Mic26 and that newly synthesized subunits of the Mic10-subcomplex bind to the pre-existing Mic60-subcomplex to build the fully assembled MICOS complex.

After $48 \mathrm{~h}$ of induction, the large majority of cells expressed Mic10-FLAG and showed WTlike mitochondrial networks (Appendix Fig S5B and C). Moreover, the cristae morphology was rescued in the majority of these cells as demonstrated by live-cell STED nanoscopy (Fig 6A and B). As the expression levels of Mic10 did not rise simultaneously in all cells, few cells still exhibited a low Mic10-expression level after $24 \mathrm{~h}$ of induction, whereas most cells already had an elevated Mic10 expression level. In cells with low Mic10 expression levels, the faint Mic10FLAG signals largely co-localized with Mic60 in narrow opposite distribution bands, comparable to the Mic60 distributions bands observed in Mic10-KO cells (Fig 6C). In accordance with the co-immunoprecipitation experiments (Fig EV3B), this suggests that Mic10FLAG is recruited to the existing Mic60-subcomplexes. In cells showing higher Mic10-FLAG expression levels, Mic10 and Mic60 also largely co-localized, but their distribution in opposite bands was no longer obvious, as the protein clusters spread over the mitochondria (Fig $6 \mathbf{C}$ ). Together, these data suggest that Mic10, after being recruited to the Mic60-subcomplex, changes the distribution of the holo-MICOS complex in the IM.

Next, we recorded TEM images of mitochondria at different time points after re-expression of Mic10-FLAG in Mic10-TO cells and analyzed the process of the structural rescue of the cristae (Fig 6D and E). After $24 \mathrm{~h}$ of induction, the cristae morphology, the number of CJs (Fig 6F), as well as the average $\mathbf{C J}$ diameter (Fig $\mathbf{6 G}$ ) resembled the situation in WT cells. The TEM recordings also revealed that the rescue of the cristae structure seemed to involve an 
'intermediate' phenotype, i.e. contiguous cristae that were partly lamellar and partly tube-like (Fig 6D, inset, Appendix Fig S5D).

A

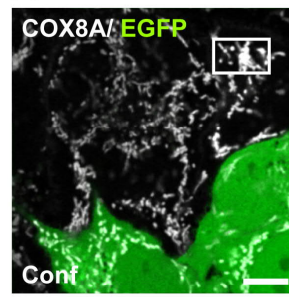

B

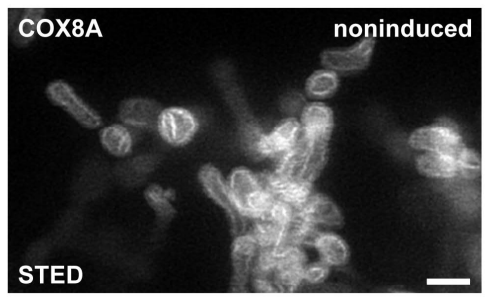

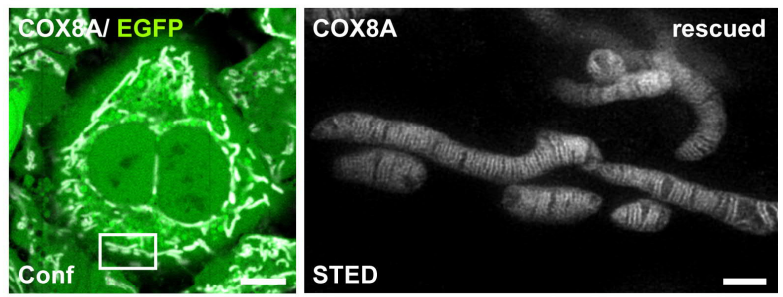

C
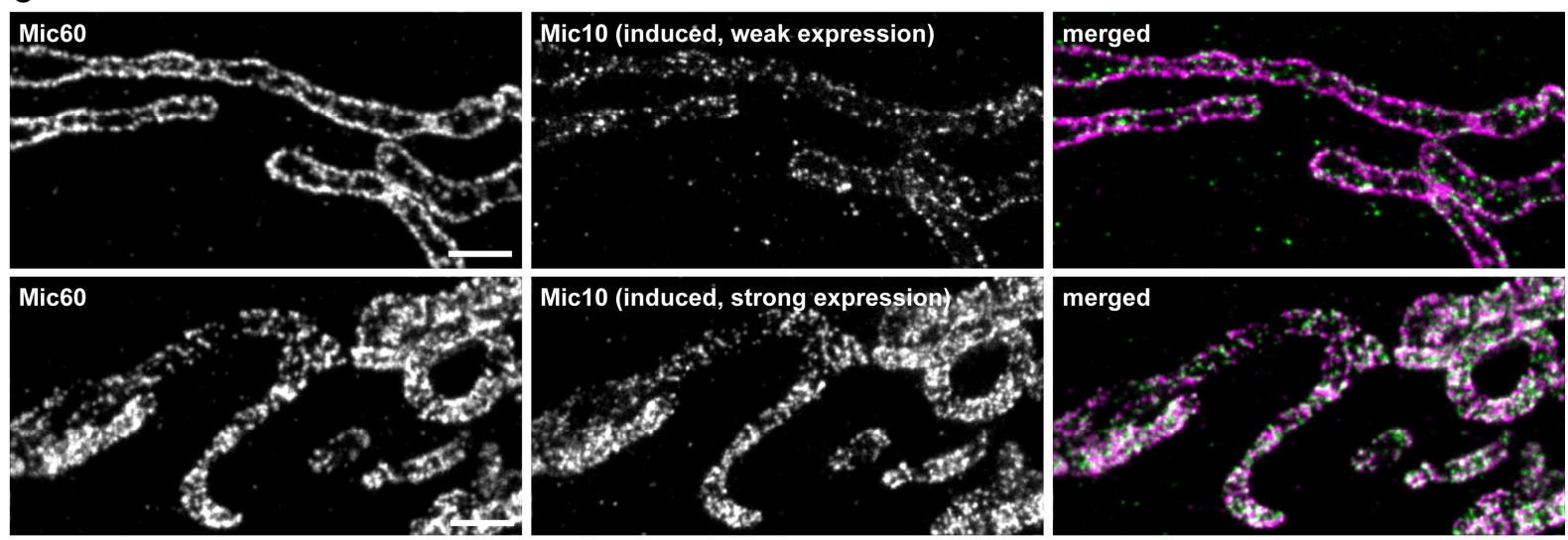

D
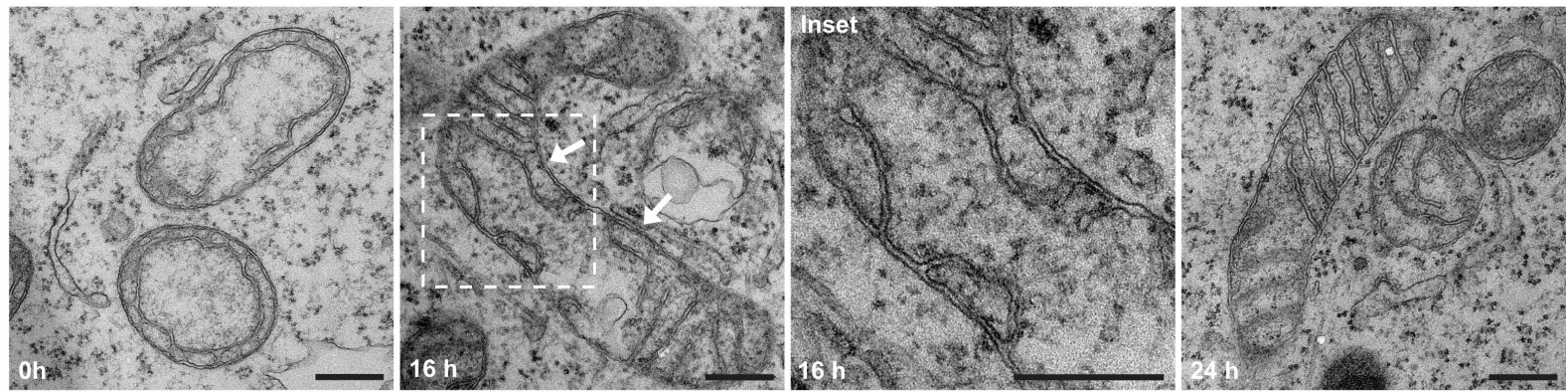

E $n=112 \quad 92 \quad 65 \quad 103 \quad 67$
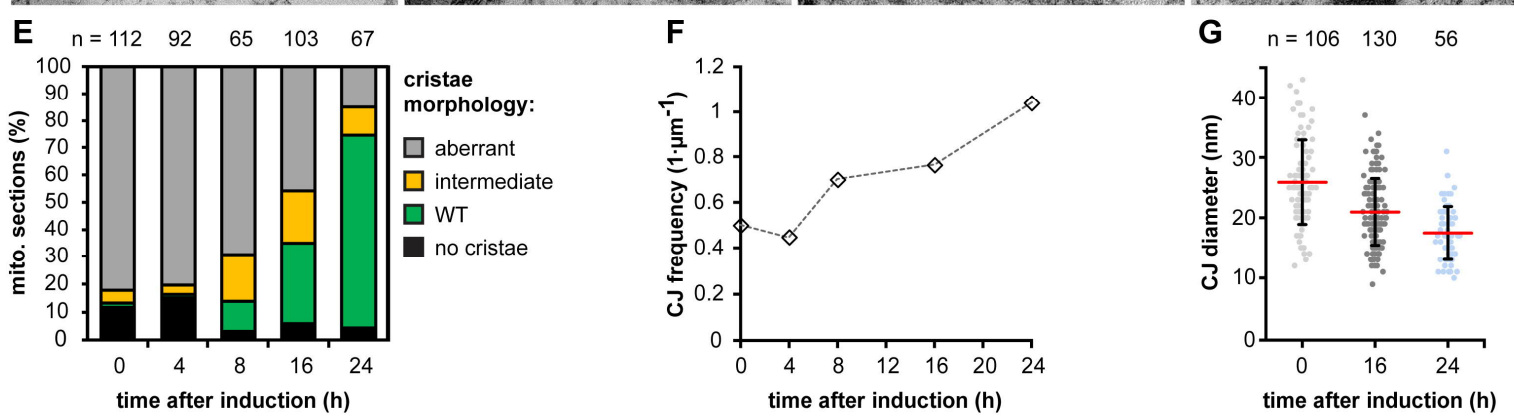

Fig 6. The Mic10-subcomplex is essential for coordination of lamellar cristae formation. (A,B) Live-cell nanoscopy of Mic10-TO cells. Cells expressing COX8A-SNAP were labeled with SNAP-cell SiR and recorded with STED nanoscopy. (A) Cristae architecture in noninduced cells. (B) Cristae architecture after Mic10 re-expression (24 $\mathrm{h}$ induction with doxycycline). STED image data were deconvolved. (C) STED nanoscopy of fixed Mic10-TO cells. Cells were induced for $24 \mathrm{~h}$ and immunolabeled for Mic60 and Mic10-FLAG. Upper row: Cell with weak Mic10 expression. Lower row: Cell with strong Mic10 expression. (D-G) TEM of Mic10-TO cells. (D) Cells induced for the indicated period of time were recorded with TEM. Arrow indicates an intermediately shaped crista. (E) Quantification of the cristae morphology. n: Number of mitochondrial sections. (F) CJ frequency in Mic10-TO cells. Number of CJs was normalized to the length of the OM. The same sections were analyzed as in $(E)$. (G) Diameter of CJs estimated on TEM recordings. n: Number of CJs. Error bars: SD. Scale bars: $10 \mu \mathrm{m}$ (A, B, confocal/conf), $1 \mu \mathrm{m}$ (A, B, C), 0.5 $\mu \mathrm{m}(\mathrm{D})$. 
A
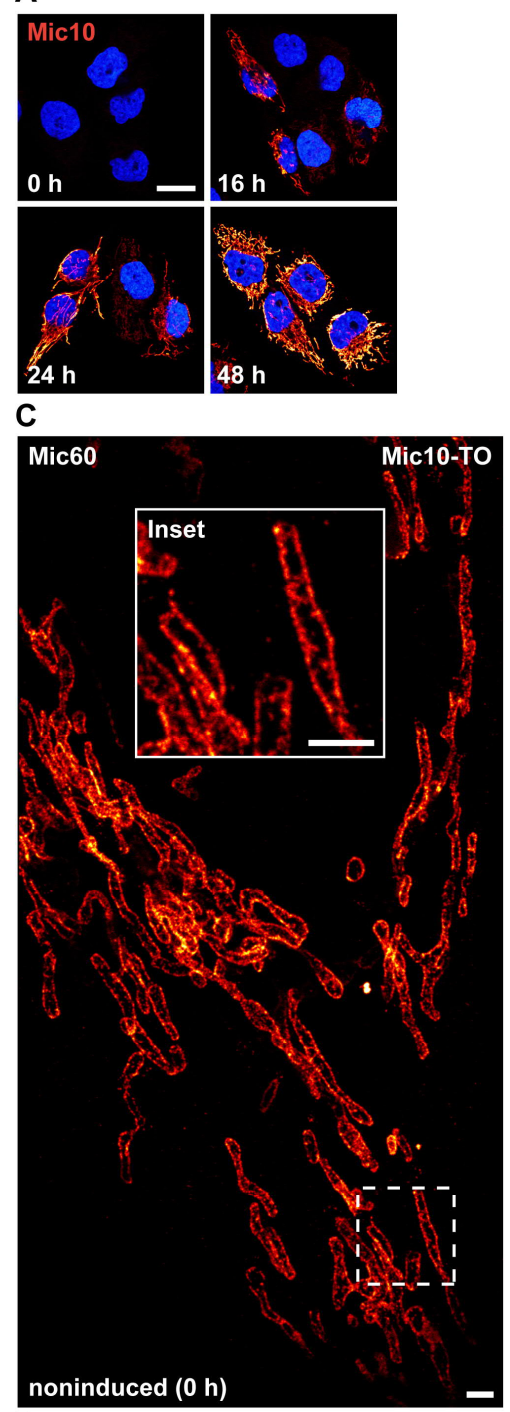

D

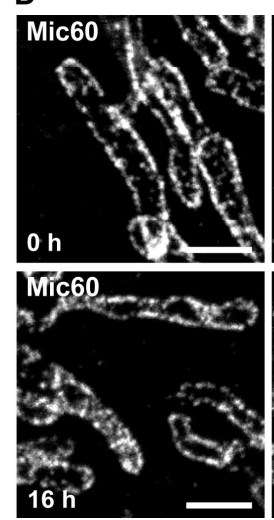

B
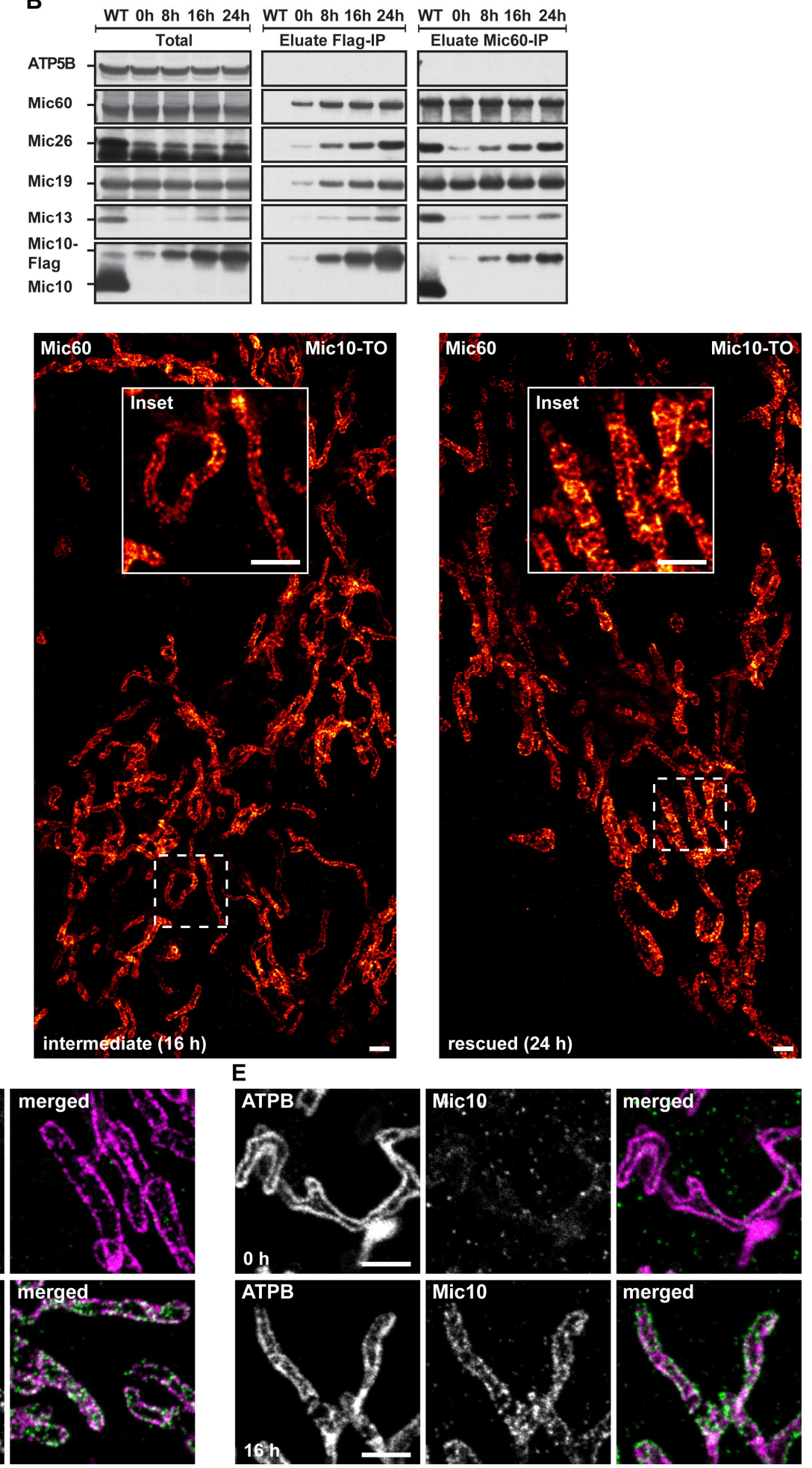

$\mathbf{E}$

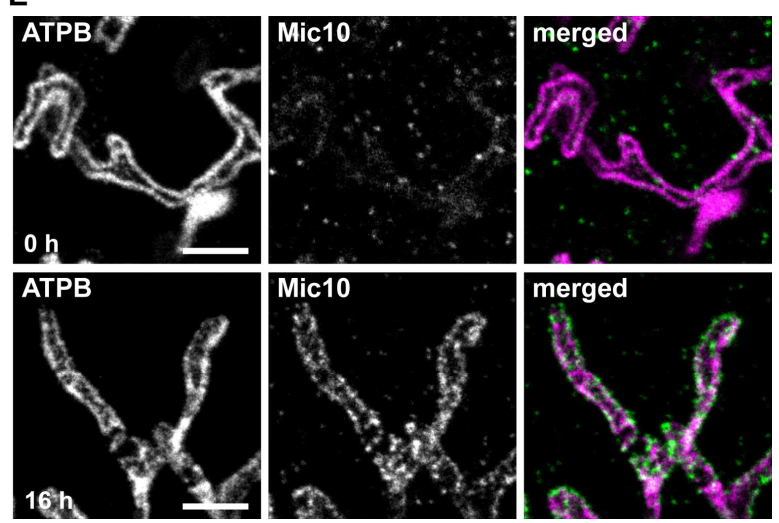

Fig EV3. The Mic10-subcomplex is essential for coordination of lamellar cristae formation. (A-D) Expression of Mic10-FLAG upon induction of Mic10- TO cells. (A) Cells were induced with doxycycline, immunolabeled for Mic10-FLAG, stained with DAPI and subsequently recorded by confocal fluorescence microscopy. (B) Composition of MICOS in induced Mic10-TO cells. FLAG antibodies were used to pull-down Mic10-FLAG and interacting proteins from cell lysates. Mic60-antibodies were used to pull-down Mic60 and interacting proteins from cell lysates. Samples were analyzed by Western blotting. (C) Nanoscopy of Mic10-TO cells. Cells were immunolabeled for Mic60. Images show 2D STED recordings of cells induced for the indicated time period. A dual-color cut-out of the same image (24 h) is shown in Fig 6C. (D, E) Dual-color STED nanoscopy of Mic10-TO cells. Cells were induced for the indicated time points and immunolabeled for Mic60 and Mic10-FLAG or ATPB and Mic10-FLAG, respectively. A larger singlecolor overview including the same image for $0 \mathrm{~h}$ induction is shown in (C). Scale bars: $20 \mu \mathrm{m}(\mathrm{A}), 2 \mu \mathrm{m}(\mathrm{C}), 1 \mu \mathrm{m}$ $(\mathrm{D}, \mathrm{E})$. 
These observations suggest a continuous remodeling of the aberrant tube-like cristae into lamellar cristae controlled by the assembly of the holo-MICOS complex. To test the idea of a continuous remodeling of the mitochondrial architecture, we next analyzed the distribution of Mic10, Mic60 and ATPB in Mic10-TO cells induced for $16 \mathrm{~h}$ using nanoscopy (Fig EV3C-E). In line with the EM data, we observed intermediate phenotypes for the distribution of Mic10, Mic60 and ATPB, further supporting extensive IM remodeling. To investigate the reshaping of the cristae upon expression of Mic10-FLAG in 3D, we performed FIB-SEM that allowed us to visualize whole mitochondria in $3 \mathrm{D}$, but without the resolution to discern individual CJs (Fig

7A, Appendix Fig S6).

A

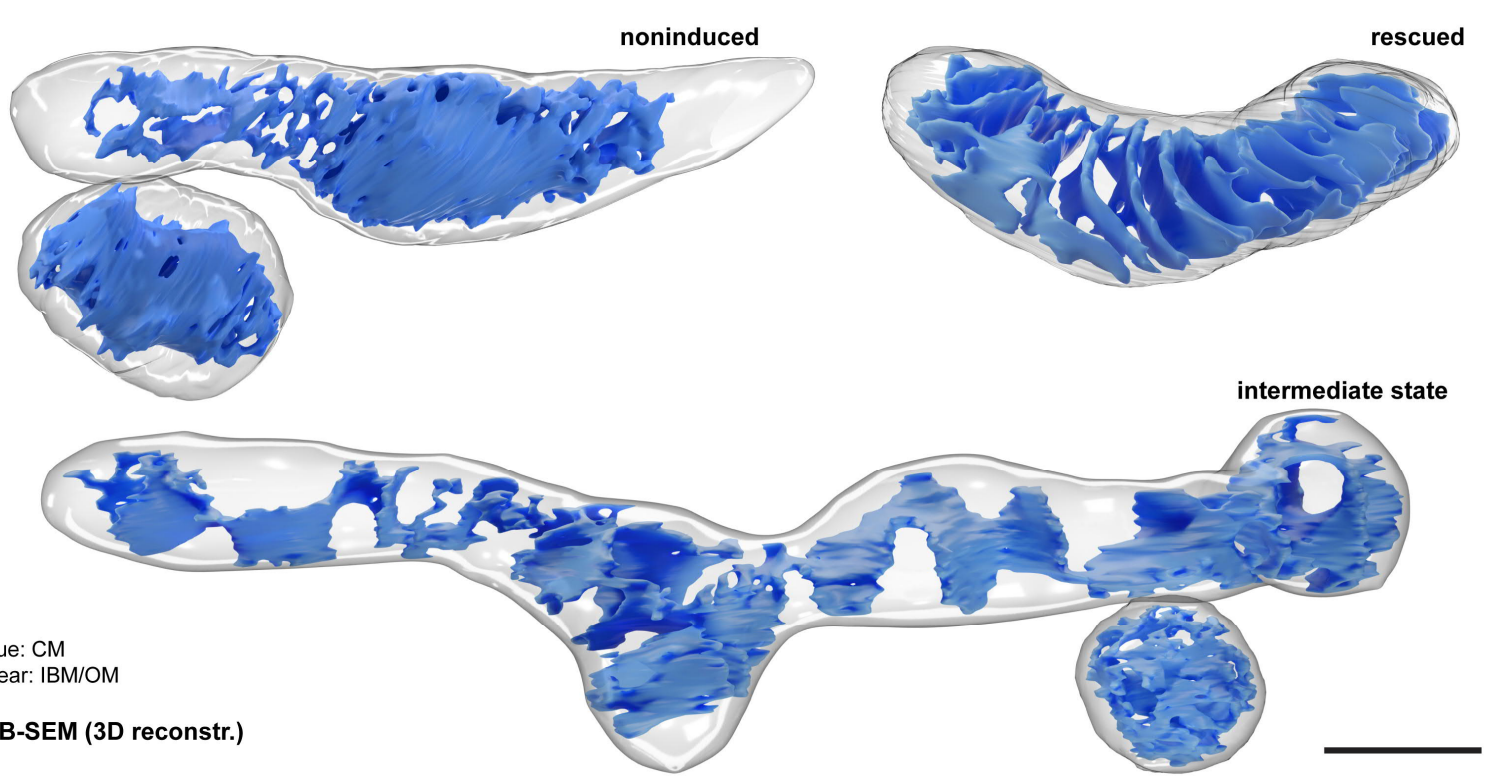

B
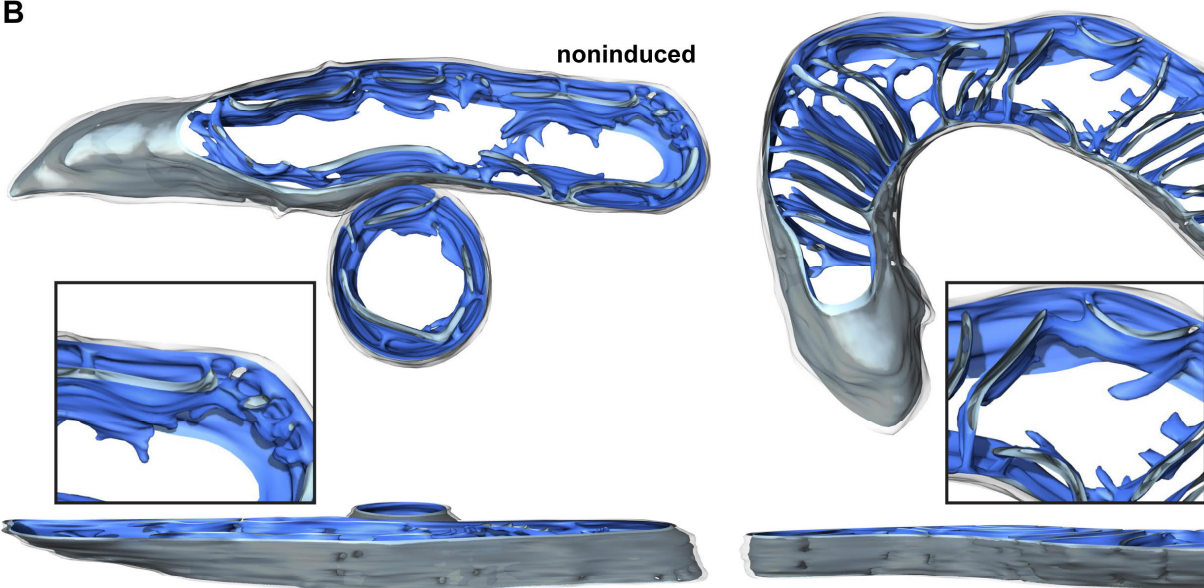

Dark blue: CM/IBM (facing matrix)

Light blue: CM/IBM (facing IMS)

Clear grey: OM

ET (3D reconstr.)

Fig 7. holo-MICOS complex assembly induces remodeling of the mitochondrial inner membrane. (A) FIBSEM of Mic10-TO cells. Cells were induced for 0,16 and $24 \mathrm{~h}$. The representative reconstructions show mitochondria from noninduced, rescued and intermediate cells, as indicated. The OM is shown together with the IBM in clear grey, the CM in blue. (B) ET of Mic10-TO cells. Mic10 expression was induced for $0 \mathrm{~h}$ and $16 \mathrm{~h}$. Shown are reconstructions of representative noninduced and almost completely rescued mitochondria. The OM is displayed in clear grey, the side of the IM that faces the matrix is shown in dark blue. The IM side that faces the IMS is shown in light blue. Scale bars: $500 \mathrm{~nm}$. 
The FIB-SEM data verified the existence of intermediate cristae which are presumably developing from a tube-like structure into a lamellar shape (Fig 7A; Movies EV10-12). Representative reconstructions based on FIB-SEM data display cristae morphologies of a noninduced $(0 \mathrm{~h})$, an intermediate $(16 \mathrm{~h})$ and a largely rescued mitochondrion $(24 \mathrm{~h})$ from Mic10-TO cells (Fig 7A, Movies EV10-12). The 3D structure of the intermediate mitochondrion is suggestive of an arching of the large unfolded CMs (Fig 7A, lower panel, Movie EV12). We propose that these wavy CMs are subsequently converted into individual lamellar cristae.

Next, we analyzed Mic10-TO cells re-expressing Mic10 using ET. In contrast to FIB-SEM, ET recordings allowed us to analyze CJs, although the analyzed volume does nor encompass an entire mitochondrial tubule. The representative reconstructions based on ET data demonstrate that the cristae in mitochondria from noninduced Mic10-TO cells and from rescued Mic10-TO cells are similar to the ones from Mic10-KO and the WT cells, respectively (Fig 7B, Movie EV13 and 14). Like Mic10-KO cells, noninduced cells showed irregularly distributed CJs that connected the tube-like cristae with the IBM (Fig 7B, Movie EV13). In induced cells, we observed mitochondria exhibiting separated lamellar cristae, representing the final stage of recovery, contiguous with interconnected, ragged cristae structures, corresponding to an intermediate stage (Fig 7B, Movie EV14). At lamellar cristae, the CJs were again arranged in line patterns perpendicular to the length axis of the mitochondrion.

Taken together, we conclude that upon re-expression of Mic10-FLAG in Mic10-TO cells, the Mic10-subcomplex is stabilized and interacts with the Mic60-subcomplex. The formation of the holo-MICOS complex is accompanied by a redistribution of MICOS around the mitochondria and a recovery of the lamellar cristae. Our data suggest that during this process the large tube-like cristae of the noninduced Mic10-TO mitochondria furrow, fragment and are thereby converted into separated lamellar cristae. 


\section{Outer membrane fission or fusion is not essential for lamellar cristae formation}

In yeast, the generation of lamellar cristae depends on the mitochondrial fusion machinery (Harner et al., 2016, Kojima, Kakimoto et al., 2019). Because mitochondrial fission and fusion are balanced processes, fission defective mitochondria also exhibit less fusion events. Indeed, mitochondria of $\Delta d n m 1$ yeast cells, which have strongly reduced mitochondrial fission rates, exhibit a substantially reduced number of lamellar cristae, but a high number of branched, tubular cristae (Harner et al., 2016). In order to test if also in higher eukaryotes the formation of lamellar cristae is dependent on mitochondrial fission, we depleted WT cells of DRP1 (also known as DNM1L, dynamin-1-like protein), which is essential for the fission of mitochondrial tubules in higher eukaryotes (Bleazard, McCaffery et al., 1999, Cereghetti, Stangherlin et al., 2008, Lee, Jeong et al., 2004).

Corroborating previous reports (Ban-Ishihara, Ishihara et al., 2013), WT cells depleted of DRP1 by RNAi for 7 days exhibited hyperfused mitochondrial networks and numerous lamellar cristae (Fig EV4A and B). This finding suggests that in HeLa cells fission of mitochondrial tubules is not essential for the generation of lamellar cristae.

In mammalian cells, the fusion of the mitochondrial OM is regulated by the two mitofusins MFN1 and MFN2, two highly conserved dynamin-related GTPases, which exhibit distinguishable functions (Giacomello, Pyakurel et al., 2020, Ishihara, Eura et al., 2004). To investigate whether OM fusion is essential for lamellar crista formation, we depleted HeLa cells of MFN1 or MFN 2 or MFN1 together with MFN2. Depletion of these proteins resulted in a mild cristae phenotype, but lamellar cristae were still observed (Fig EV4C-D).

We conclude that in mammalian cells OM fission or fusion are not essential for the development of lamellar cristae. 
A

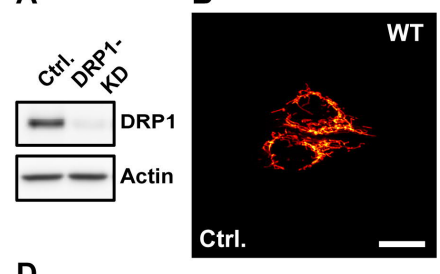

D

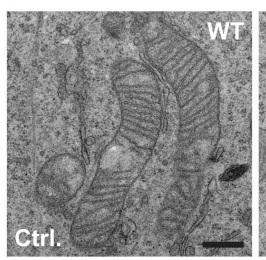

$\mathbf{F}$

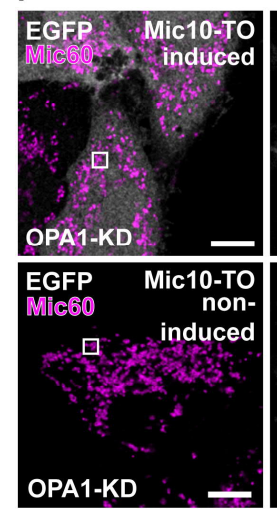

H

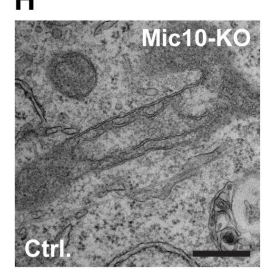

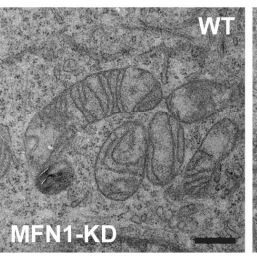

MFN1-KD
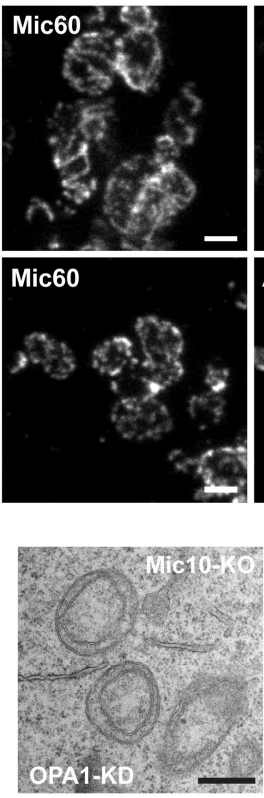
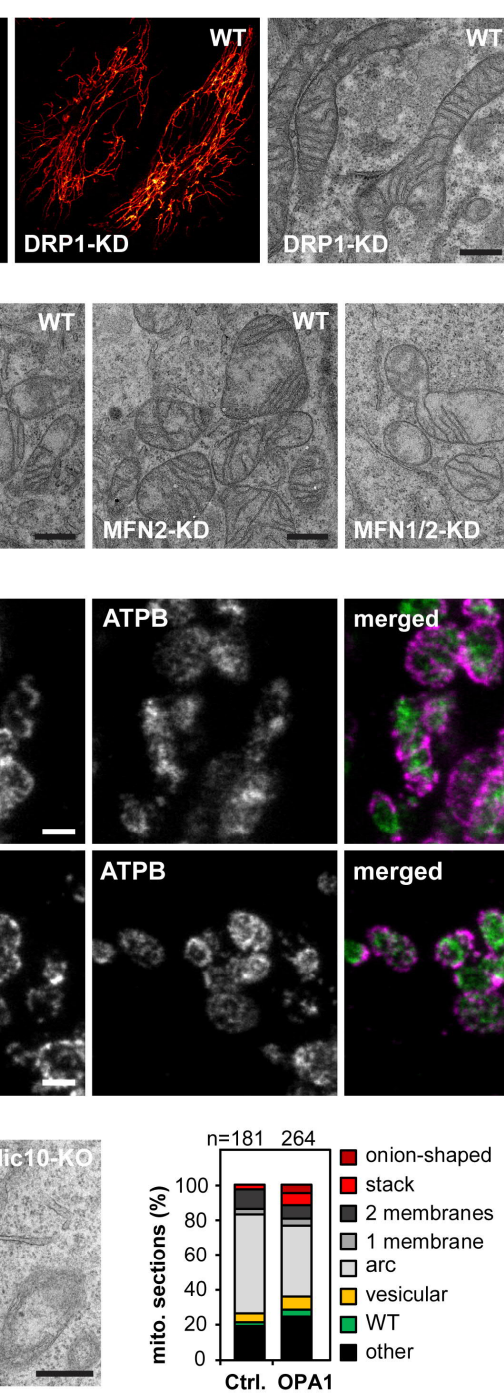

C
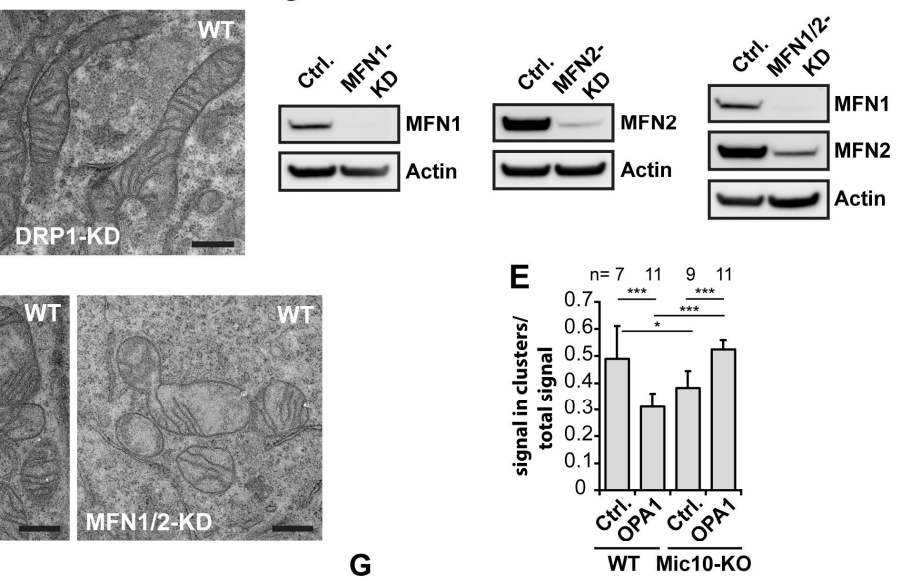

G
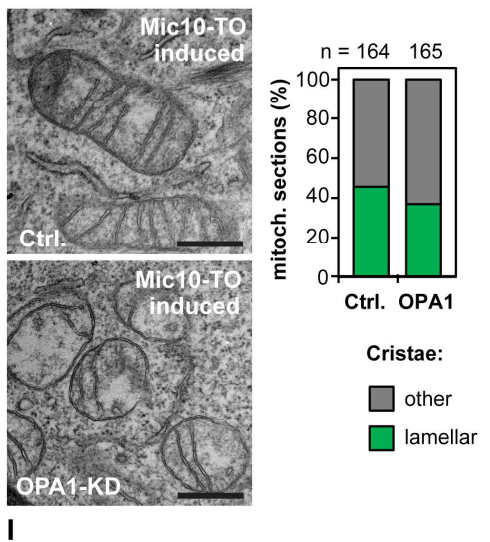

I

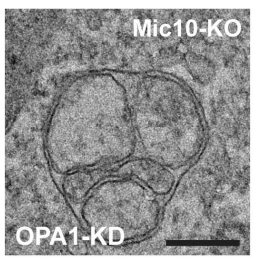

Fig EV4. Formation of lamellar cristae does not require mitochondrial tubule fusion or fission; Mic10 influences the distribution of Mic60. (A-B) Knockdown (KD) of DRP1 in HeLa cells. WT cells were transfected with a scrambled control (Ctrl.) or a siRNA plasmid against DRP1 for 7 days. (A) Cells were analyzed by Western blotting. (B) Ctrl. and DRP1-KD cells were immunolabeled for TOM20 and analyzed by confocal fluorescence microscopy (left) or analyzed by TEM (right). (C, D) KD of MFN1 and MFN2 in HeLa cells. WT cells were transfected with Ctrl. or siRNA pools against MFN1, MFN2 or both proteins together for 4 days. (C) Cells were analyzed by Western blotting. (D) Cells were analyzed by TEM. (E) Mic60-cluster analysis of OPA1-deficient HeLa cells. WT cells and Mic10-KO cells were either transfected with a scrambled control (Ctrl.) or a siRNA pool against OPA1 (OPA1-KD). Cells were immunolabeled against Mic60 and recorded by 2D STED nanoscopy. Images were automatically segmented and the ratio of Mic60 signals in small distinct clusters (Mic60 assemblies $\leq 0.0225 \mu \mathrm{m}^{2}$ ) to the total Mic60 signals was determined. Higher values indicate smaller Mic60 assemblies, lower values the formation of larger Mic60 assemblies. Bars indicate mean. Error bars indicate SD. For statistics a One-way Anova Assay was performed. ${ }^{*}: p \leq 0.05,{ }^{* * *}: p \leq 0.001$. $n$ : Number of cells that were analyzed. $(F-G)$ Mic10 re-expression after OPA1 depletion. Mic10-TO cells were transfected with a scrambled control (Ctrl.) or a siRNA pool against OPA1 (OPA1-KD) for $48 \mathrm{~h}$. Afterwards, Mic10-FLAG expression was induced for $24 \mathrm{~h}$. (F) Cells were immunolabeled for Mic60 and ATPB and recorded by STED nanoscopy. Left panel: Confocal overview images taken from induced cells (cytosolic reporter EGFP, upper panel) and noninduced cells (lower panel). Right: STED images from mitochondria from the areas indicated by rectangles. (G) TEM of Ctrl. and OPA1-deficient Mic10-TO cells after re-expression of Mic10-FLAG. Right: Quantification of the cristae morphology. n: Number of mitochondrial sections analyzed. (H-I) Knockdown of OPA1 in Mic10-KO cells. $(\mathrm{H})$ Left: Cells were transfected with a Ctrl. or a siRNA pool against OPA1 and analyzed by TEM. Right: Recordings were assigned to the indicated crista phenotypes. n: Number or mitochondrial sections analyzed. (I) Recording of a mitochondrion of a Mic10-KO cell depleted for OPA1 exhibiting septa and septa junctions. Scale bars: $20 \mu \mathrm{m}$ (B, light microscopy), $10 \mu \mathrm{m}$ ( $\mathrm{F}$, overview), 500 nm (B, TEM; D; F, inset; G; H), 250 nm (I). 


\section{Deletion of OPA1 induces a moderate cristae phenotype in WT cells}

A substantial body of evidence demonstrates that the dynamin-like GTPase OPA1 (Mgm1 in yeast) influences cristae architecture (Cogliati et al., 2016, Glytsou, Calvo et al., 2016, Kondadi, Anand et al., 2019, MacVicar \& Langer, 2016, Patten, Wong et al., 2014, Ramonet, Perier et al., 2013). To further investigate the role of OPA1 in cristae development, we first efficiently depleted OPA1 by RNAi in WT and Mic60-KO cells (Fig 8). Compared to Mic10-KO and Mic60-KO cells, OPA1 depleted WT cells had a moderate cristae phenotype, as the portion of mitochondrial TEM sections showing cristae lamellae was reduced by only about $10 \%$; still the cristae often appeared shorter, disordered or partly swollen (Fig 8B and C), fully in line with previous studies (Barrera, Koob et al., 2016, Glytsou et al., 2016, Olichon, Baricault et al., 2003).

Yeast $\Delta m g m l$ cells have been reported to contain septa, i.e. IM structures that divide the mitochondrial matrix in two physically separated compartments (Harner et al., 2016, Kojima et al., 2019, Sesaki, Southard et al., 2003).

A

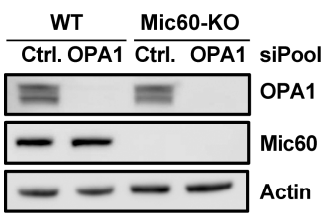

B
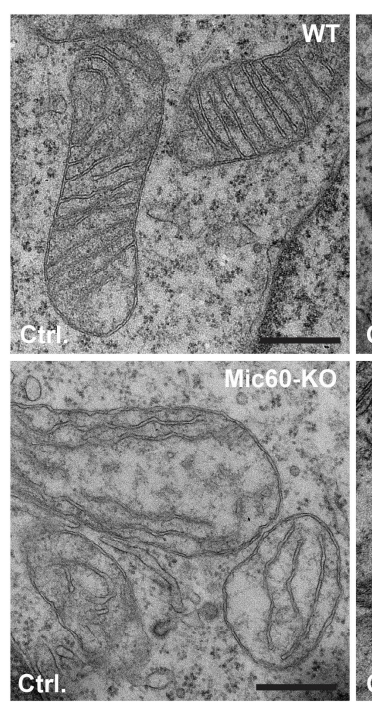

C
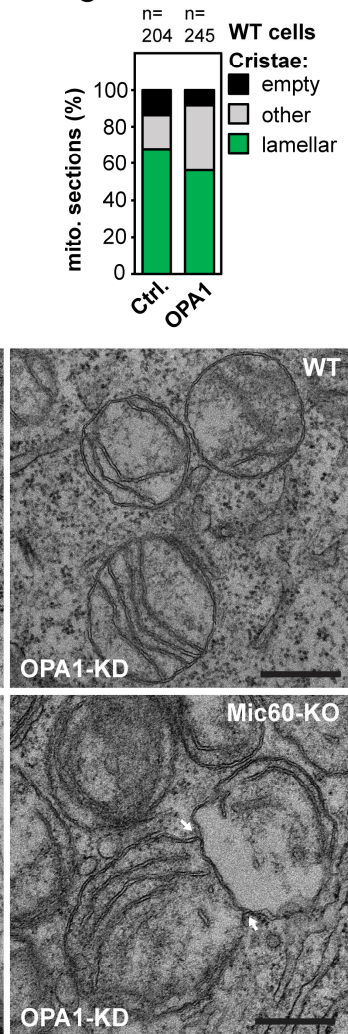

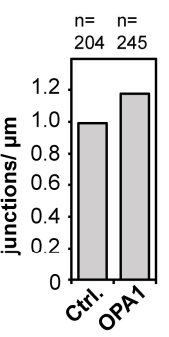

D
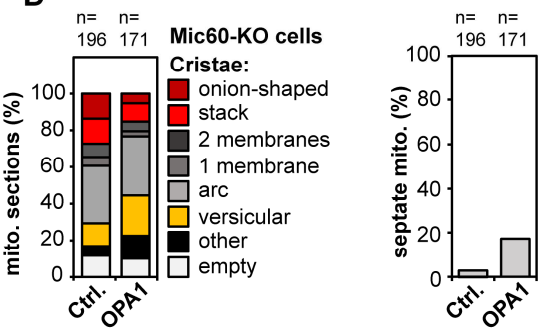

E

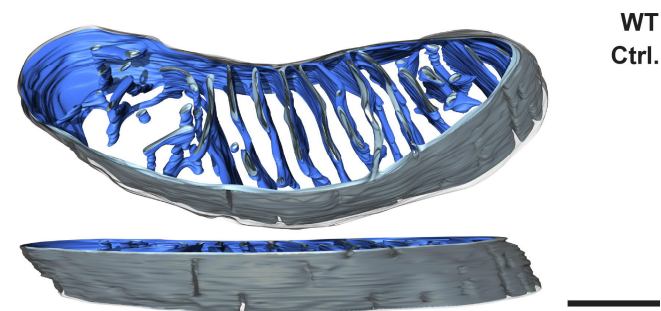

$\mathbf{F}$
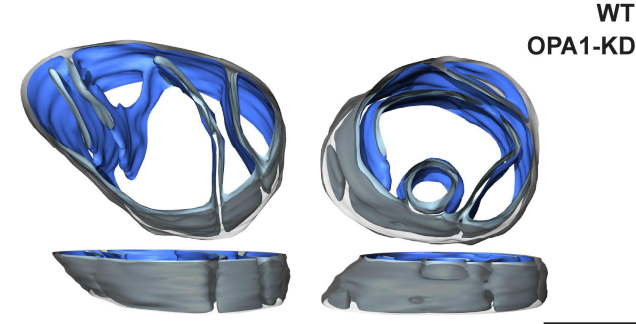

Fig 8. OPA1 influences the inner membrane morphology. (A-F) Knockdown (KD) of OPA1 in HeLa cells. WT and Mic60-KO cells were transfected with a scrambled control (Ctrl.) or siRNA pools against OPA1 (OPA1-KD) for 5 days. (A) Cell lysates were analyzed by Western blotting. (B) TEM recordings of OPA1 depleted WT and Mic60KO cells. Arrows point to a septum. (C,D) Quantification of the cristae morphology of OPA1-deficient WT (C) and Mic60-KO (D) cells. The number of CJs was normalized to the length of the OM (C). The number of septate mitochondria in Mic60-KO cells was estimated (D). (E,F) ET of mitochondria from OPA1-KD cells. WT cells were transfected with a scrambled control $(\mathrm{E})$ or siRNA pools against OPA1 (F) for $48 \mathrm{~h}$. Reconstructions of representative mitochondria are displayed. The $\mathrm{OM}$ is displayed in clear grey, the side of the IM that faces the matrix is shown in dark blue. The IM side that faces the IMS is shown in light blue. Scale bars: $500 \mathrm{~nm}(\mathrm{~B}), 250 \mathrm{~nm}$ $(E, F)$. 
Such septa are the result of incomplete IM fusion after tubule fusion (Harner et al., 2016). In WT HeLa cells a single crista is often connected by CJs to both sides of the mitochondrion. Thus, septa could easily be mistaken by cristae on TEM recordings. However, in Mic60-KO cells, septa junctions should be unequivocally recognized as almost no CJs are formed in the absence of Mic60. Indeed, we observed in $\sim 15 \%$ of the mitochondria from OPA1-deficient Mic60-KO cells such septa (Fig 8B and D). Otherwise, the mitochondria were phenotypically similar to the scrambled RNAi Mic60-KO control (Fig 8B and D). The observation of septa in OPA1 depleted Mic60-KO cells suggested that such septa also exist in OPA1 depleted WT cells. Therefore, a fraction of CJs in these cells (Fig 8C) might actually represent septa junctions. To determine if in WT cells depleted for OPA1 the majority of IM structures are septa or cristae, we performed ET (Fig 8E and F, Movies EV15 and 16). Reconstructions revealed that most IM structures did not continuously cross the entire mitochondrion within the $200 \mathrm{~nm}$ thick sections and therefore represent disordered lamellar cristae, not septa (Fig 8F, Movie EV16). 


\section{OPA1 influences the formation of MICOS assemblies and stabilizes tubular CJs}

OPA1 and Mic10 influence the size and the distribution of Mic60 assemblies

We next investigated the influence of OPA1 on the distribution of Mic60. To this end, we immunolabeled cells for Mic 60 and the ATPB subunit of the $\mathrm{F}_{1} \mathrm{~F}_{0}$-ATP synthase and performed STED nanoscopy (Fig 9A). In OPA1-deficient mitochondria we found conspicuous ring- and rib-like Mic60 assemblies that were significantly larger than the rod shaped Mic60 clusters in the WT control (Fig 9A). Because in Mic19-deficient cells the occurrence of elongated Mic60 assemblies was Mic10-dependent (Fig 4F, Fig EV2F), we next asked if Mic10 also controls the formation of the Mic60 assemblies in OPA1 depleted cells. We found that in Mic10-KO cells devoid of OPA1, Mic60 did not form extended assemblies but localized in small clusters (Fig 9B, Fig EV4E). In these cells, also the distribution of Mic60 in opposite distribution bands observed in Mic10-KO cells had disappeared, as the clusters were scattered across the mitochondria (Fig 9B). To test if in cells depleted of Mic10 and OPA1 the distribution of Mic60 into large assemblies can be induced by re-expression of Mic10, we depleted Mic10-TO cells for OPA1 for 48h and subsequently induced Mic10 expression for $24 \mathrm{~h}$ (Fig EV4F). STED images showed that Mic10-FLAG expression resulted in the redistribution of Mic60 clusters into larger Mic60 assemblies (Fig EV4F). In addition, TEM demonstrated that OPA1 depleted Mic10-TO cells, expressing Mic10-FLAG for 24 hours, had developed disordered cristae lamellae, comparable to OPA1 depleted WT cells (Fig EV4G). This demonstrates that Mic10 induces cristae remodeling also in OPA1-deficient cells. We conclude that OPA1 is not essential for formation of cristae lamellae but controls, together with Mic 10, the formation and distribution of Mic60 assemblies.

\section{OPA1 stabilizes tubular CJs in Mic10-KO cells}

Since the simultaneous depletion of both Mic10 and OPA1 led to randomly scattered Mic60 clusters, we next investigated if the mitochondria in these cells still featured CJs. TEM of OPA1 depleted Mic10-KO cells revealed, except for a strong fragmentation of the mitochondrial tubules, as similar cristae phenotype as seen in Mic10-KO cells (Fig EV4H). However, we observed a reduction of CJs in Mic10-KO cells devoid of OPA1 (Fig 9C). In Mic10-KO cells transfected with scrambled siRNAs about $60 \%$ of the TEM sections of mitochondria showed at least one $\mathrm{CJ}$, whereas after depletion of OPA1 only $36 \%$ of the mitochondria showed at least one CJ (Fig 9C). About $50 \%$ (about $15 \%$ in controls) of these junctions were associated with septa and thus are likely to represent septa junctions (Fig $9 \mathrm{C}$ and Fig EV4I). Hence, in non-septate mitochondria devoid of Mic10 and OPA1 the number of CJs was strongly decreased by about $66 \%$. This suggests an important role of OPA1 for the stability of tubular CJs in the absence of Mic 10 .

Taken together, OPA1 is required for proper cristae architecture, although its depletion results only in a mild phenotype compared to Mic10 or Mic60 knockouts. A simultaneous depletion of both OPA1 and Mic10 further reduced the number of CJs suggesting that OPA1 works in 
concert with the Mic10-subcomplex to stabilize tubular CJs. Additionally, OPA1 and Mic10 antagonistically determine the distribution and size of Mic60 assemblies.

A

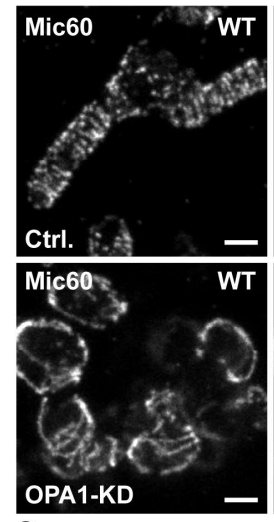

C

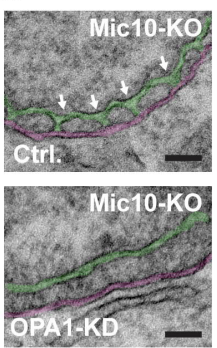

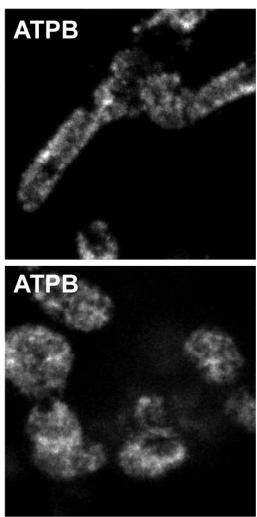

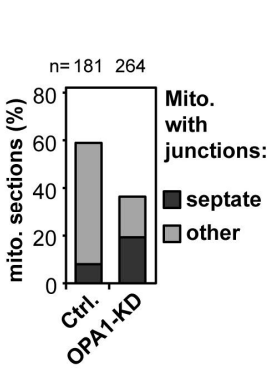

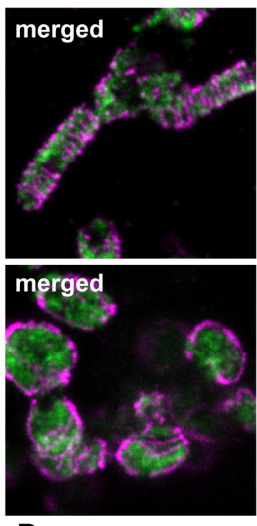

D

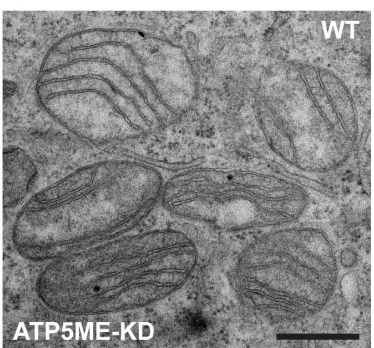

B
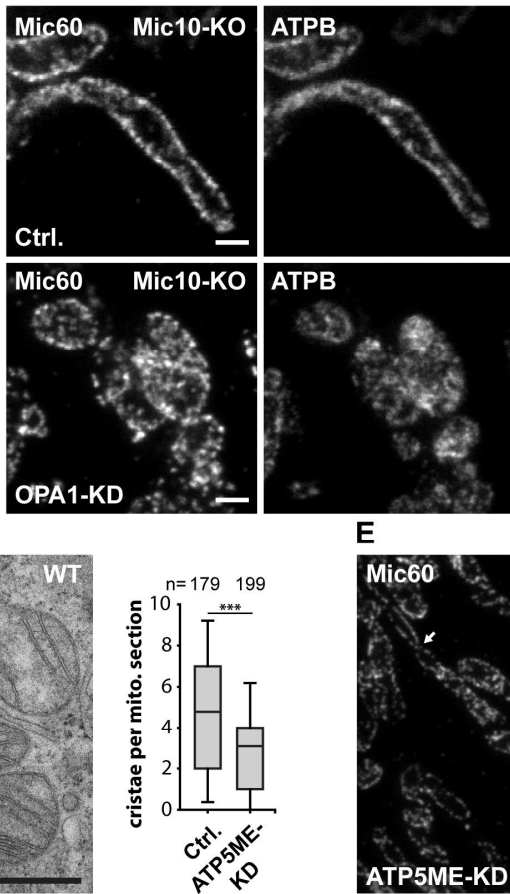

E
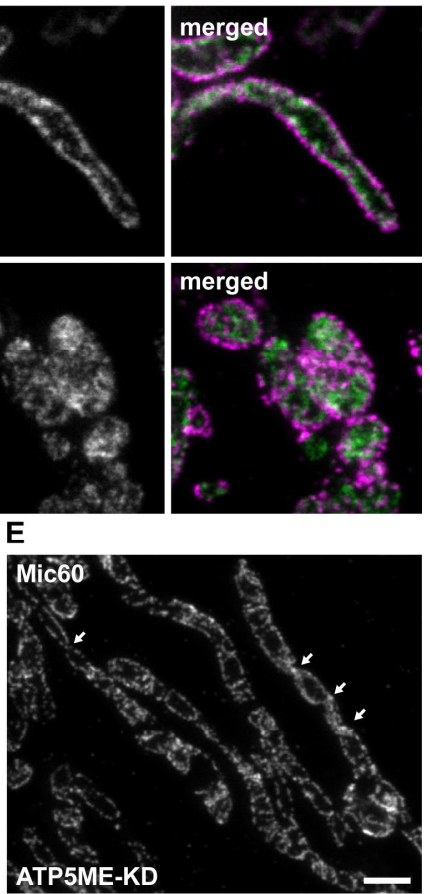

Fig 9. OPA1 affects the Mic60 distribution and stabilizes tubular CJs. (A-C) WT and Mic10-KO cells were transfected with a scrambled control (Ctrl.) or siRNA pools against OPA1 (OPA1-KD) for 72 h. (A,B) STED nanoscopy of OPA1 depleted WT (A) and Mic10-KO (B) cells. Cells were immunolabeled for Mic60 and ATPB. (C) TEM of OPA1 depleted Mic10-KO cells. Left: Representative TEM recordings showing CJs marked by arrows in a control cell and a similar view for an OPA1-KD cell. The CMs are colored in green and the OM and IBM together in magenta. Right: Quantification of mitochondria containing CJs or septa junctions. Sections that contained CJ-like structures were analyzed. Of these, the number of sections exhibiting septa or cristae structures (other) was quantified. (D, E) Depletion of ATP5ME in HeLa cells. Cells were transfected with a scrambled control or siRNA pools against ATP5ME for 4 days. (D) TEM recording (left) and quantification of the number of cristae per mitochondrial section (right). (E) Cells depleted for ATP5ME were immunolabeled for Mic60 and recorded with STED. The arrows point to crossing points of the opposite Mic60 distribution bands. $n$ : Number of mitochondrial sections analyzed. Boxes indicate $25^{\text {th }}$ and $75^{\text {th }}$ percentile. Lines indicate median. Bars indicate mean \pm SD. MannWhitney test was used to compare samples. ${ }^{* \star \star}: p \leq 0.001$. Scale bars: $500 \mathrm{~nm}(A, B, D), 100 \mathrm{~nm}(C), 1 \mu \mathrm{m}(E)$. 


\section{The dimeric $F_{1} F_{0}-A T P$ synthase affects the cristae architecture and the Mic60 distribution}

Next to MICOS and OPA1, the mitochondrial $\mathrm{F}_{1} \mathrm{~F}_{0}$-ATP synthases is a key player for shaping the IM (Kuhlbrandt, 2019). Mitochondrial $\mathrm{F}_{1} \mathrm{~F}_{\mathrm{o}}$-ATP synthases generally form dimers, which can assemble into long ribbons that contribute to the shape of the cristae (Blum et al., 2019, Dudkina, Sunderhaus et al., 2006, Strauss et al., 2008). The $\mathrm{F}_{1} \mathrm{~F}_{\mathrm{o}}$-ATP synthase subunit ATP5ME (also known as ATP synthase subunit $e$, ATP5K or ATP5I) is involved in $\mathrm{F}_{1} \mathrm{~F}_{0}$-ATP synthase dimerization (Arnold, Pfeiffer et al., 1998, Habersetzer, Larrieu et al., 2013, QuintanaCabrera et al., 2018). To disturb dimer formation we used RNAi to deplete ATP5ME in HeLa cells (Fig EV5A). Cells devoid of ATP5ME contained mostly large spherical mitochondria, but also some elongated tubular mitochondria (Fig 9D, Fig EV5A and B). Compared to the depletion of MICOS or of the Mic10-subcomplex, the IM generally exhibited a mild phenotype. As reported previously, the mitochondria of cells depleted for ATP5ME contained fewer, often slightly disordered lamellar cristae (Fig 9D, Fig EV5B) (Arnold et al., 1998, Habersetzer et al., 2013, Paumard, Vaillier et al., 2002, Quintana-Cabrera et al., 2018, Rabl et al., 2009). As the $\mathrm{F}_{1} \mathrm{~F}_{\mathrm{o}}$-ATP synthase has been shown to interact with the MICOS complex in yeast (Eydt et al., 2017, Rampelt et al., 2017a, Rampelt \& van der Laan, 2017), we next investigated the distribution of Mic60 in cells depleted for ATP5ME.
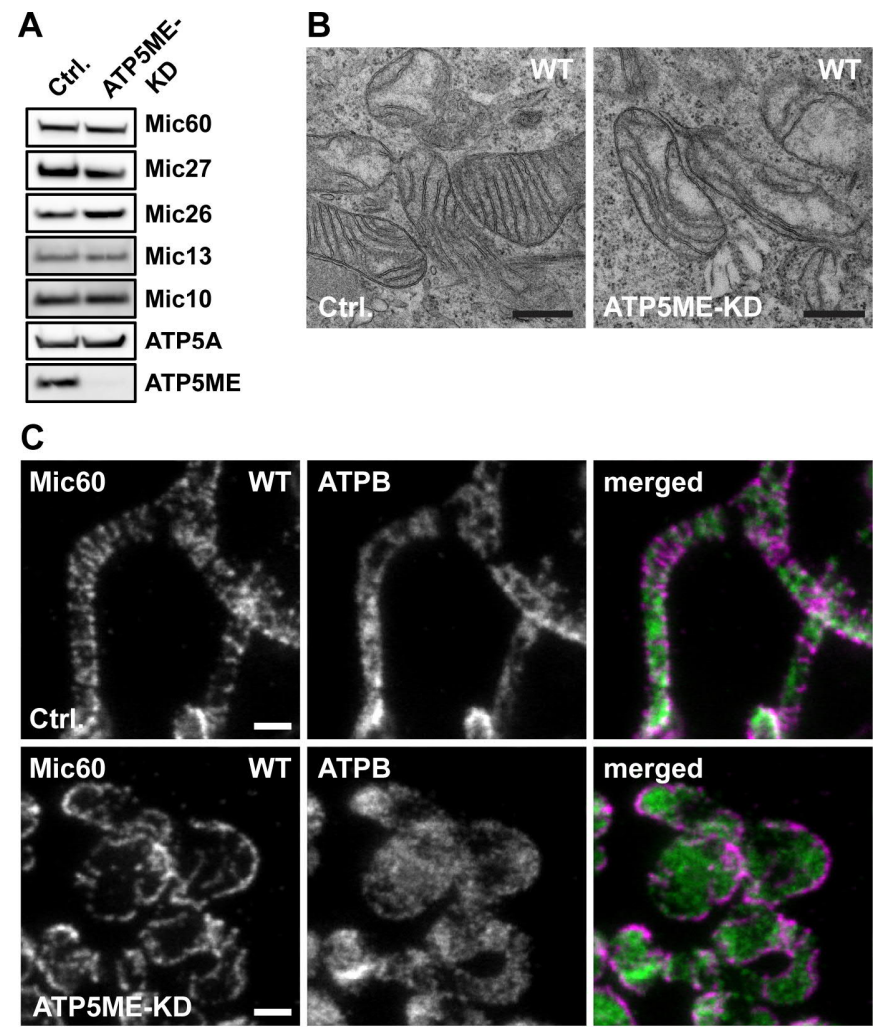

Fig EV5. Dimers of the $F_{1} F_{0}$-ATP-Synthase influence the MICOS distribution. (A-C) Knockdown of ATP5ME in HeLa cells. Cells were transfected with a Ctrl. or a siRNA pool against ATP5ME (ATP5ME-KD) and analyzed after 4 days. (A) Protein levels of ATP5ME, ATP5A and MICOS proteins in cell lysates as analyzed by Western blotting. (B) TEM recordings of ATP5ME depleted cells. (C) STED nanoscopy of ATP5ME depleted cells. Cells were immunolabeled for Mic60 and ATPB. Scale bars: $500 \mathrm{~nm}$. 
In the spherical, more aberrant mitochondria, Mic60 seemed to be arranged in long stripes encircling the organelles (Fig EV5B). In the tubular mitochondria, Mic60 primarily formed clusters that were localized on opposite sides of the mitochondrial tubules (Fig 9E). Between these bands, we occasionally observed a stripe-like arrangement of the Mic60 clusters perpendicular to longitudinal axis of the mitochondria (Fig 9E). This overall distribution of Mic60 in ATP5ME depleted cells (Fig 9E) was reminiscent of the Mic60 distribution in Mic10KO cells (Fig 4A), or in cell types that exhibit fewer or smaller cristae (Stoldt et al., 2019). Similar to the situation in these cell types, the narrow Mic60 bands were also often twisted, resulting in a helical arrangement of the Mic60 clusters (Fig 9E). The ATP5ME-dependent relocalization of MICOS suggests that the dimeric $\mathrm{F}_{1} \mathrm{~F}_{\mathrm{o}}$-ATP synthases support an even distribution of MICOS, and consequently of the CJs, around the mitochondrial tubules. Indeed, when using Mic10-FLAG as a bait in co-IP experiments, we co-isolated also ATP5B suggesting a physical interaction of the $\mathrm{F}_{1} \mathrm{~F}_{\mathrm{o}}$-ATP synthase with the Mic10-subcomplex in human cells (Appendix Fig S5A).

Taken all together, we conclude that in human cells, the two MICOS-subcomplexes have different functions. The Mic60-subcomplex, which is stable in the absence of the Mic10subcomplex, is essential for the maintenance of CJs and the stability of the holo-MICOS complex. The Mic10-subcomplex is essential for lamellar cristae formation. Formation of the holo-MICOS complex mediates extensive remodeling of pre-existing unstructured cristae into individual lamellae and also the formation of secondary CJs. We found that both OPA1 and Mic10 differently influence the distribution and size of MICOS assemblies. Finally, our data show that dimers of the $\mathrm{F}_{1} \mathrm{~F}_{\mathrm{o}}$-ATP synthase influence the cristae shape as well as the distribution of the MICOS complex, together demonstrating multiple functional interactions of the membrane shaping proteins involved in cristae formation. Thereby, as detailed below, our findings support a new model for the formation of cristae in higher eukaryotes. 


\section{Discussion}

In this work, we investigated the interplay of the major determinants of cristae formation in higher eukaryotes, namely the Mic10- and the Mic60-subcomplexes of MICOS, OPA1 and the $\mathrm{F}_{1} \mathrm{~F}_{\mathrm{o}}$-ATP synthase. Our data suggest significant differences in cristae formation of higher and lower eukaryotes. We demonstrate that cristae development in human mitochondria is largely independent from mitochondrial fusion-fission dynamics whereas fusion of mitochondria is essential for lamellar cristae formation in the yeast S. cerevisiae (Harner et al., 2016).

Another significant difference exists in the stability of the Mic10-subcomplex that depends on the Mic60-subcomplex in human cells, whereas it assembles in a Mic60-independent manner in yeast (Anand et al., 2016, Bohnert et al., 2015, Friedman et al., 2015, Guarani et al., 2015, von der Malsburg et al., 2011).

This study shows that upon re-formation of MICOS, the aberrant IM structures of Mic10-KO and Mic60-KO cells are converted into WT cristae, rather than being replaced by new normally shaped CMs. The observed repair mechanisms allow to draw conclusions on the mechanisms that are involved in de novo cristae biogenesis (Fig 10A). Furthermore, the fact that human Mic10-KO cells still form CJs, but exhibit an aberrant cristae architecture, allowed us to disentangle CJ formation from lamellar cristae formation and to investigate the distinct functions of the two MICOS subcomplexes.

\section{Opposite distribution bands}

Our STED and 3D MINFLUX data show that in mitochondria of Mic10-KO cells, the Mic60 clusters are distributed along two narrow opposite distribution bands. As our FIB-SEM, ET, and 3D SIM data consistently show that in the absence of the Mic10-subcomplex the cristae are large rotationally symmetric tube-like structures that are oriented parallel to the OM, the distribution of Mic60 in opposite distribution bands is presumably not a consequence of the cristae morphology. In fact, such Mic60-distribution bands, which can adopt different width, have been previously reported in several WT cell types (Jans et al., 2013, Stoldt et al., 2019). However, this enrichment of Mic60 clusters on opposite sides of the mitochondrial tubules was almost invisible in the wild type cells we used in this study. Different to the situation in e.g. yeast cells or primary fibroblasts, these wild type HeLa cells exhibit large well-developed lamellar cristae, each featuring several adjacent CJs, which almost encircle a mitochondrion. Therefore, in these cells the visibility of the Mic60 distribution bands, which reflect the CJ distribution, might be concealed because they are much wider or highly twisted. We show in this study that the re-distribution of Mic60 in such opposite distribution bands can be induced by several means, including the depletion of ATP5ME, of Mic13, or of Mic10. The prevalence of this phenomenon suggests that these opposite distribution bands are an important structure providing element of mitochondria (Jans et al., 2013, Stoldt et al., 2019). 


\section{Distribution of CJs}

This study also demonstrates that the Mic60-subcomplex is necessary for the generation of CJs, whereas the Mic10-subcomplex is required for lamellar cristae formation. The remodeling of the IM and the generation of secondary CJs during re-expression of Mic10 in Mic10-TO cells are accompanied by a redistribution of MICOS and of the CJs (Fig 10B, Fig 6C, Fig EV3C-E). Next to the Mic10-subcomplex, the dimeric $\mathrm{F}_{1} \mathrm{~F}_{\mathrm{o}}$-ATP synthase, as well as OPA1 influence the distribution of the Mic60-complex. Specifically, in the absence of OPA1, Mic10 induces the formation of extended Mic60 structures, suggesting that OPA1 restricts the size of holo-MICOS assemblies. These findings point to antagonistic functions of OPA1 and Mic10 in the regulation of the distribution and size of the Mic60-subcomplexes (Fig 10C and D).

\section{Recovery of the Mic10 phenotype provides hints for cristae biogenesis}

Remarkably, in yeast the detached IM structures of MICOS-deficient cells feature a similar protein composition as CMs in WT cells (Harner et al., 2016, Harner, Unger et al., 2014), suggesting that not CM formation, but the shaping of the IM is defective in MICOS-deficient cells. Upon re-expression of Mic10 in Mic10-TO cells, unstructured large CMs forms an undulating pattern and develop into multiple lamellar cristae (Fig 10A, Fig 7A, lower panel). Concurrently, MICOS assembly triggers a redistribution of the CJs and the formation of new, secondary CJs, as also observed in Mic10-TO and Mic60-TO cells (Fig 10A and B, Fig 6C, Fig 5F and G). Therefore, the assembly of the holo-MICOS complex can be regarded as a switch controlling the efficient conversion of unstructured cristae into lamellar cristae (Fig 10A). We postulate that the unstructured cristae found in Mic10-KO cells represent a trapped intermediate structure that also occurs during normal cristae formation. We propose that in wild type mitochondria these intermediates are smaller and short lived and are rapidly re-shaped into lamellar cristae following the same principle as observed in rescued Mic10-TO cells (Fig 10A and B, Fig 7A, Fig 6D).

\section{A new model of cristae biogenesis}

Our findings suggest that lamellar cristae biogenesis in higher eukaryotes starts in a MICOS independent way by an unstructured infolding of the IM, followed by MICOS-controlled restructuring of this infolding, including secondary CJ formation (Fig 10A). The positioning and shape of the CJs is fine-tuned by an interplay between the Mic10-subcomplex, OPA1 and the $\mathrm{F}_{1} \mathrm{~F}_{\mathrm{o}}$-ATP synthase. As lamellar cristae usually occur in densely stacked groups (Stephan et al., 2019) it is tempting to assume that the cristae of one group originate from a single precursor CM (Fig 10A). In mitochondria with a different cristae architecture, this cristae biogenesis pathway might also prove to be prevalent, as the reshaping of a larger cristae precursor into several individual cristae could principally lead to any cristae shape. 
A

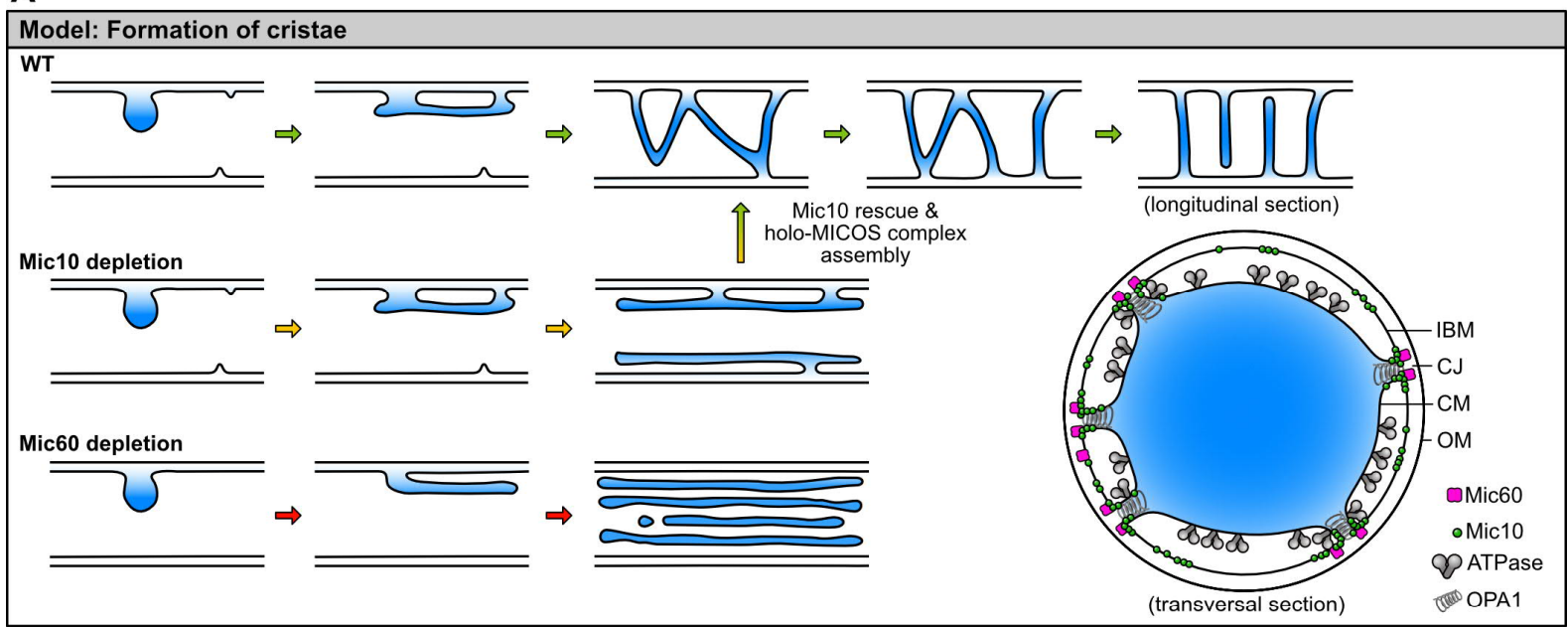

B

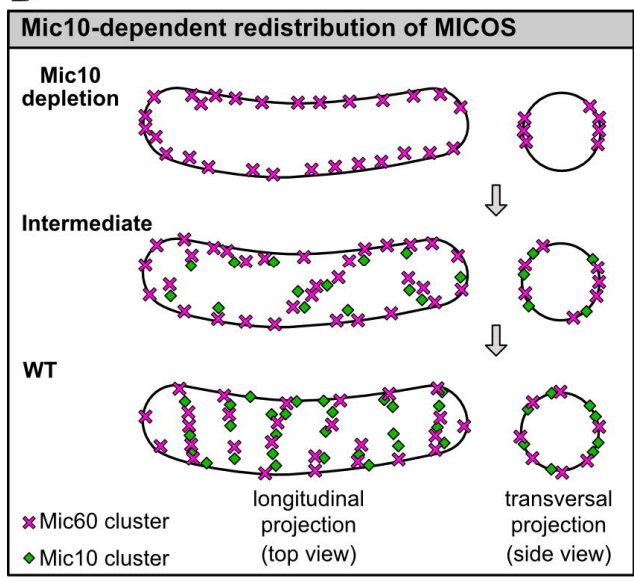

C

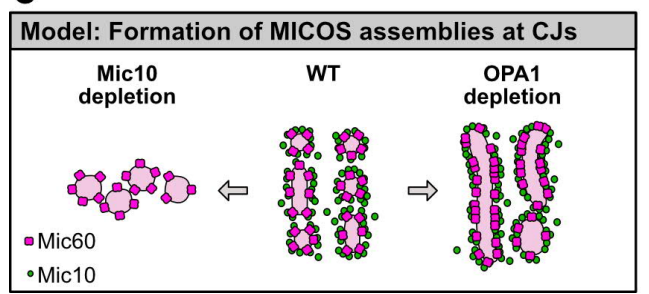

D

\begin{tabular}{|c|c|c|c|}
\hline & cristae shape & Mic60 distribution & crista junctions \\
\hline WT & - lamellar & $\begin{array}{l}\text { - clusters or } \\
\text { small rods } \\
\text { - often arranged in } \\
\text { transverse stripes }\end{array}$ & $\begin{array}{l}\text { - circular or short } \\
\text { slit-like CJs } \\
\text { - often arranged in } \\
\text { transverse stripes }\end{array}$ \\
\hline $\begin{array}{c}\text { Mic10 } \\
\text { depletion }\end{array}$ & - tube-like & $\begin{array}{l}\text { - clusters } \\
\text { - arranged in two } \\
\text { opposite bands }\end{array}$ & $\begin{array}{l}\text { - number reduced } \\
\text { - circular shape } \\
\text { - diameter enlarged }\end{array}$ \\
\hline $\begin{array}{c}\text { Mic60 } \\
\text { depletion }\end{array}$ & $\begin{array}{l}\text { - tube-like } \\
\text { - onion-shaped } \\
\text { - stacks }\end{array}$ & - no Mic60 present & $\begin{array}{l}\text { - number strongly } \\
\text { reduced }\end{array}$ \\
\hline $\begin{array}{c}\text { OPA1 } \\
\text { depletion }\end{array}$ & $\begin{array}{l}\text { - lamellar } \\
\text { (disordered) } \\
\text { - septa }\end{array}$ & $\begin{array}{l}\text { - extended Mic60 } \\
\text { assemblies }\end{array}$ & $\begin{array}{l}\text { - slit-like CJs } \\
\text { - in addition septa } \\
\text { junctions }\end{array}$ \\
\hline $\begin{array}{c}\text { OPA1 \& Mic10 } \\
\text { depletion }\end{array}$ & $\begin{array}{l}\text { - tube-like } \\
\text { - vesicular } \\
\text { - septa }\end{array}$ & $\begin{array}{l}\text { - clusters } \\
\text { - scattered } \\
\text { distribution }\end{array}$ & $\begin{array}{l}\text { - number } \\
\text { strongly reduced } \\
\text { - in addition septa } \\
\text { junctions }\end{array}$ \\
\hline $\begin{array}{c}\text { OPA1 \& Mic60 } \\
\text { depletion }\end{array}$ & $\begin{array}{l}\text { - tube-like } \\
\text { - onion-shaped } \\
\text { - stacks } \\
\text { - septa }\end{array}$ & - no Mic60 present & $\begin{array}{l}\text { - number } \\
\text { strongly reduced } \\
\text { - in addition septa } \\
\text { junctions }\end{array}$ \\
\hline
\end{tabular}

Fig 10. Summary of findings and model of MICOS-controlled lamellar crista formation. (A) Model for the formation of crista membranes (CMs) in WT, Mic10-KO and Mic60-KO cells. Shown are cartoons of longitudinal cross sections of mitochondria. For details see main text. Right lower corner: Model for the localizations of the key membrane shaping proteins involved in lamellar cristae formation at a lamellar crista in WT cells. Shown is a transversal cross section through a mitochondrial tubule (view on a single crista). The CM is displayed in blue. (B) Illustration of the Mic60 redistribution upon re-expression of Mic10 in Mic10 depleted mitochondria. (C) Model of the Mic10- and OPA1-dependent formation of MICOS assemblies at CJs. (D) Table summarizing the phenotypes that were observed in this study upon the depletion of key players in cristae formation. 


\section{Material and Methods}

Materials availability. Further information and requests for resources and reagents should be directed to the corresponding author, Stefan Jakobs (sjakobs@gwdg.de).

Cell culture and transfection. HeLa cells (Gruber, Lampe et al., 2005) were grown in Dulbecco's Modified Eagle Medium (DMEM) with glutaMAX and 4.5 g/L glucose (Thermo Fisher Scientific, Waltham, MA, USA) supplemented with $100 \mathrm{U} / \mathrm{mL}$ penicillin and $100 \mu \mathrm{g} / \mathrm{mL}$ streptomycin (Merck Millipore, Burlington, MA, USA), $1 \mathrm{mM}$ sodium pyruvate (Sigma Aldrich, Munich, Germany) and $10 \%$ (v/v) fetal bovine serum (Merck Millipore) at $37{ }^{\circ} \mathrm{C}$ and $5 \% \mathrm{CO}_{2}$. For gene silencing by RNA interference (RNAi), cells were transfected with siRNA pools (siTOOLs Biotech, Planegg, Germany) according to the manufacturer's instruction using the Lipofectamine RNAiMAX transfection reagent (Thermo Fisher Scientific). Plasmid transfections were carried out using jetPRIME (Polyplus-transfection SA, IllkirchGraffenstaden, France) or FuGENE HD (Promega, Fitchburg, WI, USA).

Generation of knockout (KO) cell lines by CRISPR/Cas9. Sequence information about each target gene was collected from the gene database of the National Center for Biotechnology Information (NCBI). Each gRNA was designed using the CRISPR design tool from Benchling based on the scoring models from (Doench, Fusi et al., 2016, Hsu, Scott et al., 2013). For the cloning of the nuclease plasmids, the expression vector PX458 was digested with the BbSI restriction endonuclease (New England Biolabs, Ipswich, MA, USA) and purified. Oligonucleotides were hybridized and ligated into PX458. pSpCas9(BB)-2A-GFP (PX458) was a gift from Feng Zhang (Addgene plasmid \# 48138; http://n2t.net/addgene:48138; RRID:Addgene_48138). HeLa cells were transfected with the respective nuclease plasmid and cells expressing Cas9-EGFP were sorted using a BD Influx cell sorter (BD Biosciences, Flanklin Lakes, NJ , USA) 4 days after transfection. After clonal expansion, the single cell clones were analyzed by SDS-PAGE and Western blotting. Gene disruption was verified by PCR of the target gene, sub-cloning and sequencing.

\section{Oligonucleotides for knockout generation and analysis.}

\begin{tabular}{|ll|}
\hline Oligonucleotide & Sequence (5‘3`) \\
gRNA for Mic10-KO FW & CACCGTGTCTGAGTCGGAGCTCGGC \\
gRNA for Mic10-KO REV & AAACGCCGAGCTCCGACTCAGACAC \\
gRNA for Mic13-KO FW & CACCGGCTGGGGGCGCCGTCTACC \\
gRNA for Mic13-KO REV & AAACGGTAGACGGCGCCCCCAGCC \\
gRNA for Mic19-KO FW & CACCGCGAGAATGAGAACATCACCG \\
gRNA for Mic19-KO REV & AAACCGGTGATGTTCTCATTCTCGC \\
gRNA for Mic25-KO FW & CACCGTCTACCTTTGGCCTTCAAGA \\
\hline
\end{tabular}


Chapter 4 - Role of MICOS in Cristae Formation

\begin{tabular}{|ll|}
\hline gRNA for Mic25-KO REV & AAACTCTTGAAGGCCAAAGGTAGAC \\
gRNA for Mic26-KO FW & CACCGTCACTCTACTCAGTTCCTGA \\
gRNA for Mic26-KO REV & AAACTCAGGAACTGAGTAGAGTGAC \\
gRNA for Mic27-KO FW & CACCGACTGCAACTGGTTGTTACAT \\
gRNA for Mic27-KO REV & AAACATGTAACAACCAGTTGCAGTC \\
gRNA for Mic60-KO FW & CACCGCTGCGGGCCTGTCAGTTAT \\
gRNA for Mic60-KO REV & AAACATAACTGACAGGCCCGCAGC \\
Analysis Primer Mic10-KO FW & GGTGAGGAGGAAAGGCCTGGTCACG \\
Analysis Primer Mic10-KO REV & TTCCACTCAAGAGCTCTGCGACTC \\
Analysis Primer Mic13-KO FW & CAGTTCATCAGTTCAAGTGGCGTCCAGCC \\
Analysis Primer Mic13-KO REV & TTACCTGCATTCCAGGAGTCACGGATGG \\
Analysis Primer Mic19-KO FW & GAAAAGAATCCAGGCCCTTCCACGCGC \\
Analysis Primer Mic19-KO REV & CAGTGCCTAGCACTTGGCACAACCAGGAA \\
Analysis Primer Mic25-KO FW & CTCAGCATGGACCTGGTAGGCACTGGGC \\
Analysis Primer Mic25-KO REV & GCCTCAATTCCCACATGGAGAAAGTGGC \\
Analysis Primer Mic26-KO FW & TAAAGTTCAGGTTGCTTGTAACCCTTAGAGTCA \\
Analysis Primer Mic26-KO REV & TATCAAATAGGTTTTATTCATTCTTGCTACTTGC \\
Analysis Primer Mic27-KO FW & CCCCAAAGGATCCATTTTACTGTGGATGGAC \\
Analysis Primer Mic27-KO REV & TCCCAGCTGAACCCAGTCATCCAGCCATCC \\
Analysis Primer Mic60-KO FW & CCTCCGGCAGTGTTCACCTAGTAACCCCTT \\
Analysis Primer Mic60 KO REV & TCGCCCGTCGACCTTCAGCACTGAAAACCTAT \\
\hline &
\end{tabular}

\section{Generation of stable Mic10 and Mic60 TetOn (TO) cell lines by CRISPR/Cas9}

pTRE-Tight-Mic60. The plasmid pTRE-Tight-EGFP-donor fw copy was linearized by PCR. Mic60 was amplified by PCR and both fragments were ligated by Gibson Assembly (New England Biolabs). pTRE-Tight-EGFP-donor fw copy was a gift from Rudolf Jaenisch (Addgene plasmid \# 22074; http://n2t.net/addgene:22074; RRID:Addgene_22074).

AAVS1-TRE3G-Mic10-FLAG-T2A-EGFP. The plasmid AAVS1-TRE3G-EGFP was linearized by the restriction endonuclease SalI. Mic10-FLAG was amplified by PCR, thereby also introducing a C-terminal T2A self-cleavage site. Fragments were ligated by Gibson Assembly (New England Biolabs). AAVS1-TRE3G-EGFP was a gift from Su-Chun Zhang (Addgen plasmid \#52343; http://n2t.net/addgene:52343; RRID:Addgene_52343).

PX-330-AAVS1. The nuclease plasmid PX330-AAVS1 was derived from PX330. In brief, oligonucleotides were annealed by primer annealing and integrated into PX330 after linearization with the BbSI restriction endonuclease. pX330-U6-Chimeric_BB-CBh-hSpCas9 was a gift from Feng Zhang (Addgene plasmid \# 42230; http://n2t.net/addgene:42230; RRID:Addgene_42230). 
Oligonucleotides for plasmids.

\begin{tabular}{|ll|}
\hline Oligonucleotide & Sequence $\mathbf{( 5}^{`} \mathbf{- 3}^{`}$ ) \\
pTRE-Tight FW & CCAGAGTGAGATATCTCTAGAGGATCATAATCAGC \\
pTRE-Tight REV & CCGCAGCATGGTGGCGGCGGAATCTCCAGGCGATCTG \\
Mic60 FW & GCCGCCACCATGCTGCGGGCCTGTCAGTT \\
Mic60 REV & AGAGATATCTCACTCTGGCTGCACCTGAG \\
Mic10-FLAG FW & TCCTACCCTCGTAAAGATATCGCCGCCACCATGTCTGAGTCGGA \\
Mic10-FLAG REV & GCTC \\
& ACCTTGCTCACCATGTCGACTGGGCCGGGATTCTCCTCCACGTC \\
& CGTCATCCTTG \\
AAVS1-gRNA FW & CACCGTGTCCCTAGTGGCCCCACTG \\
AAVS1-gRNA REV & AAACCAGTGGGGCCACTAGGGACAC \\
\hline
\end{tabular}

Integration into the AAVS1 safe harbor locus. For generation of Mic60-TO cells, the donor plasmids pTRE-Tight-Mic60 and AAVS1-SA-2A-NEO-CAG-RTTA3 were co-transfected with the plasmid PX330-AAVS1 into Mic60-KO HeLa cells. Starting 2 days after transfection, cells were selected with 1.25-1.5 $\mu \mathrm{g} / \mathrm{mL}$ puromycin (InvivoGen, San Diego, CA, USA) for 3 days. Cells were cultivated without antibiotics for 1day and afterwards selected with DMEM containing $800 \mu \mathrm{g} / \mathrm{mL}$ G418 (Carl Roth, Karlsruhe, Germany) for 8 days. After a recovery period of 10 days, single cell clones were obtained using a BD Influx cell sorter (BD Biosciences). After clonal expansion, positive clones were detected by PCR and analyzed for Mic60 expression by Western blotting and immunofluorescence staining using a specific antiserum against Mic60 (Proteintech, Rosemont, IL, USA). The plasmid AAVS1-SA-2ANEO-CAG-RTTA3 was a gift from Paul Gadue (Addgene plasmid \# 6043; http://n2t.net/addgene:60431; RRID:Addgene_60431).

Oligonucleotides for integration analysis.

\begin{tabular}{|ll|}
\hline Oligonucleotide & Sequence $\left(5^{`} \mathbf{3}^{`}\right)$ \\
Analyze AAVS1 WT FW & CCCCTATGTCCACTTCAGGA \\
Analyze AAVS1 WT REV & CAGCTCAGGTTCTGGGAGAG \\
Analyze TRE FW & CATTTTTTCACTGCCTCGACAGTACTAAGC \\
Analyze TRE REV & GAAGGATGCAGGACGAGAAA \\
Analyze CAG FW & TGAATTCACTCCTCAGGTGCAGGCTGCCTAT \\
Analyze CAG REV & GAAGGATGCAGGACGAGAAA \\
\hline
\end{tabular}

To generate Mic10-TO cells, AAVS1-TRE3G-Mic10-FLAG-T2A-EGFP and PX330-AAVS1 were co-transfected into Mic10-KO HeLa cells. 2 days after transfection, cells were selected with $1.25-1.5 \mu \mathrm{g} / \mathrm{mL}$ puromycin (Invivogen) for 3 days. After 10 days, cells were induced with $1 \mu \mathrm{g} / \mathrm{mL}$ doxycycline hyclate (Sigma Aldrich) for $24 \mathrm{~h}$ and cells expressing EGFP were sorted using a BD Influx cell sorter (BD Biosciences). After clonal expansion, Mic10-FLAG 
expression was verified by Western blotting and immunofluorescence staining using specific antisera against the FLAG-tag (Sigma Aldrich) and Mic10 (abcam, Cambridge, UK).

Induction of Mic10-FLAG or Mic60 expression in Mic10-TO and Mic60-TO cells. To avoid unintended induction, Mic10-TO and Mic60-TO cells were generally cultivated in DMEM containing tetracycline-free FBS (TAKARA BIO INC., Kusatsu, Japan). To induce expression of Mic10-FLAG or Mic60, the medium was supplemented with doxycycline hyclate (Sigma Aldrich) at a concentration of $0.025 \mu \mathrm{g} / \mathrm{mL}$ (Mic10-TO cells) or $0.25 \mu \mathrm{g} / \mathrm{mL}$ (Mic60-TO cells) for up to $72 \mathrm{~h}$.

Transient knockdowns. Knockdowns of Mic10, Mic13, Mic19, Mic25, Mic26, Mic27, Mic60, OPA1 or ATP5ME were achieved by transfection with the respective siRNA pool (siTOOLs Biotech). Cells were cultivated for 2-5 days after transfection.

Knockdown of DRP1 was achieved by transfection with the shRNA expression plasmid pREP4 (Lee et al., 2004). After transfection, cells were selected with DMEM supplemented with 250 $\mu \mathrm{M}$ hygromycin B (Life Technologies, Carlsbad, USA) for 2 days. Afterwards, cells were selected for 5 days with $50 \mu \mathrm{M}$ hygromycin B (Life technologies). All knockdowns were verified by Western blotting and immunofluorescence microscopy.

Transient expression of Mic10-SNAP. The Mic10-SNAP expression plasmid pH-MINOS1SNAP was produced by Gateway reaction of pSEMS-GATEWAY-26m (Covalys Biosciences, Witterswil, Switzerland) and pCR8-MINOS1 (Human ORFeome cDNA clone collection V5.1, Open BioSystems Inc, Huntsville, AL, USA)

Transient expression of COX8A-SNAP. Transient expression of COX8-SNAP was achieved by transfection with AAVS1-Blasticidin-CAG-COX8A-SNAP, as detailed elsewhere (Stephan et al., 2019).

Real-time respirometry. Oxygen consumption rate (OCR) experiments were performed in an XF Extracellular Flux Analyzer (Seahorse Bioscience, Billerica, MA, USA) as previously described (Pacheu-Grau, Wasilewski et al., 2020). Briefly, HeLa cells were seeded at 25,000 cells/well and grown on the Seahorse plate overnight. Baseline respiration was measured in XF DMEM supplemented with $1 \mathrm{mM}$ pyruvate and $10 \mathrm{mM}$ glucose and $2 \mathrm{mM}$ Glutamine after incubation at $37^{\circ} \mathrm{C}$ in an incubator without $\mathrm{CO}_{2}$ for $1 \mathrm{~h}$. Periodic oxygen consumption measurements were performed, and OCR was calculated from the slope of change in oxygen concentration over time. Metabolic states were measured after subsequent addition of $3 \mu \mathrm{M}$ oligomycin, $1 \mu \mathrm{M}$ carbonyl cyanide 4 (trifluoromethoxy)phenylhydrazone (FCCP), $1 \mu \mathrm{M}$ antimycin $\mathrm{A}$, and $2 \mu \mathrm{M}$ rotenone. For normalization, cell density was calculated using CyQUANT ${ }^{\circledR}$ after OCR measurements, according to manufacturer's instructions by measuring 
fluorescence intensity (Ex $480 \mathrm{~nm}$, Em $520 \mathrm{~nm})$. OCR values (N=6) were normalized to cell density (ratio of WT) and presented as \% of WT.

Isolation of mitochondria from cultured human cells. Mitochondria were isolated after cell homogenization by differential centrifugation, essentially as previously described (Callegari, Richter et al., 2016).

BN-PAGE. Mitochondria were solubilized in $1 \%$ digitonin, $20 \mathrm{mM}$ Tris-HCl, $\mathrm{pH}$ 7.4, $0.1 \mathrm{mM}$ EDTA, $50 \mathrm{mM} \mathrm{NaCl}, 10 \%$ (w/v) glycerol, $1 \mathrm{mM}$ phenylmethylsulfonyl fluoride for $30 \mathrm{~min}$ at $4{ }^{\circ} \mathrm{C}$. Unsoluble material was removed by centrifugation at $20,000 \mathrm{xg}$ and $4{ }^{\circ} \mathrm{C}$ for $15 \mathrm{~min}$. After addition of 10x loading dye (5\% Coomassie brilliant blue G-250, $500 \mathrm{mM} \varepsilon$-amino ncapronic acid, $100 \mathrm{mM}$ Bis-Tris, $\mathrm{pH}$ 7.0), the supernatant was loaded on 4-13\% polyacrylamide gradient gels and separated as described before (Wittig, Braun et al., 2006).

Affinity purification of protein complexes. For immunoprecipitation of Mic60, the corresponding antibody was coupled to protein A sepharose (GE Healtcare, Chicago, IL, USA) using dimethyl pimelilidate according to the manufactory protocol. WT and MICOS mutant mitochondria were solubilized in a buffer containing $1 \%$ digitonin, $20 \mathrm{mM}$ Tris- $\mathrm{HCl}, \mathrm{pH} 7.4$, $1 \mathrm{mM}$ EDTA, $100 \mathrm{mM} \mathrm{NaCl}, 10 \%$ (w/v) glycerol, $1 \mathrm{mM}$ phenylmethylsulfonyl fluoride for $1 \mathrm{~h}$ at $4{ }^{\circ} \mathrm{C}$. Non-solubilized material was removed by centrifugation at $20.000 \mathrm{xg}$ and $4{ }^{\circ} \mathrm{C}$ for $15 \mathrm{~min}$ and the supernatant was mixed with beads. After $1 \mathrm{~h}$ binding at $4{ }^{\circ} \mathrm{C}$, the beads were washed with $0.3 \%$ digitonin buffer containing $20 \mathrm{mM}$ Tris-HCl, $\mathrm{pH}$ 7.4, $1 \mathrm{mM}$ EDTA, 100 $\mathrm{mM} \mathrm{NaCl}, 10 \%$ (w/v) glycerol, $1 \mathrm{mM}$ phenylmethylsulfonyl fluoride. Bound material was eluted with $100 \mathrm{mM}$ glycine $\mathrm{pH} 2.8$ at room temperature (RT) for $5 \mathrm{~min}$.

For analysis of Mic10-TO cells, whole cells induced with doxycycline hyclate for 8, 16 or $24 \mathrm{~h}$ as well as non-induced cells were solubilized in a buffer containing $1 \%$ digitonin, $20 \mathrm{mM}$ Tris$\mathrm{HCl}, \mathrm{pH}$ 7.4, $1 \mathrm{mM}$ EDTA, $100 \mathrm{mM} \mathrm{NaCl}, 10 \%$ (w/v) glycerol, $1 \mathrm{mM}$ phenylmethylsulfonyl fluoride for $1 \mathrm{~h}$ at $4{ }^{\circ} \mathrm{C}$. Non-solubilized material was removed by centrifugation at $20.000 \mathrm{xg}$ and $4{ }^{\circ} \mathrm{C}$ for $15 \mathrm{~min}$. The supernatant was either incubated with FLAG-beads (Sigma Aldrich) or Mic60-Beads for $1 \mathrm{~h}$ at $4{ }^{\circ} \mathrm{C}$. The beads were washed with $0.3 \%$ digitonin buffer containing $20 \mathrm{mM}$ Tris-HCl, pH 7.4, $1 \mathrm{mM}$ EDTA, $100 \mathrm{mM} \mathrm{NaCl}, 10 \%$ (w/v) glycerol, $1 \mathrm{mM}$ phenylmethylsulfonyl fluoride. Bound material was eluted with $100 \mathrm{mM}$ glycine pH 2.8 at RT for $5 \mathrm{~min}$.

\section{Antibodies for Western blot analysis.}

\begin{tabular}{|ll|}
\hline Epitope & Source \\
Mic10 & abcam (Cambridge, UK) and Callegari et al., 2019 \\
Mic13 & Sigma Aldrich and Callegari et al., 2019 \\
Mic19 & Atlas Antibodies (Bromma, Sweden) and Callegari et al., 2019 \\
Mic25 & Proteintech (Rosemont, IL, USA) and Callegari et al., 2019 \\
\hline
\end{tabular}




\begin{tabular}{|ll|}
\hline Mic26 & Thermo Fisher Scientific and Callegari et al., 2019 \\
Mic27 & Atlas Antibodies and Callegari et al., 2019 \\
Mic60 & Proteintech and Callegari et al., 2019 \\
ATPB & Molecular Probes (Eugene, OR, USA) \\
ATP5B & Callegari et al., 2019 \\
ATP5A & abcam \\
ATP5ME & Proteintech \\
OPA1 (D7C1A) & Cell Signaling Technology (Danvers, MA, USA) \\
DRP1 & BD Biosciences (San Jose, CA, USA) \\
MFN1 (D6E2S) & Cell Signaling Technology \\
MFN2 (D2D10) & Cell Signaling Technology \\
RIESKE & Callegari et al., 2019 \\
COX1 & Callegari et al., 2019 \\
LETM1 & Callegari et al., 2019 \\
SDHA & Callegari et al., 2019 \\
NDUFA10 & Callegari et al., 2019 \\
\hline
\end{tabular}

Sample preparation for fluorescence microscopy. For immunolabelling, cells were cultured on coverslips for $1-2$ days at $37{ }^{\circ} \mathrm{C}$ with $5 \% \mathrm{CO}_{2}$ and fixed with prewarmed $\left(37{ }^{\circ} \mathrm{C}\right)$ $4 \%$ or $8 \%$ formaldehyde in PBS $\left(137 \mathrm{mM} \mathrm{NaCl}, 2.68 \mathrm{mM} \mathrm{KCl}\right.$ and $10 \mathrm{mM} \mathrm{Na} 2 \mathrm{HPO}_{4}, \mathrm{pH}$ 7.4) for 5-10 min at RT. Fixed cells were extracted with $0.5 \%(\mathrm{v} / \mathrm{v})$ Triton-X-100 in PBS, blocked with $5 \%(\mathrm{w} / \mathrm{v})$ BSA in PBS and incubated with diluted primary antibodies against Mic60 (Proteintech), Mic19 (Atlas Antibodies), TOM20 (Santa Cruz Biotechnology, Dallas, TX, USA), ATPB (abcam, Cambridge, UK), FLAG (Sigma Aldrich), or dsDNA (abcam) in 5 $\%(\mathrm{w} / \mathrm{v}) \mathrm{BSA}$ in PBS for $1 \mathrm{~h}$ at RT. After washing in PBS, the primary antibodies were detected with secondary goat anti-rabbit or sheep anti-mouse antibodies labeled with Alexa Fluor 594 (Thermo Fisher Scientific) or custom-labeled with Abberior STAR RED (Dye: Abberior, Göttingen, Germany; antibody: Jackson Immuno Research Laboratories, West Grove, PA, USA) in $5 \%$ (w/v) BSA in PBS for $1 \mathrm{~h}$ at RT. After washing with PBS, the cells were mounted in Mowiol with $0.1 \%$ 1,4-Diazabicyclo[2.2.2] octan (DABCO) and $2.5 \mu \mathrm{g} / \mathrm{mL}$ 4',6-Diamidin2-phenylindol (DAPI) (Sigma Aldrich).

For live-cell imaging of COX8A-SNAP or Mic10-SNAP fusion proteins, cells were seeded in glass bottom dishes (ibidi GmbH, Martinsried, Germany) before the measurements. Cells were stained with DMEM containing $1 \mu \mathrm{M}$ SNAP-Cell SiR (New England Biolabs) and $0.1 \%$ (v/v) Quant-IT PicoGreen dsDNA reagent (Thermo Fisher Scientific) for 15-20 min. The staining solution was removed and the cells were washed with DMEM twice. Cells were left in the incubator for about 20 min to remove unbound dye. For imaging, the DMEM was replaced with live-cell imaging solution (Thermo Fisher Scientific). Cells were recorded by stimulated emission depletion (STED) microscopy. For live cell imaging of mitochondrial membranes, cells were seeded in glass bottom dishes (ibidi GmbH, Martinsried, Germany) and stained with DMEM containing $125 \mathrm{nM}$ Mitotracker Green (Thermo Fisher Scientific) for 15-20 min. Cells 
were washed twice with DMEM and incubated for about 15 min to remove unbound dye. Medium was replaced by live-cell imaging solution (Thermo Fisher Scientific) and cells were recorded with 3D linear structured illumination microscopy (3D SIM). For STED nanoscopy of Mic60 together with COX8A-SNAP, cells expressing COX8A-SNAP were stained with SNAP-cell SiR as described above and subsequently fixed by adding 2x PHEM buffer supplemented with $4.8 \%$ formaldehyde and $0.2 \%$ glutaraldehyde in equal amount to the culture medium for 25 minutes at RT. Samples were permeabilized with $0.05 \%$ (v/v) Triton X-100 in PHEM buffer for $5 \mathrm{~min}$. To remove free glutaraldehyde, the samples were incubated in $0.1 \mathrm{M}$ ammonium chloride in PHEM for 1 minute and afterwards were blocked with PHEM containing $1 \% \mathrm{BSA}$ and $0.2 \%$ saponin for 10 minutes. For immunolabeling, the primary antibody was diluted in blocking solution and incubated over night at $4^{\circ} \mathrm{C}$. Washing was performed 5 times with blocking solution. Secondary antibodies were diluted in blocking solution and applied for $2 \mathrm{~h}$ at room temperature. The sample was washed with blocking solution 5 times and imaged in PHEM buffer.

Light microscopy. Confocal microscopy was performed with a Leica TCS SP8 (Leica, Wetzlar, Germany). STED nanoscopy was performed using dual-color Abberior STED 775 QUAD scanning microscopes (Abberior Instruments, Göttingen, Germany) with either a 775nm Katana-08 HP laser (Onefive GmbH, Regensdorf, Switzerland) or a 775nm STEDLaser from Abberior Instruments. In brief, for immunolabeled samples the fluorophore Alexa Fluor 594 was exited at $561 \mathrm{~nm}$ or $594 \mathrm{~nm}$ and Abberior STAR RED was exited at $640 \mathrm{~nm}$. STED was performed at $775 \mathrm{~nm}$. Images were recorded with a pixel size of $15-20 \mathrm{~nm}$ in the 2D STED mode and with a voxel size of $50 \mathrm{~nm}$ in 3D STED. For live-cell STED microscopy, SNAP-cell SiR was excited at $640 \mathrm{~nm}$ and depletion was performed at $775 \mathrm{~nm}$. Images were recorded with a pixel size of 20-25nm. EGFP or PicoGreen were exited at $488 \mathrm{~nm}$ and recorded in the confocal mode. The used objective was an UPlanSApo 100x/1.40 Oil [infinity]/0,17 / FN26.5 objective (Olympus, Tokyo, Japan).

3D structured illumination microscopy of living HeLa cells was performed with a commercial Deltavision OMXv4.0 BLAZE microscope (GE Healthcare, Amersham, UK) using a 60x, 1.42 NA oil immersion PlanApoN objective lens (Olympus) and sCMOS cameras. MitoTracker Green was excited at $488 \mathrm{~nm}$ and the emission recorded at 504-552 nm. The intensities and exposure times were set to obtain satisfactory signal strength. A sequence of 15 images for each axial plane, obtained at three different angles with five phases each, was acquired. Multiple axial planes encompassing the entire cell from top to bottom were recorded at a separation of the individual axial planes of $125 \mathrm{~nm}$.

3D MINFLUX nanoscopy was performed with a custom-built MINFLUX nanoscope that was described previously (Gwosch et al., 2020). For active stabilization of the sample, coverslips were covered with gold nano rods (Nanopartz Inc., Loveland, CO, USA). Nano rods were diluted 1:3 in single molecule clean phosphate-buffered saline (Sigma Aldrich) and sonicated for 5-10 min. The samples were incubated with the nano rod solution for 5-10 min at RT. The 
sample was washed 3 times with PBS. For MINFLUX imaging, a standard enzyme-based (d)STORM blinking buffer containing $50 \mathrm{nM}$ TRIS/HCl pH 8.0, $10 \mathrm{mM} \mathrm{NaCl}, 10 \%$ (w/v) glucose, supplemented with $0.4 \mathrm{mg} / \mathrm{mL}$ glucose oxidase (Sigma Aldrich) and $90 \mathrm{mM}$ cysteamine hydrochloride (Sigma Aldrich) was used. Samples were sealed using picodent twinsil speed 22 (picodent ${ }^{\circledR}$ Dental-Produktions- und Vertriebs-GmbH, Wipperfürth, Germany). The imaging was performed with parameters and imaging schemes as reported previously (Gwosch et al., 2020). Briefly, before MINFLUX measurements, the fluorophore Alexa Fluor 647 was transferred into a long-lived non-fluorescent state by excitation at $642 \mathrm{~nm}$ wavelength. Conditional photo-activation of single molecules in the MINFLUX region was performed by illumination at $405 \mathrm{~nm}$ wavelength. For localization, molecules were excited with displaced Gaussian or 3D doughnut-shaped excitation beams at $642 \mathrm{~nm}$ wavelength. Fluorescence photons were collected in a confocal detection. Scanning of the activation laser and the MINFLUX targeted coordinate pattern was performed in steps of $250 \mathrm{~nm}$ in a customshaped region selected based on fluorescence widefield images.

\section{Image processing and analysis}

Image processing for confocal and STED microscopy. Unless stated in the figure legend, image raw data were not deconvolved, but smoothed with a low-pass filter using the Imspector Software (Abberior Instruments). When deconvolution was applied, we relied on the Richardson-Lucy algorithm and the Imspector software (Abberior Instruments).

In all images the color tables were adapted for optimal contrast. Background subtraction was usually below $5 \%$ of the maximum signal intensity.

Image processing for 3D SIM. Super-resolved fluorescence images were reconstructed with the corresponding recorded optical transfer function (OTF) in the softWoRx 7.0.0 software (GE Healthcare, Amersham, UK) at a Wiener filter setting of 0.006 .

Image processing for MINFLUX nanoscopy. Data analysis and evaluation was performed as described previously (Gwosch et al., 2020). False-positive localizations due to reaction to background or molecular emission events far outside the MINFLUX region were removed based on p_0 $<0.11$ and r_relative $<32 \mathrm{~nm}$. To ensure taking into account only emission events from single molecules (i.e. not from groups of simultaneously activated molecules) localizations with high photon count rates $(>100 \mathrm{kHz})$ were discarded. Localizations with photon numbers above 1000 and an estimated signal-to-background value larger than 0.6 were selected to guarantee high localization precisions.

Cluster analysis on STED images of OPA1 depleted cells. Analysis of the clustered fraction of Mic60 was performed by a custom written Matlab script. Every image was filtered with a Laplacian of Gaussian (80 $\mathrm{nm}$ FWHM) filter and segmented with a threshold of $4 \%$ of the brightest value. Segments with an area smaller than $0.0225 \mu \mathrm{m}^{2}$ were defined as single 
clusters. The clustered fraction is the ratio of the area of all single cluster segments relative to the total segmented area.

Sample preparation for electron microscopy. Aclar discs were punched with $18 \mathrm{~mm}$ diameter using $0.198 \mathrm{~mm}$ thick aclar film (Plano, Wetzlar, Germany) and sterilized with $70 \%$ ethanol before usage. Cells were grown on aclar discs to a confluency of $\sim 70 \%$ and fixed by immersion using $2 \%$ glutaraldehyde in $0.1 \mathrm{M}$ cacodylate buffer at $\mathrm{pH} 7.4$ for $1 \mathrm{~h}$ at RT. Fixation was completed overnight at $4{ }^{\circ} \mathrm{C}$. After post-fixation in $1 \%$ osmium tetroxide and preembedding staining with $1 \%$ uranyl acetate, samples were dehydrated and embedded in Agar 100 resin (Plano, Wetzlar, Germany). For FIB-SEM, cells were grown on 6 x 0.16 mm sapphire discs (Wohlwend $\mathrm{GmbH}$, Sennwald $\mathrm{CH}$ ) and vitrified using a a Leica EM HPM100 high pressure freezer (Leica Mikrosysteme Vertriebe $\mathrm{GmbH}$, Wetzlar, Germany). The frozen samples were transferred to an automatic freeze substitution unit Leica EM AFS2 (Leica Mikrosysteme Vertrieb $\mathrm{GmbH}$ ) and substituted at $-90{ }^{\circ} \mathrm{C}$ for $4 \mathrm{~h}$ in a solution containing anhydrous acetone, $2 \%$ osmium tetroxide (EMS Electron Microscopical Science, Ft. Washington, USA), $0.1 \%$ uranyl acetate in acetone and $5 \%$ dest. $\mathrm{H}_{2} \mathrm{O}$. After gradually warm up to $0{ }^{\circ} \mathrm{C}$, samples were washed with acetone and embedded using Durcupan resin (Science Services GmbH, München, Germany).

Transmission electron microscopy (TEM) and electron tomography (ET). Ultrathin sections of $\sim 70 \mathrm{~nm}$ thickness were recorded on a Philips CM 120 BioTwin transmission electron microscope (Philips Inc., Eindhoven, The Netherlands) without counterstaining. Usually, 2D images of at least 100 mitochondria from at least 10 different cells were randomly taken for each sample, using a TemCam 224A slow scan CCD camera (TVIPS, Gauting, Germany).

For electron tomography, tilt series from $210 \mathrm{~nm}$ thick sections from Agar100 embedded cells were recorded on a Talos L120C transmission microscope (Thermo Fischer Scientific/ FEI company, Hilsboro, Oregon, USA) at 17,500x magnification using a Ceta 4kx4k CMOS camera in unbinning mode. Orthogonal series were recorded from $-64.5^{\circ}$ to $64.5^{\circ}$ using $3^{\circ}$ saxton angular increase. The series were calculated using Etomo (David Mastronade, http://bio3d.colorado.edu/). Tomograms were processed using the nonlinear anisotropic diffusion (NAD) filter in imod. Recordings of thin sections were processed in Fiji using the median filter.

Focused ion beam scanning electron microscopy. Polymerized samples were trimmed with a razor blade, removing empty resin. The sapphire disks were removed and the tip of the block containing the cells was sawed off with a jigsaw (Villinger, Gregorius et al., 2012). The blocks were attached to the SEM stub (Science Services GmbH, Pin $12.7 \mathrm{~mm}$ x $3.1 \mathrm{~mm}$ ) by a silver filled epoxy (Epoxy Conductive Adhesive, EPO-TEK EE 129-4; EMS) and polymerized at $60^{\circ}$ overnight. The samples were coated with a $10 \mathrm{~nm}$ gold, platinum or platinum/palladium 
layer using the sputter coating machine EM ACE600 (Leica Mikrosysteme Vertrieb GmbH) at $35 \mathrm{~mA}$ current. The samples were placed into the Crossbeam 540 focused ion beam scanning electron microscope (Carl Zeiss Microscopy GmbH, Oberkochen, Germany). The SmartSEM software (Carl Zeiss Microscopy $\mathrm{GmbH}$ ) was used to deposit a $400 \mathrm{~nm}$ platinum layer on top of the region of interest using a $3 \mathrm{nA}$ current, to ensure even milling and to protect the surface. Then a trench was milled to expose a cross-section through the cell of interest using $15 \mathrm{nA}$ current. The cross-section was polished using a $7 \mathrm{nA}$ current. The Atlas 3D (Atlas 5.1, Fibics, Canada) software was used to collect the 3D data. The images were acquired at $1.5 \mathrm{kV}$ (analytic mode) with the ESB detector (450 V ESB grid, pixel size $\mathrm{x} / \mathrm{y} 5 \mathrm{~nm}$ ) in a continuous mill and acquire mode. A $700 \mathrm{pA}$ current was applied to remove $5 \mathrm{~nm}$ in between every image. Data post-processing steps were performed in Fiji (Schindelin, Arganda-Carreras et al., 2012). Image alignments were done using the "Linear Stack Alignment SIFT". The dataset was cropped, inverted, a Gaussian blur (1) and a local contrast enhancement (CLAHE; blocksize 127, histogram bins 100, maximum slope 1.5) were applied.

Segmentation and 3D animation of FIB-SEM and ET data. FIB-SEM and ET data sets were segmented using the software package IMOD (David Mastronade, http://bio3d.colorado.edu/imod/). 3D reconstructions of ET data were animated using Amira for Life Sciences (Thermo Fisher Scientific). Reconstructions of FIB-SEM data were animated using Blender (Blender Foundation, Amsterdam, The Netherlands).

Visualization of MINFLUX data. Data were visualized using Imaris (Bitplane, Belfast, UK). 


\section{Data Availability}

All raw data are available from the corresponding author upon reasonable request.

\section{Acknowledgements}

We thank Jan Keller-Findeisen for support with data analysis, Rita Schmitz-Salue for excellent technical assistance and Jaydev Jethwa for a careful reading of the manuscript. This work was supported by the European Research Council (ERCAdG No. 835102) (to SJ) and by the German Research Foundation-funded FOR2848 (project P08 to WM and Z01 to DR) and SFB1190 (project P01 to SJ and P13 to PR).

\section{Author Contributions}

S.J. and T.S. conceived the project. T.S., C.B., M.D., P.R., D.R. and S.J. designed research. T.S., C.B., M.D., A.M.S., M.B., T.S.B, G.H., W.H., F.L., D. P.-G. and S.S. performed research. T.S., C.B., M.D., A.M.S., F.B., T.S.B, P.I., J.P., T.H., S.W.H., W.M., P.R., D.R. and S.J. analyzed data. T.S. and S.J. wrote the paper with comments from all authors.

\section{Conflict of Interest}

The authors declare no conflict of interests. 


\section{References}

Alexander C, Votruba M, Pesch UE, Thiselton DL, Mayer S, Moore A, Rodriguez M, Kellner U, Leo-Kottler B, Auburger G, Bhattacharya SS, Wissinger B (2000) OPA1, encoding a dynamin-related GTPase, is mutated in autosomal dominant optic atrophy linked to chromosome 3q28. Nat Genet 26: 211-5

Alkhaja AK, Jans DC, Nikolov M, Vukotic M, Lytovchenko O, Ludewig F, Schliebs W, Riedel D, Urlaub H, Jakobs S, Deckers M (2012) MINOS1 is a conserved component of mitofilin complexes and required for mitochondrial function and cristae organization. Molecular Biology of the Cell 23: 247-257

Anand R, Strecker V, Urbach J, Wittig I, Reichert AS (2016) Mic13 Is Essential for Formation of Crista Junctions in Mammalian Cells. PLoS One 11: e0160258

Anand R, Wai T, Baker MJ, Kladt N, Schauss AC, Rugarli E, Langer T (2014) The i-AAA protease YME1L and OMA1 cleave OPA1 to balance mitochondrial fusion and fission. $\mathbf{J}$ Cell Biol 204: 919-29

Arnold I, Pfeiffer K, Neupert W, Stuart RA, Schagger H (1998) Yeast mitochondrial F1F0ATP synthase exists as a dimer: identification of three dimer-specific subunits. EMBO $\mathrm{J}$ 17: $7170-8$

Ban-Ishihara R, Ishihara T, Sasaki N, Mihara K, Ishihara N (2013) Dynamics of nucleoid structure regulated by mitochondrial fission contributes to cristae reformation and release of cytochrome c. Proc Natl Acad Sci U S A 110: 11863-8

Barbot M, Jans DC, Schulz C, Denkert N, Kroppen B, Hoppert M, Jakobs S, Meinecke M (2015) Mic10 oligomerizes to bend mitochondrial inner membranes at cristae junctions. Cell Metab 21: 756-63

Barrera M, Koob S, Dikov D, Vogel F, Reichert AS (2016) OPA1 functionally interacts with MIC60 but is dispensable for crista junction formation. FEBS Lett 590: 3309-3322

Bleazard W, McCaffery JM, King EJ, Bale S, Mozdy A, Tieu Q, Nunnari J, Shaw JM (1999) The dynamin-related GTPase Dnm1 regulates mitochondrial fission in yeast. Nature Cell Biology 1: 298-304

Blum TB, Hahn A, Meier T, Davies KM, Kuhlbrandt W (2019) Dimers of mitochondrial ATP synthase induce membrane curvature and self-assemble into rows. Proc Natl Acad Sci U S A

Bohnert M, Zerbes RM, Davies KM, Muhleip AW, Rampelt H, Horvath SE, Boenke T, Kram A, Perschil I, Veenhuis M, Kuhlbrandt W, van der Klei IJ, Pfanner N, van der Laan M (2015) Central role of Mic10 in the mitochondrial contact site and cristae organizing system. Cell Metab 21: 747-55

Callegari S, Muller T, Schulz C, Lenz C, Jans DC, Wissel M, Opazo F, Rizzoli SO, Jakobs S, Urlaub H, Rehling P, Deckers M (2019) A MICOS-TIM22 Association Promotes Carrier Import into Human Mitochondria. J Mol Biol 
Callegari S, Richter F, Chojnacka K, Jans DC, Lorenzi I, Pacheu-Grau D, Jakobs S, Lenz C, Urlaub H, Dudek J, Chacinska A, Rehling P (2016) TIM29 is a subunit of the human carrier translocase required for protein transport. FEBS Lett 590: 4147-4158

Cereghetti GM, Stangherlin A, Martins de Brito O, Chang CR, Blackstone C, Bernardi P, Scorrano L (2008) Dephosphorylation by calcineurin regulates translocation of Drp1 to mitochondria. Proc Natl Acad Sci U S A 105: 15803-8

Chan DC (2012) Fusion and fission: interlinked processes critical for mitochondrial health. Annu Rev Genet 46: 265-87

Cipolat S, Martins de Brito O, Dal Zilio B, Scorrano L (2004) OPA1 requires mitofusin 1 to promote mitochondrial fusion. Proc Natl Acad Sci U S A 101: 15927-32

Cogliati S, Enriquez JA, Scorrano L (2016) Mitochondrial Cristae: Where Beauty Meets Functionality. Trends Biochem Sci 41: 261-73

Darshi M, Mendiola VL, Mackey MR, Murphy AN, Koller A, Perkins GA, Ellisman MH, Taylor SS (2011) ChChd3, an inner mitochondrial membrane protein, is essential for maintaining crista integrity and mitochondrial function. J Biol Chem 286: 2918-32

Davies KM, Anselmi C, Wittig I, Faraldo-Gomez JD, Kuhlbrandt W (2012) Structure of the yeast F1Fo-ATP synthase dimer and its role in shaping the mitochondrial cristae. Proc Natl Acad Sci U S A 109: 13602-7

Delettre C, Lenaers G, Griffoin JM, Gigarel N, Lorenzo C, Belenguer P, Pelloquin L, Grosgeorge J, Turc-Carel C, Perret E, Astarie-Dequeker C, Lasquellec L, Arnaud B, Ducommun B, Kaplan J, Hamel CP (2000) Nuclear gene OPA1, encoding a mitochondrial dynamin-related protein, is mutated in dominant optic atrophy. Nat Genet 26: 207-10

Doench JG, Fusi N, Sullender M, Hegde M, Vaimberg EW, Donovan KF, Smith I, Tothova Z, Wilen C, Orchard R, Virgin HW, Listgarten J, Root DE (2016) Optimized sgRNA design to maximize activity and minimize off-target effects of CRISPR-Cas9. Nat Biotechnol 34: 184-191

Dudkina NV, Sunderhaus S, Braun HP, Boekema EJ (2006) Characterization of dimeric ATP synthase and cristae membrane ultrastructure from Saccharomyces and Polytomella mitochondria. FEBS Lett 580: 3427-32

Eydt K, Davies KM, Behrendt C, Wittig I, Reichert AS (2017) Cristae architecture is determined by an interplay of the MICOS complex and the F1FO ATP synthase via Mic27 and Mic10. Microb Cell 4: 259-272

Faelber K, Dietrich L, Noel JK, Wollweber F, Pfitzner AK, Muhleip A, Sanchez R, Kudryashev M, Chiaruttini N, Lilie H, Schlegel J, Rosenbaum E, Hessenberger M, Matthaeus C, Kunz S, von der Malsburg A, Noe F, Roux A, van der Laan M, Kuhlbrandt W et al. (2019) Structure and assembly of the mitochondrial membrane remodelling GTPase Mgm1. Nature 571: 429-433

Frezza C, Cipolat S, Martins de Brito O, Micaroni M, Beznoussenko GV, Rudka T, Bartoli D, Polishuck RS, Danial NN, De Strooper B, Scorrano L (2006) OPA1 controls apoptotic cristae remodeling independently from mitochondrial fusion. Cell 126: 177-89 
Friedman JR, Mourier A, Yamada J, McCaffery JM, Nunnari J (2015) MICOS coordinates with respiratory complexes and lipids to establish mitochondrial inner membrane architecture. Elife 4

Friedman JR, Nunnari J (2014) Mitochondrial form and function. Nature 505: 335-43

Giacomello M, Pyakurel A, Glytsou C, Scorrano L (2020) The cell biology of mitochondrial membrane dynamics. Nat Rev Mol Cell Biol

Glytsou C, Calvo E, Cogliati S, Mehrotra A, Anastasia I, Rigoni G, Raimondi A, Shintani N, Loureiro M, Vazquez J, Pellegrini L, Enriquez JA, Scorrano L, Soriano ME (2016) Optic Atrophy 1 Is Epistatic to the Core MICOS Component MIC60 in Mitochondrial Cristae Shape Control. Cell Rep 17: 3024-3034

Gruber J, Lampe T, Osborn M, Weber K (2005) RNAi of FACE1 protease results in growth inhibition of human cells expressing lamin A: implications for Hutchinson-Gilford progeria syndrome. J Cell Sci 118: 689-96

Guarani V, McNeill EM, Paulo JA, Huttlin EL, Frohlich F, Gygi SP, Van Vactor D, Harper JW (2015) QIL1 is a novel mitochondrial protein required for MICOS complex stability and cristae morphology. Elife 4

Gwosch KC, Pape JK, Balzarotti F, Hoess P, Ellenberg J, Ries J, Hell SW (2020) MINFLUX nanoscopy delivers 3D multicolor nanometer resolution in cells. Nat Methods 17: 217-224

Habersetzer J, Larrieu I, Priault M, Salin B, Rossignol R, Brethes D, Paumard P (2013) Human F1F0 ATP synthase, mitochondrial ultrastructure and OXPHOS impairment: a (super)complex matter? PLoS One 8: e75429

Hackenbrock CR (1966) Ultrastructural bases for metabolically linked mechanical activity in mitochondria. I. Reversible ultrastructural changes with change in metabolic steady state in isolated liver mitochondria. J Cell Biol 30: 269-97

Harner M, Korner C, Walther D, Mokranjac D, Kaesmacher J, Welsch U, Griffith J, Mann M, Reggiori F, Neupert W (2011) The mitochondrial contact site complex, a determinant of mitochondrial architecture. EMBO J 30: 4356-70

Harner ME, Unger AK, Geerts WJ, Mari M, Izawa T, Stenger M, Geimer S, Reggiori F, Westermann B, Neupert W (2016) An evidence based hypothesis on the existence of two pathways of mitochondrial crista formation. Elife 5

Harner ME, Unger AK, Izawa T, Walther DM, Ozbalci C, Geimer S, Reggiori F, Brugger B, Mann M, Westermann B, Neupert W (2014) Aim24 and MICOS modulate respiratory function, tafazzin-related cardiolipin modification and mitochondrial architecture. Elife 3: $\mathrm{e} 01684$

Hessenberger M, Zerbes RM, Rampelt H, Kunz S, Xavier AH, Purfurst B, Lilie H, Pfanner N, van der Laan M, Daumke O (2017) Regulated membrane remodeling by Mic60 controls formation of mitochondrial crista junctions. Nat Commun 8: 15258

Hoppins S, Collins SR, Cassidy-Stone A, Hummel E, Devay RM, Lackner LL, Westermann B, Schuldiner M, Weissman JS, Nunnari J (2011) A mitochondrial-focused genetic 
interaction map reveals a scaffold-like complex required for inner membrane organization in mitochondria. J Cell Biol 195: 323-40

Hsu PD, Scott DA, Weinstein JA, Ran FA, Konermann S, Agarwala V, Li Y, Fine EJ, Wu X, Shalem O, Cradick TJ, Marraffini LA, Bao G, Zhang F (2013) DNA targeting specificity of RNA-guided Cas9 nucleases. Nat Biotechnol 31: 827-32

Ishihara N, Eura Y, Mihara K (2004) Mitofusin 1 and 2 play distinct roles in mitochondrial fusion reactions via GTPase activity. J Cell Sci 117: 6535-46

Ishihara N, Fujita Y, Oka T, Mihara K (2006) Regulation of mitochondrial morphology through proteolytic cleavage of OPA1. EMBO J 25: 2966-77

Janer A, Prudent J, Paupe V, Fahiminiya S, Majewski J, Sgarioto N, Des Rosiers C, Forest A, Lin ZY, Gingras AC, Mitchell G, McBride HM, Shoubridge EA (2016) SLC25A46 is required for mitochondrial lipid homeostasis and cristae maintenance and is responsible for Leigh syndrome. EMBO Mol Med 8: 1019-38

Jans DC, Wurm CA, Riedel D, Wenzel D, Stagge F, Deckers M, Rehling P, Jakobs S (2013) STED super-resolution microscopy reveals an array of MINOS clusters along human mitochondria. Proc Natl Acad Sci U S A 110: 8936-8941

Jiang YF, Lin SS, Chen JM, Tsai HZ, Hsieh TS, Fu CY (2017) Electron tomographic analysis reveals ultrastructural features of mitochondrial cristae architecture which reflect energetic state and aging. Sci Rep 7: 45474

Kojima R, Kakimoto Y, Furuta S, Itoh K, Sesaki H, Endo T, Tamura Y (2019) Maintenance of Cardiolipin and Crista Structure Requires Cooperative Functions of Mitochondrial Dynamics and Phospholipid Transport. Cell Rep 26: 518-528 e6

Kondadi AK, Anand R, Reichert AS (2019) Functional Interplay between Cristae Biogenesis, Mitochondrial Dynamics and Mitochondrial DNA Integrity. Int J Mol Sci 20

Kuhlbrandt W (2019) Structure and Mechanisms of F-Type ATP Synthases. Annu Rev Biochem 88: 515-549

Lee YJ, Jeong SY, Karbowski M, Smith CL, Youle RJ (2004) Roles of the mammalian mitochondrial fission and fusion mediators Fis1, Drp1, and Opa1 in apoptosis. Molecular Biology of the Cell 15: 5001-5011

MacVicar T, Langer T (2016) OPA1 processing in cell death and disease - the long and short of it. J Cell Sci 129: 2297-306

Meeusen S, DeVay R, Block J, Cassidy-Stone A, Wayson S, McCaffery JM, Nunnari J (2006) Mitochondrial inner-membrane fusion and crista maintenance requires the dynaminrelated GTPase Mgm1. Cell 127: 383-95

Muhleip AW, Joos F, Wigge C, Frangakis AS, Kuhlbrandt W, Davies KM (2016) Helical arrays of U-shaped ATP synthase dimers form tubular cristae in ciliate mitochondria. Proc Natl Acad Sci U S A 113: 8442-7

Nunnari J, Suomalainen A (2012) Mitochondria: in sickness and in health. Cell 148: 1145-59 
Olichon A, Baricault L, Gas N, Guillou E, Valette A, Belenguer P, Lenaers G (2003) Loss of OPA1 perturbates the mitochondrial inner membrane structure and integrity, leading to cytochrome c release and apoptosis. J Biol Chem 278: 7743-6

Pacheu-Grau D, Wasilewski M, Oeljeklaus S, Gibhardt CS, Aich A, Chudenkova M, Dennerlein S, Deckers M, Bogeski I, Warscheid B, Chacinska A, Rehling P (2020) COA6 facilitates cytochrome $\mathrm{c}$ oxidase biogenesis as thiol-reductase for copper metallochaperones in mitochondria. J Mol Biol

Palade GE (1952) The fine structure of mitochondria. Anat Rec 114: 427-51

Patten DA, Wong J, Khacho M, Soubannier V, Mailloux RJ, Pilon-Larose K, MacLaurin JG, Park DS, McBride HM, Trinkle-Mulcahy L, Harper ME, Germain M, Slack RS (2014) OPA1-dependent cristae modulation is essential for cellular adaptation to metabolic demand. EMBO J 33: 2676-91

Paumard P, Vaillier J, Coulary B, Schaeffer J, Soubannier V, Mueller DM, Brethes D, di Rago JP, Velours J (2002) The ATP synthase is involved in generating mitochondrial cristae morphology. EMBO Journal 21: 221-230

Pernas L, Scorrano L (2016) Mito-Morphosis: Mitochondrial Fusion, Fission, and Cristae Remodeling as Key Mediators of Cellular Function. Annu Rev Physiol 78: 505-31

Pfanner N, van der Laan M, Amati P, Capaldi RA, Caudy AA, Chacinska A, Darshi M, Deckers M, Hoppins S, Icho T, Jakobs S, Ji J, Kozjak-Pavlovic V, Meisinger C, Odgren PR, Park SK, Rehling P, Reichert AS, Sheikh MS, Taylor SS et al. (2014) Uniform nomenclature for the mitochondrial contact site and cristae organizing system. J Cell Biol 204: 1083-6

Quintana-Cabrera R, Quirin C, Glytsou C, Corrado M, Urbani A, Pellattiero A, Calvo E, Vazquez J, Enriquez JA, Gerle C, Soriano ME, Bernardi P, Scorrano L (2018) The cristae modulator Optic atrophy 1 requires mitochondrial ATP synthase oligomers to safeguard mitochondrial function. Nat Commun 9: 3399

Rabl R, Soubannier V, Scholz R, Vogel F, Mendl N, Vasiljev-Neumeyer A, Korner C, Jagasia R, Keil T, Baumeister W, Cyrklaff M, Neupert W, Reichert AS (2009) Formation of cristae and crista junctions in mitochondria depends on antagonism between Fcj1 and $\mathrm{Su}$ e/g. $\mathbf{J}$ Cell Biol 185: 1047-63

Ramonet D, Perier C, Recasens A, Dehay B, Bove J, Costa V, Scorrano L, Vila M (2013) Optic atrophy 1 mediates mitochondria remodeling and dopaminergic neurodegeneration linked to complex I deficiency. Cell Death Differ 20: 77-85

Rampelt H, Bohnert M, Zerbes RM, Horvath SE, Warscheid B, Pfanner N, van der Laan M (2017a) Mic10, a Core Subunit of the Mitochondrial Contact Site and Cristae Organizing System, Interacts with the Dimeric F1Fo-ATP Synthase. J Mol Biol 429: 1162-1170

Rampelt H, van der Laan M (2017) The Yin \& Yang of Mitochondrial Architecture - Interplay of MICOS and F1Fo-ATP synthase in cristae formation. Microb Cell 4: 236-239

Rampelt H, Zerbes RM, van der Laan M, Pfanner N (2017b) Role of the mitochondrial contact site and cristae organizing system in membrane architecture and dynamics. Biochim Biophys Acta 1864: 737-746 
Ryan MD, King AM, Thomas GP (1991) Cleavage of foot-and-mouth disease virus polyprotein is mediated by residues located within a 19 amino acid sequence. J Gen Virol 72 ( Pt 11): 2727-32

Scheffler IE (2008) Mitochondria, second edition. John Wiley \& Sons, Inc Hoboken, New Jersey, USA

Schindelin J, Arganda-Carreras I, Frise E, Kaynig V, Longair M, Pietzsch T, Preibisch S, Rueden C, Saalfeld S, Schmid B, Tinevez JY, White DJ, Hartenstein V, Eliceiri K, Tomancak P, Cardona A (2012) Fiji: an open-source platform for biological-image analysis. Nat Methods 9: 676-82

Sesaki H, Southard SM, Yaffe MP, Jensen RE (2003) Mgm1p, a dynamin-related GTPase, is essential for fusion of the mitochondrial outer membrane. Molecular Biology of the Cell 14: 2342-2356

Sjöstrand FS (1953) Electron microscopy of mitochondria and cytoplasmic double membranes. Nature 171: 30-2

Stephan T, Roesch A, Riedel D, Jakobs S (2019) Live-cell STED nanoscopy of mitochondrial cristae. Scientific Reports 9: 12419

Stoldt S, Stephan T, Jans DC, Bruser C, Lange F, Keller-Findeisen J, Riedel D, Hell SW, Jakobs S (2019) Mic60 exhibits a coordinated clustered distribution along and across yeast and mammalian mitochondria. Proc Natl Acad Sci U S A

Strauss M, Hofhaus G, Schroder RR, Kuhlbrandt W (2008) Dimer ribbons of ATP synthase shape the inner mitochondrial membrane. EMBO J 27: 1154-60

Tarasenko D, Barbot M, Jans DC, Kroppen B, Sadowski B, Heim G, Mobius W, Jakobs S, Meinecke M (2017) The MICOS component Mic60 displays a conserved membranebending activity that is necessary for normal cristae morphology. J Cell Biol 216: 889-899

van der Laan M, Horvath SE, Pfanner N (2016) Mitochondrial contact site and cristae organizing system. Curr Opin Cell Biol 41: 33-42

Varanita T, Soriano ME, Romanello V, Zaglia T, Quintana-Cabrera R, Semenzato M, Menabo R, Costa V, Civiletto G, Pesce P, Viscomi C, Zeviani M, Di Lisa F, Mongillo M, Sandri M, Scorrano L (2015) The OPA1-dependent mitochondrial cristae remodeling pathway controls atrophic, apoptotic, and ischemic tissue damage. Cell Metab 21: 834-44

Villinger C, Gregorius H, Kranz C, Hohn K, Munzberg C, von Wichert G, Mizaikoff B, Wanner G, Walther P (2012) FIB/SEM tomography with TEM-like resolution for 3D imaging of high-pressure frozen cells. Histochem Cell Biol 138: 549-56

von der Malsburg K, Muller JM, Bohnert M, Oeljeklaus S, Kwiatkowska P, Becker T, Loniewska-Lwowska A, Wiese S, Rao S, Milenkovic D, Hutu DP, Zerbes RM, SchulzeSpecking A, Meyer HE, Martinou JC, Rospert S, Rehling P, Meisinger C, Veenhuis M, Warscheid B et al. (2011) Dual role of mitofilin in mitochondrial membrane organization and protein biogenesis. Dev Cell 21: 694-707

Wai T, Langer T (2016) Mitochondrial Dynamics and Metabolic Regulation. Trends Endocrinol Metab 27: 105-117 
Wittig I, Braun HP, Schagger H (2006) Blue native PAGE. Nat Protoc 1: 418-28

Zanna C, Ghelli A, Porcelli AM, Karbowski M, Youle RJ, Schimpf S, Wissinger B, Pinti M, Cossarizza A, Vidoni S, Valentino ML, Rugolo M, Carelli V (2008) OPA1 mutations associated with dominant optic atrophy impair oxidative phosphorylation and mitochondrial fusion. Brain 131: 352-67

Zick M, Rabl R, Reichert AS (2009) Cristae formation-linking ultrastructure and function of mitochondria. Biochim Biophys Acta 1793: 5-19 


\section{Captions for Expanded View Movies}

Movie EV1. Cristae architecture of mitochondria from HeLa WT cells. Two mitochondria were reconstructed from a FIB-SEM stack. The cristae are shown in blue, the OM and IBM are shown together in clear grey. A twisted crista is highlighted in grey. A still image is shown in Fig 1B.

Movie EV2. Live-cell STED nanoscopy of Mic10-SNAP. Mic10-SNAP was expressed in HeLa cells and labeled with SNAP-cell SiR. Mitochondria were recorded with STED nanoscopy every 15 seconds. Photobleaching was compensated.

Movie EV3. ET of HeLa Mic60-KO cells. A mitochondrion was reconstructed from a tilt series recorded by TEM. The OM is displayed in clear grey, the side of the IM that faces the matrix is shown in dark blue. The IM side that faces the intermembrane space is shown in light blue. A still image in shown in Fig 3F.

Movie EV4. ET of HeLa Mic60-KO cells. Tilt series of mitochondria recorded by TEM.

Movie EV5. ET of HeLa WT cells. A mitochondrion was reconstructed from a tilt series recorded by TEM. The OM is displayed in clear grey, the side of the IM that faces the matrix is shown in dark blue. The IM side that faces the intermembrane space is shown in light blue. A still is shown in Fig 3F.

Movie EV6. ET of HeLa Mic10-KO cells. A mitochondrion was reconstructed from a tilt series recorded by TEM. The OM is displayed in clear grey, the side of the IM that faces the matrix is shown in dark blue. The IM side that faces the intermembrane space is shown in light blue. A still image is shown in Fig 3F.

Movie EV7. ET of HeLa Mic10-KO cells. Tilt series of a mitochondrion with an onion-shaped cristae architecture by TEM.

Movie EV 8. Live-cell STED nanoscopy of mitochondria from Mic10-KO cells. Cells expressing COX8A-SNAP were stained with SNAP-cell SiR and visualized by time-lapse STED nanoscopy every 5 seconds.

Movie EV9. Live-cell STED nanoscopy of mitochondria from Mic10-KO cells. Cells expressing COX8A-SNAP were stained with SNAP-cell SiR and visualized by time-lapse STED nanoscopy every 15 seconds. 
Movie EV10. FIB-SEM of Mic10-TO cells. Cristae architecture of mitochondria from a noninduced Mic10-TO cell. A mitochondrion was reconstructed from a FIB-SEM stack. The $\mathrm{CM}$ is shown in blue, the OM and IBM are shown together in clear grey. A still image is shown in Fig 7A.

Movie EV11. FIB-SEM of Mic10-TO cells. Cristae architecture of mitochondria from a Mic10TO cell induced for Mic10 re-expression for $16 \mathrm{~h}$. A mitochondrion was reconstructed from a FIB-SEM stack. The CM is shown in blue, the OM and IBM are shown together in clear grey. A still image is shown in Fig 7A.

Movie EV12. FIB-SEM of Mic10-TO cells. Cristae architecture of mitochondria from a Mic10TO cell induced for Mic10 re-expression for $24 \mathrm{~h}$. A mitochondrion was reconstructed from a FIB-SEM stack. The CM is shown in blue, the OM and IBM are shown together in clear grey. A still image is shown in Fig 7A.

Movie EV13. ET of Mic10-TO cells. A tilt series of a noninduced cell was recorded. Mitochondria were reconstructed. The OM is displayed in clear grey, the side of the IM that faces the matrix is shown in dark blue. The IM side that faces the intermembrane space is shown in light blue. A still image is shown in Fig 7B.

Movie EV14. ET of Mic10-TO cells. A tilt series was recorded of a cell induced for Mic10 reexpression for $16 \mathrm{~h}$. Mitochondria were reconstructed. The OM is displayed in clear grey, the side of the IM that faces the matrix is shown in dark blue. The IM side that faces the intermembrane space is shown in light blue. A still image is shown in Fig 7B.

Movie EV15. ET of HeLa control cells. Cells were transfected with a scrambled control for $48 \mathrm{~h}$. A tilt series was recorded and a mitochondrion was reconstructed. The OM is shown in grey, the IM in blue. The side facing the intermembrane space is shown in light blue and the side facing the matrix space is shown in dark blue. A still image is shown in Fig 8E.

Movie EV16. ET of HeLa OPA1-KD cells. Cells were transfected with siRNA pools against OPA1 for $48 \mathrm{~h}$. A tilt series was recorded and mitochondria were reconstructed. The OM is shown in grey, the IM in blue. The side facing the intermembrane space is shown in light blue and the side facing the matrix space is shown in dark blue. A still image is shown in Fig 8F. 


\section{Appendix}

\section{Appendix to \\ MICOS assembly controls mitochondrial inner membrane remodeling and crista junction redistribution to mediate cristae formation}

Till Stephan ${ }^{\mathrm{a}}$, Christian Brüser ${ }^{\mathrm{a}}$, Markus Deckers, Anna M. Steyer, Francisco Balzarotti, Mariam Barbot, Tiana S. Behr, Gudrun Heim, Wolfgang Hübner, Peter Ilgen, Felix Lange, David Pacheu-Grau, Jasmin Pape, Stefan Stoldt, Thomas Huser, Stefan W. Hell, Wiebke Möbius, Peter Rehling, Dietmar Riedel and Stefan Jakobs ${ }^{\mathrm{b}}$

${ }^{\text {a }}$ Equal contributions

${ }^{\mathrm{b}}$ To whom correspondence should be addressed (email: sjakobs@gwdg.de)

This appendix includes:

Appendix Figures S1 - S6

Appendix Table S1 
Chapter 4 - Role of MICOS in Cristae Formation

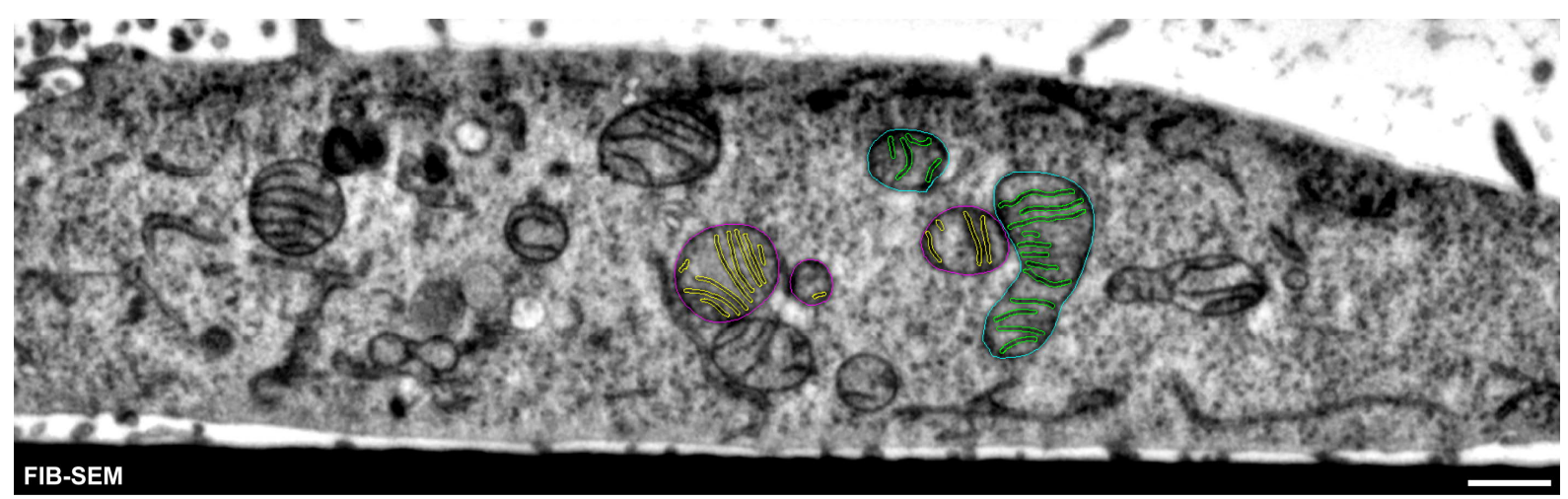

Appendix Fig S1. Inner membrane architecture in HeLa cells. (A) FIB-SEM of HeLa WT cells. Shown is one section of a FIB-SEM stack. Reconstructed mitochondria (colored lines) are shown in Fig 1B. Scale bar: $1 \mu \mathrm{m}$. 
A
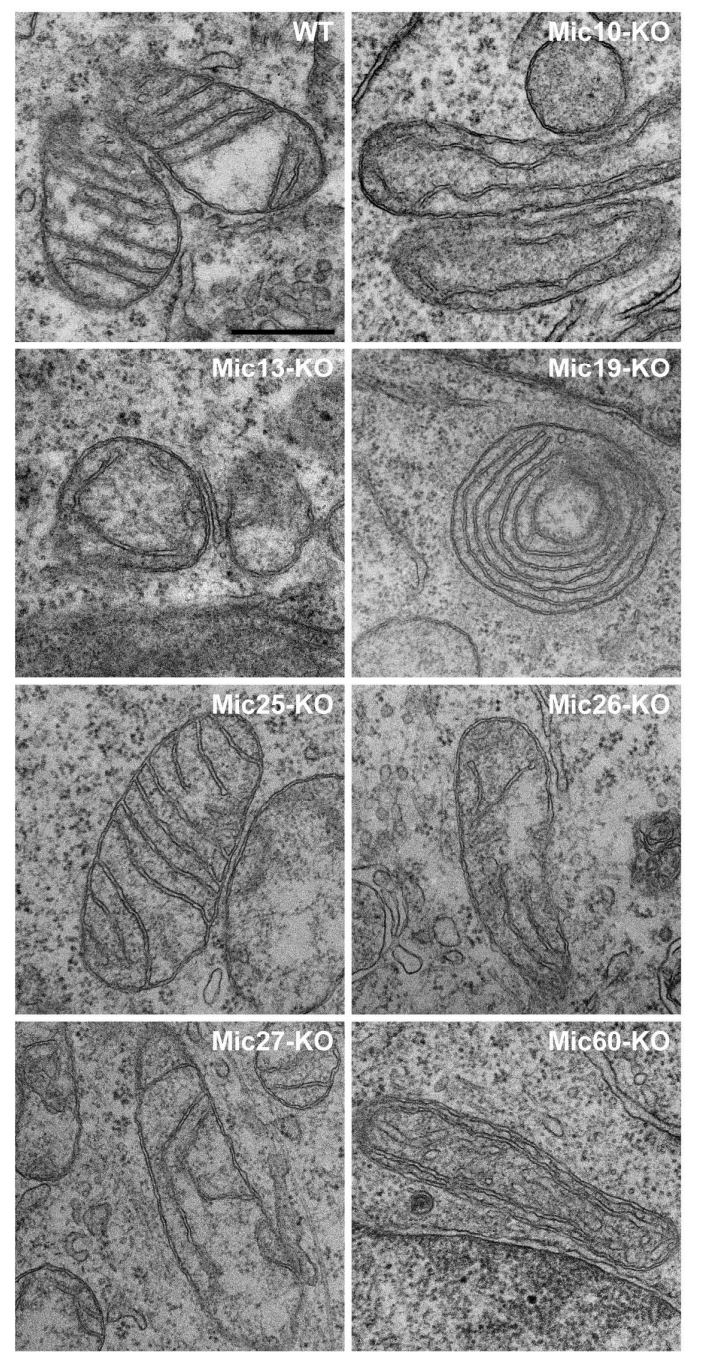

C

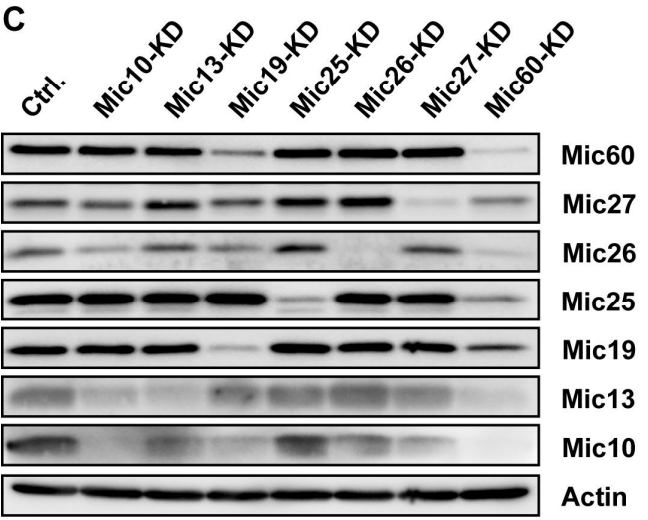

B
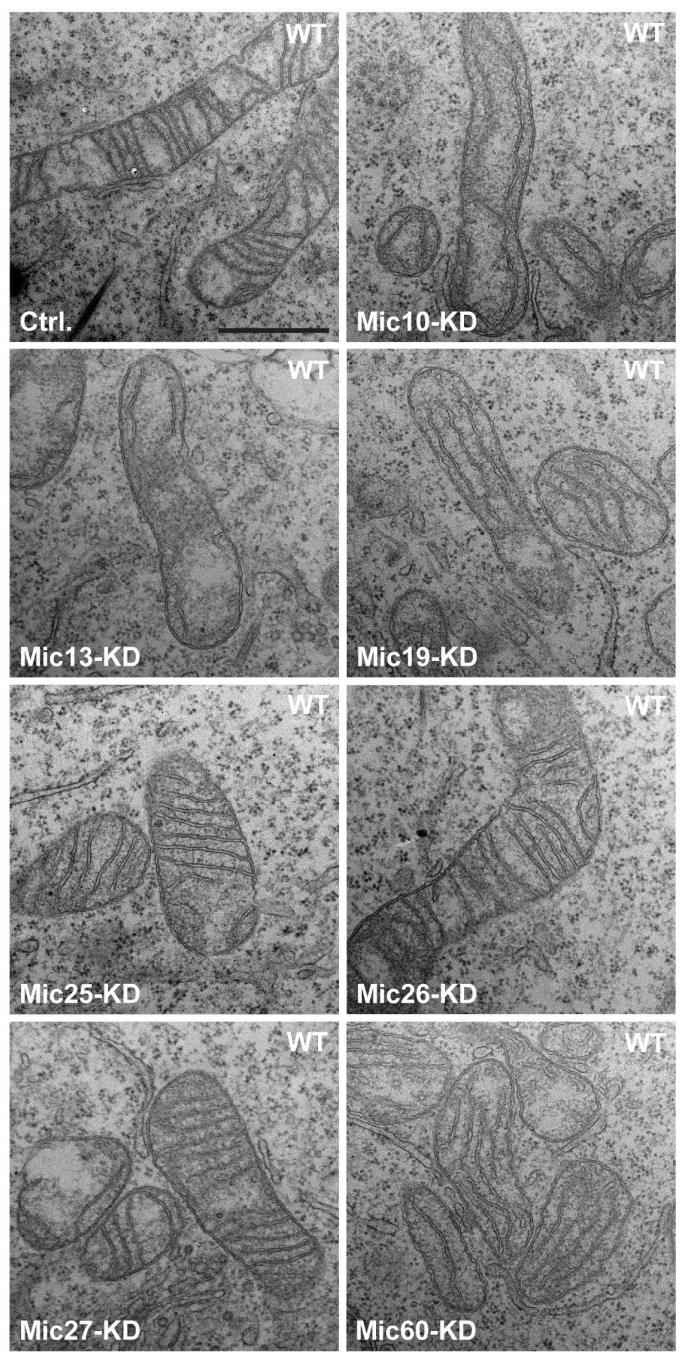

Appendix Fig S2. Depletion of MICOS subunits affects the formation of lamellar cristae. (A-B) Comparison of MICOS knockouts (KO) with MICOS knockdowns (KD). (A) Representative TEM recordings of the KO-cell lines indicated. (B,C) Knockdown of MICOS subunits using siRNA. WT cells were transfected with a scrambled control (Ctrl.) or specific siRNA pools and analyzed after $48 \mathrm{~h}$. (B) Images show representative TEM recordings. (C) Protein levels of MICOS subunits in cell lysates analyzed by Western blotting. Scale bars: $500 \mathrm{~nm}(\mathrm{~A}), 1 \mu \mathrm{m}(\mathrm{B})$. 
A
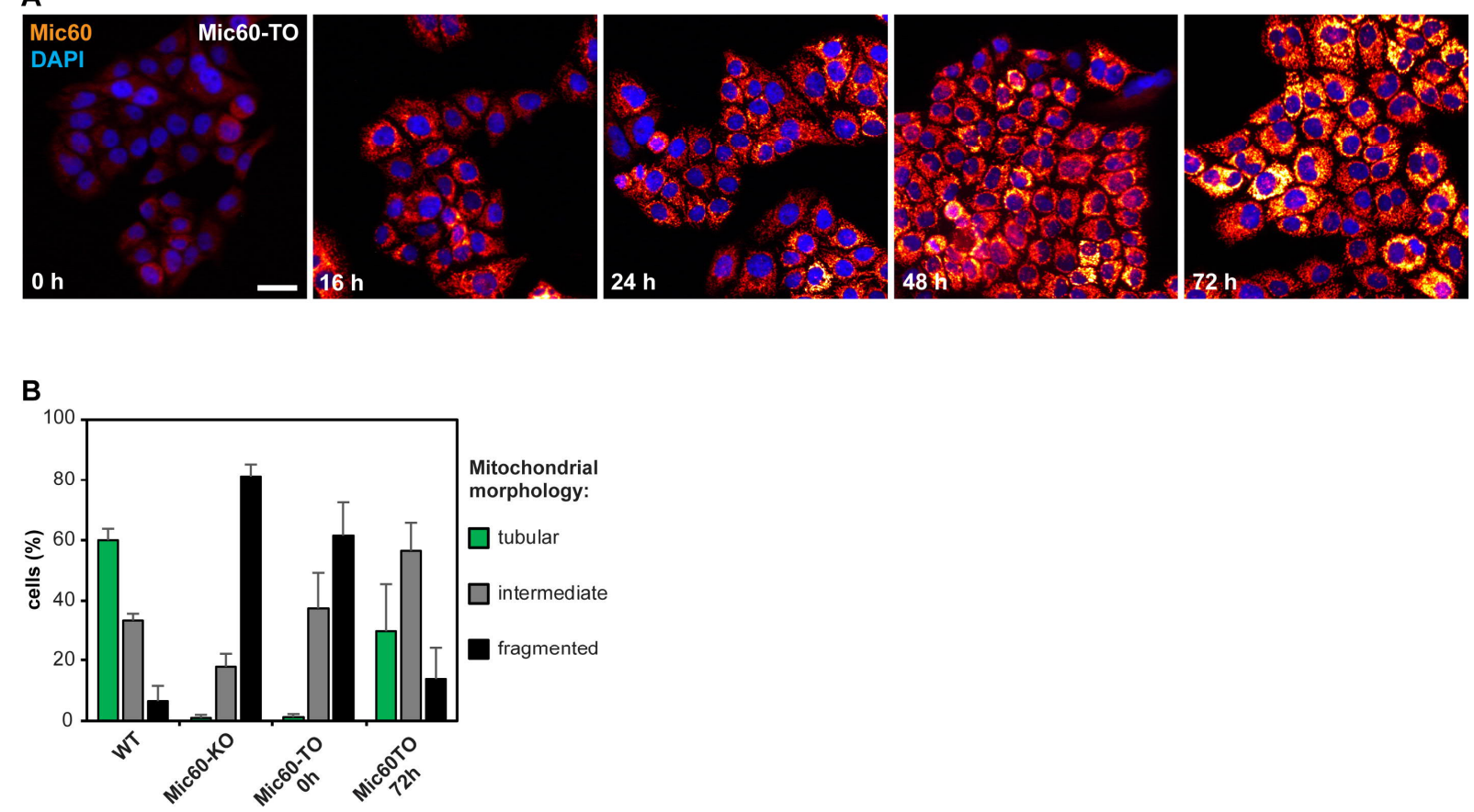

Appendix Fig S3. Re-expression of Mic60 in Mic60-TO cells rescues the morphology of the mitochondrial network. (A-B) Rescue of Mic60 expression in Mic60-TO cells upon induction with doxycycline. (A) Cells were immunolabeled for Mic60 and DNA and visualized by confocal fluorescence microscopy at the indicated time points after expression of Mic60. (B) Quantification of mitochondrial network remodeling upon Mic60 re-expression. For the analysis, cells were immunolabeled for TOM20, imaged by confocal fluorescence microscopy, and manually annotated. Average and SD of three independent biological repeats are shown. For each repeat, at least 170 cells per sample were analyzed. Scale bar: $40 \mu \mathrm{m}$. 

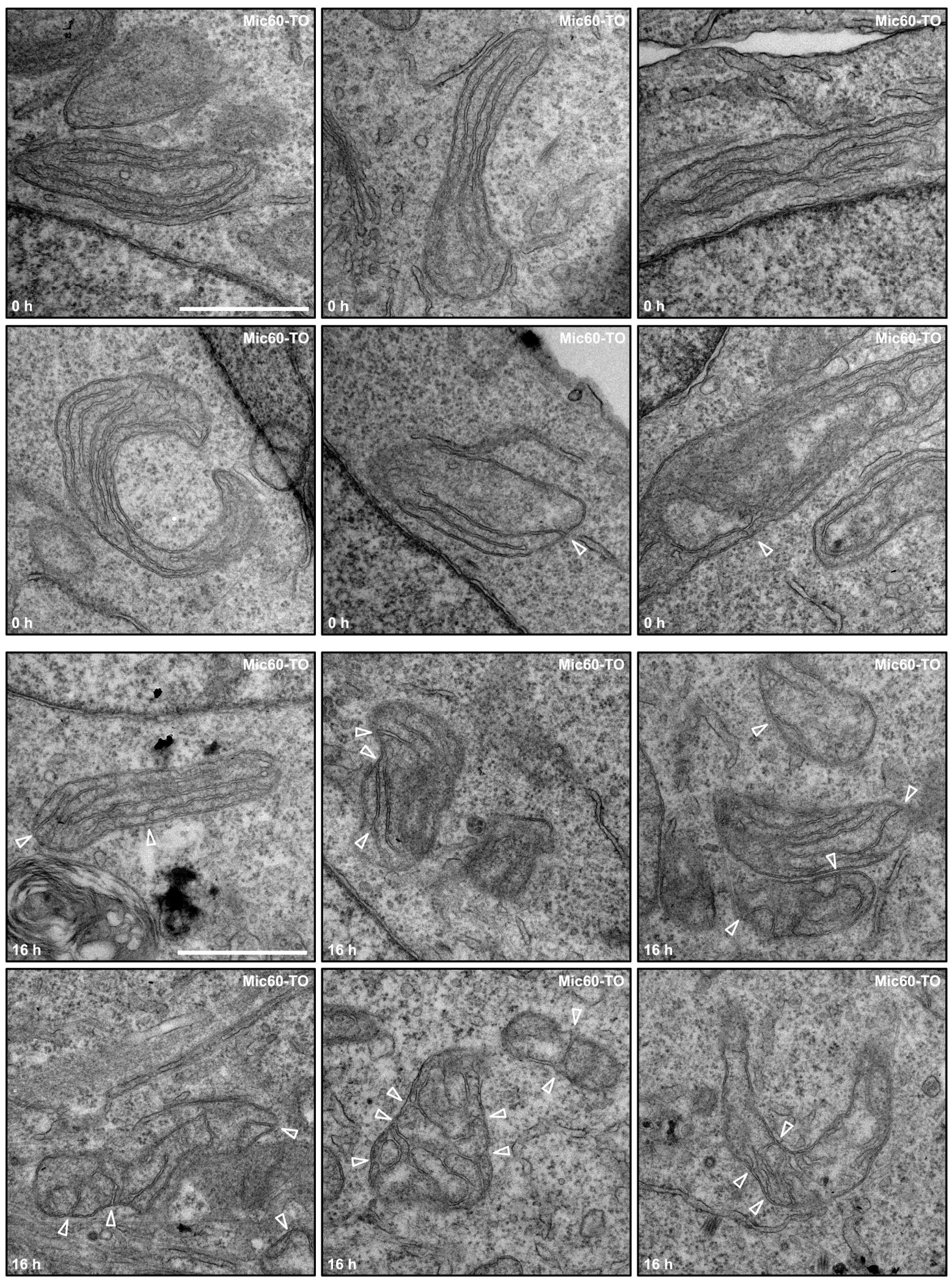

Appendix Fig S4. Mic60 controls the formation of secondary CJs. TEM recordings of Mic60-TO cells induced with doxycycline for $0 \mathrm{~h}$ and $16 \mathrm{~h}$ to facilitate the expression of Mic60. Arrows mark CJs. Scale bars: $1 \mu \mathrm{m}$. 
A

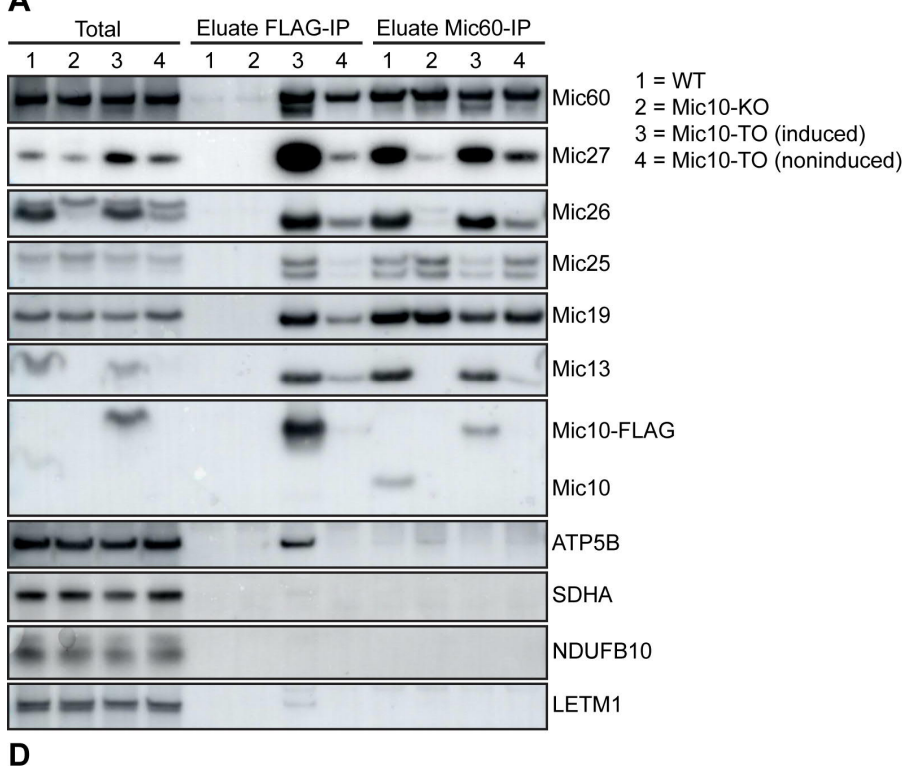

B

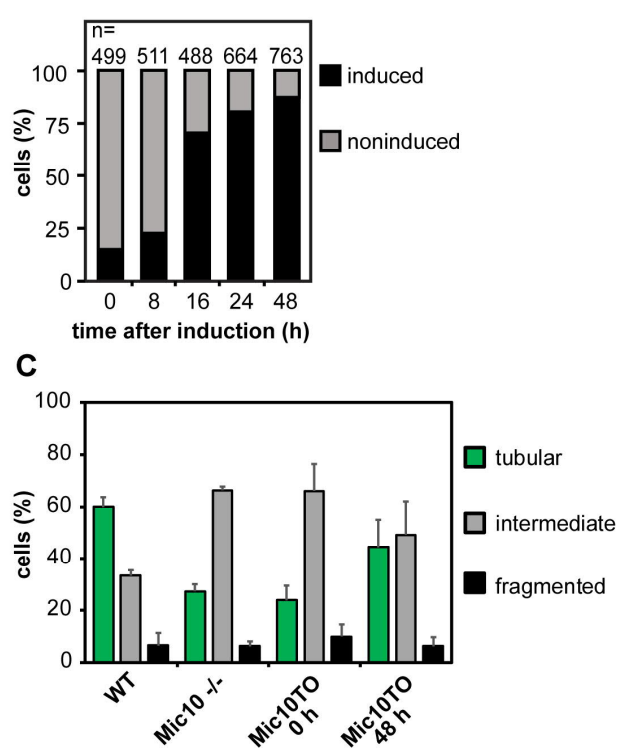

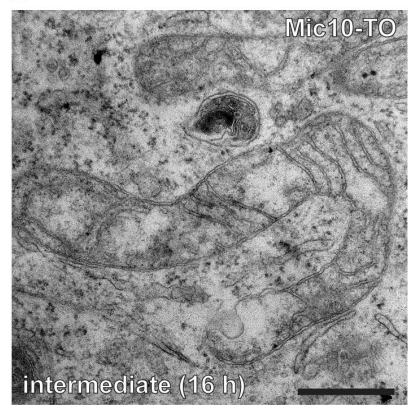

Appendix Fig S5. Re-expression of Mic10-FLAG in Mic10-TO cells rescues the morphology of the mitochondrial network. (A) MICOS-subunit expression levels in WT, Mic10-KO and induced or noninduced Mic 10TO cells. FLAG antibodies were used to pull-down Mic10-FLAG and interacting proteins from cell lysates. Mic60antibodies were used to pull-down Mic60 and interacting proteins from cell lysates. Samples were analyzed by Western blotting. (B) Quantification of induced Mic10-TO cells. Cells were induced with doxycycline for the indicated time periods and analyzed. Cells that expressed visible amounts of Mic10 were counted as induced. n: Number of cells analyzed. (C) Quantification of mitochondrial network remodeling upon Mic10-FLAG re-expression in Mic10TO cells. For the analysis, cells were immunolabeled for TOM20, imaged by confocal fluorescence microscopy, and manually annotated. Average and SD of three independent biological repeats are shown. For each repeat, at least 170 cells per sample were analyzed. (D) Remodeling of the CMs. Induced Mic10-TO cells were analyzed by TEM. The image shows a representative mitochondrion with an intermediate cristae architecture after $16 \mathrm{~h}$ of induction. Scale bar: $1 \mu \mathrm{m}$. 

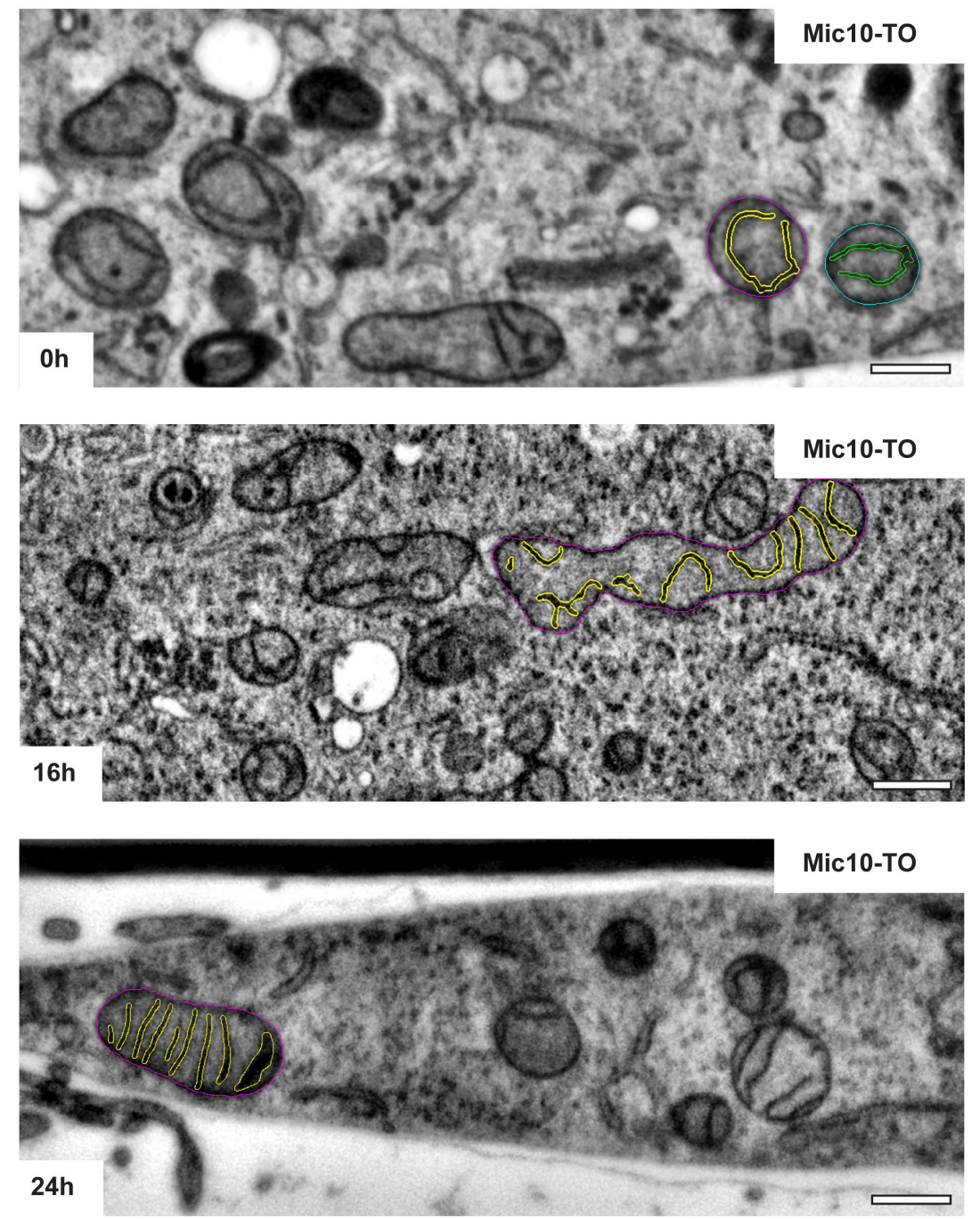

Appendix Fig S6. Holo-MICOS complex assembly induces remodeling of the inner membrane. FIB-SEM of Mic10-TO cells after induction of re-expression of Mic10-FLAG. Cells were induced for 0,16 and $24 \mathrm{~h}$ and analyzed by FIB-SEM. Shown are single sections from representative FIB-SEM stacks. The mitochondria that were reconstructed for Fig 7A are highlighted by colored lines. Scale bars: $1 \mu \mathrm{m}$. 
Appendix Table S1. CRISPR/Cas9 mediated disruption of MICOS genes in HeLa cells. For the generation of KO-cell lines, gRNAs against the shown target sequences were used. Cell lines were verified by sequencing as well as by Western blotting. For sequencing, PCR products were sub-cloned. Approximately 20 sub-clones were sequenced for each cell line. The table indicates the frequency of specific indels found by sequencing of the subclones for each cell line. No WT alleles could be identified for any of the MICOS mutant cell lines.

\begin{tabular}{|c|c|c|c|c|c|}
\hline $\begin{array}{l}\text { MICOS } \\
\text { Subunit }\end{array}$ & $\begin{array}{l}\text { Target } \\
\text { Exon }\end{array}$ & Allele & Mutation & Sequence $\left(5^{\prime}-3^{\prime}\right)$ & Frequency \\
\hline \multirow{3}{*}{ Mic10 } & \multirow{3}{*}{ Exon 1} & $\mathrm{Wt}$ & & CGGAGCTCGGCAGGA & $0 \%$ \\
\hline & & Mut1 & $\begin{array}{l}\text { Nonsense: Insertion leading to a } \\
\text { frameshift and an early stop } \\
\text { codon in the first exon }\end{array}$ & CGGAGCTCTCGGCAGGA & $75 \%$ \\
\hline & & Mut2 & $\begin{array}{l}\text { Nonsense: Deletion leading to a } \\
\text { frameshift and an early stop } \\
\text { codon in the first exon }\end{array}$ & CGGAGCT_GGCAGGA & $25 \%$ \\
\hline \multirow{3}{*}{ Mic13 } & \multirow{3}{*}{ Exon 3} & $\mathrm{Wt}$ & & CGCCGTCTACCTGGT & $0 \%$ \\
\hline & & Mut1 & $\begin{array}{l}\text { Nonsense: Insertion leading to a } \\
\text { frameshift and an early stop } \\
\text { codon in the fourth exon }\end{array}$ & CGCCGTCT $\underline{T A C C T G G T ~}$ & $72 \%$ \\
\hline & & Mut2 & $\begin{array}{l}\text { Nonsense: Deletion leading to a } \\
\text { frameshift and an early stop } \\
\text { codon in the fourth exon }\end{array}$ & CGCCGTCT _ _CTGGT & $28 \%$ \\
\hline \multirow{3}{*}{ Mic19 } & \multirow{3}{*}{ Exon 1} & $\mathrm{Wt}$ & & GAACATCACCGTGGT & $0 \%$ \\
\hline & & Mut1 & $\begin{array}{l}\text { Nonsense: Deletion leading to a } \\
\text { frameshift and an early stop } \\
\text { codon in the second exon }\end{array}$ & GAACATCA_CGTGGT & $72 \%$ \\
\hline & & Mut2 & $\begin{array}{l}\text { Nonsense: Insertion leading to a } \\
\text { frameshift and an early stop } \\
\text { codon in the second exon }\end{array}$ & GAACATCA $\underline{A} C C G T G G T$ & $28 \%$ \\
\hline \multirow{3}{*}{ Mic25 } & \multirow{3}{*}{ Exon2 } & $\mathrm{Wt}$ & & CTTTGGCCTTCAAGA & $0 \%$ \\
\hline & & Mut1 & $\begin{array}{l}\text { Nonsense: Deletion leading to a } \\
\text { frameshift and an early stop } \\
\text { codon in the second exon }\end{array}$ & CTTTGGCC $\ldots$ AGA & $68 \%$ \\
\hline & & Mut2 & $\begin{array}{l}\text { Nonsense: Deletion leading to a } \\
\text { frameshift and an early stop } \\
\text { codon in the fourth exon }\end{array}$ & CTTTGGCCT $\ldots$ AAGA & $32 \%$ \\
\hline \multirow{3}{*}{ Mic26 } & \multirow{3}{*}{ Exon4 } & $\mathrm{Wt}$ & & TCAGTTCCTGAGGGT & $0 \%$ \\
\hline & & Mut1 & $\begin{array}{l}\text { Nonsense: Insertion leading to a } \\
\text { frameshift and an early stop } \\
\text { codon in the fourth exon }\end{array}$ & TCAGTTCCㅡTGAGGGT & $79 \%$ \\
\hline & & Mut2 & $\begin{array}{l}\text { Nonsense: Deletion leading to a } \\
\text { frameshift and an early stop } \\
\text { codon in the fifth exon }\end{array}$ & TCAGTTCC $=$ GAGGGT & $21 \%$ \\
\hline \multirow[b]{2}{*}{ Mic27 } & \multirow[b]{2}{*}{ Exon3 } & $\mathrm{Wt}$ & & GGTTGTTACATTGGC & $0 \%$ \\
\hline & & Mut1 & $\begin{array}{l}\text { Nonsense: Deletion leading to a } \\
\text { frameshift and an early stop } \\
\text { codon in the fifth exon }\end{array}$ & GGTTGTTA _ _TTGGC & $100 \%$ \\
\hline \multirow{3}{*}{ Mic60 } & \multirow{3}{*}{ Exon 1} & $\mathrm{Wt}$ & & GGGCCTGTCAGTTAT & $0 \%$ \\
\hline & & Mut1 & $\begin{array}{l}\text { Nonsense: Deletion leading to a } \\
\text { frameshift and an early stop } \\
\text { codon in the first exon }\end{array}$ & GGGCCTGT _. _ TAT & $55 \%$ \\
\hline & & Mut2 & $\begin{array}{l}\text { Nonsense: A Part of the first } \\
\text { Exon is missing, together with a } \\
\text { huge insertion leading to an early } \\
\text { stop codon in the first exon }\end{array}$ & $\ldots . .$. & $45 \%$ \\
\hline
\end{tabular}




\section{Chapter 5 - Discussion and Outlook}

\subsection{Short Summary}

The first part of this thesis describes the development of a new labeling approach that enables time-lapse live-cell STED nanoscopy of the mitochondrial inner membrane. Live-cell recordings allowed for the analysis of dynamic movements of cristae and MICOS clusters on a timescale of seconds, thereby opening the door for the investigation of cristae architecture and the dynamic movement of inner membrane proteins in the mitochondria.

Using the developed live-cell fluorescence nanoscopy together with electron microscopy and biochemical analysis, the second part of this work focuses on the roles of MICOS, the dynamin-like GTPase OPA1, and the dimeric $\mathrm{F}_{1} \mathrm{~F}_{\mathrm{O}}$-ATP synthase in cristae formation and maintenance. The presented data suggest an intricate interplay of these factors in the shaping of the inner membrane. The Mic60subcomplex exhibits a coordinated distribution in the inner membrane that controls the positioning of crista junctions in cooperation with the Mic10-subcomplex, OPA1, and the dimeric $\mathrm{F}_{1} \mathrm{~F}_{\mathrm{O}}$-ATP synthase. Assembly of the holo-MICOS complex triggers extensive remodeling of the inner membrane to facilitate the formation of lamellar cristae by the segmentation of larger cristae precursors and by the formation of secondary crista junctions. The formation of the lamellar cristae is largely independent from mitochondrial tubule fusion and fission in mammals, suggesting surprising differences in cristae formation between lower and higher eukaryotes. Finally, the data presented in this thesis allowed for the development of a new model of cristae formation in mammals.

\subsection{Nanoscopy Reveals Inner Membrane Dynamics}

Early studies analyzing mitochondria by electron tomography predicted that cristae are pleomorphic structures that can dynamically change their appearance (Mannella, 2006). This idea could not be tested at that time because visualization of the cristae architecture relied exclusively on electron microscopy of fixed specimens. Live-cell compatible super-resolution microscopy or nanoscopy techniques were still in early development. Since then, these techniques have been dramatically improved and have become basic tools in cell biology (Jakobs et al., 2020; Sahl et al., 2017). Chapter 2 of this work describes the development of a new labeling strategy for the mitochondrial inner membrane that allowed for analysis of individual cristae in living cells by STED nanoscopy. Corroborating the predictions from early studies, live-cell recordings at approximately $50 \mathrm{~nm}$ resolution indeed revealed motile cristae constantly altering their appearance on a timescale of seconds. Interestingly, a dynamic behavior of the cristae was independently verified by several recent studies that used different cell lines, labeling approaches and imaging techniques, suggesting that a dynamic behavior of cristae is a common phenomenon (Huang et al., 2018; Kondadi et al., 2019; Wang et al., 2019). The use of the SNAP-tag and a silicon rhodamine dye (Juillerat et al., 2003; Lukinavičius et al., 2013) facilitated robust imaging of lamellar cristae, but also allowed aberrant cristae to be followed for the first time in MICOS-deficient cells. Moreover, a similar labeling strategy 
revealed the dynamic behavior of Mic10 protein clusters inside the inner membrane. These results clearly demonstrate that fast live-cell fluorescence nanoscopy will benefit the investigation of inner membrane dynamics in the future (Jakobs et al., 2020). The fact that cristae are densely packed inside the mitochondrial tubules complicates their visualization and the analysis of their dynamics. Extended-resolution microscopy techniques like structured illumination microscopy (SIM) (Gustafsson, 2000) or image scanning microscopy (ISM or Airyscan microscopy) (Müller and Enderlein, 2010) improve the spatial resolution of conventional fluorescence microscopes approximately by a factor of two, enabling high-speed imaging of the inner membrane (Huang et al., 2018). Nevertheless, these techniques are diffraction-limited and thus are restricted to a spatial resolution of about 100-120 nm. In most cell types, the robust visualization of individual cristae requires spatial resolutions which are only feasible by diffraction-unlimited techniques like STED nanoscopy (Hell and Wichmann, 1994), reversible saturable optical linear fluorescence transitions (RESOLFT) nanoscopy (Grotjohann et al., 2011; Hell, 2005) or nonlinlear structured illumination microscopy (NLSIM) (Gustafsson, 2005). These techniques generally require specific fluorescent labels and apply higher light doses onto the sample than diffraction-limited microscopy techniques. This intensifies the need for labeling strategies that reduce photobleaching and phototoxic effects (Jakobs et al., 2020; Kilian et al., 2018; Sahl et al., 2017). Labeling of the inner membrane using the SNAP-tag and a silicone rhodamine dye enabled high labeling densities and minimal phototoxic effects, but the number of recordable frames was restricted to about 20 images due to photobleaching. Labeling with the SNAP-tag, however, delivers the flexibility to use different, possibly more photostable dyes in the future. Reduced photobleaching would allow the mitochondrial inner membrane to be recorded over extended periods. So far, all studies reporting on live-cell nanoscopy of the inner membrane, including the one presented in this thesis, presented single-color recordings (Kondadi et al., 2020; Wang et al., 2019). As several different proteins control cristae formation, future analysis would clearly benefit from multicolor live-cell nanoscopy of the inner membrane. The stable cell line developed in this thesis will be a valuable tool to develop such a multi-color live-cell nanoscopy approach as it allows co-expression of proteins labeled with the bioorthogonal self-labeling Halo-Tag and a flexible combination of various nanoscopy-compatible dyes.

\subsection{Cristae Formation in Mitochondria}

\subsubsection{MICOS controls inner membrane remodeling}

By depleting all known MICOS subunits individually in the same human cell line, this work allowed for the dissection of the functions of the two MICOS subcomplexes in mammals. As in yeast, depletion of Mic10 destabilizes the human Mic10-subcomplex whereas the Mic60subcomplex remains intact (Guarani et al., 2015; Malsburg et al., 2011). Loss of the Mic10subcomplex leads to the formation of aberrant, tube-like cristae that line the interior of the mitochondrial tubules. However, unlike in yeast, these Mic10-deficient mitochondria still 
exhibit a substantial number of tubular, slightly enlarged crista junctions connecting the tubelike cristae with the inner boundary membrane (Chapter 4, Fig 3). These crista junctions, which became obvious in electron tomography recordings of thicker mitochondrial sections, exhibit an irregular distribution that complicates their detection on ultrathin sections by transmission electron microscopy. This observation might explain the fact that previous studies, relying on standard transmission EM, reported a virtual lack of crista junctions in Mic10- and Mic13depleted mammalian cells (Anand et al., 2016; Kondadi et al., 2020).

Depletion of Mic60 in human cells causes the loss of both MICOS subcomplexes, suggesting that the Mic60-subcomplex controls cristae formation upstream of the Mic10-subcomplex in mammals. This observation demonstrates a striking difference in the regulation of the MICOS complexes from higher and lower eukaryotes, since Mic60 depletion in yeast causes only destabilization of the Mic60-subcomplex (Malsburg et al., 2011). As crista junctions are virtually absent in human Mic60 knockout cells, but not in human Mic10 knockout cells, the data allow, for the first time, the disentanglement of cristae formation and crista junction formation. Electron microscopy recordings substantiate that, in humans, the Mic60-subcomplex is crucial for crista junction formation, whereas the Mic10-subcomplex modulates shaping of the cristae (Chapter 4, Fig 3). Tetracycline-inducible Mic10-TO and Mic60-TO cell lines produced in this work allowed for analysis of the restoration of the cristae and crista junctions upon re-expression of either the Mic10-subcomplex (Mic10-TO cells) or the entire MICOS complex (Mic60-TO cells). Mic10-TO cells thereby allowed for analysis of the consequences of a re-constitution of the holo-MICOS complex from its Mic60- and Mic10-subcomplexes. Rescue of Mic10 levels induced folding and fission of larger, unstructured cristae into multiple highly ordered lamellar cristae and a redistribution of the crista junctions (Chapter 4, Fig 7). The development of intermediately shaped cristae suggests that MICOS assembly induces remodeling of preexisting cristae membrane rather than the formation of new cristae. These observations establish the idea that assembly of the holo-MICOS complex acts as a switch, which controls inner membrane remodeling and cristae shaping. Analysis of Mic60-TO cells further illustrated that Mic60-subcomplexes enable the formation of secondary crista junctions by fusion of pre-existing cristae with the inner boundary membrane (Chapter 4, Fig 5).

\subsubsection{OPA1 stabilizes tubular crista junctions}

This study demonstrated that OPA1 depletion leads to a severe reduction in the number of crista junctions in Mic10 knockout cells. This finding suggests that OPA1 is necessary for the stabilization of crista junctions in the absence of a fully assembled MICOS complex and it indicates that OPA1 might support the MICOS-dependent attachment of cristae to the inner boundary membrane. Indeed, crosslinking and complex-immunoprecipitation experiments have suggested a direct interaction of OPA1 with the Mic60-subcomplex via Mic19, and thereby coordinated functioning of MICOS and OPA1 (Darshi et al., 2010; Glytsou et al., 2016). Moreover, a recent study proposed that assemblies of Mgm1, the OPA1 homolog from 
yeast, mediate inner membrane fusion by the formation of small tips in the inner membrane, which might also be essential to support MICOS-dependent reattachment of cristae (Yan et al., 2020).

OPA1-dependent stabilization of tubular crista junctions, as observed in Mic10 knockout cells, is in line with previous data demonstrating that OPA1 controls the crista junction diameter in apoptosis (Frezza et al., 2006). Recent cryo-EM data of Mgm1 from Chaetomium thermophilum could provide a conclusive explanation for this stabilization effect. Purified Mgm1 bent artificial membranes into tubular structures by forming a helical filament on the outer leaflet or extended patches on the inner leaflet of lipid vesicles. Faelber and colleagues proposed that Mgm1 forms such helical arrangements inside of crista junctions to shape the membrane and to constrict the crista junctions in a GTP-dependent manner (Faelber et al., 2019). Future studies will need to investigate whether OPA1 can also form these coordinated assemblies since the sequence homology of the two proteins is quite low. However, biochemical data support the idea, as the function of OPA1 strongly depends on its ability to oligomerize (Cipolat et al., 2004; Frezza et al., 2006; Patten et al., 2014).

\subsubsection{MICOS exhibits a coordinated distribution}

The results presented in this thesis demonstrate that Mic60's role in crista junction formation and its function in contact site formation are strongly connected to each other. In yeast cells, human dermal fibroblasts, and in Mic10 knockout HeLa cells, distinct Mic60 clusters, reflecting the crista junctions, were found to form opposite Mic60 distribution bands that run along the mitochondrial tubules (Chapter 3, Figure 1 and 2). Several findings argue against the notion that this two-sided Mic60 arrangement is just a consequence of the cristae architecture, instead indicating that it is an intrinsic feature of mitochondria. In human dermal fibroblasts, Mic60 clusters in one distribution band correlate with the ones on the other side of the mitochondrial tubule, although the shape of the cristae is quite irregular. In yeast mitochondria, the small cristae are rather short and thus cannot connect the opposite Mic60 clusters. Moreover, although Mic10 depletion disturbs the cristae architecture in yeast mitochondria, it barely influences Mic60's positioning. Together, these findings strongly suggest that the opposite arrangement of Mic60 clusters is largely independent from the cristae. In fibroblasts and yeast cells, the opposite Mic60 distribution bands frequently adopt a twisted arrangement that coincides with a propeller-like arrangement of the cristae in yeast mitochondria, as visualized by FIB-SEM. The opposite positioning of Mic60 clusters and the helical arrangement of the Mic60 distribution bands indicate that the crista junctions can be physically coupled along and across the mitochondrial tubules. As this coupling cannot be attributed to the cristae, these findings suggest that proteins within the mitochondrial membranes control this intriguing arrangement. Most likely, the arrangement of Mic60 is supported by the mitochondrial intermembrane space bridging (MIB) complex that links Mic60 to the outer membrane (Ding et al., 2015; Körner et al., 2012; Ott et al., 2012; Tang et al., 2019; Zerbes et 
al., 2012). The MIB complex is regulated by Mic19, which connects Mic60 with the SAM50 subunit of the SAM complex. Disruption of the Mic60-Mic19-SAM50 axis disturbs the cristae architecture and disrupts crista junctions, suggesting that the MIB complex is involved in crista junction positioning (Ding et al., 2015; Ott et al., 2012; Tang et al., 2019). The aberrant distribution of Mic60 in Mic19 knockout cells and the observation that overexpression of Mic10 in Mic19 knockout cells induced a restoration of Mic60 levels, but failed to induce a proper Mic60 distribution, further validate the importance of Mic19 for MICOS positioning in the inner membrane.

\subsubsection{Mic10 regulates the width of the Mic60 distribution bands}

Mitochondria from HeLa cells exhibit a rather large diameter $(400-800 \mathrm{~nm})$ and feature welldeveloped lamellar cristae. Unlike the small cristae in yeast mitochondria, each of the large lamellar cristae in HeLa cells features multiple adjacent crista junctions, as visualized by electron tomography. Therefore, the crista junctions virtually encircle the mitochondrial tubules, leading to a stripe-like distribution of Mic60 and Mic10 on STED recordings of wild type HeLa cells. Remarkably, opposite Mic60 distribution bands were observed in this cell line upon the depletion of the Mic10-subcomplex, although the aberrant cristae in Mic10-deficient cells formed symmetrical tube-like structures that lined the interior of the organelle. This demonstrates that also in HeLa cells opposite Mic60 distribution bands exist at low expression levels of the Mic10-subcomplex. Along the distribution bands, Mic60-subcomplexes facilitate the formation of crista junctions, although the overall number is reduced in the absence of Mic10. Consequently, electron tomography of Mic10 knockout cells revealed an altered distribution of the crista junctions compared to the stripe-like arrangements of crista junctions in wild type cells. Expression of Mic10 in Mic10-deficient cells stabilized the components of the Mic10-subcomplex and recruited them to the Mic60-subcomplexes. The formation of the holo-MICOS complex induced the spreading of MICOS clusters over the mitochondrial tubules and the opposite distribution bands were no longer visible on STED recordings (Chapter 4, Fig 6). This redistribution of MICOS clusters promotes the idea that the Mic10-subcomplex controls the width of the Mic60-distributions bands and facilitates efficient movement of MICOS clusters and crista junctions around the mitochondrial tubules. Knockdown experiments illustrate that the dimeric $\mathrm{F}_{1} \mathrm{~F}_{\mathrm{O}}$-ATP synthase, which interacts directly with the Mic10-subcomplex (Eydt et al., 2017; Rampelt et al., 2017b), supports this even distribution of MICOS clusters and crista junctions around the mitochondrial tubules. Thereby, fhe findings described in this study strongly suggest that mitochondria exhibit an intricate mechanism that allows them to adapt the number and the distribution of crista junctions to the size of the cristae. As MICOS supports attachment of cristae to the inner membrane, widening of the Mic60distribution bands possibly increases the number of potential attachment sites and thus supports the formation and stability of the large lamellar cristae. 


\subsubsection{Interplay of MICOS and OPA1 controls MICOS assemblies}

In addition to the interplay between the two MICOS-subcomplexes, proper distribution of MICOS clusters around the mitochondria requires interplay of MICOS with the dynamin-like GTPase OPA1. This study revealed that depletion of OPA1 causes the formation of large, continuous, rib-like Mic60 assemblies that seem to encircle the mitochondria. Intriguingly, these assemblies form in a Mic10-dependent manner as Mic10 knockout cells depleted of OPA1 exhibited only randomly distributed Mic60 clusters (Figure 17).

Although conflicting conclusions about the precise function of OPA1 have been made, virtually all studies reported on a disturbed cristae architecture in OPA1-deficient cells (Barrera et al., 2016; Frezza et al., 2006; Glytsou et al., 2016; MacVicar and Langer, 2016). In some studies, knockout of OPA1 strongly reduced the overall number of cristae (Glytsou et al., 2016; Patten et al., 2014; Zhang et al., 2011) whereas in other studies a reduced number of crista junctions in OPA1 knockout cells was observed (Glytsou et al., 2016; Lee et al., 2017).

The knockdown experiments presented in this work lead to the conclusion, that efficient but transient depletion of OPA1 causes a rather mild cristae phenotype compared to knockdowns of Mic60 or Mic10. Different cristae phenotypes reported for knockout or knockdown cells might be a consequence of long-term OPA1 depletion in knockout cells. Stable loss of Mgm1 in yeast disturbs, for example, lipid homeostasis and it impairs genome maintenance (Kojima et al., 2019). It is likely that similar effects also afflict OPA1 knockout cells (Elachouri et al., 2010). Such secondary effects might promote more severe inner membrane phenotypes upon long-term depletion of OPA1. Short-term depletion of OPA1, however, does not inhibit the formation of lamellar cristae or of crista junctions in general. In fact, rescue of the Mic10 levels in OPA1-deficient Mic10 knockout cells still induced remodeling of the inner membrane into (disordered) lamellar cristae and the formation of elongated Mic60 assemblies as observed in OPA1-deficient wild type cells. The data presented in this study thereby contradict previous assumptions that OPA1 controls cristae formation upstream of Mic60 (Glytsou et al., 2016). On the contrary, the presented findings indicate that holo-MICOS assembly triggers lamellar cristae formation upstream of OPA1. Nevertheless, OPA1 is crucial to control the distribution and size of Mic60 assemblies during this process. The results therefore support that OPA1 is generally dispensable for lamellar cristae formation but cooperates with Mic10 to stabilize crista junctions and to facilitate the formation of highly ordered cristae.

Electron tomography and STED nanoscopy data presented in Chapter 4 suggest that the interplay between the Mic10-subcomplex and OPA1 can influence the size and shape of crista junctions. The rib-like Mic60 assemblies in wild type cells devoid of OPA1 coincided with the appearance of extended slit-like crista junctions on electron tomography recordings (Chapter 4, Fig 8 and Fig 9). In contrast to this situation, Mic10 knockout cells exhibited only circular crista junctions (Chapter 4, Fig 3) that were stabilized by OPA1. In wild type cells, where both 
proteins are present, crista junctions had a circular or slightly elongated shape and were aligned in stripes perpendicular to the longitudinal axis of the mitochondrion.

Based on these observations, I suggest that the Mic10-subcomplexes promote not only transversal spreading of MICOS clusters around the mitochondrial tubules, but could also support transversal elongation of crista junctions. OPA1, in turn, by stabilizing tubular membrane structures could restricts the size of MICOS assemblies. Therefore, the interplay of MICOS and OPA1 could be part of an efficient mechanism to control the size and shape of crista junctions (Figure 17). However, verification of this idea will require further investigation by electron tomography of entire mitochondria to measure the dimensions of the crista junctions. The electron tomograms shown in this thesis cover only sections of about $200 \mathrm{~nm}$ along the z-axis. Still, the dimension and the distribution of the crista junctions generally matched the size and the distribution of Mic60 assemblies on STED recordings. The idea of Mic10-driven crista junction elongation is indeed supported by the formation of slit-like crista junctions upon Mic10 overexpression in yeast (Bohnert et al., 2015). In addition, another study in yeast demonstrated that inactivation of Mgm1-5, a heat-sensitive version of Mgm1, leads to the formation of septa junctions, slit-like crista junctions that encircle the entire mitochondrial tubule and divide the matrix into physically separated compartments (Harner et al., 2016). Although the authors of the respective study accounted the formation of these inner membrane septa for improper inner membrane fusion (Harner et al., 2016), the formation of these structures could also denote uncontrolled widening or fusion of crista junctions caused by Mgm1 inactivation in the presence of the Mic10.

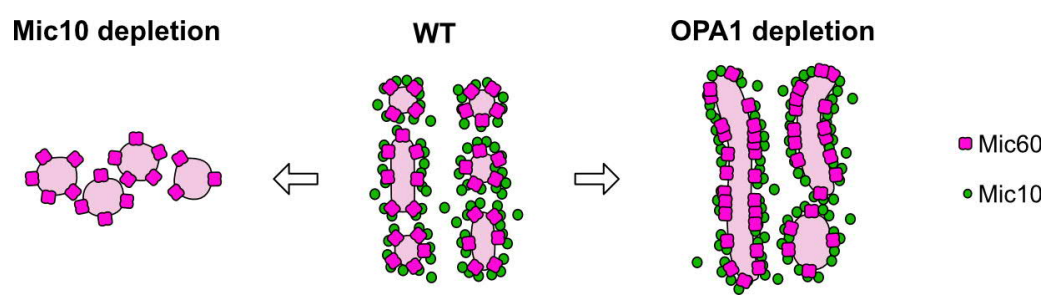

Figure 17: Model for the regulation of Mic60 assemblies. Schematic representation of the formation of Mic60 assemblies at crista junctions. In wild type cells (WT), Mic60 forms distinct clusters that are oriented in stripes perpendicular to the length axis of the mitochondrion. Loss of Mic10 leads to a redistribution of Mic60 clusters and crista junctions into two opposite distribution bands. In the absence of OPA1, Mic10 induces the formation of extended Mic60 assemblies.

\subsubsection{A new model of cristae formation}

Systematic depletion of the individual MICOS subunits, OPA1 and ATP5ME in HeLa cells demonstrated that mitochondria of cells lacking Mic60 exhibit the most severe phenotype among all analyzed inner membrane-shaping proteins. Although these cells lose virtually all crista junctions, they can still assemble OXPHOS complexes and form cristae membrane. This underlines that MICOS generally coordinates cristae formation but does not drive the 
biogenesis of the cristae membrane (Harner et al., 2014, 2016). In the absence of Mic10, the Mic60-subcomplex remains intact and enables the formation of crista junctions. Upon reexpression of Mic10 in Mic10-depleted cells, assembly of the holo-MICOS complex triggers the folding and fission of the aberrant tube-like cristae into highly ordered lamellar cristae.

The inner membrane remodeling mechanisms observed during this repair process provide insights into the mechanisms, which most likely also control de novo cristae formation. Recruitment of the Mic10-subcomplex subunits by the Mic60-subcomplex demonstrates that the Mic60-subcomplex acts upstream of the Mic10-subcomplex in cristae formation. Most likely, the tube-like cristae structures in Mic10-deficient cells therefore represent a trapped and massively enlarged intermediate of cristae formation that originates from impaired shaping of the cristae membrane. Based on this assumption, I propose a new model of cristae formation that considers the coordinated distribution of MICOS in the inner membrane, MICOSdependent membrane remodeling, and the interplay of MICOS with OPA1 and with the dimeric $\mathrm{F}_{1} \mathrm{~F}_{\mathrm{O}}$-ATP synthase (Figure 18).

This membrane-coordination model suggests that cristae biogenesis starts in a MICOSindependent way by proliferation of the inner membrane. The process is spatially coordinated both by the Mic60-subcomplexes and by OPA1, which collectively enable the formation of stable crista junctions. Fusion of the cristae membrane with the inner boundary membrane allows for the formation of additional, secondary crista junctions attaching the cristae membrane to the inner boundary membrane (Figure 18).

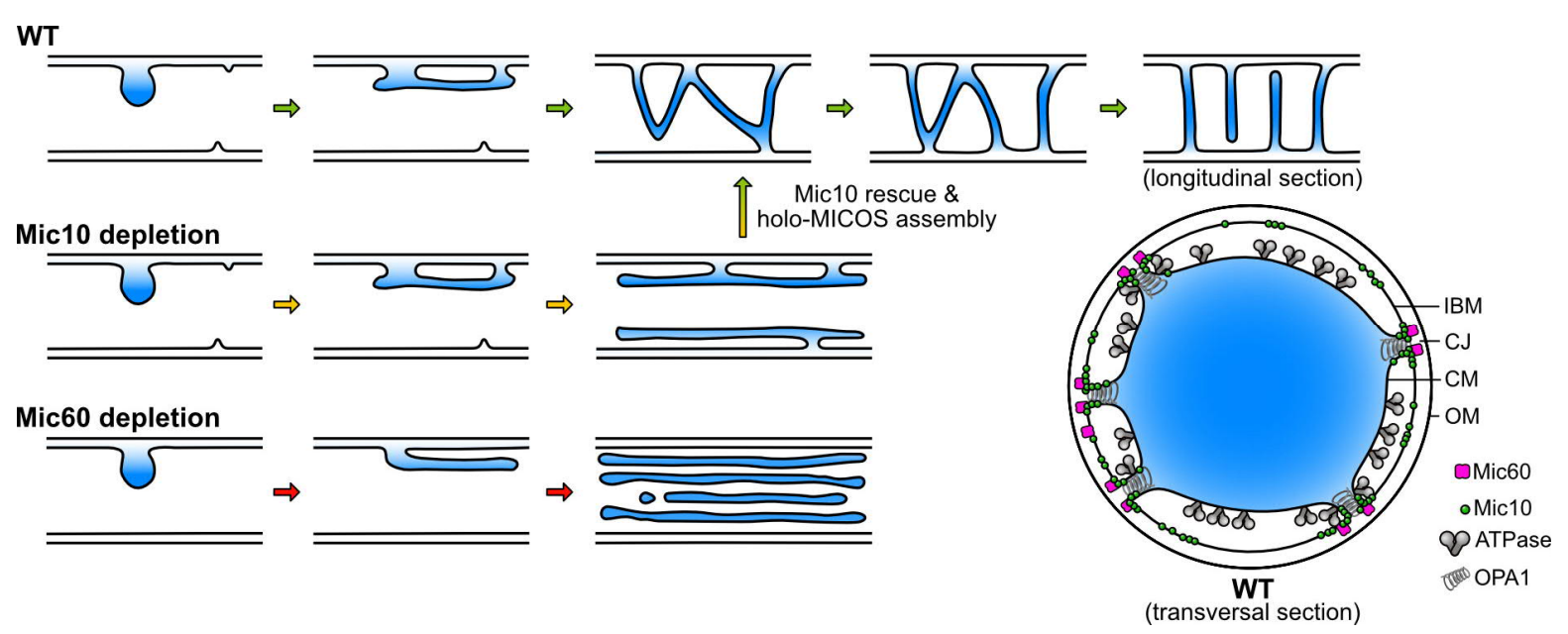

Figure 18: Model for lamellar cristae formation. Left: Model for the formation of the inner membrane structures in mitochondria of wild type (WT), Mic10 depleted and Mic60 depleted cells. Assembly of the holo-MICOS complex controls inner membrane remodeling. Lower right: Side view on a single lamellar crista of a WT cell. Supposed localization of membrane shaping proteins is shown.

Recruitment of the Mic10-subcomplex to the curved membranes at crista junctions, presumably supported by cardiolipin-binding of Mic27, leads to the formation of the holo-MICOS complex (Friedman et al., 2015; Rampelt et al., 2017a; Weber et al., 2013). Assembly of the holo- 
MICOS complex intensifies membrane curvature at crista junctions and controls the coordinated shaping of the cristae membrane. During this process, larger unstructured membranes can be separated into multiple lamellae that are stabilized by the formation of additional crista junctions and by rows of the dimeric $\mathrm{F}_{1} \mathrm{~F}_{\mathrm{O}}$-ATP synthase. The direct interaction of the Mic10-subcomplex with the dimeric $\mathrm{F}_{1} \mathrm{~F}_{\mathrm{O}}$-ATP synthase (Eydt et al., 2017; Rampelt et al., 2017b) might facilitate a structural connection between the crista junctions and the cristae membrane to promote establishment and organization of the cristae. Finally, intricate interplay between MICOS, the dimeric $\mathrm{F}_{1} \mathrm{~F}_{\mathrm{O}}$-ATP synthase, and OPA1 fine-tunes the optimal distribution of the crista junctions around the mitochondrial tubules as described above in more detail.

The membrane coordination model combines elements of the membrane invagination or balloon model with coordinated fusion and fission of the inner membrane (Figure 14) (Zick et al., 2008). In this model, fusion of the inner membrane supports the formation of secondary crista junctions whereas fission allows for the segmentation of membranes into individual cristae. Regarding the key players involved in cristae formation, the described model takes into consideration the central aspects that have been demonstrated by earlier studies and by the data presented in this thesis. The Mic60-dependent formation of crista junctions and the Mic10induced remodeling of the inner membrane are generally in line with the presented electron microscopy data and reports about the membrane-bending abilities of Mic10 and Mic60 (Barbot et al., 2015; Bohnert et al., 2015; Hessenberger et al., 2017; Tarasenko et al., 2017). The OPA1dependent formation of crista junctions in Mic10 knockout cells (Chapter 4) and the recent findings about the formation of Mgm1 assemblies inside lipid tubes (Faelber et al., 2019) are also covered by the membrane-coordination model. Moreover, the model considers the influence of the dimeric $\mathrm{F}_{1} \mathrm{~F}_{\mathrm{O}}$-ATP synthase on the Mic60 distribution and its influence on the inner membrane shape (Kühlbrandt, 2019). The two-step assembly of the holo-MICOS complex described by the membrane-coordination model is underscored by the data from Mic10-TO cells and was recently independently supported by the finding that dynamic remodeling of the inner membrane requires Mic13 which regulates Mic10 stability (Kondadi et al., 2020).

Finally, the proposed membrane-coordination model is in line with the reported direct interactions of all involved membrane-shaping proteins (Darshi et al., 2010; Eydt et al., 2017; Glytsou et al., 2016; Quintana-Cabrera et al., 2018; Rampelt et al., 2017b).

In contrast, several data presented in this work, question the relevance of some of the previously suggested models of cristae formation in human cells (Chapter 1.5). STED and TEM data presented in this work support the idea that the dimeric $\mathrm{F}_{1} \mathrm{~F}_{\mathrm{O}}$-ATP synthase is required to coordinate lamellar cristae (Blum et al., 2019; Davies et al., 2012; Kühlbrandt, 2019). However, these dimers are not sufficient to promote lamellar cristae formation in Mic10-deficient cells. Therefore, the findings do not support the idea, that oligomerization of the $\mathrm{F}_{1} \mathrm{~F}_{\mathrm{O}}$-ATP synthase is the primary factor determining the cristae shaping in HeLa cells (Davies et al., 2012). 
In yeast, experimental data provided evidence for the existence of two distinct pathways of cristae formation that regulate the biogenesis of tubular and lamellar cristae, respectively (Figure 16). According to this model of cristae formation, tubular cristae are formed by a MICOS-coordinated invagination of the inner membrane, whereas the formation of larger crista lamellae requires the remodeling of inner membrane septa by MICOS and Mgm1 (Harner et al., 2016). Reduction of the fusion-fission dynamics of mitochondrial tubules impairs the formation of such septa, which result from incomplete fusion of mitochondrial tubules. Fission incompetent $\Delta d n m l$ yeast cells therefore develop only tubular cristae. In human HeLa cells, however, transient depletion of crucial fusion or fission factors like MFN1, MFN2, and DRP1 did not abolish the formation of lamellar cristae. These findings demonstrate that lamellar cristae formation does not require fusion and fission of the mitochondrial tubules in humans. It is therefore inconceivable that the hemifusion or the fusion remnant model (Zick et al., 2008) describe the primary mechanisms of cristae formation in humans (Figure 15). Electron microscopy recordings generally did not support the existence of two distinct cristae shapes in HeLa cells, suggesting substantial differences in cristae formation between higher and lower eukaryotes. As mentioned above, the regulation of the MICOS-subcomplexes also exhibits surprising differences between yeast and humans. However, the described findings do not exclude the possibility that the conversion of a fusion-derived septum can also lead to the formation of lamellar cristae in humans (Harner et al., 2016; Kojima et al., 2019; Zick et al., 2008). The remodeling of a septum into a lamellar crista would require the segmentation of a septa junction into circular or slightly elongated crista junctions. This process would most likely depend on the same functioning of Mic60, OPA1 and Mic10 that is necessary to form and maintain crista junctions as suggested by the membrane-coordination model.

Different assumptions about the mechanism of crista formation might be a result of unexpected differences between mitochondria from yeast, flies and humans (Harner et al., 2016; Jiang et al., 2019; Kojima et al., 2019; Rabl et al., 2009). The membrane-coordination model developed in this work is based on data from human HeLa cells that exhibit exclusively lamellar cristae. It is conceivable that cells with a different cristae architecture use other mechanisms to shape and maintain their cristae. However, since a coordinated segmentation of unstructured cristae membrane into distinct cristae and dynamic inner membrane remodeling could lead to various cristae shapes, the proposed model might describe a general mechanism prevalent in all mitochondria.

\subsection{Synopsis and Outlook}

Since its discovery in 2011, MICOS has turned out to be one of the most important determinants of the mitochondrial membrane architecture. Remarkably, one of the studies that discovered MICOS suggested that it may form a filamentous skeleton inside the inner membrane (Hoppins et al., 2011). Despite significant effort, such a continuous structure has not been observed using 
super-resolution or electron microcopy (Jans et al., 2013; Modi et al., 2019). Nevertheless, MICOS certainly fulfils functions expected from a "mitoskeleton". It anchors, distributes and stabilizes the crista junctions and it controls the fold of the inner membrane. MICOS mediates the formation of contact sites between the inner and outer membrane and is physically connected to the machinery in the outer membrane that facilitates transport of the organelle (Malsburg et al., 2011; Xie et al., 2007). Moreover, MICOS is essential to maintain the tubular shape of mitochondria. On super-resolution microscopy images, MICOS features a coordinated clustered distribution, suggesting that other interacting membrane proteins physically couple the MICOS clusters. Based on the data shown in this thesis, it is therefore tempting to assume that there is typically not a rigid filamentous structure, but rather a more flexible and dynamic elaborate network of membrane proteins, which includes MICOS, that functions as a "mitoskeleton".

The findings described in this thesis provide detailed insight into the interplay of the most important known inner membrane-shaping proteins and allowed for the development of a new model of lamellar cristae formation in humans. It is likely that future advances in cryo-electron tomography and super-resolution microscopy will enable deeper understanding of the processes that drive cristae biogenesis and will lead to further optimizations of this model. Indeed, several open questions still exist. Due to the lack of a crystal structure or cryo-EM structure of MICOS, the geometry and precise composition of the complex is unknown. It is also unclear if the two MICOS subcomplexes are stably associated or if the formation of the holo-MICOS complex occurs in a transient manner. The recently developed MINFLUX nanoscopy, which localizes proteins with nanometer precision, will help to investigate such questions regarding the dynamics of proteins on the single crista junction level. Moreover, establishment of long-term muti-color live-cell nanoscopy could clarify how the interplay between OPA1 and the Mic10subcomplex controls the size of MICOS assemblies and their distribution within the inner membrane. As cristae formation involves proteins as well as membranes, it is also likely that correlative approaches, combining electron and super-resolution microscopy, will be a valuable tool to enhance our knowledge about cristae biogenesis. 


\section{References}

Abrisch, R.G., Gumbin, S.C., Wisniewski, B.T., Lackner, L.L., and Voeltz, G.K. (2020). Fission and fusion machineries converge at ER contact sites to regulate mitochondrial morphology. J Cell Biol 219.

Alam, T.I., Kanki, T., Muta, T., Ukaji, K., Abe, Y., Nakayama, H., Takio, K., Hamasaki, N., and Kang, D. (2003). Human mitochondrial DNA is packaged with TFAM. Nucleic Acids Res 31, 1640-1645.

Alberts, B., Johnson, A., Lewis, J., Morgan, D., Raff, M., Roberts, K., and Walter, P. (2017). Molecular Biology of the Cell. 813-888.

Alexander, C., Votruba, M., Pesch, U.E.A., Thiselton, D.L., Mayer, S., Moore, A., Rodriguez, M., Kellner, U., Leo-Kottler, B., Auburger, G., et al. (2000). OPA1, encoding a dynamin-related GTPase, is mutated in autosomal dominant optic atrophy linked to chromosome 3q28. Nat Genet 26, 211-215.

Alkhaja, A.K., Jans, D.C., Nikolov, M., Vukotic, M., Lytovchenko, O., Ludewig, F., Schliebs, W., Riedel, D., Urlaub, H., Jakobs, S., et al. (2011). MINOS1 is a conserved component of mitofilin complexes and required for mitochondrial function and cristae organization. Mol Biol Cell 23, 247-257.

Anand, R., Wai, T., Baker, M.J., Kladt, N., Schauss, A.C., Rugarli, E., and Langer, T. (2014). The i-AAA protease YME1L and OMA1 cleave OPA1 to balance mitochondrial fusion and fission. J Cell Biology 204, 919-929.

Anand, R., Strecker, V., Urbach, J., Wittig, I., and Reichert, A.S. (2016). Mic13 Is Essential for Formation of Crista Junctions in Mammalian Cells. Plos One 11, e0160258.

Anderson, S., Bankier, A.T., Barrell, B.G., Bruijn, M.H.L. de, Coulson, A.R., Drouin, J., Eperon, I.C., Nierlich, D.P., Roe, B.A., Sanger, F., et al. (1981). Sequence and organization of the human mitochondrial genome. Nature 290, 457-465.

Arco, A. del, and Satrústegui, J. (2005). New mitochondrial carriers: an overview. Cell Mol Life Sci 62, 2204-2227.

Ardail, D., Privat, J.P., Egret-Charlier, M., Levrat, C., Lerme, F., and Louisot, P. (1990). Mitochondrial contact sites. Lipid composition and dynamics. J Biological Chem 265, 18797-18802.

Arnold, I., Pfeiffer, K., Neupert, W., Stuart, R.A., and Schägger, H. (1998). Yeast mitochondrial F1F0ATP synthase exists as a dimer: identification of three dimer-specific subunits. Embo J 17, 7170-7178.

Ban, T., Heymann, J.A.W., Song, Z., Hinshaw, J.E., and Chan, D.C. (2010). OPA1 disease alleles causing dominant optic atrophy have defects in cardiolipin-stimulated GTP hydrolysis and membrane tubulation. Hum Mol Genet 19, 2113-2122.

Banerjee, S., and Chinthapalli, B. (2014). A proteomic screen with Drosophila Opa1-like identifies Hsc70-5/Mortalin as a regulator of mitochondrial morphology and cellular homeostasis. Int $\mathrm{J}$ Biochem Cell Biology 54, 36-48.

Barbot, M., Jans, D.C., Schulz, C., Denkert, N., Kroppen, B., Hoppert, M., Jakobs, S., and Meinecke, M. (2015). Mic10 Oligomerizes to Bend Mitochondrial Inner Membranes at Cristae Junctions. Cell Metab $21,756-763$.

Barrera, M., Koob, S., Dikov, D., Vogel, F., and Reichert, A.S. (2016). OPA1 functionally interacts with MIC60 but is dispensable for crista junction formation. Febs Lett 590, 3309-3322.

Baxter, R.V., Othmane, K.B., Rochelle, J.M., Stajich, J.E., Hulette, C., Dew-Knight, S., Hentati, F., Hamida, M.B., Bel, S., Stenger, J.E., et al. (2001). Ganglioside-induced differentiation-associated protein-1 is mutant in Charcot-Marie-Tooth disease type 4A/8q21. Nat Genet 30, 21-22. 
Beltrán-Heredia, E., Tsai, F.-C., Salinas-Almaguer, S., Cao, F.J., Bassereau, P., and Monroy, F. (2019). Membrane curvature induces cardiolipin sorting. Commun Biology 2, 225.

Benda, C. (1898). Ueber die Spermatogenese der Vertebraten und höherer Evertebraten, II. Theil: Die Histiogenese der Spermien. Arch. Anat. Physiol. 73, 393-398.

Benz, R. (1994). Permeation of hydrophilic solutes through mitochondrial outer membranes: review on mitochondrial porins. Biochimica Et Biophysica Acta Bba - Rev Biomembr 1197, 167-196.

Bereiter-Hahn, J., and Vöth, M. (1994). Dynamics of mitochondria in living cells: Shape changes, dislocations, fusion, and fission of mitochondria. Microsc Res Techniq 27, 198-219.

Berg, J.M., Tymoczko, J.L., Gatto, G.J., and Stryer, L. (2017). Stryer Biochemie. 581-611.

Betzig, E., Patterson, G.H., Sougrat, R., Lindwasser, O.W., Olenych, S., Bonifacino, J.S., Davidson, M.W., Lippincott-Schwartz, J., and Hess, H.F. (2006). Imaging Intracellular Fluorescent Proteins at Nanometer Resolution. Science 313, 1642-1645.

Blum, T.B., Hahn, A., Meier, T., Davies, K.M., and Kühlbrandt, W. (2019). Dimers of mitochondrial ATP synthase induce membrane curvature and self-assemble into rows. Proc National Acad Sci 116, 42504255.

Bohnert, M., Wenz, L.-S., Zerbes, R.M., Horvath, S.E., Stroud, D.A., Malsburg, K. von der, Müller, J.M., Oeljeklaus, S., Perschil, I., Warscheid, B., et al. (2012). Role of mitochondrial inner membrane organizing system in protein biogenesis of the mitochondrial outer membrane. Mol Biol Cell 23, 39483956.

Bohnert, M., Zerbes, R.M., Davies, K.M., Mühleip, A.W., Rampelt, H., Horvath, S.E., Boenke, T., Kram, A., Perschil, I., Veenhuis, M., et al. (2015). Central role of Mic10 in the mitochondrial contact site and cristae organizing system. Cell Metab 21, 747-755.

Bolliger, L., Junne, T., Schatz, G., and Lithgow, T. (1995). Acidic receptor domains on both sides of the outer membrane mediate translocation of precursor proteins into yeast mitochondria. Embo J 14, 63186326.

Bornhövd, C., Vogel, F., Neupert, W., and Reichert, A.S. (2006). Mitochondrial Membrane Potential Is Dependent on the Oligomeric State of F 1 F 0 -ATP Synthase Supracomplexes. J Biol Chem 281, 13990-13998.

Bottanelli, F., Kromann, E.B., Allgeyer, E.S., Erdmann, R.S., Baguley, S.W., Sirinakis, G., Schepartz, A., Baddeley, D., Toomre, D.K., Rothman, J.E., et al. (2016). Two-colour live-cell nanoscale imaging of intracellular targets. Nat Commun 7, 10778.

Brickley, K., and Stephenson, F.A. (2011). Trafficking kinesin protein (TRAK)-mediated transport of mitochondria in axons of hippocampal neurons. J Biological Chem 286, 18079-18092.

Brito, O.M. de, and Scorrano, L. (2008). Mitofusin 2 tethers endoplasmic reticulum to mitochondria. Nature 456, 605-610.

Callegari, S., Cruz-Zaragoza, L.D., and Rehling, P. (2020). From TOM to the TIM23 complex - handing over of a precursor. Biol Chem 0.

Chacinska, A., Pfannschmidt, S., Wiedemann, N., Kozjak, V., Szklarz, L.K.S., Schulze-Specking, A., Truscott, K.N., Guiard, B., Meisinger, C., and Pfanner, N. (2004). Essential role of Mia40 in import and assembly of mitochondrial intermembrane space proteins. Embo J 23, 3735-3746.

Chakrabarti, R., Ji, W.-K., Stan, R.V., Sanz, J. de J., Ryan, T.A., and Higgs, H.N. (2017). INF2-mediated actin polymerization at the ER stimulates mitochondrial calcium uptake, inner membrane constriction, and division. J Cell Biology 217, 251-268. 
Cipolat, S., Brito, O.M. de, Zilio, B.D., and Scorrano, L. (2004). OPA1 requires mitofusin 1 to promote mitochondrial fusion. Proc National Acad Sci 101, 15927-15932.

Cogliati, S., Enriquez, J.A., and Scorrano, L. (2016). Mitochondrial Cristae: Where Beauty Meets Functionality. Trends Biochem Sci 41, 261-273.

Daems, W.T., and Wisse, E. (1966). Shape and attachment of the cristae mitochondriales in mouse hepatic cell mitochondria. J Ultra Mol Struct R 16, 123-140.

Darshi, M., Mendiola, V.L., Mackey, M.R., Murphy, A.N., Koller, A., Perkins, G.A., Ellisman, M.H., and Taylor, S.S. (2010). ChChd3, an inner mitochondrial membrane protein, is essential for maintaining crista integrity and mitochondrial function. J Biological Chem 286, 2918-2932.

Daum, B., Walter, A., Horst, A., Osiewacz, H.D., and Kühlbrandt, W. (2013). Age-dependent dissociation of ATP synthase dimers and loss of inner-membrane cristae in mitochondria. P Natl Acad Sci Usa 110, 15301-15306.

Davies, K.M., Strauss, M., Daum, B., Kief, J.H., Osiewacz, H.D., Rycovska, A., Zickermann, V., and Kühlbrandt, W. (2011). Macromolecular organization of ATP synthase and complex I in whole mitochondria. P Natl Acad Sci Usa 108, 14121-14126.

Davies, K.M., Anselmi, C., Wittig, I., Faraldo-Gómez, J.D., and Kühlbrandt, W. (2012). Structure of the yeast F1Fo-ATP synthase dimer and its role in shaping the mitochondrial cristae. P Natl Acad Sci Usa 109, 13602-13607.

Davies, K.M., Daum, B., Gold, V.A.M., Mühleip, A.W., Brandt, T., AU - Blum, T.B., AU - Mills, D.J., and AU - Kühlbrandt, W. (2014). Visualization of ATP Synthase Dimers in Mitochondria by Electron Cryotomography. JoVE e51228.

Davies, K.M., Blum, T.B., and Kühlbrandt, W. (2018). Conserved in situ arrangement of complex I and III 2 in mitochondrial respiratory chain supercomplexes of mammals, yeast, and plants. Proc National Acad Sci 115, 3024-3029.

Delettre, C., Lenaers, G., Griffoin, J.-M., Gigarel, N., Lorenzo, C., Belenguer, P., Pelloquin, L., Grosgeorge, J., Turc-Carel, C., Perret, E., et al. (2000). Nuclear gene OPA1, encoding a mitochondrial dynamin-related protein, is mutated in dominant optic atrophy. Nat Genet 26, 207-210.

Detmer, S.A., and Chan, D.C. (2007). Functions and dysfunctions of mitochondrial dynamics. Nat Rev Mol Cell Bio 8, 870-879.

DeVay, R.M., Dominguez-Ramirez, L., Lackner, L.L., Hoppins, S., Stahlberg, H., and Nunnari, J. (2009). Coassembly of Mgm1 isoforms requires cardiolipin and mediates mitochondrial inner membrane fusion. J Cell Biology 186, 793-803.

Ding, C., Wu, Z., Huang, L., Wang, Y., Xue, J., Chen, S., Deng, Z., Wang, L., Song, Z., and Chen, S. (2015). Mitofilin and CHCHD6 physically interact with Sam50 to sustain cristae structure. Sci Rep-Uk 5, 16064.

Dotto, V.D., Fogazza, M., Carelli, V., Rugolo, M., and Zanna, C. (2018). Eight human OPA1 isoforms, long and short: What are they for? Biochim Biophys Acta 1859, 263-269.

Dudkina, N.V., Heinemeyer, J., Keegstra, W., Boekema, E.J., and Braun, H.-P. (2005). Structure of dimeric ATP synthase from mitochondria: An angular association of monomers induces the strong curvature of the inner membrane. Febs Lett 579, 5769-5772.

Duvezin-Caubet, S., Jagasia, R., Wagener, J., Hofmann, S., Trifunovic, A., Hansson, A., Chomyn, A., Bauer, M.F., Attardi, G., Larsson, N.-G., et al. (2006). Proteolytic Processing of OPA1 Links Mitochondrial Dysfunction to Alterations in Mitochondrial Morphology. J Biol Chem 281, 37972-37979. 
Eaton, S., Bartlett, K.B., and Pourfazam, M. (1996). Mammalian mitochondrial $\beta$-oxidation. Biochem J 320, 345-357.

Elachouri, G., Vidoni, S., Zanna, C., Pattyn, A., Boukhaddaoui, H., Gaget, K., Yu-Wai-Man, P., Gasparre, G., Sarzi, E., Delettre, C., et al. (2010). OPA1 links human mitochondrial genome maintenance to mtDNA replication and distribution. Genome Res 21, 12-20.

Esser, K., Tursun, B., Ingenhoven, M., Michaelis, G., and Pratje, E. (2002). A Novel Two-step Mechanism for Removal of a Mitochondrial Signal Sequence Involves the mAAA Complex and the Putative Rhomboid Protease Pcp1. J Mol Biol 323, 835-843.

Eura, Y., Ishihara, N., Yokota, S., and Mihara, K. (2003). Two Mitofusin Proteins, Mammalian Homologues of FZO, with Distinct Functions Are Both Required for Mitochondrial Fusion. J Biochem 134, 333-344.

Eydt, K., Davies, K.M., Behrendt, C., Wittig, I., and Reichert, A.S. (2017). Cristae architecture is determined by an interplay of the MICOS complex and the F1FO ATP synthase via Mic27 and Mic10. Microb Cell 4, 259-272.

Faelber, K., Dietrich, L., Noel, J.K., Wollweber, F., Pfitzner, A.-K., Mühleip, A., Sánchez, R., Kudryashev, M., Chiaruttini, N., Lilie, H., et al. (2019). Structure and assembly of the mitochondrial membrane remodelling GTPase Mgm1. Nature 571, 429-433.

Fehrenbacher, K.L., Yang, H.-C., Gay, A.C., Huckaba, T.M., and Pon, L.A. (2004). Live Cell Imaging of Mitochondrial Movement along Actin Cables in Budding Yeast. Curr Biol 14, 1996-2004.

Filadi, R., Greotti, E., Turacchio, G., Luini, A., Pozzan, T., and Pizzo, P. (2015). Mitofusin 2 ablation increases endoplasmic reticulum-mitochondria coupling. P Natl Acad Sci Usa 112, E2174-81.

Fox, T.D. (2012). Mitochondrial protein synthesis, import, and assembly. Genetics 192, 1203-1234.

Frey, T.G., Renken, C.W., and Perkins, G.A. (2002). Insight into mitochondrial structure and function from electron tomography. Biochimica Et Biophysica Acta Bba - Bioenergetics 1555, 196-203.

Frezza, C., Cipolat, S., Brito, O.M. de, Micaroni, M., Beznoussenko, G.V., Rudka, T., Bartoli, D., Polishuck, R.S., Danial, N.N., Strooper, B.D., et al. (2006). OPA1 Controls Apoptotic Cristae Remodeling Independently from Mitochondrial Fusion. Cell 126, 177-189.

Friedman, J.R., and Nunnari, J. (2014). Mitochondrial form and function. Nature 505, 335-343.

Friedman, J.R., Lackner, L.L., West, M., DiBenedetto, J.R., Nunnari, J., and Voeltz, G.K. (2011). ER tubules mark sites of mitochondrial division. Sci New York N Y 334, 358-362.

Friedman, J.R., Mourier, A., Yamada, J., McCaffery, J.M., and Nunnari, J. (2015). MICOS coordinates with respiratory complexes and lipids to establish mitochondrial inner membrane architecture. Elife 4 , e07739.

Friedman, J.R., Kannan, M., Toulmay, A., Jan, C.H., Weissman, J.S., Prinz, W.A., and Nunnari, J. (2018). Lipid Homeostasis Is Maintained by Dual Targeting of the Mitochondrial PE Biosynthesis Enzyme to the ER. Dev Cell 44, 261-270.e6.

Fry, M., and Green, D.E. (1981). Cardiolipin requirement for electron transfer in complex I and III of the mitochondrial respiratory chain. J Biological Chem 256, 1874-1880.

Ge, Y., Shi, X., Boopathy, S., McDonald, J., Smith, A.W., and Chao, L.H. (2020). Two forms of Opa1 cooperate to complete fusion of the mitochondrial inner-membrane. Elife 9, e50973.

Ghochani, M., Nulton, J.D., Salamon, P., Frey, T.G., Rabinovitch, A., and Baljon, A.R.C. (2010). Tensile forces and shape entropy explain observed crista structure in mitochondria. Biophys J 99, 3244-3254. 
Giacomello, M., Pyakurel, A., Glytsou, C., and Scorrano, L. (2020). The cell biology of mitochondrial membrane dynamics. Nat Rev Mol Cell Biology 1-21.

Glater, E.E., Megeath, L.J., Stowers, R.S., and Schwarz, T.L. (2006). Axonal transport of mitochondria requires milton to recruit kinesin heavy chain and is light chain independent. J Cell Biology 173, 545557.

Glytsou, C., Calvo, E., Cogliati, S., Mehrotra, A., Anastasia, I., Rigoni, G., Raimondi, A., Shintani, N., Loureiro, M., Vazquez, J., et al. (2016). Optic Atrophy 1 Is Epistatic to the Core MICOS Component MIC60 in Mitochondrial Cristae Shape Control. Cell Reports 17, 3024-3034.

Gray, M.W., Burger, G., and Lang, B.F. (1999). Mitochondrial Evolution. Science 283, 1476-1481.

Griparic, L., Wel, N.N. van der, Orozco, I.J., Peters, P.J., and Bliek, A.M. van der (2004). Loss of the Intermembrane Space Protein Mgm1/OPA1 Induces Swelling and Localized Constrictions along the Lengths of Mitochondria. J Biol Chem 279, 18792-18798.

Griparic, L., Kanazawa, T., and Bliek, A.M. van der (2007). Regulation of the mitochondrial dynamin-like protein Opa1 by proteolytic cleavage. J Cell Biology 178, 757-764.

Große, L., Wurm, C.A., Brüser, C., Neumann, D., Jans, D.C., and Jakobs, S. (2016). Bax assembles into large ring-like structures remodeling the mitochondrial outer membrane in apoptosis. Embo $\mathrm{J} 35$, 402-413.

Grotjohann, T., Testa, I., Leutenegger, M., Bock, H., Urban, N.T., Lavoie-Cardinal, F., Willig, K.I., Eggeling, C., Jakobs, S., and Hell, S.W. (2011). Diffraction-unlimited all-optical imaging and writing with a photochromic GFP. Nature 478, 204-208.

Gu, J., Zhang, L., Zong, S., Guo, R., Liu, T., Yi, J., Wang, P., Zhuo, W., and Yang, M. (2019). Cryo-EM structure of the mammalian ATP synthase tetramer bound with inhibitory protein IF1. Sci New York N Y $364,1068-1075$.

Guan, K., Farh, L., Marshall, T.K., and Deschenes, R.J. (1993). Normal mitochondrial structure and genome maintenance in yeast requires the dynamin-like product of the MGM1 gene. Curr Genet 24, $141-148$.

Guarani, V., McNeill, E.M., Paulo, J.A., Huttlin, E.L., Fröhlich, F., Gygi, S.P., Vactor, D.V., and Harper, J.W. (2015). QIL1 is a novel mitochondrial protein required for MICOS complex stability and cristae morphology. Elife 4, e06265.

Guo, Y., Li, D., Zhang, S., Yang, Y., Liu, J.-J., Wang, X., Liu, C., Milkie, D.E., Moore, R.P., Tulu, U.S., et al. (2018). Visualizing Intracellular Organelle and Cytoskeletal Interactions at Nanoscale Resolution on Millisecond Timescales. Cell 175, 1430-1442.e17.

Gustafsson, M.G.L. (2000). Surpassing the lateral resolution limit by a factor of two using structured illumination microscopy. SHORT COMMUNICATION. J Microsc-Oxford 198, 82-87.

Gustafsson, M.G.L. (2005). Nonlinear structured-illumination microscopy: Wide-field fluorescence imaging with theoretically unlimited resolution. Proc National Acad Sci 102, 13081-13086.

Hackenbrock, C.R. (1966). Ultrastructural bases for metabolically linked mechanical activity in mitochondria. J Cell Biology 30, 269-297.

Hahn, A., Parey, K., Bublitz, M., Mills, D.J., Zickermann, V., Vonck, J., Kühlbrandt, W., and Meier, T. (2016). Structure of a Complete ATP Synthase Dimer Reveals the Molecular Basis of Inner Mitochondrial Membrane Morphology. Mol Cell 63, 445-456.

Hales, K.G., and Fuller, M.T. (1997). Developmentally Regulated Mitochondrial Fusion Mediated by a Conserved, Novel, Predicted GTPase. Cell 90, 121-129. 
Harner, M., Körner, C., Walther, D., Mokranjac, D., Kaesmacher, J., Welsch, U., Griffith, J., Mann, M., Reggiori, F., and Neupert, W. (2011). The mitochondrial contact site complex, a determinant of mitochondrial architecture. Embo J 30, 4356-4370.

Harner, M.E., Unger, A.-K., Izawa, T., Walther, D.M., Ozbalci, C., Geimer, S., Reggiori, F., Brügger, B., Mann, M., Westermann, B., et al. (2014). Aim24 and MICOS modulate respiratory function, tafazzinrelated cardiolipin modification and mitochondrial architecture. Elife 3, e01684.

Harner, M.E., Unger, A.-K., Geerts, W.J., Mari, M., Izawa, T., Stenger, M., Geimer, S., Reggiori, F., Westermann, B., and Neupert, W. (2016). An evidence based hypothesis on the existence of two pathways of mitochondrial crista formation. Elife 5, e18853.

Hell, S.W. (2005). Fluorescence nanoscopy: Breaking the diffraction barrier by the RESOLFT concept. Nanobiotechnology 1, 296-297.

Hell, S.W., and Wichmann, J. (1994). Breaking the diffraction resolution limit by stimulated emission: stimulated-emission-depletion fluorescence microscopy. Opt Lett 19, 780.

Herlan, M., Vogel, F., Bornhövd, C., Neupert, W., and Reichert, A.S. (2003). Processing of Mgm1 by the Rhomboid-type Protease Pcp1 is Required for Maintenance of Mitochondrial Morphology and of Mitochondrial DNA. J Biol Chem 278, 27781-27788.

Herlan, M., Bornhövd, C., Hell, K., Neupert, W., and Reichert, A.S. (2004). Alternative topogenesis of Mgm1 and mitochondrial morphology depend on ATP and a functional import motor. J Cell Biology 165, 167-173.

Hermann, G.J., Thatcher, J.W., Mills, J.P., Hales, K.G., Fuller, M.T., Nunnari, J., and Shaw, J.M. (1998). Mitochondrial Fusion in Yeast Requires the Transmembrane GTPase Fzo1p. J Cell Biology 143, 359373.

Hessenberger, M., Zerbes, R.M., Rampelt, H., Kunz, S., Xavier, A.H., Purfürst, B., Lilie, H., Pfanner, N., Laan, M. van der, and Daumke, O. (2017). Regulated membrane remodeling by Mic60 controls formation of mitochondrial crista junctions. Nat Commun 8, 15258.

Hill, K., Model, K., Ryan, M.T., Dietmeier, K., Martin, F., Wagner, R., and Pfanner, N. (1998). Tom40 forms the hydrophilic channel of the mitochondrial import pore for preproteins. Nature 395, 516-521.

Höhr, A.I.C., Straub, S.P., Warscheid, B., Becker, T., and Wiedemann, N. (2014). Assembly of $\beta$-barrel proteins in the mitochondrial outer membrane. Biochim Biophys Acta 1853, 74-88.

Holt, I.J., and Reyes, A. (2012). Human mitochondrial DNA replication. Csh Perspect Biol 4, a012971a012971.

Hoppins, S., Collins, S.R., Cassidy-Stone, A., Hummel, E., Devay, R.M., Lackner, L.L., Westermann, B., Schuldiner, M., Weissman, J.S., and Nunnari, J. (2011). A mitochondrial-focused genetic interaction map reveals a scaffold-like complex required for inner membrane organization in mitochondria. J Cell Biology 195, 323-340.

Horvath, S.E., Rampelt, H., Oeljeklaus, S., Warscheid, B., Laan, M. van der, and Pfanner, N. (2015). Role of membrane contact sites in protein import into mitochondria. Protein Sci Publ Protein Soc 24, 277-297.

Huang, X., Fan, J., Li, L., Liu, H., Wu, R., Wu, Y., Wei, L., Mao, H., Lal, A., Xi, P., et al. (2018). Fast, long-term, super-resolution imaging with Hessian structured illumination microscopy. Nat Biotechnol 36, $451-459$.

Huynen, M.A., Mühlmeister, M., Gotthardt, K., Guerrero-Castillo, S., and Brandt, U. (2015). Evolution and structural organization of the mitochondrial contact site (MICOS) complex and the mitochondrial intermembrane space bridging (MIB) complex. Biochim Biophys Acta 1863, 91-101. 
Ishihara, N., Eura, Y., and Mihara, K. (2004). Mitofusin 1 and 2 play distinct roles in mitochondrial fusion reactions via GTPase activity. J Cell Sci 117, 6535-6546.

Ishihara, N., Fujita, Y., Oka, T., and Mihara, K. (2006). Regulation of mitochondrial morphology through proteolytic cleavage of OPA1. Embo J 25, 2966-2977.

Jakobs, S., Stephan, T., Ilgen, P., and Brüser, C. (2020). Light Microscopy of Mitochondria at the Nanoscale. Annu. Rev. Biophys.

Jans, D.C., Wurm, C.A., Riedel, D., Wenzel, D., Stagge, F., Deckers, M., Rehling, P., and Jakobs, S. (2013). STED super-resolution microscopy reveals an array of MINOS clusters along human mitochondria. P Natl Acad Sci Usa 110, 8936-8941.

Ji, W., Hatch, A.L., Merrill, R.A., Strack, S., and Higgs, H.N. (2015). Actin filaments target the oligomeric maturation of the dynamin GTPase Drp1 to mitochondrial fission sites. Elife 4, e11553.

Jiang, Y.-F., Lin, H.-L., Wang, L.-J., Hsu, T., and Fu, C.-Y. (2019). Coordinated organization of mitochondrial lamellar cristae and gain of COX function during mitochondrial maturation in Drosophila. Mol Biol Cell 31, 18-26.

John, G.B., Shang, Y., Li, L., Renken, C., Mannella, C.A., Selker, J.M.L., Rangell, L., Bennett, M.J., and Zha, J. (2005). The Mitochondrial Inner Membrane Protein Mitofilin Controls Cristae Morphology. Mol Biol Cell 16, 1543-1554.

Jones, B.A., and Fangman, W.L. (1992). Mitochondrial DNA maintenance in yeast requires a protein containing a region related to the GTP-binding domain of dynamin. Gene Dev 6, 380-389.

Juillerat, A., Gronemeyer, T., Keppler, A., Gendreizig, S., Pick, H., Vogel, H., and Johnsson, K. (2003). Directed Evolution of O6-Alkylguanine-DNA Alkyltransferase for Efficient Labeling of Fusion Proteins with Small Molecules In Vivo. Chem Biol 10, 313-317.

Kadenbach, B. (2012). Advances in Experimental Medicine and Biology. Adv Exp Med Biol 748, 1-11.

Kalkavan, H., and Green, D.R. (2017). MOMP, cell suicide as a BCL-2 family business. Cell Death Differ $25,46-55$.

Khalifat, N., Puff, N., Bonneau, S., Fournier, J.-B., and Angelova, M.I. (2008). Membrane deformation under local pH gradient: mimicking mitochondrial cristae dynamics. Biophys J 95, 4924-4933.

Khalifat, N., Fournier, J.-B., Angelova, M.I., and Puff, N. (2011). Lipid packing variations induced by pH in cardiolipin-containing bilayers: The driving force for the cristae-like shape instability. Biochimica Et Biophysica Acta Bba - Biomembr 1808, 2724-2733.

Kiebler, M., Pfaller, R., Söllner, T., Griffiths, G., Horstmann, H., Pfanner, N., and Neupert, W. (1990). Identification of a mitochondrial receptor complex required for recognition and membrane insertion of precursor proteins. Nature 348, 610-616.

Kilian, N., Goryaynov, A., Lessard, M.D., Hooker, G., Toomre, D., Rothman, J.E., and Bewersdorf, J. (2018). Assessing photodamage in live-cell STED microscopy. Nat Methods 15, 755-756.

Klar, T.A., Jakobs, S., Dyba, M., Egner, A., and Hell, S.W. (2000). Fluorescence microscopy with diffraction resolution barrier broken by stimulated emission. Proc National Acad Sci 97, 8206-8210.

Klein, A., Israel, L., Lackey, S.W.K., Nargang, F.E., Imhof, A., Baumeister, W., Neupert, W., and Thomas, D.R. (2012). Characterization of the insertase for $\beta$-barrel proteins of the outer mitochondrial membrane. J Cell Biology 199, 599-611.

Kojima, R., Kakimoto, Y., Furuta, S., Itoh, K., Sesaki, H., Endo, T., and Tamura, Y. (2019). Maintenance of Cardiolipin and Crista Structure Requires Cooperative Functions of Mitochondrial Dynamics and Phospholipid Transport. Cell Reports 26, 518-528.e6. 
Kölliker, A. von (1856). Zeitschrift für wissenschaftliche Zoologie 8, 311-318.

Kondadi, A.K., Anand, R., and Reichert, A.S. (2019). Functional Interplay between Cristae Biogenesis, Mitochondrial Dynamics and Mitochondrial DNA Integrity. Int J Mol Sci 20, 4311.

Kondadi, A.K., Anand, R., Hänsch, S., Urbach, J., Zobel, T., Wolf, D.M., Segawa, M., Liesa, M., Shirihai, O.S., Weidtkamp-Peters, S., et al. (2020). Cristae undergo continuous cycles of membrane remodelling in a MICOS-dependent manner. Embo Rep e49776.

Koob, S., Barrera, M., Anand, R., and Reichert, A.S. (2015). The non-glycosylated isoform of MIC26 is a constituent of the mammalian MICOS complex and promotes formation of crista junctions. Biochimica Et Biophysica Acta Bba - Mol Cell Res 1853, 1551-1563.

Körner, C., Barrera, M., Dukanovic, J., Eydt, K., Harner, M., Rabl, R., Vogel, F., Rapaport, D., Neupert, W., and Reichert, A.S. (2012). The C-terminal domain of Fcj1 is required for formation of crista junctions and interacts with the TOB/SAM complex in mitochondria. Mol Biol Cell 23, 2143-2155.

Kornmann, B., Currie, E., Collins, S.R., Schuldiner, M., Nunnari, J., Weissman, J.S., and Walter, P. (2009). An ER-mitochondria tethering complex revealed by a synthetic biology screen. Sci New York N Y 325, 477-481.

Korobova, F., Ramabhadran, V., and Higgs, H.N. (2013). An actin-dependent step in mitochondrial fission mediated by the ER-associated formin INF2. Sci New York N Y 339, 464-467.

Kühlbrandt, W. (2019). Structure and Mechanisms of F-Type ATP Synthases. Annu Rev Biochem 88, $515-549$.

Laan, M. van der, Horvath, S.E., and Pfanner, N. (2016). Mitochondrial contact site and cristae organizing system. Curr Opin Cell Biol 41, 33-42.

Lea, P.J., and Hollenberg, M.J. (1989). Mitochondrial structure revealed by high-resolution scanning electron microscopy. Am J Anat 184, 245-257.

Lee, H., Smith, S.B., and Yoon, Y. (2017). The short variant of the mitochondrial dynamin OPA1 maintains mitochondrial energetics and cristae structure. J Biological Chem 292, 7115-7130.

Lee, Y., Jeong, S.-Y., Karbowski, M., Smith, C.L., and Youle, R.J. (2004). Roles of the Mammalian Mitochondrial Fission and Fusion Mediators Fis1, Drp1, and Opa1 in Apoptosis. Mol Biol Cell 15, 50015011.

Legros, F., Lombès, A., Frachon, P., and Rojo, M. (2002). Mitochondrial Fusion in Human Cells Is Efficient, Requires the Inner Membrane Potential, and Is Mediated by Mitofusins. Mol Biol Cell 13, 43434354.

Li, H., Ruan, Y., Zhang, K., Jian, F., Hu, C., Miao, L., Gong, L., Sun, L., Zhang, X., Chen, S., et al. (2015). Mic60/Mitofilin determines MICOS assembly essential for mitochondrial dynamics and mtDNA nucleoid organization. Cell Death Differ 23, 380-392.

Lukinavičius, G., Umezawa, K., Olivier, N., Honigmann, A., Yang, G., Plass, T., Mueller, V., Reymond, L., Corrêa, I.R., Luo, Z.-G., et al. (2013). A near-infrared fluorophore for live-cell super-resolution microscopy of cellular proteins. Nat Chem 5, 132-139.

MacVicar, T., and Langer, T. (2016). OPA1 processing in cell death and disease - the long and short of it. J Cell Sci 129, 2297-2306.

Mai, N., Chrzanowska-Lightowlers, Z.M.A., and Lightowlers, R.N. (2016). The process of mammalian mitochondrial protein synthesis. Cell Tissue Res 367, 5-20. 
Malsburg, K. von der, Müller, J.M., Bohnert, M., Oeljeklaus, S., Kwiatkowska, P., Becker, T., LoniewskaLwowska, A., Wiese, S., Rao, S., Milenkovic, D., et al. (2011). Dual role of mitofilin in mitochondrial membrane organization and protein biogenesis. Dev Cell 21, 694-707.

Mannella, C.A. (1992). The 'ins' and 'outs' of mitochondrial membrane channels. Trends Biochem Sci 17, 315-320.

Mannella, C.A. (2006). Structure and dynamics of the mitochondrial inner membrane cristae. Biochimica Et Biophysica Acta Bba - Mol Cell Res 1763, 542-548.

Mannella, C.A., Marko, M., Penczek, P., Barnard, D., and Frank, J. (1994). The internal compartmentation of rat-liver mitochondria: Tomographic study using the high-voltage transmission electron microscope. Microsc Res Techniq 27, 278-283.

Mannella, C.A., Pfeiffer, D.R., Bradshaw, P.C., Moraru, I.I., Slepchenko, B., Loew, L.M., Hsieh, C., Buttle, K., and Marko, M. (2001). Topology of the Mitochondrial Inner Membrane: Dynamics and Bioenergetic Implications. Iubmb Life Int Union Biochem Mol Biology Life 52, 93-100.

Manor, U., Bartholomew, S., Golani, G., Christenson, E., Kozlov, M., Higgs, H., Spudich, J., and Lippincott-Schwartz, J. (2015). A mitochondria-anchored isoform of the actin-nucleating spire protein regulates mitochondrial division. Elife 4 , e08828.

McArthur, K., Whitehead, L.W., Heddleston, J.M., Li, L., Padman, B.S., Oorschot, V., Geoghegan, N.D., Chappaz, S., Davidson, S., Chin, H.S., et al. (2018). BAK/BAX macropores facilitate mitochondrial herniation and mtDNA efflux during apoptosis. Sci New York N Y 359, eaao6047.

McQuibban, G.A., Saurya, S., and Freeman, M. (2003). Mitochondrial membrane remodelling regulated by a conserved rhomboid protease. Nature $423,537-541$.

Meeusen, S., McCaffery, J.M., and Nunnari, J. (2004). Mitochondrial Fusion Intermediates Revealed in Vitro. Science 305, 1747-1752.

Meeusen, S., DeVay, R., Block, J., Cassidy-Stone, A., Wayson, S., McCaffery, J.M., and Nunnari, J. (2006). Mitochondrial Inner-Membrane Fusion and Crista Maintenance Requires the Dynamin-Related GTPase Mgm1. Cell 127, 383-395.

Meglei, G., and McQuibban, G.A. (2009). The Dynamin-Related Protein Mgm1p Assembles into Oligomers and Hydrolyzes GTP To Function in Mitochondrial Membrane Fusion †. Biochemistry-Us 48, 1774-1784.

Mereschkowsky, C. (1910). Theorie der zwei Plasmaarten als Grundlage der Symbiogenesis, einer neuen Lehre von der Entstehung der Organismen. Biologisches Centralblatt 30, 278-288;289-303; 322374; 353-367.

Mitchell, P. (1961). Coupling of Phosphorylation to Electron and Hydrogen Transfer by a Chemi-Osmotic type of Mechanism. Nature 191, 144-148.

Moczko, M., Bömer, U., Kübrich, M., Zufall, N., Hönlinger, A., and Pfanner, N. (1997). The intermembrane space domain of mitochondrial Tom22 functions as a trans binding site for preproteins with N-terminal targeting sequences. Mol Cell Biol 17, 6574-6584.

Modi, S., López-Doménech, G., Halff, E.F., Covill-Cooke, C., Ivankovic, D., Melandri, D., ArancibiaCárcamo, I.L., Burden, J.J., Lowe, A.R., and Kittler, J.T. (2019). Miro clusters regulate ER-mitochondria contact sites and link cristae organization to the mitochondrial transport machinery. Nat Commun 10, 4399 .

Mokranjac, D., and Neupert, W. (2015). Cell biology: Architecture of a protein entry gate. Nature 528, 201-202. 
Morris, R.L., and Hollenbeck, P.J. (1995). Axonal transport of mitochondria along microtubules and Factin in living vertebrate neurons. J Cell Biology 131, 1315-1326.

Moualij, B.E., Duyckaerts, C., Lamotte-Brasseur, J., and Sluse, F.E. (1997). Phylogenetic Classification of the Mitochondrial Carrier Family ofSaccharomyces cerevisiae. Yeast 13, 573-581.

Mühleip, A.W., Joos, F., Wigge, C., Frangakis, A.S., Kühlbrandt, W., and Davies, K.M. (2016). Helical arrays of U-shaped ATP synthase dimers form tubular cristae in ciliate mitochondria. P Natl Acad Sci Usa 113, 8442-8447.

Müller, C.B., and Enderlein, J. (2010). Image Scanning Microscopy. Phys Rev Lett 104, 198101.

Mun, J.Y., Lee, T.H., Kim, J.H., Yoo, B.H., Bahk, Y.Y., Koo, H.-S., and Han, S.S. (2010). Caenorhabditis elegans mitofilin homologs control the morphology of mitochondrial cristae and influence reproduction and physiology. J Cell Physiol 224, 748-756.

Munn, E.A. (1974). The Structure of Mitochondria. 107-138.

Muñoz-Gómez, S.A., Slamovits, C.H., Dacks, J.B., Baier, K.A., Spencer, K.D., and Wideman, J.G. (2015). Ancient homology of the mitochondrial contact site and cristae organizing system points to an endosymbiotic origin of mitochondrial cristae. Curr Biology $\mathrm{Cb} 25,1489-1495$.

Muñoz-Gómez, S.A., Wideman, J.G., Roger, A.J., and Slamovits, C.H. (2017). The origin of mitochondrial cristae from alphaproteobacteria. Mol Biol Evol msw298.

Murphy, B.J., Klusch, N., Langer, J., Mills, D.J., Yildiz, Ö., and Kühlbrandt, W. (2019). Rotary substates of mitochondrial ATP synthase reveal the basis of flexible F 1 -F o coupling. Science 364, eaaw9128.

Naon, D., Zaninello, M., Giacomello, M., Varanita, T., Grespi, F., Lakshminaranayan, S., Serafini, A., Semenzato, M., Herkenne, S., Hernández-Alvarez, M.I., et al. (2017). Reply to Filadi et al.: Does Mitofusin 2 tether or separate endoplasmic reticulum and mitochondria? P Natl Acad Sci Usa 114, E2268-E2269.

Nass, M.M.K., and Nass, S. (1963). Intramitochondrial fibers with DNA characteristics. J Cell Biology $19,593-611$.

Oeding, S.J., Majstrowicz, K., Hu, X.-P., Schwarz, V., Freitag, A., Honnert, U., Nikolaus, P., and Bähler, M. (2018). Identification of Miro1 and Miro2 as mitochondrial receptors for myosin XIX. J Cell Sci 131, jcs219469.

Olichon, A., Baricault, L., Gas, N., Guillou, E., Valette, A., Belenguer, P., and Lenaers, G. (2002). Loss of OPA1 Perturbates the Mitochondrial Inner Membrane Structure and Integrity, Leading to Cytochrome c Release and Apoptosis. J Biol Chem 278, 7743-7746.

Ott, C., Ross, K., Straub, S., Thiede, B., Götz, M., Goosmann, C., Krischke, M., Mueller, M.J., Krohne, G., Rudel, T., et al. (2012). Sam50 functions in mitochondrial intermembrane space bridging and biogenesis of respiratory complexes. Mol Cell Biol 32, 1173-1188.

Ott, C., Dorsch, E., Fraunholz, M., Straub, S., and Kozjak-Pavlovic, V. (2015). Detailed analysis of the human mitochondrial contact site complex indicate a hierarchy of subunits. Plos One 10, e0120213.

Palade, G.E. (1952). The fine structure of mitochondria. Anatomical Rec 114, 427-451.

Paschen, S.A., Waizenegger, T., Stan, T., Preuss, M., Cyrklaff, M., Hell, K., Rapaport, D., and Neupert, W. (2003). Evolutionary conservation of biogenesis of $\beta$-barrel membrane proteins. Nature 426, $862-$ 866.

Patten, D.A., Wong, J., Khacho, M., Soubannier, V., Mailloux, R.J., Pilon-Larose, K., MacLaurin, J.G., Park, D.S., McBride, H.M., Trinkle-Mulcahy, L., et al. (2014). OPA1-dependent cristae modulation is essential for cellular adaptation to metabolic demand. Embo J 33, 2676-2691. 
Paumard, P., Vaillier, J., Coulary, B., Schaeffer, J., Soubannier, V., Mueller, D.M., Brèthes, D., Rago, J.-P. di, and Velours, J. (2002). The ATP synthase is involved in generating mitochondrial cristae morphology. Embo J 21, 221-230.

Perkins, G., Renken, C., Martone, M.E., Young, S.J., Ellisman, M., and Frey, T. (1997). Electron Tomography of Neuronal Mitochondria: Three-Dimensional Structure and Organization of Cristae and Membrane Contacts. J Struct Biol 119, 260-272.

Pernas, L., and Scorrano, L. (2016). Mito-Morphosis: Mitochondrial Fusion, Fission, and Cristae Remodeling as Key Mediators of Cellular Function. Annu Rev Physiol 78, 505-531.

Pfanner, N., Laan, M. van der, Amati, P., Capaldi, R.A., Caudy, A.A., Chacinska, A., Darshi, M., Deckers, M., Hoppins, S., Icho, T., et al. (2014). Uniform nomenclature for the mitochondrial contact site and cristae organizing system. J Cell Biology 204, 1083-1086.

Qi, Y., Yan, L., Yu, C., Guo, X., Zhou, X., Hu, X., Huang, X., Rao, Z., Lou, Z., and Hu, J. (2016). Structures of human mitofusin 1 provide insight into mitochondrial tethering. J Cell Biology 215, 621629.

Quintana-Cabrera, R., Quirin, C., Glytsou, C., Corrado, M., Urbani, A., Pellattiero, A., Calvo, E., Vázquez, J., Enríquez, J.A., Gerle, C., et al. (2018). The cristae modulator Optic atrophy 1 requires mitochondrial ATP synthase oligomers to safeguard mitochondrial function. Nat Commun 9, 3399.

Quintero, O.A., DiVito, M.M., Adikes, R.C., Kortan, M.B., Case, L.B., Lier, A.J., Panaretos, N.S., Slater, S.Q., Rengarajan, M., Feliu, M., et al. (2009). Human Myo19 is a novel myosin that associates with mitochondria. Curr Biology Cb 19, 2008-2013.

Rabl, R., Soubannier, V., Scholz, R., Vogel, F., Mendl, N., Vasiljev-Neumeyer, A., Körner, C., Jagasia, R., Keil, T., Baumeister, W., et al. (2009). Formation of cristae and crista junctions in mitochondria depends on antagonism between Fcj1 and Su e/g. J Cell Biology 185, 1047-1063.

Rampelt, H., Zerbes, R.M., Laan, M. van der, and Pfanner, N. (2017a). Role of the mitochondrial contact site and cristae organizing system in membrane architecture and dynamics. Biochimica Et Biophysica Acta Bba - Mol Cell Res 1864, 737-746.

Rampelt, H., Bohnert, M., Zerbes, R.M., Horvath, S.E., Warscheid, B., Pfanner, N., and Laan, M. van $\operatorname{der}$ (2017b). Mic10, a Core Subunit of the Mitochondrial Contact Site and Cristae Organizing System, Interacts with the Dimeric F1Fo-ATP Synthase. J Mol Biol 429, 1162-1170.

Rapaport, D., Brunner, M., Neupert, W., and Westermann, B. (1998). Fzo1p Is a Mitochondrial Outer Membrane Protein Essential for the Biogenesis of Functional Mitochondria in Saccharomyces cerevisiae. J Biol Chem 273, 20150-20155.

Renken, C., Siragusa, G., Perkins, G., Washington, L., Nulton, J., Salamon, P., and Frey, T.G. (2002). A thermodynamic model describing the nature of the crista junction: a structural motif in the mitochondrion. J Struct Biol 138, 137-144.

Riley, J.S., Quarato, G., Cloix, C., Lopez, J., O’Prey, J., Pearson, M., Chapman, J., Sesaki, H., Carlin, L.M., Passos, J.F., et al. (2018). Mitochondrial inner membrane permeabilisation enables mt DNA release during apoptosis. Embo $\mathrm{J} 37$.

Rojo, M., Legros, F., Chateau, D., and Lombès, A. (2002). Membrane topology and mitochondrial targeting of mitofusins, ubiquitous mammalian homologs of the transmembrane GTPase Fzo. J Cell Sci $115,1663-1674$.

Rusiñol, A.E., Cui, Z., Chen, M.H., and Vance, J.E. (1994). A unique mitochondria-associated membrane fraction from rat liver has a high capacity for lipid synthesis and contains pre-Golgi secretory proteins including nascent lipoproteins. J Biological Chem 269, 27494-27502. 
Rust, M.J., Bates, M., and Zhuang, X. (2006). Sub-diffraction-limit imaging by stochastic optical reconstruction microscopy (STORM). Nat Methods 3, 793-796.

Sagan, L. (1967). On the origin of mitosing cells. J Theor Biol 14, 225-IN6.

Sahl, S.J., Hell, S.W., and Jakobs, S. (2017). Fluorescence nanoscopy in cell biology. Nat Rev Mol Cell Biology 18, 685-701.

Sakowska, P., Jans, D.C., Mohanraj, K., Riedel, D., Jakobs, S., and Chacinska, A. (2015). The Oxidation Status of Mic19 Regulates MICOS Assembly. Mol Cell Biol 35, 4222-4237.

Salvador-Gallego, R., Mund, M., Cosentino, K., Schneider, J., Unsay, J., Schraermeyer, U., Engelhardt, J., Ries, J., and García-Sáez, A.J. (2016). Bax assembly into rings and arcs in apoptotic mitochondria is linked to membrane pores. Embo J 35, 389-401.

Santel, A., and Fuller, M.T. (2001). Control of mitochondrial morphology by a human mitofusin. J Cell Sci 114, 867-874.

Scheffler, I.E. (2007). Mitochondria. i-xviii.

Senior, A.E., Nadanaciva, S., and Weber, J. (2002). The molecular mechanism of ATP synthesis by F1F0-ATP synthase. Biochimica Et Biophysica Acta Bba - Bioenergetics 1553, 188-211.

Sesaki, H., Southard, S.M., Yaffe, M.P., and Jensen, R.E. (2003). Mgm1p, a Dynamin-related GTPase, Is Essential for Fusion of the Mitochondrial Outer Membrane. Mol Biol Cell 14, 2342-2356.

Shai, N., Yifrach, E., Roermund, C.W.T. van, Cohen, N., Bibi, C., IJlst, L., Cavellini, L., Meurisse, J., Schuster, R., Zada, L., et al. (2018). Systematic mapping of contact sites reveals tethers and a function for the peroxisome-mitochondria contact. Nat Commun 9, 1761.

Shepard, K.A., and Yaffe, M.P. (1999). The Yeast Dynamin-like Protein, Mgm1p, Functions on the Mitochondrial Outer Membrane to Mediate Mitochondrial Inheritance. J Cell Biology 144, 711-720.

Shiota, T., Imai, K., Qiu, J., Hewitt, V.L., Tan, K., Shen, H.-H., Sakiyama, N., Fukasawa, Y., Hayat, S., Kamiya, M., et al. (2015). Molecular architecture of the active mitochondrial protein gate. Science 349, 1544-1548.

Signes, A., and Fernandez-Vizarra, E. (2018). Assembly of mammalian oxidative phosphorylation complexes I-V and supercomplexes. Essays Biochem 62, 255-270.

Simbeni, R., Pon, L., Zinser, E., Paltauf, F., and Daum, G. (1991). Mitochondrial membrane contact sites of yeast. Characterization of lipid components and possible involvement in intramitochondrial translocation of phospholipids. J Biological Chem 266, 10047-10049.

Sirrenberg, C., Bauer, M.F., Guiard, B., Neupert, W., and Brunner, M. (1996). Import of carrier proteins into the mitochondrial inner membrane mediated by Tim22. Nature $384,582-585$.

Sjöstrand, F.S. (1953). Electron Microscopy of Mitochondria and Cytoplasmic Double Membranes: UltraStructure of Rod-shaped Mitochondria. Nature 171, 30-31.

Song, Z., Chen, H., Fiket, M., Alexander, C., and Chan, D.C. (2007). OPA1 processing controls mitochondrial fusion and is regulated by mRNA splicing, membrane potential, and Yme1L. J Cell Biology 178, 749-755.

Strauss, M., Hofhaus, G., Schröder, R.R., and Kühlbrandt, W. (2008). Dimer ribbons of ATP synthase shape the inner mitochondrial membrane. Embo J 27, 1154-1160.

Sugiura, A., Nagashima, S., Tokuyama, T., Amo, T., Matsuki, Y., Ishido, S., Kudo, Y., McBride, H.M., Fukuda, T., Matsushita, N., et al. (2013). MITOL regulates endoplasmic reticulum-mitochondria contacts via Mitofusin2. Mol Cell 51, 20-34. 
Tang, J., Zhang, K., Dong, J., Yan, C., Chen, S., and Song, Z. (2018). Sam50-Mic19-Mic60 axis Determines Mitochondrial Cristae Architecture by Mediating Mitochondrial Outer and Inner Membrane Contact. Biorxiv 345959.

Tang, J., Zhang, K., Dong, J., Yan, C., Hu, C., Ji, H., Chen, L., Chen, S., Zhao, H., and Song, Z. (2019). Sam50-Mic19-Mic60 axis determines mitochondrial cristae architecture by mediating mitochondrial outer and inner membrane contact. Cell Death Differ 27, 146-160.

Tarasenko, D., Barbot, M., Jans, D.C., Kroppen, B., Sadowski, B., Heim, G., Möbius, W., Jakobs, S., and Meinecke, M. (2017). The MICOS component Mic60 displays a conserved membrane-bending activity that is necessary for normal cristae morphology. J Cell Biology 216, 889-899.

Toth, A., Meyrat, A., Stoldt, S., Santiago, R., Wenzel, D., Jakobs, S., Ballmoos, C. von, and Ott, M. (2020). Kinetic coupling of the respiratory chain with ATP synthase, but not proton gradients, drives ATP production in cristae membranes. P Natl Acad Sci Usa 117, 2412-2421.

Vogel, F., Bornhövd, C., Neupert, W., and Reichert, A.S. (2006). Dynamic subcompartmentalization of the mitochondrial inner membrane. J Cell Biology 175, 237-247.

Votruba, M. (2004). Molecular genetic basis of primary inherited optic neuropathies. Eye 18, 1126-1132.

Wallace, D.C. (2005). A Mitochondrial Paradigm of Metabolic and Degenerative Diseases, Aging, and Cancer: A Dawn for Evolutionary Medicine. Annu Rev Genet 39, 359-407.

Wang, C., Taki, M., Sato, Y., Tamura, Y., Yaginuma, H., Okada, Y., and Yamaguchi, S. (2019). A photostable fluorescent marker for the superresolution live imaging of the dynamic structure of the mitochondrial cristae. P Natl Acad Sci Usa 116, 15817-15822.

Weber, T.A., Koob, S., Heide, H., Wittig, I., Head, B., Bliek, A. van der, Brandt, U., Mittelbronn, M., and Reichert, A.S. (2013). APOOL is a cardiolipin-binding constituent of the Mitofilin/MINOS protein complex determining cristae morphology in mammalian mitochondria. Plos One 8, e63683.

Westermann, B. (2010). Mitochondrial fusion and fission in cell life and death. Nat Rev Mol Cell Bio 11, 872-884.

Wiedemann, N., and Pfanner, N. (2017). Mitochondrial Machineries for Protein Import and Assembly. Annu Rev Biochem 86, 685-714.

Wiedemann, N., Kozjak, V., Chacinska, A., Schönfisch, B., Rospert, S., Ryan, M.T., Pfanner, N., and Meisinger, C. (2003). Machinery for protein sorting and assembly in the mitochondrial outer membrane. Nature 424, 565-571.

Wolf, D.M., Segawa, M., Kondadi, A.K., Anand, R., Bailey, S.T., Reichert, A.S., Bliek, A.M., Shackelford, D.B., Liesa, M., and Shirihai, O.S. (2019). Individual cristae within the same mitochondrion display different membrane potentials and are functionally independent. Embo J 38, e101056.

Wong, E.D., Wagner, J.A., Gorsich, S.W., McCaffery, J.M., Shaw, J.M., and Nunnari, J. (2000). The Dynamin-Related Gtpase, Mgm1p, Is an Intermembrane Space Protein Required for Maintenance of Fusion Competent Mitochondria. J Cell Biology 151, 341-352.

Wurm, C.A., and Jakobs, S. (2006). Differential protein distributions define two sub-compartments of the mitochondrial inner membrane in yeast. Febs Lett 580, 5628-5634.

Xie, J., Marusich, M.F., Souda, P., Whitelegge, J., and Capaldi, R.A. (2007). The mitochondrial inner membrane protein Mitofilin exists as a complex with SAM50, metaxins 1 and 2, coiled-coil-helix coiledcoil-helix domain-containing protein 3 and 6 and DnaJC11. Febs Lett 581, 3545-3549.

Yamaguchi, R., and Perkins, G. (2009). Dynamics of mitochondrial structure during apoptosis and the enigma of Opa1. Biochim Biophys Acta 1787, 963-972. 
Yamano, K., Tanaka-Yamano, S., and Endo, T. (2010). Mdm10 as a dynamic constituent of the TOB/SAM complex directs coordinated assembly of Tom40. Embo Rep 11, 187-193.

Yan, L., Qi, Y., Ricketson, D., Li, L., Subramanian, K., Zhao, J., Yu, C., Wu, L., Sarsam, R., Wong, M., et al. (2020). Structural analysis of a trimeric assembly of the mitochondrial dynamin-like GTPase Mgm1. P Natl Acad Sci Usa 117, 4061-4070.

Yang, C., and Svitkina, T.M. (2019). Ultrastructure and dynamics of the actin-myosin II cytoskeleton during mitochondrial fission. Nat Cell Biol 21, 603-613.

Youle, R.J., and Bliek, A.M. van der (2012). Mitochondrial fission, fusion, and stress. Sci New York N Y $337,1062-1065$.

Zerbes, R.M., Bohnert, M., Stroud, D.A., Malsburg, K. von der, Kram, A., Oeljeklaus, S., Warscheid, B., Becker, T., Wiedemann, N., Veenhuis, M., et al. (2012). Role of MINOS in Mitochondrial Membrane Architecture: Cristae Morphology and Outer Membrane Interactions Differentially Depend on Mitofilin Domains. J Mol Biol 422, 183-191.

Zerbes, R.M., Höß, P., Pfanner, N., Laan, M. van der, and Bohnert, M. (2016). Distinct Roles of Mic12 and Mic27 in the Mitochondrial Contact Site and Cristae Organizing System. J Mol Biol 428, 1485-1492.

Zhang, Z., Wakabayashi, N., Wakabayashi, J., Tamura, Y., Song, W.-J., Sereda, S., Clerc, P., Polster, B.M., Aja, S.M., Pletnikov, M.V., et al. (2011). The dynamin-related GTPase Opa1 is required for glucose-stimulated ATP production in pancreatic beta cells. Mol Biol Cell 22, 2235-2245.

Zick, M., Rabl, R., and Reichert, A.S. (2008). Cristae formation-linking ultrastructure and function of mitochondria. Biochim Biophys Acta 1793, 5-19.

Zick, M., Duvezin-Caubet, S., Schäfer, A., Vogel, F., Neupert, W., and Reichert, A.S. (2009). Distinct roles of the two isoforms of the dynamin-like GTPase Mgm1 in mitochondrial fusion. Febs Lett 583, 2237-2243. 


\section{Appendix}

\section{List of Figures}

Figure 1: Mitochondria form networks. 1

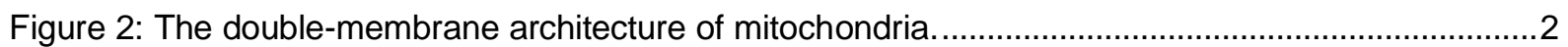

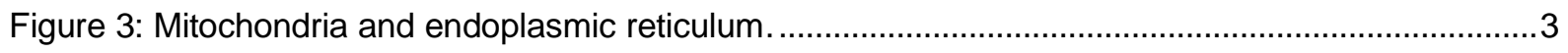

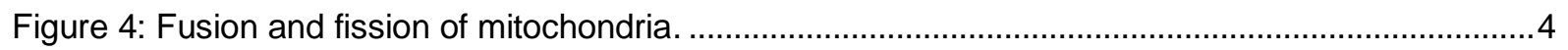

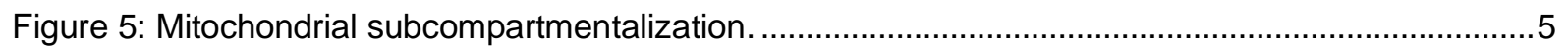

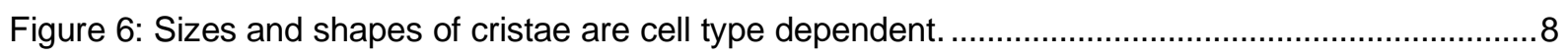

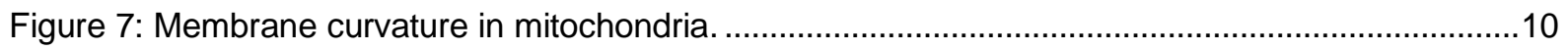

Figure 8: Structure of the dimeric $\mathrm{F}_{1} \mathrm{~F}_{\mathrm{O}}$-ATP synthase from Saccharomyces cerevisiae. ......................11

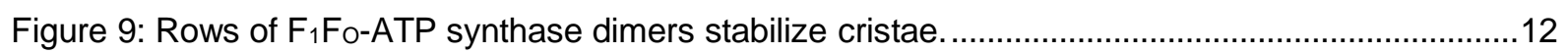

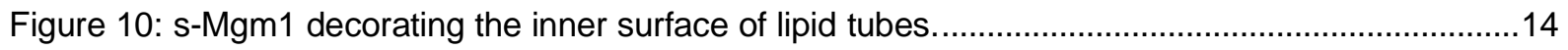

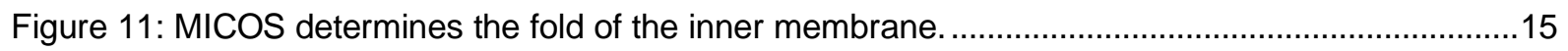

Figure 12: Mic60 shows membrane-bending activity. .....................................................................17

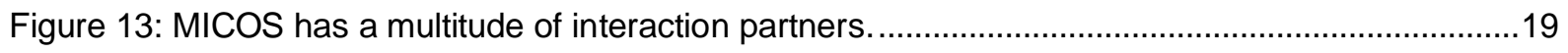

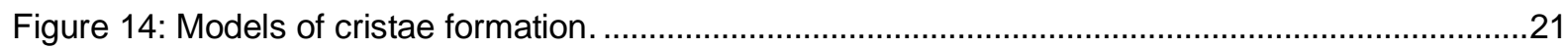

Figure 15: Models of cristae formation following outer membrane fusion. ............................................22

Figure 16: Model of cristae formation in the yeast Saccharomyces cerevisiae......................................24

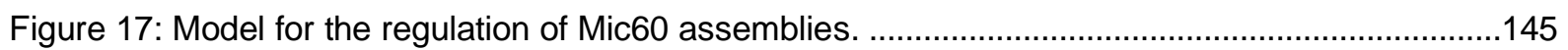

Figure 18: Model for lamellar cristae formation. ......................................................................146

\section{List of Tables}

Table 1: Overview of MICOS subunits.

\section{Abbreviations}

$\begin{array}{ll}\Delta & \text { Deletion (knockout) } \\ { }^{\circ} \mathrm{C} & \text { Degree Celsius } \\ \mu & \text { Micro } \\ 2 \mathrm{D} & \text { Two dimensional } \\ 3 \mathrm{D} & \text { Three dimensional } \\ \mathrm{A} & \text { Ampere } \\ \text { AAVS1 } & \text { Adeno-associated virus integration site 1 } \\ \text { ADP } & \text { Adenosine diphosphate } \\ \text { AMP } & \text { Adenosine monophosphate } \\ \text { ATP } & \text { Adenosine triphosphate } \\ \text { ATP5B } & \text { ATP synthase subunit beta } \\ \text { ATP5ME } & \text { ATP synthase membrane subunit E } \\ \text { ATPb } & \text { ATP synthase subunit beta } \\ \text { BAX } & \text { Bcl2-associated } X \text { protein }\end{array}$


Appendix

bp

BSA

C-

CAG-

Cas9

cGAS

CJ

COX

COX8A

CRISPR

d

DNA

DNAJC11

Dnm1

DRP1

EGTA

EM

ERMES

ET

$\mathrm{FADH} 2$

FIB-SEM

FLAG

Fzo1

$\mathrm{g}$

GFP

GMP

GTP

h

HDFa

HeLa

HEPES

IBM

IM

IMS

k

KD

$\mathrm{kDa}$

$\mathrm{KO}$

kV

I

I-

LETM1

LUV

$\mathrm{m}$

$\mathrm{mA}$

$\mathrm{M}$
Base pairs

Bovine serum albumin

Carboxy-

Promotor combining CMV early enhancer element, beta-chicken actin promotor and splice acceptor of rabbit beta-globin gene

CRISPR-associated protein 9

Cyclic GMP-AMP synthase

Crista junction

Cytochrome c oxidase

Cytochrome $\mathrm{c}$ oxidase subunit $8 \mathrm{~A}$

Clustered regularly interspaced short palindromic repeats

Day

Deoxyribonucleic acid

DnaJ Heat Shock Protein Family (Hsp40) Member C11

Dynamin-related protein 1

Dynamin1-like protein, dynamin-related protein 1

Ethylene glycol-bis ( $\beta$-aminoethyl ether)- $\mathrm{N}, \mathrm{N}, \mathrm{N}^{\prime}, \mathrm{N}^{\prime}$-tetraacetic acid

Electron microscopy

Endoplasmic reticulum -mitochondria encounter structure

Electron tomography

Flavin adenine dinucleotide

Focused ion beam scanning electron microscopy

Polypeptide of the amino acids DYKDDDDK

Fuzzy onions homolog 1

Gramm

Green fluorescent protein

Guanosine monophosphate

Guanosine triphosphate

Hour

Adult human dermal fibroblast

Henrietta Lacks

2-[4-(2-hydroxyethyl)piperazin-1-yl]ethanesulfonic acid

Inner boundary membrane

Inner membrane

Intermembrane space

Kilo

Knockdown

Kilo Dalton

Knockout

Kilovolt

Liter

Long (Isoform)

Leucine zipper-EF-hand containing transmembrane protein 1

Large unilamellar vesicles

Meter or milli

Milliampere

Molar 


\begin{tabular}{|c|c|}
\hline MFN1 & Mitofusin 1 \\
\hline MFN2 & Mitofusin 2 \\
\hline Mgm1 & Mitochondrial genome maintenance 1 \\
\hline MIA & Mitochondrial import and assembly machinery \\
\hline MIB & Mitochondrial intermembrane space bridging \\
\hline MICOS & Mitochondrial contact site and cristae organizing system \\
\hline $\min$ & Minute \\
\hline MINFLUX & Minimal photon fluxes \\
\hline MINOS & Mitochondrial inner membrane organizing system \\
\hline MIRO & Mitochondrial Rho \\
\hline MitOS & Mitochondrial organizing structure \\
\hline $\mathrm{ml}$ & Milliliter \\
\hline mtDNA & Mitochondrial DNA \\
\hline MTX1/2 & Metaxin-1/2 \\
\hline $\mathrm{n}$ & Nano \\
\hline $\mathrm{NADH}$ & Nicotinamide adenine dinucleotide \\
\hline NDUFA10 & $\mathrm{NADH}$ dehydrogenase [ubiquinone] 1 alpha subcomplex subunit 10 \\
\hline $\mathrm{nm}$ & Nanometer \\
\hline OM & Outer membrane \\
\hline OPA1 & Optic atrophy 1 \\
\hline OXPHOS & Oxidative Phosphorylation \\
\hline $\mathrm{p}$ & Pico \\
\hline PAGE & Polyacrylamide gel electrophoresis \\
\hline PALM & Photoactivated localization microscopy \\
\hline PBS & Phosphate buffered saline \\
\hline PCR & Polymerase chain reaction \\
\hline PHEM & PIPES-HEPES-EGTA-Magnesium \\
\hline PIPES & Piperazine-N,N'-bis(2-ethanesulfonic acid) \\
\hline Por1 & Porin 1 \\
\hline RESOLFT & Reversible saturable optical fluorescence transitions \\
\hline RNA & Ribonucleic acid \\
\hline RNAi & RNA interference \\
\hline $\mathrm{RT}$ & Room temperature \\
\hline s- & short (Isoform) \\
\hline SAM & Sorting and assembly machinery \\
\hline SDHA & Succinate dehydrogenase complex subunit $A$ \\
\hline sec & Second \\
\hline SIM & Structured illumination microscopy \\
\hline siRNA & Silencing RNA \\
\hline STED & Stimulated emission depletion \\
\hline STING & Stimulator of interferon genes \\
\hline STORM & Stochastic optical reconstruction microscopy \\
\hline TCA & Tricarboxylic acid \\
\hline TEM & Transmission electron microscopy \\
\hline TetOn & Tetracycline-On \\
\hline TEV & Tobacco Etch Virus \\
\hline
\end{tabular}


Appendix

TIM

$-\mathrm{TO}$

TOM

TRAK

tRNA

Ugo1

$\mathrm{V}$

VDAC

$\times g$
Translocase of the inner membrane

Tetracycline-On

Translocase of the outer membrane

Trafficking kinesin protein

Transfer RNA

Fusion 1

Volt

Voltage-dependent anion channel

Multiple of the gravitational constant 


\section{Acknowledgements}

In the first place, I would like to thank my supervisor Prof. Dr. Stefan Jakobs for giving me the opportunity to study exciting aspects of mitochondrial biology in a remarkable scientific environment. He has been an encouraging and supporting mentor and I am grateful for his confidence, his deep interest in the project and the frequent discussions that helped to overcome many of the challenges during the project.

I am grateful to my colleagues, who constantly supported me in successfully completing this project. I especially thank Dr. Stefan Stoldt and Dr. Christian Brüser for sharing their expertise in STED nanoscopy and for our collaborative work on the MICOS complex. I am grateful to Dr. Mariam Barbot, who shared her extensive experience with MICOS and her expertise in protein biochemistry whenever possible. I would like to thank particularly Dr. Dietmar Riedel, Gudrun Heim and Felix Lange for their enormous efforts in recording electron micrographs of mitochondria. I am also grateful to Dr. Peter Ilgen for his efforts to create some of the beautiful 3D animations that allowed visualizing the FIB-SEM recordings presented in this thesis. Furthermore, I thank Axel Rösch for the fruitful collaboration on live-cell STED nanoscopy and Rita Schmitz-Salue for her excellent technical assistance. I would also like to thank Dr. Stefan Stoldt, Dr. Isabelle Jansen, Dr. Mariam Barbot and Tiberiu Mihaila for proofreading of the thesis.

I am grateful to all past and present members of the department as they created an inspiring and nice working atmosphere during the time in the lab. I express my sincere thanks to the members of my office, Dr. Isabelle Jansen, Dr. Timo Konen, Dr. Peter Ilgen and Dr. Carola Gregor for countless conversations but also entertainment and encouragement in challenging situations. You and all other members of the group made this time in the laboratory memorable.

I would like to thank our collaboration partners Prof. Dr. Peter Rehling, Dr. Markus Deckers, Dr. Wiebke Möbius and Dr. Anna Steyer who contributed to the success of the multidisciplinary MICOS project. I am also grateful to Prof. Dr. Stefan W. Hell, Dr. Jasmin Pape and Dr. Francisco Balzarotti for the unique opportunity to analyze my cells using the novel MINFLUX nanoscopy and I thank Jan Keller-Findeisen for his recurring help with data analysis.

Moreover, I would like to thank Prof. Dr. Ralf Ficner for his support as a member of my thesis committee and Prof. Dr. Stefan W. Hell, Prof. Dr. Peter Rehling, Prof. Dr. Michael Thumm and PD Dr. Thomas Teichmann for being part of the extended examination board.

Finally, I want to express my deepest gratitude towards my parents, my brother and my grandmother for their unconditional support. 